\title{
LBL 3253
}

\section{Energy and} Environment Annual Report 1974

Energy and Environment Division

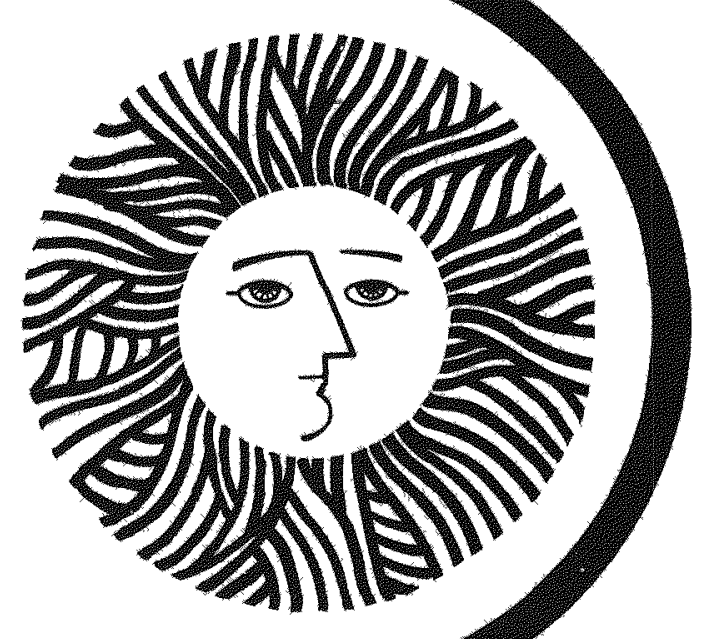

Lawrence Berkeley Laboratory

University of California 


\section{DISCLAIMER}

This report was prepared as an account of work sponsored by an agency of the United States Government. Neither the United States Government nor any agency Thereof, nor any of their employees, makes any warranty, express or implied, or assumes any legal liability or responsibility for the accuracy, completeness, or usefulness of any information, apparatus, product, or process disclosed, or represents that its use would not infringe privately owned rights. Reference herein to any specific commercial product, process, or service by trade name, trademark, manufacturer, or otherwise does not necessarily constitute or imply its endorsement, recommendation, or favoring by the United States Government or any agency thereof. The views and opinions of authors expressed herein do not necessarily state or reflect those of the United States Government or any agency thereof. 


\section{DISCLAIMER}

Portions of this document may be illegible in electronic image products. Images are produced from the best available original document. 


\section{ENERGY AND ENVIRONMENT ANNUAL REPORT 1974}

Jack M. Hollander

Division Head

Carl Blumstein

Editor

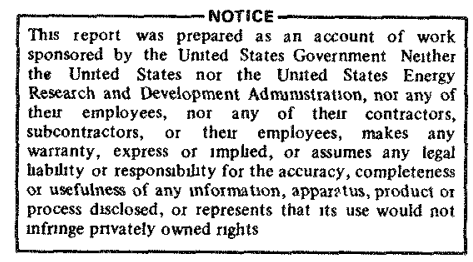

Work done under U. S. Energy Research and Development Administration Contract No. W-7405-ENG-48

Energy and Environment Division

Lawrence Berkeley Laboratory

University of California

Berkeley, California 94720 
$-i i-$

\section{LEGAL NOTICE}

This report was prepared as an account of work sponsored by the United States Government. Neither the United States nor the United States Energy Research and Development Administration, nor any of their employees, nor any of their contractors, subcontractors, or their employees, makes any warranty, express or implied, or assumes any legal liability or responsibility for the accuracy, completeness or usefulness of any information, apparatus, product or process disclosed, or represents that its use would not infringe privately owned rights.

Printed in the United States of America Available from

National Technical Information Service

U.S. Department of Commerce

5285 Port Royal Road

Springfield, Virginia 22161

Price: Printed Copy $\$ 7.00$; Microfiche $\$ 2.25$ 
Introduction . . . . . . . . . . . . . . . . . . . . . . . . . . . . . 1

Stability Properties and Dynamics of Ecosystems John Harte and Don Levy . . . . . . . . . . . . . . . . . . . . . . . . . . . 1

Formation of Pollution Particulate Nitrogen Compounds by NO-Soot and $\mathrm{NH}_{3}$-Soot gas Particle Surface Reactions S. G. Chang and T. Novakov. . . . . . . . . . . . . . . . . . . . . . . .

Determination of the Chemical States of Sulfur in Ambient Pollution Aerosols by

X-ray Photoelectron Spectroscopy

N. L. Craig, A. B. Harker and T. Novakov. . . . . . . . . . . . . . . . . . . . 8

Sulfates as Pollution Particulates: Catalytic Formation on Carbon (Soot) Particles T. Novakov, S. G. Chang and A. B. Harker. . . . . . . . . . . . . . . . . . . 9

Distribution of Trace Element Aerosols in Urban Air W. Winklestein, Jr., S. Sacks, and R. D. Giauque. . . . . . . . . . . . . . . . . . 11

Cadmium Levels in the Shoreline Sediments of San Francisco Bay B. R. Moyer and T. F. Budinger. . . . . . . . . . . . . . . . . . . . . . . . . 13

Partitioning of Lead in Estuarine Waters of San Francisco Bay Don Girvin. . . . . . . . . . . . . . . . . . . . . . . . . . . . . . 15

Dredging Impact-Heavy Metals Uptake Study Victor C. Anderlini, John W. Chapman, Donald G. Girvin, Amos S. Newton, and Robert W. Risebrough. . . . . . . . . . . . . . . . . . . . . . . . . .

Effect of Pollutants on Membrane Structure and Function in Mammalian Ce11s L. Packer, R. Mehthorn, and J. R. Smith........................

\section{SOLAR ENERGY RESEARCH AND DEVELOPMENT}

Introduction . . . . . . . . . . . . . . . . . . . . . . . . . . . . . . .

Solar Cel1 Research

Wigbert J. Siekhaus, Gabor A. Somorjai, Chin-An Chang, Jim Cmmp, Donald

Tai-Chan Huo, and Tom Kaminska. . . . . . . . . . . . . . . . . . . . . . . . . . .

Chloroplast Membrane Structure and Photosynthetic Electron Transport Stability

G. Papageorgiou, G. D. Case, S. Hansen, and L. Packer . . . . . . . . . . . . . .

Solar Heating and Cooling of Buildings

M. Wahlig, E. Binnall, R. Graven, F. Selph, R. Show, M. Simmons, J. Tanabe, and $R$. Wolgast. ................................

Nitinol Engine Project

R. Banks, J. Gunn, P. Hernandez, D. Johnson, H. Mohamed, R. Nickerson,

D. Norgren, J. Washburn, O. Weres . . . . . . . . . . . . . . . . . . . .

Studies of Cellulose as a Chemical and Energy Resource

Charles R. Wilke, Paul Carroad, Gerald R. Cysewski, Malcolm Evett, Raymond Freitas, Gautam Mitra, Beverly Rubik, Albert Sciamenna, Yoshio Yamanaka,

and R. D. Yang. . . . . . . . . . . . . . . . . . . . . . . . . . . .

Measurement of Circumsolar Radiation

D. E. Grether, J. E. Nelson, and M. Wahtig. . . . . . . . . . . . . . . . .

INSTRUMENTATION AND MEASUREMENT

Introduction . . . . . . . . . . . . . . . . . . . . . . . . . . . . 44

The Survey of Instrumentation for Environmental Monitoring

D. A. Mack, N. M. Amer, R. J. Budnitz, R. M. Graven, C. C. Hollowell, M. J. KZand, R. A. MeLaughiz, W. D. Macteod, G. A. Morton, S. L. Phizlips, and J. A. Stokes. . . . . . . . . . . . . . . . . . . . . . . . . 44

Detection and Measurements of Air Pollutants by Microwave Rotational Spectroscopy

B. Leskovar, W. F. Kolbe, and D. B. Hopkins........................ 45

gh Purity Germanium

WiZZiam L. Hansen and Eugene E. HaZler. . . . . . . . . . . . . . . . . . . . . . . 47

Impurities in High-Purity Germanium as Determined by Fourier Transform Spectroscopy

William L. Hansen and Eugene E. Haller. . . . . . . . . . . . . . . . . . . 50 
Application of a Low Energy X-ray Spectrometer to Analysis of Suspended Air

Particulate Matter

R. D. Giauque, R. B. Garrett, I. Y. Goda, J. M. Jaklevic, and D. F. Matone. . . . . . . . 54

The Remote Detection of Pollutants Using Resonance Raman Scattering

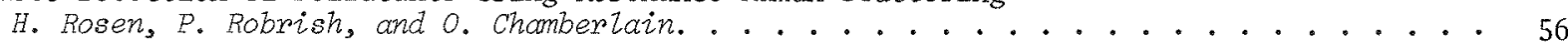

Cholesteric Liquid Crystal as a Sensitive Gas Detector

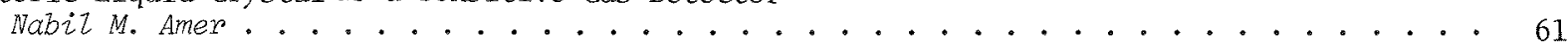

Direct Flameless Atomic Absorption Analysis of Samples Weighing Several Tenths of

a Gram

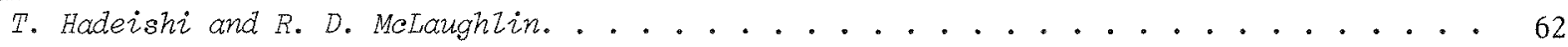

An Improved Technique for the Quantitative Assay of Soils and Sediments for

Cadmium

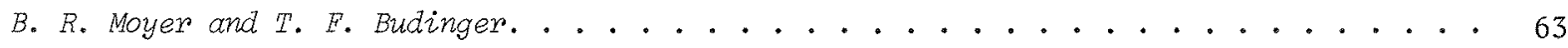

Cause of Loss of Hydrogen Overvoltage at Graphite Electrodes Used for Anodic

Stripping Voltametry

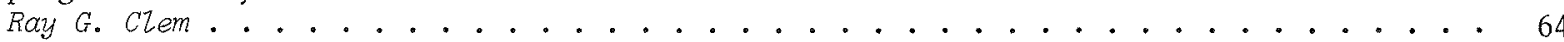

Aqueous Sulfide Level Determinations to Soft X-ray Fluorescence Analysis

A. J. Hebert. . . . . . . . . . . . . . . . . . 65

Point Measurement of $\mathrm{NO}_{2}$

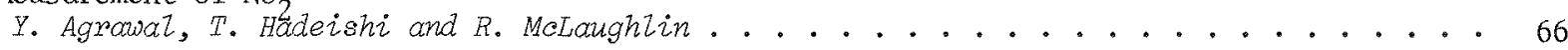

NEW TECHNOLOGY AND SPIN-OFF RESEARCH

Introduction . . . . . . . . . . . . . . . . . . . . . . . 67

Intense Sub-microsecond Electron Bursts Produce Rock Shattering

Robert T. Avery, Denis Keefe, Tor L. Brekke, and Iain Finnie. . . . . . . . . . . 67

Studies Concerning Rapid Transit

D. Theodore Scalise, Don M. Evans, Allan A. Arthur, David Bereznai, Martin

Graham, Raymond Louis, Kenneth G. Wiley................. 69

Prevention of Sickling of Human Erythrocytes in Imidoesters

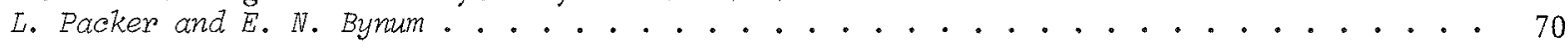

Luminescence of CdS Generated by High Intensity Excitation Below the Band Gap

A. Mysyrowicz, A.J. Schmidt, Y. R. Shen, P. Robrish, and H. Rosen. . . . . . . . 72

FOSSIL ENERGY RESEARCH AND DEVELOPMENT

Introduction . . . . . . . . . . . . . . . . . . . . . . 75

Evaluation of Catalysts for Coal Gasification

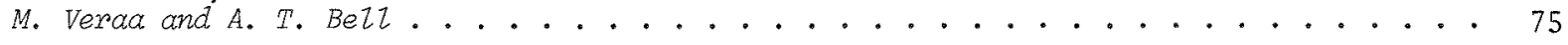

Coal Conversion Catalysis

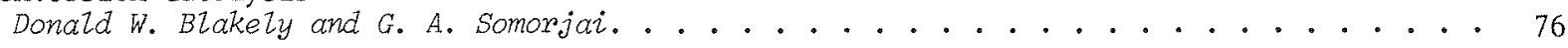

Selective Hydrogenation of Coal: Chemical Engineering Aspects

E. A. Grens, E. E. Petersen, A. T. Bell, T. Vermeulen, K. ConkIin,

T. Derencesenyi, D. DraemeZ, L. Jossens, D. J. Medeiros, and S. Salim. . . . . . . . 76

Synthesis of Hydrocarbons from $\mathrm{CO}-\mathrm{H}_{2}$ Mixtures Produced By Coal Gasification

W. Bollinger, J. Ekerdt, and A. T. Bell .................... 79

Reduction and Removal of $\mathrm{SO}_{2}$ and $\mathrm{NO}_{\mathrm{x}}$ from Simulated Flue Gas With Use of Iron

Oxide and Catalysis/Absorbent

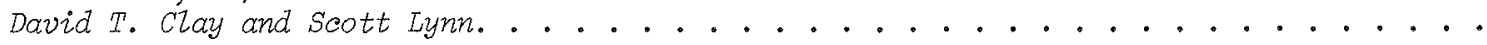

GEOTHERMAL ENERGY AND THE GEOSCIENCES

Introduction . . . . . . . . . . . . . . . . . . . . . . . . 81

Geothermal Energy Resource Assessment

H. A. Wollenberg, F. Asaro, H. Bouman, T. MeEvilly, F. Morrison, and P.

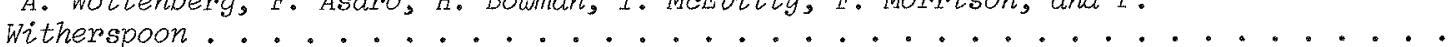

Experimental Power Generation Facility

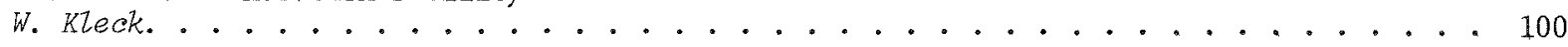


Thermodynamic Process Program for Geothermal Power Plant Cycles

M. A. Green, A. D. K. Laird, and H. S. Pines. . . . . . . . . . . . . . . . . . . . . . 102

Predicting the Temperature at Depth of Natural Hot and Cold Springs in Geothermal.

We11 Waters

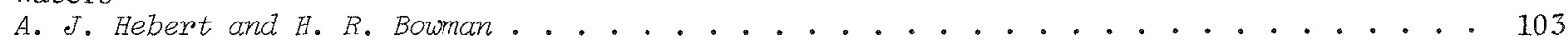

Thermodynamic Properties of High Temperature Brines

K. S. Pitzer and L. F. Silvester. . . . . . . . . . . . . . . . . . . . 104

Background Studies of Predicting Silicic Acid Behavior in Geothermal Brines

A. R. Marsh, I. Huang, G. Klein, and T. VermeuZen . . . . . . . . . . . . . . . . 105

Measurement of the Properties and Behavior of Rock-Fluid Systems at Elevated

Pressures and Temperatures

W. H. Somerton. . . . . . . . . . . . . . . . . . . . . 107

Geotherma1 Reservoir Dynamics

P. A. Witherspoon and V. E. Schrock.......................... 108

National Geothermal Information Resource (GRID)

F. B. Henderson III, S. L. Philizips, and T. Trippe. . . . . . . . . . . . . . 109

PLASMA PHYSICS AND CONTROLLED FUSION RESEARCH (CTR)

Introduction . . . . . . . . . . . . . . . . . . . . . . . . . . . . . . . . 11

Plasma Production and Trapping of Laser-Ionized Pure Deuterium Pellet Plasma in a

Magnetic Field

N. E. Abt, T. R. Jarboe, W. B. Kunkel, A. F. Liezke, and G. H. Rankin........... 112

Atomic Physics Studies

K. H. Berkner, W. G. Graham, D. Leung, T. J. Morgan, R. V. Pyze, S. E. Savas,

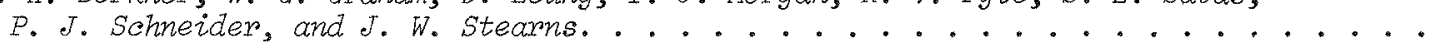

Neutral Beam Development and Technology

W. R. Baker, K. H. Berkner, W. S. Cooper, K. W. Ehlers, W. B. KunkeZ,

R. V. Pyle, and J. W. Stearns...........................116

Tormac Sheath Confinement

M. A. Levine, I. G. Brown, and W. B. Kunkel . . . . . . . . . . . . . . . . . . . 117

Parametric Instabilities in Turbulent, Inhomogeneous Plasma

D. R. Nicholson and A. N. Koufman . . . . . . . . . . . . . . . . . . . . . . 119

Action Conservation in the Presence of a High Frequency Field

A. N. Kaufman and L. Stenflo. . . . . . . . . . . . . . . . . . . . . . . . . . 119

Nonlinear Penetration of a Langmuir Wave into a Plasma Density Gradient

A. N. Kaufman . . . . . . . . . . . . . . . . . . . . . . . . . . . . 119

Upper-Hybrid Solutions

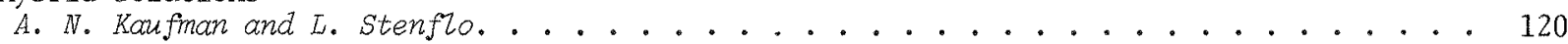

Simulation of Laser Beat Heating of a P1asma

B. Cehen, M. Mostrom, D. Nicholson, C. Max, A. Kaufman, and A. B. Langdon . . . . . . . 120

Simulated Sidescatter in an Inhomogeneous Plasma

Michael A. Mostrom. ..............................

Oscillation-Center Approach to the Classical Theory of Induced Scattering in

Plasma

Shayne Johnston . . . . . . . . . . . . . . . . . . . . . . . . . 


\title{
Environmental Science
}

\author{
INTRODUCTION
}

Research in the Division's environmental science program addresses a broad range of environmental problems. These include air pollution, water pollution, and the effects of pollutants on man and natural ecosystems.

In recent years the Laboratory has played a leading role in the science of airborne particulates (aeroso1s). This work has focused on the chemistry and physics of particle surfaces. Using the technique of electron spectroscopy for chemical analysis (ESCA), surface reactions of sulfur and nitrogen compounds have been studied, and results include the identification of new chemical forms of nitrogen on particle surfaces and evidence for the importance of particle surfaces in the catalysis of sulfur dioxide to sulfuric acid.

The Division's work in water pollution has been devoted to the study of trace metals in the estuarine environment, especially in San Francisco
Bay. Studies on the effect of dredging operations on trace metals in the Mare Island ship channe1 and on the distribution of cadmium in Bay sediments have been performed. Research has also been conducted on the distribution of trace elements between bound states on suspended particles and in solution in Bay waters.

Research is being conducted on a variety of problems relating to effects of pollutants. Biological studies seeking to discover effects of specific environmental insults such as oxidants at the cellular level have been done, and epidemiological studies have been initiated on the impacts of trace metals on human health. Theoretical studies in an attempt to develop a basis for assessing the stability of ecological systems are also being undertaken. The ultimate aim of all of our work in progress is the avoidance of or mitigation of adverse impacts that result from man's activities.

\section{STABILITY PROPERTIES AND DYNAMICS OF ECOSYSTEMS *}

John Harte and Don Levy

\section{INTRODUCTION}

Many technological and social changes can be envisioned during the next decade as a result of changing energy use and modes of production. An understanding of how various ecosytems will respond to perturbations induced by these changes is a necessary ingredient for rational planning and the enhancement of our quality of 1ife. Our work is intended to develop this understanding. In particular, we are concerned with the problem of ecosystem stability.

Our goals in this work are twofold: first, to determine under what perturbations an ecosystem is most unstable (i.e., what kinds of perturbations can most dramatically disrupt ecosystems) and, second, to determine what combinations of ecosystem parameters are reliable indicators of ecosystem stability. Carrying out this program involves theoretical work followed by experimental laboratory and field work with feedback continuing between theory and experiment. Our initial work bas been theoretical in nature; the experimental prk will begin in 1975 . Our work, while focusing n stability properties, also includes studies of other dynamical aspects of ecosystems such as succession, stochasticity, and limit cycle behavior which are needed to understand the fate of an ecosystem.
DISCOVERY OF ECOSYSTEM STABILITY INDICATORS USING THE LIAPUNOV DIRECT METHOD

Currently, much empirical activity in ecology is focused on the measurement of quantities such as the biomasses and productivities of the components of an ecosystem, species diversity, and retention times of various nutrients. These measurements, unfortunately, do not appear to reflect the organization of ecosystems and have not allowed ecologists to infer the stability properties of perturbed ecosystems. 1 We have been able to apply the Liapunov method $^{2}$ of stability analysis to a variety of ecosystem models in standard use today. 3 This has allowed us to characterize certain types of perturbations to which ecosystems are particularly vulnerable and to determine which combinations of the intrinsic system parameters are most indicative of potential instability.

We have considered three broad categories of ecological organization:

1. Open flow without cyling (e.g., flow of energy through the pathways of a food web).

2. Closed flow with cycling (e.g., the global carbon cycle).

3. Open flow with cycling (e.g., the nitrogen flow in a lake or a field).

We have shown that a general form for a Liapunov function 4 for a wide variety of specific models 
describing these systems is

$$
L=\Sigma\left(x_{i}-\bar{x}_{i}-\bar{x}_{i} \ln \frac{x_{i}}{\bar{x}_{i}}\right) c_{i}(\alpha, \beta, \cdots)
$$

where the $\bar{x}_{i}\left(x_{j}\right)$ are the unperturbed (perturbed) values of the system's variables and the $C_{i}$ are complicated but calculable functions of the system's parameters. Utilizing this Liapunov stability function, we have shown that damage to the organic nutrient pool in an ecosystem is a potential source of instability - greater than that arising from perturbations upon the more "visible" predatorprey components of an ecosystem.

In addition, for all the models we have considered, we have found that a particular combination of the parameters describing the flow rates in an ecosystem is highly correlated with stability as well as an indicator of the size of the domain of asymptotic stability of an ecosystem. In the nonlinear dynamical equations describing the flow rates and rates of change of the various compartments in the system, there are two terms describing the loss rate, or decrease, of the active decomposers responsible for nutrient cycling. One is linear in the decomposer mass and describes directly the classical death rate. The other is more complicated and describes the effect of resource scarcity (holding capacity) on decomposer dynamics (there are, of course, other terms describing the growth rate of decomposers). Using our analytic Liapunov technique, we have determined that for a wide class of model systems with nutrient cycling, the stability of the system is largely determined by the ratio of these two rates. (If the ratio of the death rate to the resource scarcity loss rate is large, the system is far more unstable against a wide class of specified perturbations than if it is sma11.) Work is now in progress on developing residence time and tracer techniques to allow in situ measurement of this ratio.

One can also characterize an ecosystem with extermal inputs and outputs as a high subsidy or a low subsidy system. If the rate of nutrient input as compared to the net rate of primary productivity is large (sma11), the system is a high (low) subsidy system. Our analytical work shows that a high subsidy system is more stable against a broad spectrum of perturbations than is a low subsidy system.

COMPUTER SIMULATION OF THE EFFECTS OF PERTURBATIONS ON REALISTIC MODEL ECOSYSTEMS

The analytic results on stability properties of ecosystems which we have obtained using the Liapunov method can be tested and explored further by computer simulation. We emphasize that the analytic Liapunov technique and the numerical computer techniques are complimentary. Without the analytic work, use of computer simulation would be unlikely to lead to identification of stability indicators because of the great difficulty of probing the multitude of possibly significant parameters. On the other hand, the computer simulations are needed to probe the finite regions of stability because our techniques for using the
Liapunov method do not guarantee that we have found the largest finite domain of stability. Furthermore, the computer simulations allow us to determil the explicit time evolution of a perturbed ecosystem.

We have taken two detailed and reasonably realistic models describing a grasslands ecosystem and a freshwater system and determined a11 of the rate coefficients in them from available experimental data. The numerical solutions to the perturbed model equations verify the analytic Liapunov results.

The grasslands model used in these simulations described the nitrogen cycle for a simple model ecosystem made up of four compartments: plants such as western wheatgrass, inorganic nutrient such as $\mathrm{NO}_{3}^{-}$, decomposers, and organic litter (see Fig. 1). In Fig. 2, the response of the system to three different kinds of perturbations is depicted. It shows that the system is most sensitive to disturbances in its organic litter pool, in the sense that a given percent change in the organic litter pool is likely to induce a more significant alteration in the future time development of the system than would a similar percent change in other compartments of the system.

In Fig. 3, the response of a system with a large ratio, $K$, of decomposer death rate to resource scarcity loss rate is compared to one with a small value of this ratio. It shows that this ratio is a stability indicator with sma11 ratios indicating stability. It also shows that the analogous ratio, $K_{p}$, defined for plants is not a sensitive stability indicator. In Fig. 4, a high subsidy system is shown to be more stable than a low subsidy system.

Models for the nitrogen cycle in aquatic systems with ordinary as well as nitrogen-fixing blue-green algae have been explored. By taking into account the oxygen, phosphorous, and nitrogen or phosphorous cycles we expect to be able to determine if our stability indicators correctly predict the onset of eutophication. Application of the grasslands model to the problem of estimating the revegetation potential of stripmined land is also in progress.

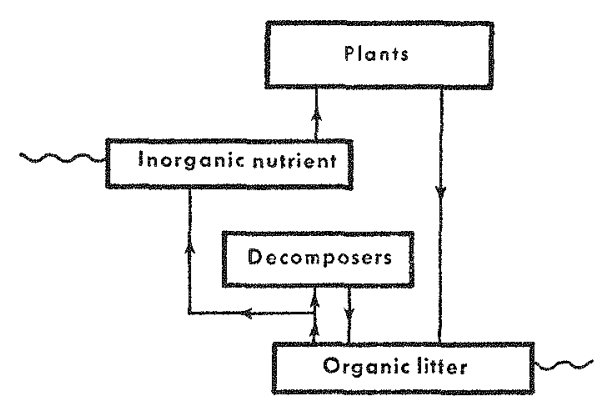

Fig, 1. Nitrogen flow in a simple four-level system. The wiggly lines correspond to external inputs and outputs of nitrogen, and the solid lines denote the flow of nitrogen within the system.
(XBL, 755-2890A) 

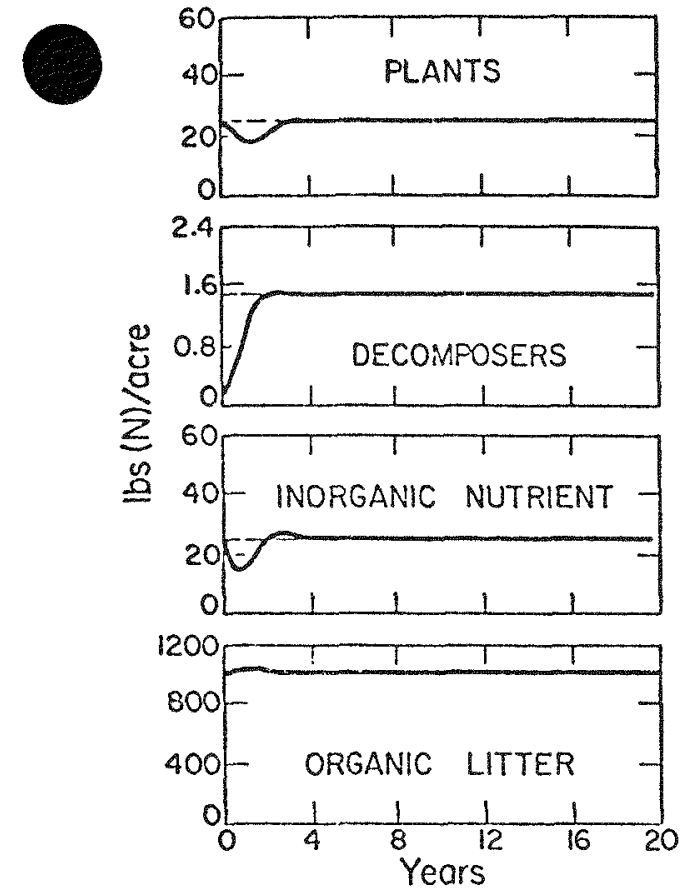

$90 \%$ DECREASE OF DECOMPOSERS
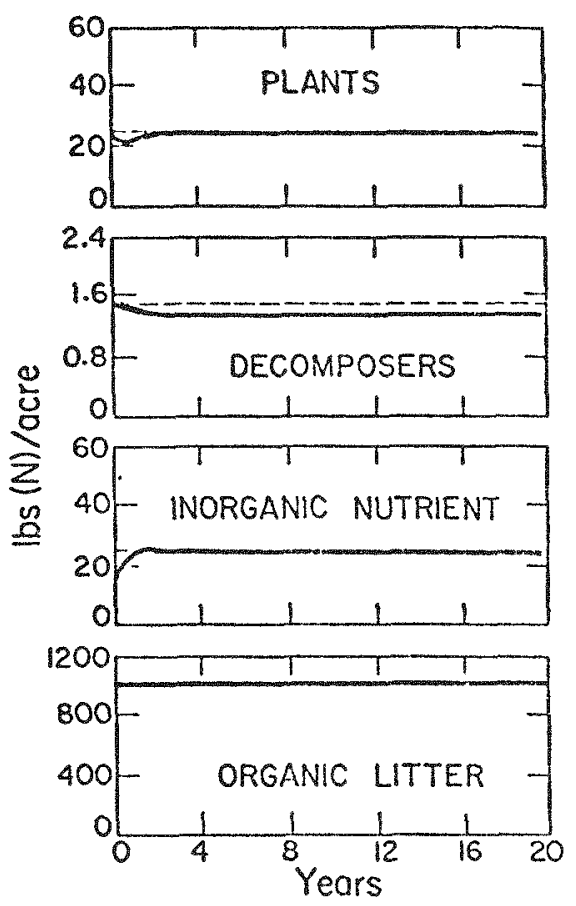

40\% DECREASE OF INORGANIC NUTRIENT
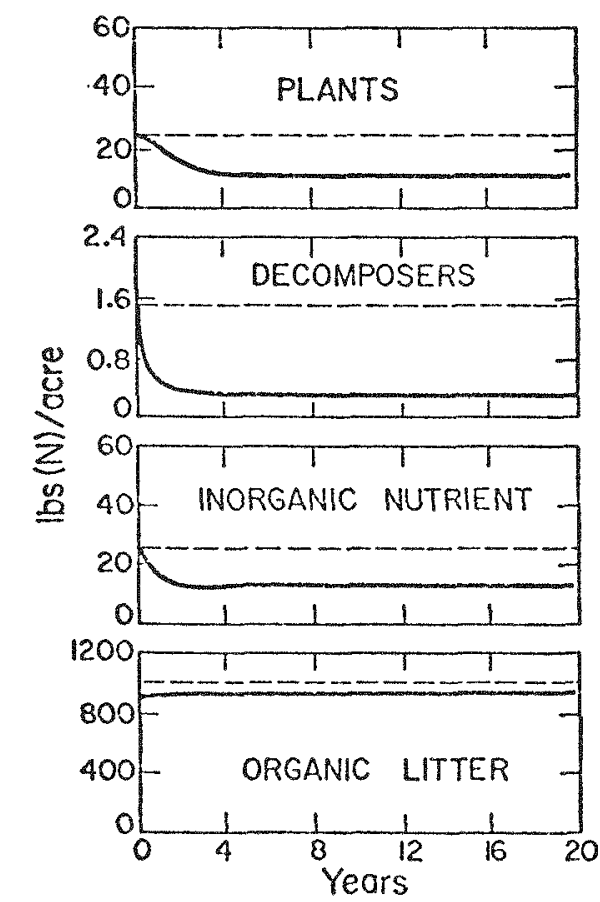

$10 \%$ DECREASE OF ORGANIC LITTER

Fig. 2. Computer simulation of the effect of three different initial disturbances to the four-1evel grassland ecosystem. Note that the litter perturbation has more effect on the system than the other perturbations do.

(XBL 755-2994)
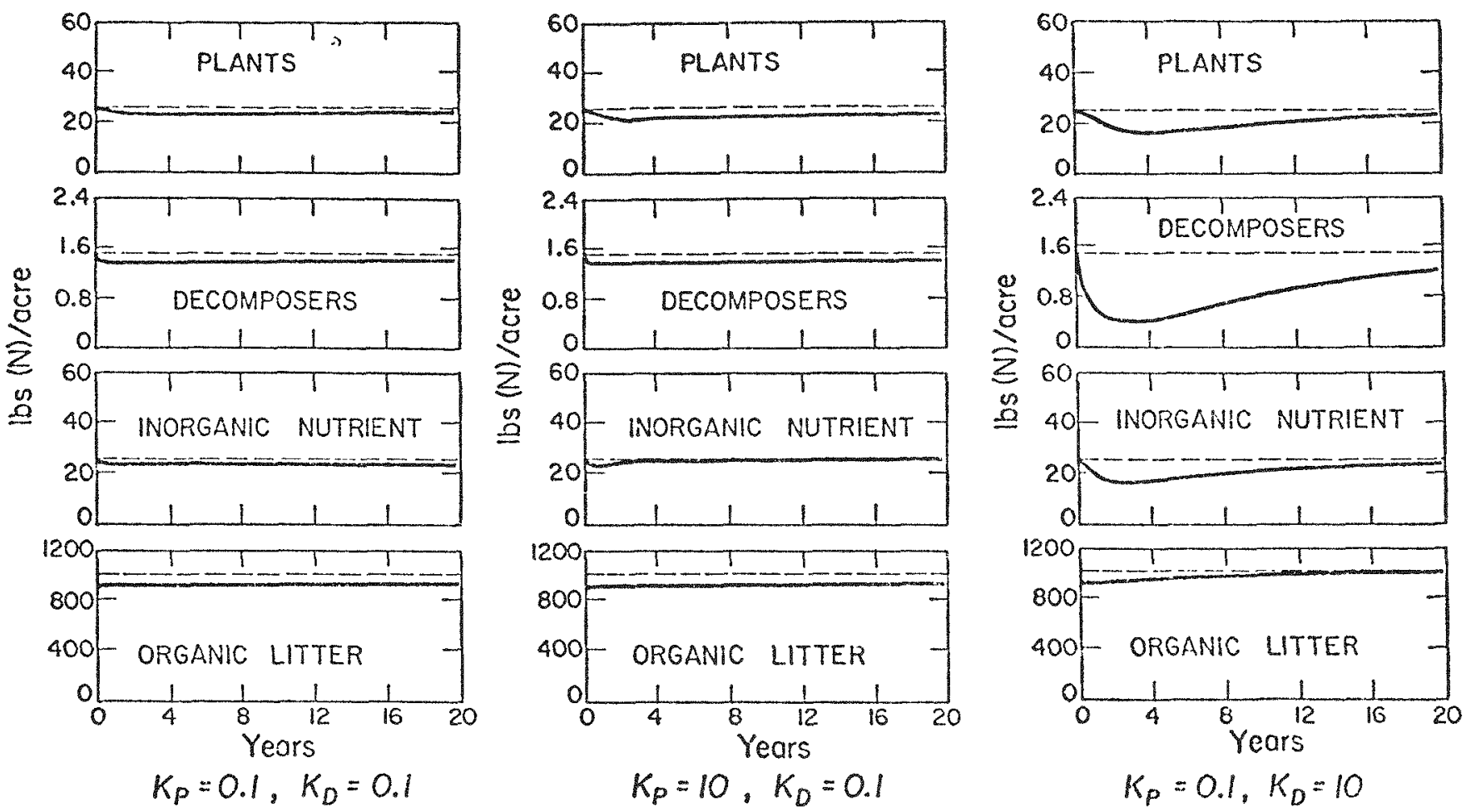

Fig. 3. Computer simulation of the effect of an initial disturbance to the four-1evel grassland ecosystem. The perturbation was chosen to be $10 \%$ reduction in the organic 1itter. Large values of the ratio of decomposer death rate to resource scarcity loss rate ( $\mathrm{K}_{\mathrm{D}}$ ) indicate instability. The analogous ratio defined for plants $\left(K_{p}\right)$ is not a sensitive stability indicator. 

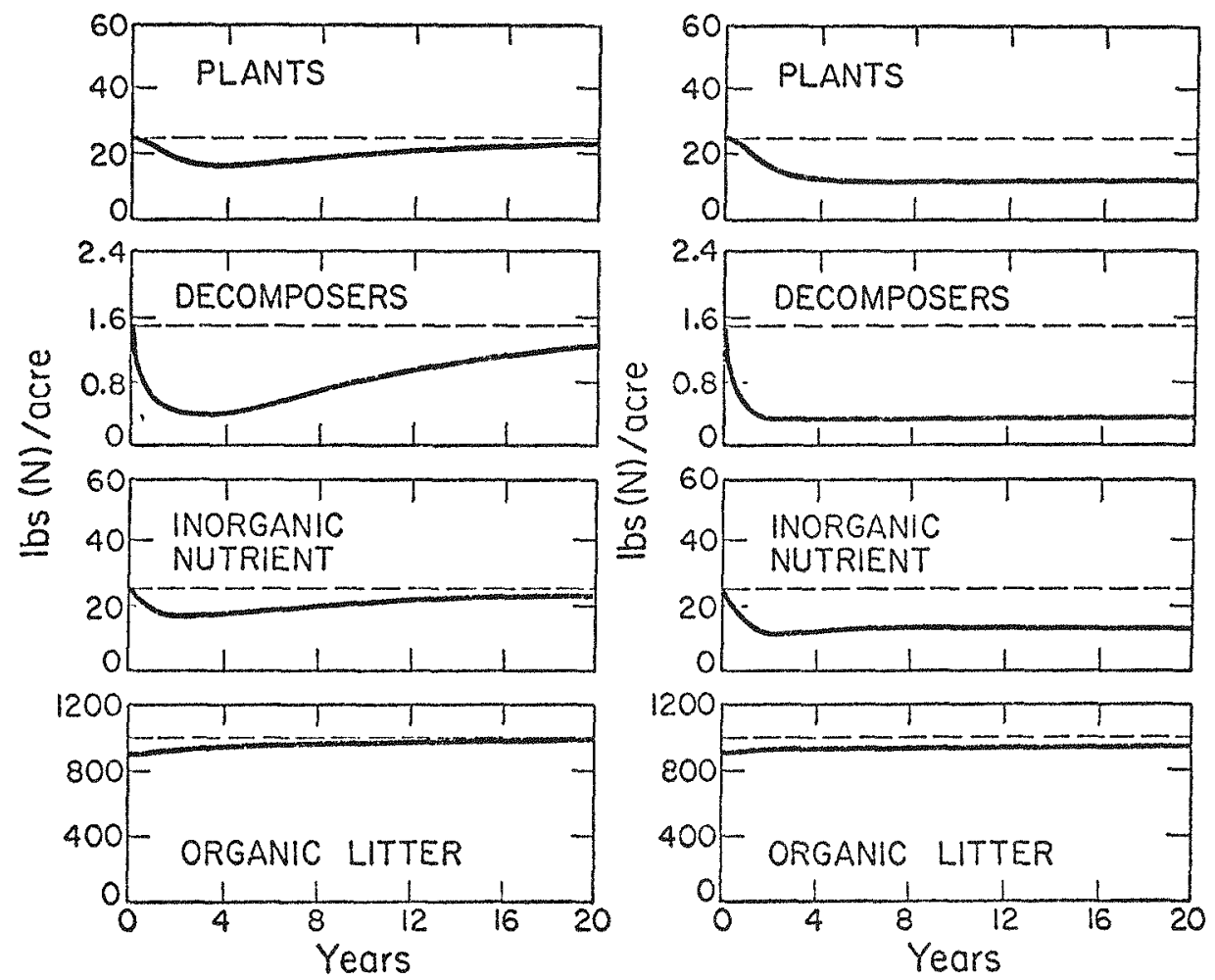

HIGH SUBSIDY

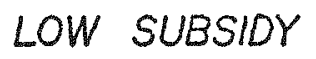

Fig. 4. Computer simulation of the effect of an initial $10 \%$ decrease in organic litter in two systems - one with high subsidy and one with low subsidy. Note that the low subsidy system is more sensitive to perturbations than is the high subsidy system.

(XBL 755-2991)

\section{POSSIBLE ORIGIN OF UNUSUAL CYCLIC PHENOMENA}

A number of natural and social phenomena are a source of curiosity because of their unusual cyclic regularity. Most periodic phenomena with which we are familiar have a relatively straight forward explanation for they are directly linked to the diurnal, or lunar, or annual cycles on earth. Others, however, do not appear to be directly linked to any simple physical cycle. Their periods do not equal a day or a year or any other easily identifiable time interval. One characteristic of these unusual cyclic phenomena is their stability. For example, ecological forces result in population cycles which appear to persist over decades 1 despite changes due to climatic trends and manmade alterations in the natural landscape. Beyond some point, of course, perturbations will disrupt these cycles; in mathematical language the asymptotic stability of these orbits is not global, but rather of finite domain.

An increasing amount of effort is being $\mathrm{d} i$ rected toward a quantitative understanding of such processes. An emerging view is that unusual cyclic phenomena are described by the stable limit-cycle solutions to non-linear differential or difference equations.

We have discovered that an alternate explanation may exist. The classical non linear Lotka-
Volterra (L.V.) equations for interacting populations in a predator-prey type food web with static rate coefficients are known to possess only unstable, oscillatory solutions (if the environmental carrying capacity term in the L.V. model is neglected), or stable, static solutions (if the effect of a finite resource base is included). These are the only non-transient solutions. We have shown by numerical solution, that the L.V. model, with carrying capacity effects included, possess unusual cyclic, and stable, solutions if the rate coefficients in the equations are, themselves, periodic. What makes this result interesting, and unique to non-linear models, is the fact that the generated cyclic solutions can have an associated frequency which is not equal to the frequency of the driving term (as it would be for a linear oscillator) but rather related to the intrinsic frequency of the undriven system. The non-linear equations are rich with structure such as amplitude-dependent sub-hamonics and we are exploring further the possibility that this non-1inear mechanism may explain the existence of certain unusual cyclic phenomena.

Footnotes and References

* Condensed from LBL-3214

1. R. May, "Stability and Complexity in Mode1 Ecosystems". Princeton University press, and references therein (1973). 
2. J. Lasalle, and S. Lefshetz, "Stability by Liapumov's Direct Method". Academic Press (1961).

Included is modeling work at ORNL and ANL (e.g., ORNL EDFB-IBF 74-7, ANL 4935) as well as numerous other ecosystem models described or referred to in reference 1.

4. See H. Huang, and H. Morowitz, Journal of Theoretical Biology, Vo1. 35, p. 389, (1972) for another application of this Liapunov function in ecology.

FORMATION OF POLLUTION PARTICULATE NITROGEN COMPOUNDS

BY NO-SOOT AND $\mathrm{NH}_{3}$-SOOT GAS PARTICLE SURFACE REACTIONS*

\section{S. G. Chang and T. Novakov}

Suspended nitrogen compounds are an important constituent of urban air pollution aerosol particles. Actually, carbon, sulfur and nitrogen compounds, together with water, constitute up to $80 \%$ of the total particle mass. It was commonly assumed that most of the nitrogen compounds in particulate form are in the form of nitrates and ammonium salts, until two additional and unsuspected reduced nitrogen species were identified with the aid of X-ray photoelectron spectroscopy (ESCA). 1 Based on the measured core electron binding energies, these nitrogen species were tentatively assigned to organic compounds of the amino- and pyridino-type. The study of their diurnal concentration variations indicated that these compounds could be primary pollutants coming directly from sources, rather than by way of atmospheric reactions. The nature and origin of these species has remained unknown, however.

We describe here the results we have obtained on more precise chemical characterization of reduced ambient nitrogen species and evidence for their formation via heterogeneous reactions involving nitric oxide and combustion-generated carbonaceous (soot) particles.

Figure 1 illustrates the relative atomic concentrations of various nitrogen species typically associated with ambient air pollution particles. Here ESCA spectra of the nitrogen region of an ambient sample (collected in West Covina, California on August 29, 1973) is reproduced. Peaks corresponding to $\mathrm{NO}_{3}^{-}, \mathrm{NH}_{4}^{+}$and the additional reduced species, $\mathrm{N}_{X}$, are indicated in the figure. The spectra shown were measured in vacuum with the sample at $-150^{\circ} \mathrm{C}$ (Figure 1a) and with the same sample heated up to $25^{\circ} \mathrm{C}$ (Figure 1b). Practically the entire nitrate peak and about 60 percent of the ammonium peak were lost when the sample was warmed up from $-150^{\circ} \mathrm{C}$ to $25^{\circ} \mathrm{C}$. From the $10 \mathrm{w}$ temperature spectrum, it is obvious that at most about $15 \%$ of the ammonium ions could be associated with nitrate as $\mathrm{NH}_{4} \mathrm{NO}_{3}$. Therefore, the remaining ammonium ions must be associated with anions other than nitrate. Because of the limited volitility of common ammonium salts and the observed behavior of ambient ammonium species, we can rule out ammonium bi-sulfate and ammonium sulfate as the major ammonum compounds associated with ambient particles e high volatility of ambient anmonium suggests nat it may be present in some hitherto unsuspected form. The volatility of ambient ammonium is the main reason for the observed systematic inconsistency between ammonium determinations by ESCA and by wet chemical techniques. 2

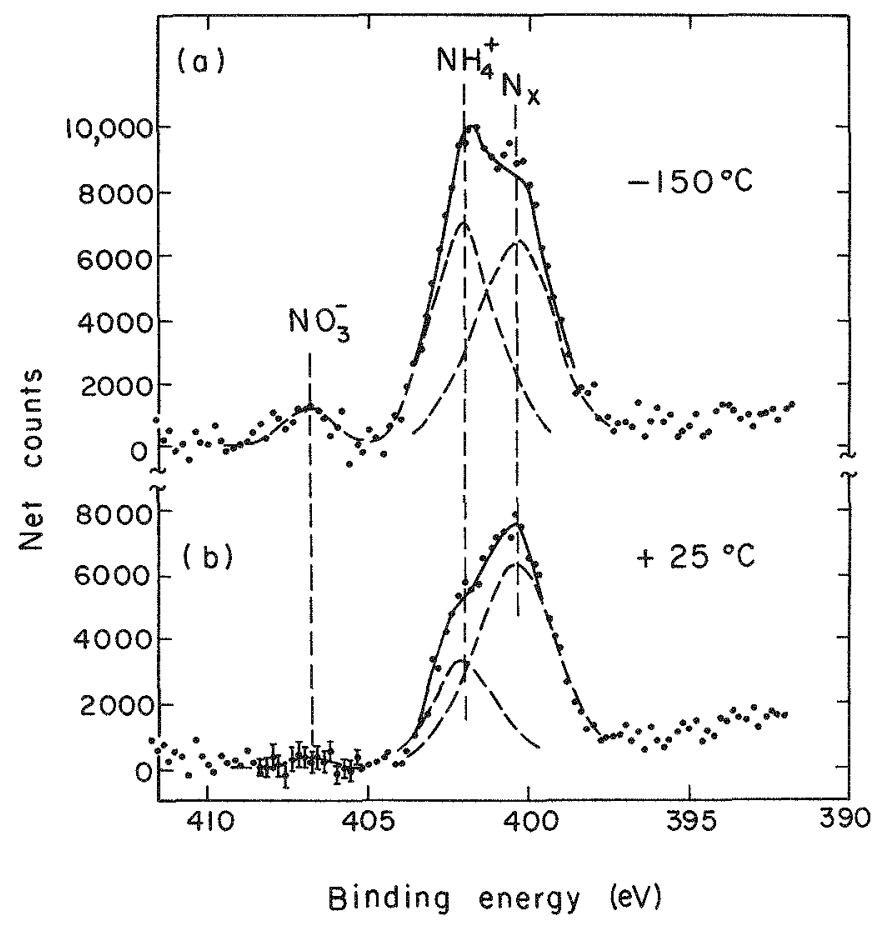

Fig. 1. (a) Nitrogen (1s) photoelectron spectrum of an ambient particulate sample with the specimen at $-150^{\circ} \mathrm{C}$. Individual peaks corresponding to $\mathrm{NO}_{3}^{-}, \mathrm{NH}_{4}^{+}$and $\mathrm{N}_{\mathrm{X}}$ are indicated. (b) The N(1s) spectrum of the sample sample at $25^{\circ} \mathrm{C}$.

(XBL 745-3183)

We conducted a number of experiments that show that surface reactions of soot particles with NO lead to the formation of both volatile ammonium and $N_{X}$ species, i.e., those that comprise much of the suspended nitrogen compounds observed in the ambient polluted air. Soot particles for these experiments were generated by a premixed propaneoxygen flame. The exposure of soot particles to the reactant gas was done under two different experimental conditions. In a "static" regime, with propane soot precollected on a filter subsequently exposed to the reactant gas at ambient temperature; and in a flow system, when the gas was introduced downstream from the flame, i.e., while the particles are at high temperature. 
ESCA spectra of $\mathrm{N}(1 \mathrm{~s})$ region of soot samples prepared in these ways are shown in Figs. 2 and 3. It is evident from Fig. 2 that interaction of NO with "cold" soot particles leads to the reduction of $\mathrm{NO}$ to $\mathrm{NH}_{3}$ which results in the formation of ammonium-like species, identical to those produced in soot $-\mathrm{NH}_{3}$ reaction. On the other hand, as seen from Fig. 3, both $\mathrm{NO}$ and $\mathrm{NH}_{3}$ interacting with "hot" soot particles produce species with binding energies corresponding to $\mathrm{N}_{\mathrm{X}}$ species.

Armonium species produced in NO-soot reaction have volatility properties in vacum similar to the ambient ammonium. The chemical equivalency of ambient and synthetic $N_{x}$ species can also be demonstrated by their thermal behavior. The experimental procedure involves the measurement of ESCA

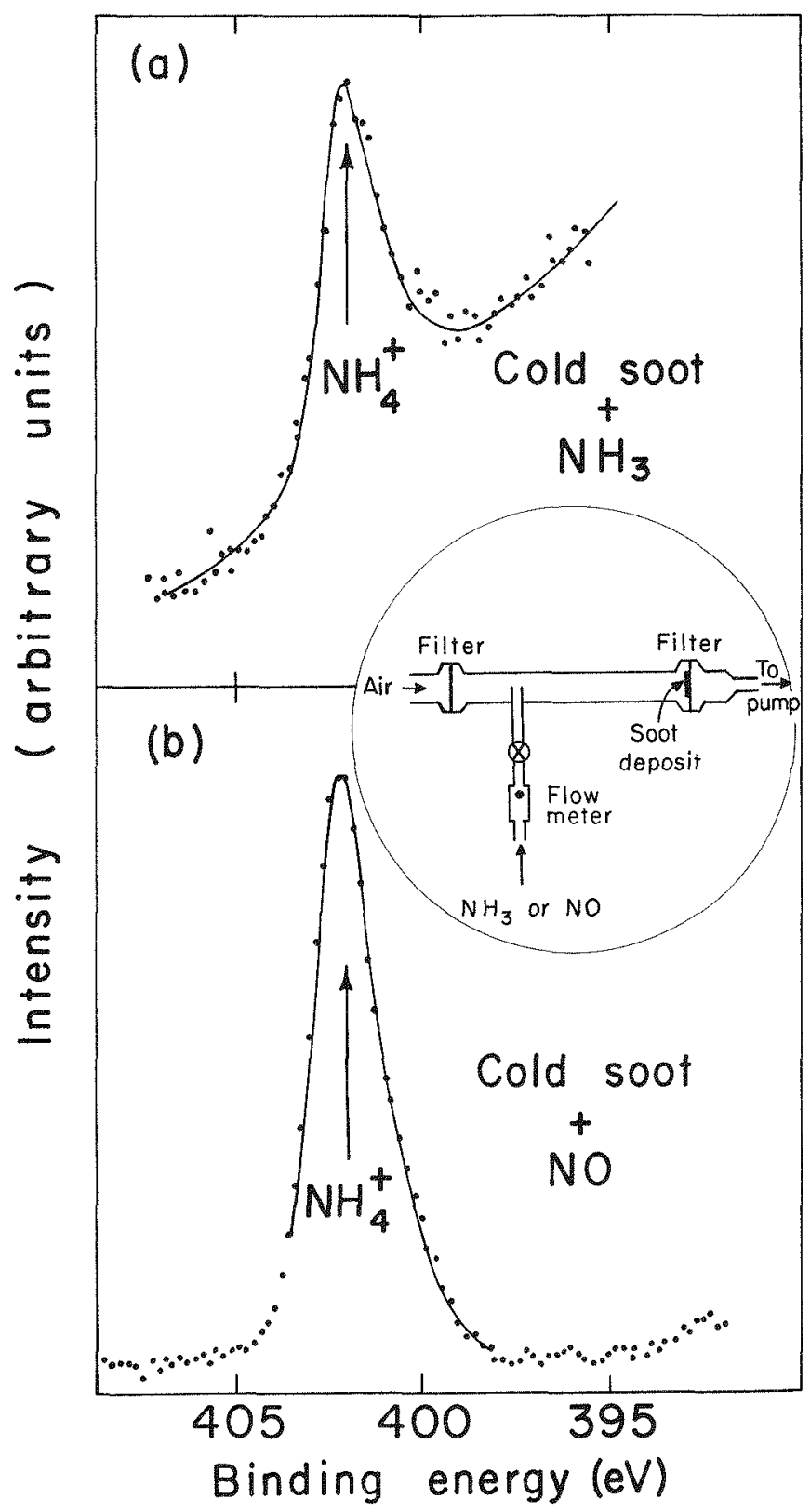

Fig. 2. (a) N(1s) spectrum of cold soot particles exposed to $\mathrm{NH}_{3}$. (b) $\mathrm{N}(1 \mathrm{~s})$ spectrum of cold soot particles exposed to NO.

(XBL 746-3530)

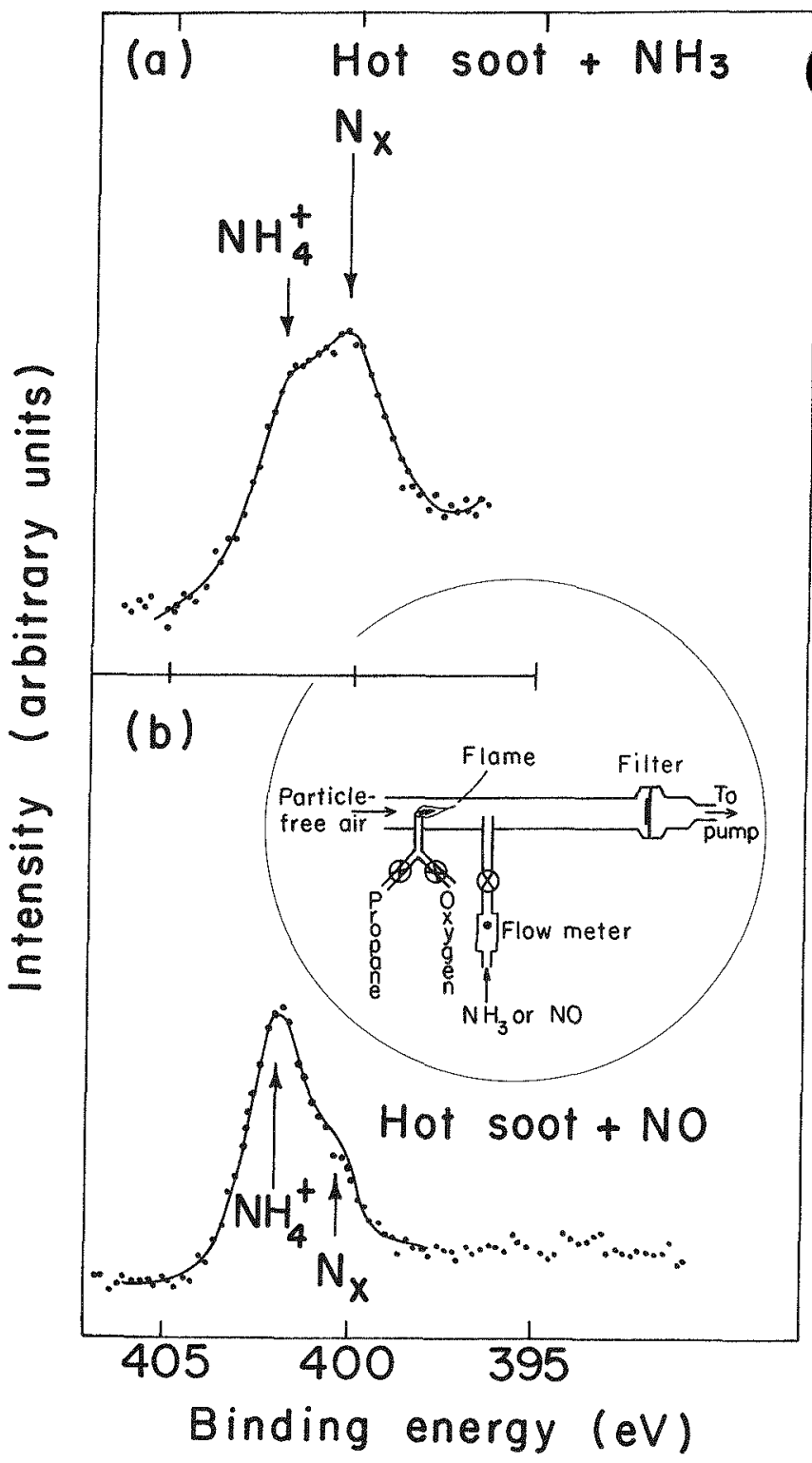

Fig. 3. (a) N(1s) spectrum of hot soot particles exposed to $\mathrm{NH}_{3}$. (b) $\mathrm{N}(1 \mathrm{~s})$ spectrum of hot soot particles exposed to No. Insert shows the experimental arrangement used for sample preparation.

(XBL, 746-3529)

spectra at gradua11y increasing temperatures. The results of such measurements for one ambient sample and for a sample prepared by $\mathrm{NH}_{3}$-hot soot interaction are shown in Figs. 4 and 5 . The spectrum of the ambient sample (Fig. 5) at $25^{\circ} \mathrm{C}$ shows the presence of $\mathrm{NO}_{3}^{-}, \mathrm{NH}_{4}^{+}$and $\mathrm{N}_{\mathrm{x}}$. At $80^{\circ} \mathrm{C}$ the entire $\mathrm{NO}_{3}^{-}$peak is lost accompanied with a similar loss in ammonium peak intensity (shaded portion). At $150^{\circ} \mathrm{C}$ the only nitrogen species remaining is $N_{x}$. (In this sample, the nitrate is mainly in the form of ammonia nitrate. The volatile ammoni is not seen because the initial temperature was $75^{\circ} \mathrm{C}$.) At $250^{\circ} \mathrm{C}$, the appearance of another peak, labeled $N^{\prime}$, is seen. This continues to increase at $350^{\circ} \mathrm{C}$. $\mathrm{X}_{\mathrm{X}}^{\prime}$ component is being formed from $\mathrm{N}_{\mathrm{X}}$ in the process of heating in vacum. $\mathrm{N}_{\mathrm{x}}$ species 


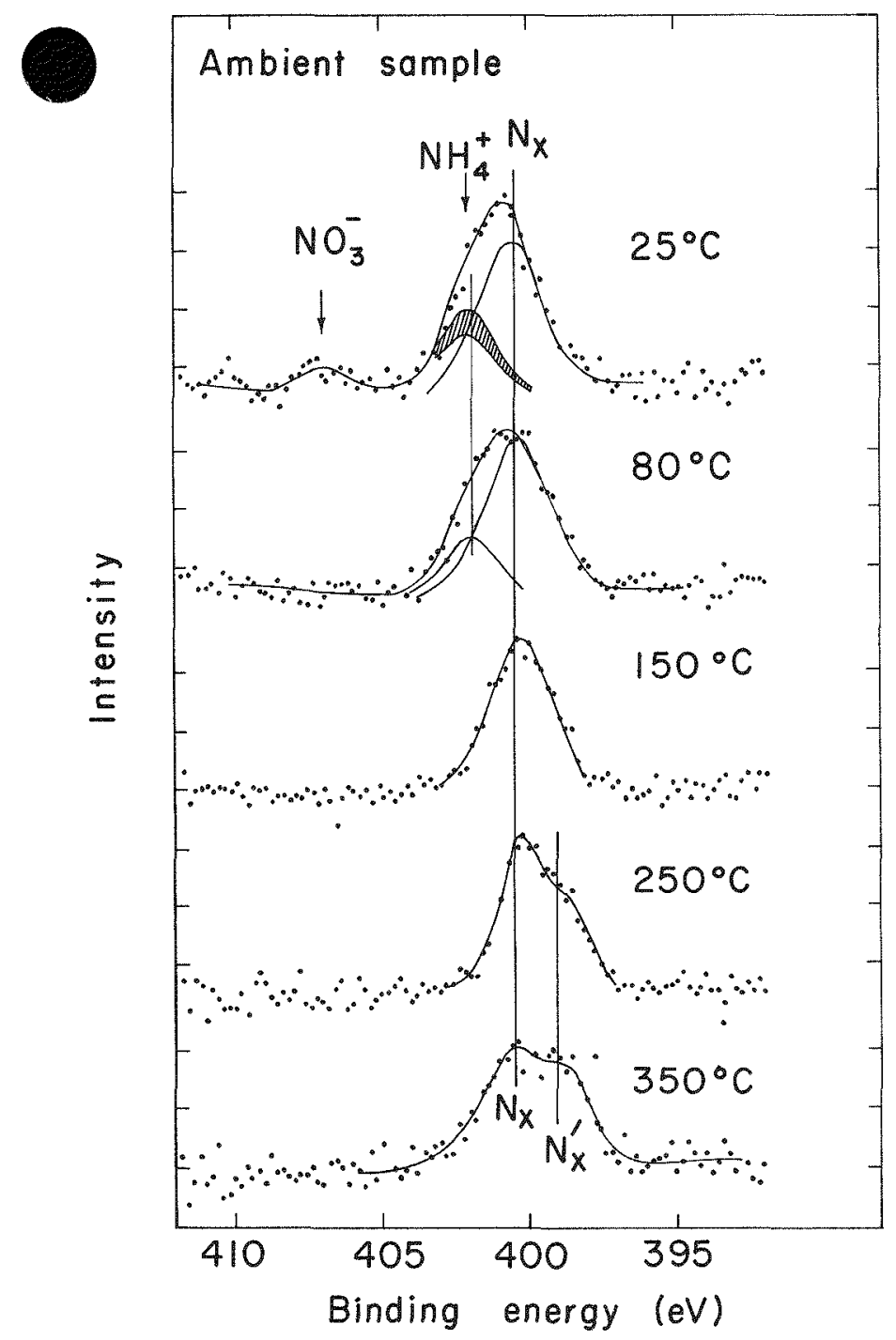

Fig. 4. N(1s) spectrum of an ambient sample exposed at $25^{\circ} \mathrm{C}, 80^{\circ} \mathrm{C}, 150^{\circ} \mathrm{C}, 250^{\circ} \mathrm{C}$ and $350^{\circ} \mathrm{C}$.

(XBL 746-3532)

produced by surface reactions of hot soot with NO (or $\mathrm{NH}_{3}$ ) have the same kind of temperature dependence as the ambient $N_{x}$ as illustrated in Fig. 5. Both ambient and synthetic $N^{\prime}$ species will remain unaltered even if the temperature is lowered back to room temperature, if the sample remains in vacuum. However, if the sample is taken out of vacuum and is exposed to moisture, $N_{x}^{?}$ will be transformed back to the original $N_{X}$. It can be concluded that $N_{X}^{\prime}$ species are produced by dehydration of $\mathrm{N}_{\mathrm{X}}$.

Our results suggest that the formation of amines and amides, i.e., the $\mathrm{N}_{\mathrm{x}}$ species, may occur in the combustion process where the temperature is high and where there are high concentrations of both NO and soot particles. $\mathrm{N}_{\mathrm{x}}$ species are thus expected to be primary pollutants emitted as such into the atmosphere. ${ }^{3}$ Actually, there is a strong correlation between the diumal concentration variations of $\mathrm{N}_{\mathrm{X}}$ and of total particulate carbon, suggesting the most of the particulate carbon is of

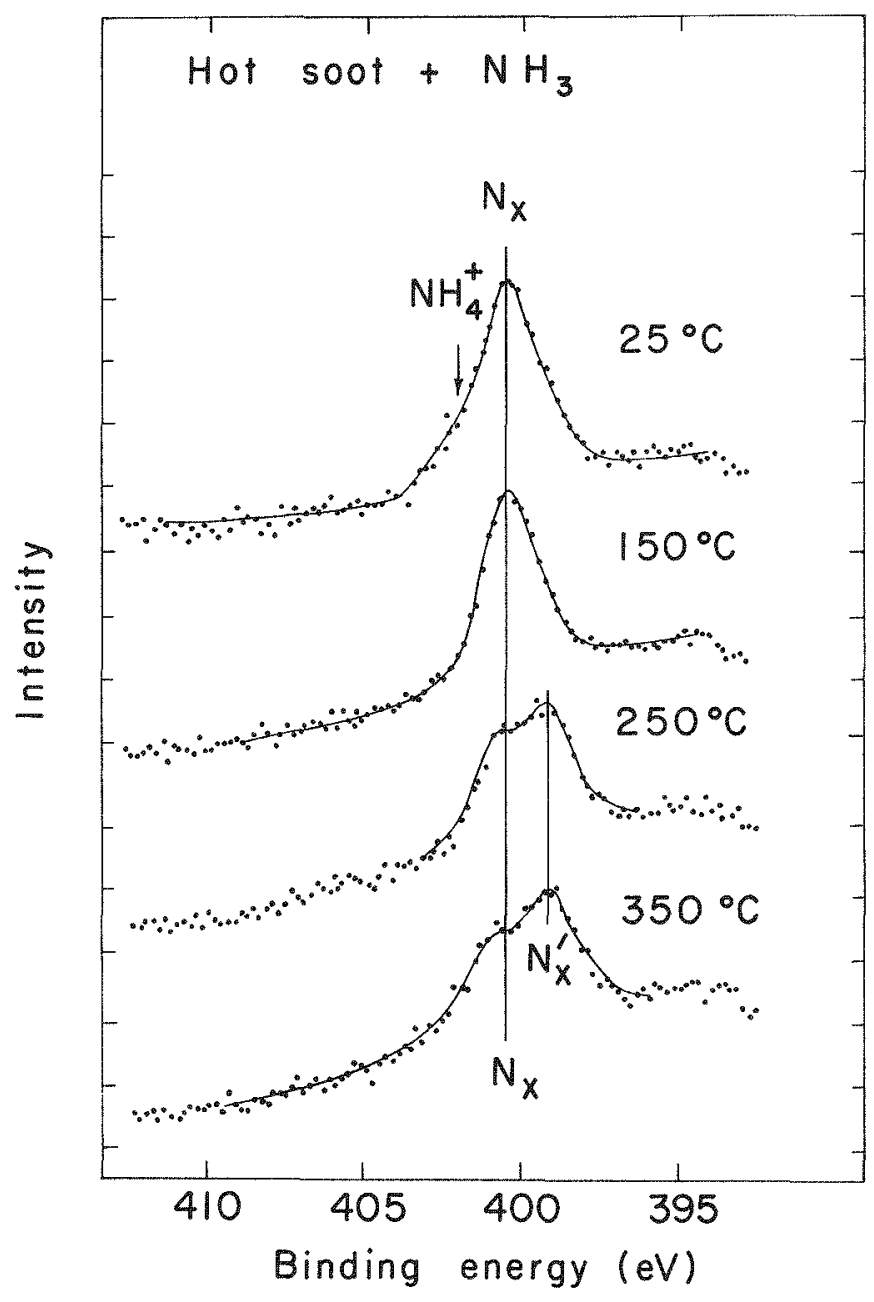

Fig. 5. N(1s) spectrum of (hot) soot sample exposed to $\mathrm{NH}_{3}$ as measured at $25^{\circ} \mathrm{C}, 150^{\circ} \mathrm{C}$, $250^{\circ} \mathrm{C}$ and $350^{\circ} \mathrm{C}$.

(XBL 746-3531)

primary origin. This is in contrast to some views, according to which most of the particulate carbon. is formed by condensation of the atmospheric reaction products of gaseous hydrocarbons. The conclusion about primary nature of $\mathrm{N}_{\mathrm{x}}$ species is further supported by the fact that both ambient and synthetic $N_{X}$ and $N_{x}^{\prime}$ are not volatile in vacuum up to at least $400^{\circ} \mathrm{C}$ and are not soluble in conventional solvents such as toluene. Furthermore, they do no dissociate in acidic solutions. If they were formed from homogeneous gas phase reactions, we would expect that the acid catalyzed hydrolys is of amide, nitrile or aromatic amine-type stoichiometric organic compounds to yield ammonium salts and carboxylic acids or aromatic alcohols.

The formation of volatile ammonium compounds of the hydroxyl or carboxyl ammonium type and/or adsorbed ammonia species is expected to occur at ambient temperatures; that is, along the pathways of primary carbon particles through the atmosphere.

The results described indicate the importance of carbon particles as sites for surface reactions with $\mathrm{NO}$ and $\mathrm{NH}_{3}$. We have already pointed out the 
importance of soot particles as a probable catalyst in $\mathrm{SO}_{2}$ oxidation to sulfate. 4

\section{Footnotes and References}

*Condensed from LBL-3068 (June 1974), Atmospheric Environment, 9, 495 (1975). Research supported by the Nationail Science loundation/RANN Program and the U.S.E.R.D.A.

1. T. Novakov, P. K. Mueller, A. E. Alcocer and J. W. Otwos J. Colloid Interface Sci., 39, p. 225, (1972).

2. B. R. Appe1, P. K. Mueller, J. J. Wesolowski, E. Hoffer, M. Fracchia, S. Twiss, S. Wa11, S. G. Chang and T. Novakov, Envir. Analyt. Chem., in press.

3. That $N_{X}$ species are indeed primary pollutants was experimentally verified by direct exhaust sampling of I.C. engines and jet turbine, for example.

4. T. Novakov, S. G. Chang, and A. B. Harker, Science, 186, p. 259, (1974).

DETERMINATION OF THE CHEMTCAL STATES OF SULFUR IN AMBIENT POLLUTION AEROSOLS BY X-RAY PHOTOELECTRON SPECTROSCOPY*

$$
\text { N. L. Craig, }{ }^{\dagger} \text { A. B. Harker }{ }^{\ddagger} \text { and T. Novakov }
$$

As the first step in a more comprehensive project dealing with heterogeneous atmospheric chemistry, we have undertaken a study whose aim is to establish a complete inventory of the sulfur compounds present in ambient particulate matter. This project was carried out through the study of a large number of pollution particulate samples using $X$-ray photoelectron spectroscopy (XPS.) as the analytical tool. Formulating such an "inventory" of ambient chemical species is a prem requisite for gaining an understanding of the atmospheric transformations of sulfur-containing compounds.

In the course of our work on the electron spectroscopy of ambient aerosols we have carefully examined the spectra of more than a hundred samples containing different particle size classes, collected at various sites in California at different times of day. (The aerosol samples were collected in the summer of 1972 during a California Air Resources Board sponsored study, "Characterization of Aerosols in California.") The sulfur XPS spectra were of varying degrees of complexity, sometimes covering the entire range of known sulfur binding energies. Individual binding energies, characteristic of definite sulfur species, were determined by a comparative study of a large number of specimens. These binding energies are assigned to characteristic chemical states, with the help of XPS results obtained from a number of simple sulfur compounds. Thanks to the measurable differences in binding energies between the bulk-type ions and the surface species, these can be distinguished in favorable cases.

The results of ambient sample analysis are presented in Table 1. Not all of the seven species which were observed occur generally at all times and at all locations. In most instances, sulfates are found to be the dominant species, although concentrations of reduced forms of sulfur were at times comparable to the sulfate concentrations.

From these results stem several implications of significance to both analytical and physical aeroso1 chemistry. For example, finding that sulfur species other than sulfates are often present in significant quantities makes it necessary to reexamine certain analytical methods for sulfate

Table 1. Sulfur 2p binding energies

\begin{tabular}{|c|c|c|c|c|c|c|}
\hline \multicolumn{2}{|c|}{$\begin{array}{l}\text { Ambient aerosol } \\
\text { samples }\end{array}$} & \multicolumn{3}{|c|}{$\begin{array}{l}\text { Surface species produced } \\
\text { by } \mathrm{SO}_{2} \text { and } \mathrm{H}_{2} \mathrm{~S} \text { adsorption }\end{array}$} & \multicolumn{2}{|c|}{$\begin{array}{l}\text { Sulfur } \\
\text { compounds }\end{array}$} \\
\hline $\begin{array}{l}\text { Peak } \\
\text { assignment }\end{array}$ & $\begin{array}{l}\text { Binding energy } \\
(\mathrm{eV})\end{array}$ & $\begin{array}{l}\text { Species } \\
\text { designation }\end{array}$ & $\begin{array}{l}\text { Binding energy } \\
(\mathrm{eV})\end{array}$ & System & Compound & $\begin{array}{l}\text { Binding energy } \\
(\mathrm{eV})\end{array}$ \\
\hline A sulfate & $169.2 \pm 0.2$ & $\mathrm{SO}_{4}^{2-}$ & $169.7 \pm 0.2$ & $\mathrm{MgO}+\mathrm{SO}_{2}$ & $\mathrm{Na}_{2} \mathrm{SO}_{4}$ & $169.0 \pm 0.3$ \\
\hline $\mathrm{B} \mathrm{SO}_{3}$ & $170.6 \pm 0.3$ & $\mathrm{SO}_{3}$ & $171.0 \pm 0.3$ & $\mathrm{MgO}+\mathrm{SO}_{2}$ & & \\
\hline $\mathrm{C} \mathrm{SO}_{2}$ & $168.1 \pm 0.3$ & $\mathrm{SO}_{2}$ & $167.9 \pm 0.3$ & $\mathrm{MgO}+\mathrm{SO}_{2}$ & & \\
\hline D Sulfite & 167 & & & & $\mathrm{Na}_{2} \mathrm{SO}_{3}$ & $167.0 \pm 0.3$ \\
\hline E elem. S & $164.2 \pm 0.3$ & & & & $S$ & 164.2 \\
\hline F sulfide & $162.6 \pm 0.3$ & & & & $\mathrm{ZnS}$ & $162.2 \pm 0.3$ \\
\hline G sulfide & $160.5 \pm 0.6$ & $\mathrm{~s}^{2-}$ & $161.0 \pm 0.3$ & $\mathrm{CaO}+\mathrm{H}_{2} \mathrm{~S}$ & & \\
\hline
\end{tabular}




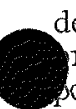
etermination. Some of these methods implicitIy resume that the sulfates are the only sulfur comounds present in the aerosol samples. Methods based on the reduction of sulfates to $\mathrm{H}_{2} \mathrm{~S}$ are in this category.

\section{Footnotes}

* Condensed from Atmospheric Environment 8, 15 (1974)
LBL-1584. Work supported by the National Science Foundation RANN Program, the California Air Resources Board under a contract with California State Dept. of Health, and the USERDA.

Tresent address: Dept. of Chemistry, University of Georgia, Athens, GA 30601

† Present address: Science Center, Rockwe11 Internationa1, Thousand Oaks, CA 91360

\section{SULFATES AS POLLUTION PARTICULATES: CATALYTIC FORMATION ON CARBON (SOOT) PARTICLES*}

T. Novakov, S. G. Chang and A. B. Harker ${ }^{\dagger}$

Because of the adverse effects of sulfate particles as atmospheric pollutants, the study of the oxidation of $\mathrm{SO}_{2}$ to sulfate is of prime importance in air pollution research. In the past, most attention has been devoted to the study of the photochemical and solution chemical oxidation mechanisms. There is increasing evidence, however, that these two kinds of processes alone cannot adequately account for the observations. This report concerns the role that finely divided carbon (soot) particles play in the oxidation of $\mathrm{SO}_{2}$. Even though the surface chemical properties of carbon are known, 1,2 the relevance of sootcatalyzed reactions to air-pollution chemistry has not been appreciated. The experiments described below show that the soot-catalyzed oxidation of $\mathrm{SO}_{2}$ to sulfate is an important process and that the proposed oxidation mechanism is in qualitative agreement with the field observations.

Soot specimens from a premixed $\mathrm{C}_{3} \mathrm{H}_{8}-\mathrm{O}_{2}$ flame collected on (silver membrane) filters were used for experiments with different $\mathrm{SO}_{2}$ exposure conditions, in the apparatus shown in Fig. 1. Dry air or prehumidified particle-free air or $\mathrm{N}_{2}$ was used with an $\mathrm{SO}_{2}$ concentration of about $300^{2}$ parts per million (ppm) and an exposure time of five minutes. The ESCA spectra of soot exposed to $\mathrm{SO}_{2}$ are shown in Fig. 1. The sulfate peaks were always more intense in the case of prehumidified air than in the case of dry air. However, both dry and prehumidified $N_{2}$, when used instead of air, produced only very low, background level sulfate peaks. This result indicates that, in addition to soot particles, the $\mathrm{O}_{2}$ in air is important for $\mathrm{SO}_{2}$ oxidation. Although water molecules enhance the observed sulfate concentration in the air- $\mathrm{SO}_{2}$-soot system, the contribution of sulfate produced by $\mathrm{SO}_{2}$ oxidation via dissolved molecular oxygen in water droplets is not significant; that is, blank filters exposed to $\mathrm{SO}_{2}$ and prehumidified air showed at most only low, background level sulfate peaks.

We have also studied the $\mathrm{SO}_{2}$ oxidation on soot particles produced in the premixed $\mathrm{C}_{3} \mathrm{H}_{8}-\mathrm{O}_{2}$ ame by observing the decrease in the gaseous 2 concentration, $\Delta\left(\mathrm{SO}_{2}\right)$, occurring as a result sulfate formation (Fig. 2). The $\mathrm{SO}_{2}$ concentration is adjusted to the desired initial value, $\left(\mathrm{SO}_{2}\right)_{i}$, with the flame removed from its position in front of the intake funnel. A decrease in the $\mathrm{SO}_{2}$ concentration is observed within the response

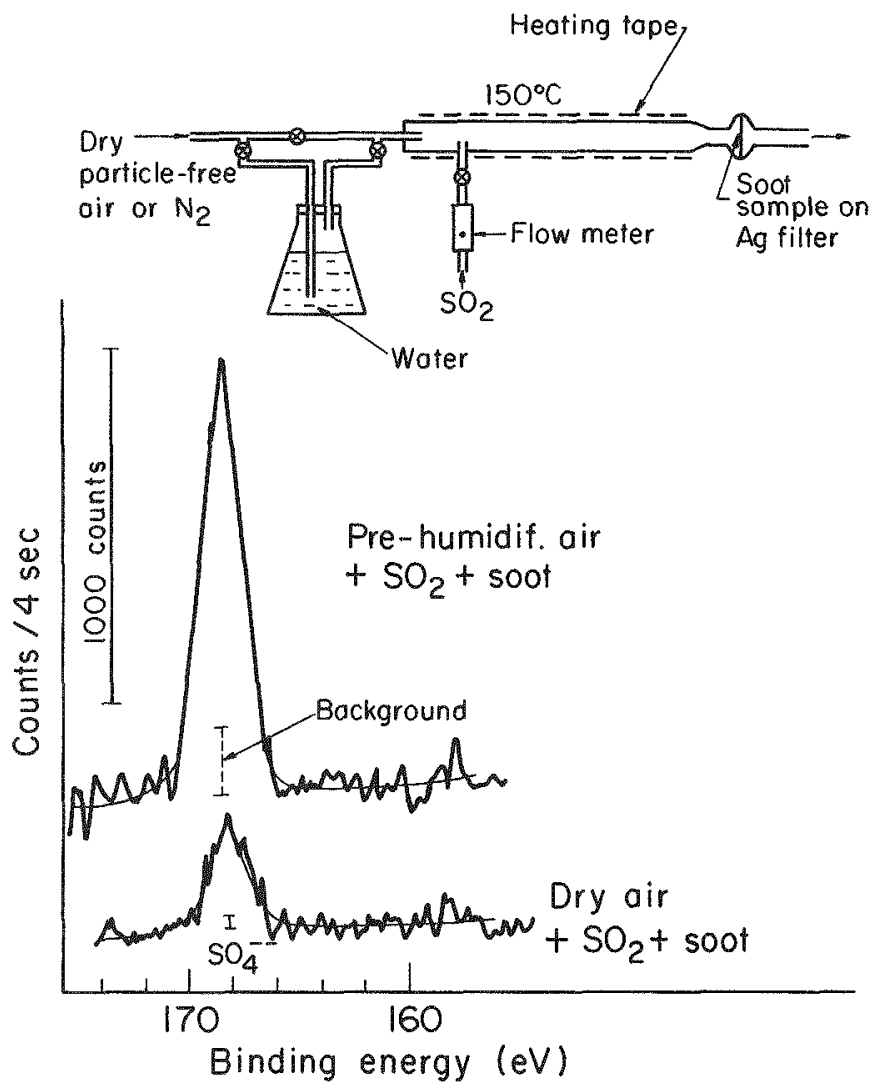

Fig. 1. Soot particles exposed to prehumidified air and $\mathrm{SO}_{2}$ produce sulfate concentrations higher than in the case of dry air. Blank filters without soot particles exposed to $\mathrm{SO}_{2}$ and prehumidified air show only background level sulfate.

(XBL 743-2503)

time of the $\mathrm{SO}_{2}$ monitor, when the flame is placed in the intake position so that combustion-generated aerosol comes in contact with $\mathrm{SO}_{2}$. Removal of the flame causes the $\mathrm{SO}_{2}$ concentration to rise to its initial value. Because gaseous species will suffer about $10^{8}$ coliisions on the path between the flame and the $\mathrm{SO}_{2}$ input, it can be expected that reactive radical species will be largely neutralized by the time they reach the $\mathrm{SO}_{2}$ port. This and the evidence described earlier suggests that the observed decrease in the $\mathrm{SO}_{2}$ 

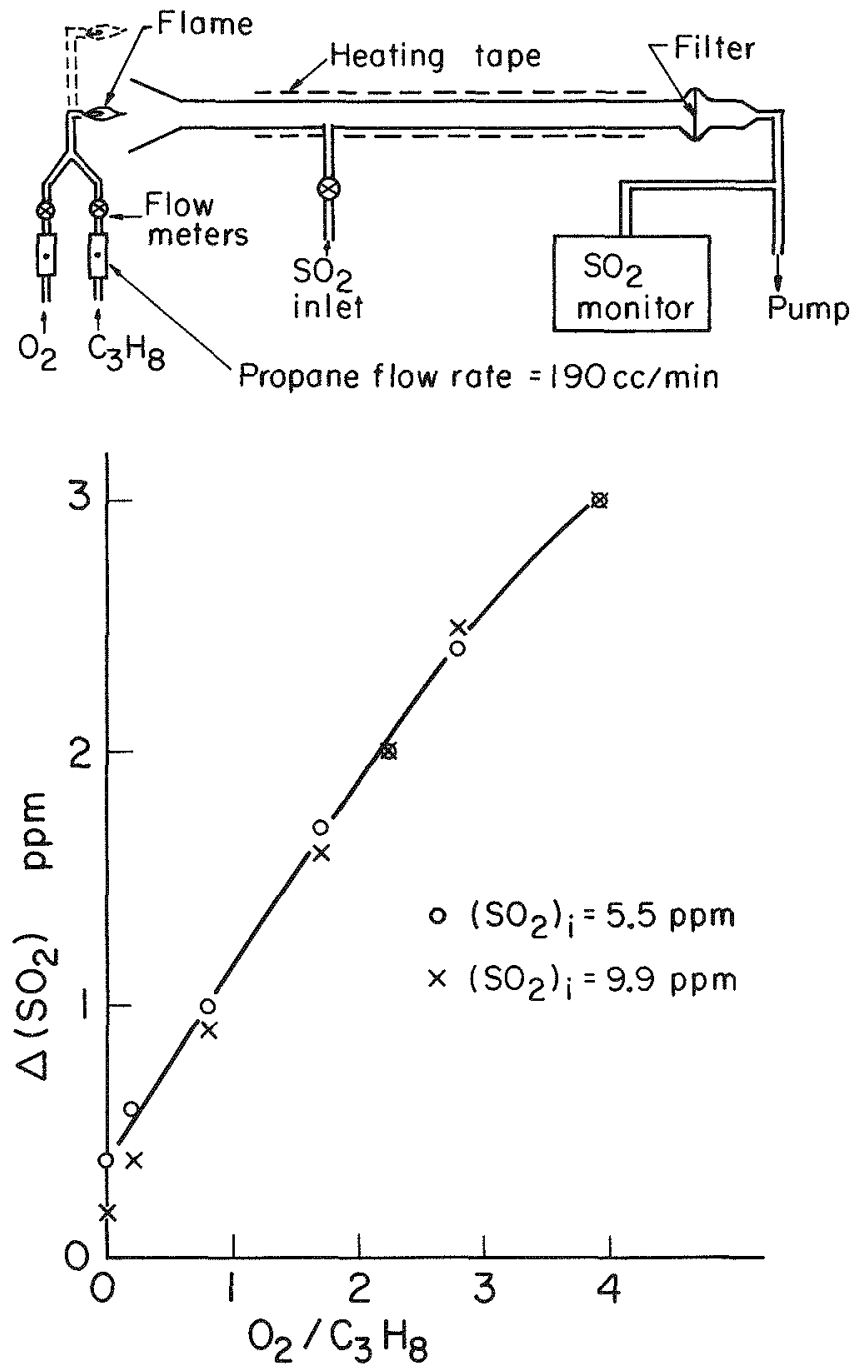

Fig. 2. $\mathrm{SO}_{2}$ concentration is adjusted to the desired value $\left(\mathrm{SO}_{2}\right)$, with the flame removed from its position in front of the intake funnel. Decrease in $\mathrm{SO}_{2}$ concentration, $\Delta\left(\mathrm{SO}_{2}\right)$, is observed when the flame is placed in the intake position. For a given combustion regime $\Delta\left(\mathrm{SO}_{2}\right)$ is independent on $\left(\mathrm{SO}_{2}\right)_{i}$, while $\Delta\left(\mathrm{SO}_{2}\right)$ increases with $\mathrm{O}_{2} /$ fuel ratio. The former effect is related to the saturation of active sites on soot particles, while the latter reflects the increase in the number of reactive particles.

(XBL 743-2504)

concentration is caused by the action of soot particles. The possibility that the reduction in the $\mathrm{SO}_{2}$ concentration is related to the formation of $\mathrm{H}_{2} \mathrm{SO}_{3}$ was ruled out in a separate experiment, in which the combustion-generated aerosol was replaced by steam. No detectable change in the $\mathrm{SO}_{2}$ concentration was observed in this case.

The plot in Fig. 2 shows the amount of $\mathrm{SO}_{2}$ converted to sulfate as a function of the $\mathrm{O}_{2} / \mathrm{C}_{3} \mathrm{H}_{8}$ ratio, for initial concentrations of 5.5 and $9.8 \mathrm{ppm}$. For a given combustion regime $\Delta\left(\mathrm{SO}_{2}\right)$ is independent of $\left(\mathrm{SO}_{2}\right)_{i}$. This feature is probably related to the saturation of active sites on soot particles. The $\Delta\left(\mathrm{SO}_{2}\right)$ value increases, however, with the $\mathrm{O}_{2} / \mathrm{C}_{3} \mathrm{H}_{8}$ ratio, reflecting the increase in the number of very sma11, high-surfacearea particles produced in oxygen-rich flames.

That the sulfate formation process described here is" consistent with field observations is evident from the following. Pollution particulate sulfates are believed to exist primarily in the form of $\mathrm{H}_{2} \mathrm{SO}_{4}$ or $\left(\mathrm{NH}_{4}\right)_{2} \mathrm{SO}_{4}$, or both. Because the sulfate produced by the laboratory soot- $\mathrm{SO}_{2}$ interaction is water-soluble and could conceivably be neutralized by ambient $\mathrm{NH}_{3}$, its chemical properties are consistent with those of ambient sulfate in the analytical sense. Moreover, ambient and laboratory-produced sulfate exhibit the same characteristic desorption in a vacuum as a function of sample temperature. The saturation effect reported for ambient sulfates ${ }^{3}$ is also consistent with the proposed process. Finally, a marked correlation between the diurnal variation in the concentrations of ambient carbon and sulfate should be expected. An example of such a correlation is shown in Fig. 3. Other similar correlations have been observed more recently for other sites and pollution episodes. 4

The catalytic formation of sulfate on soot particles is expected to occur in the open atmosphere and especially in or near combustion sources, where both $\mathrm{SO}_{2}$ and soot concentrations are highest.

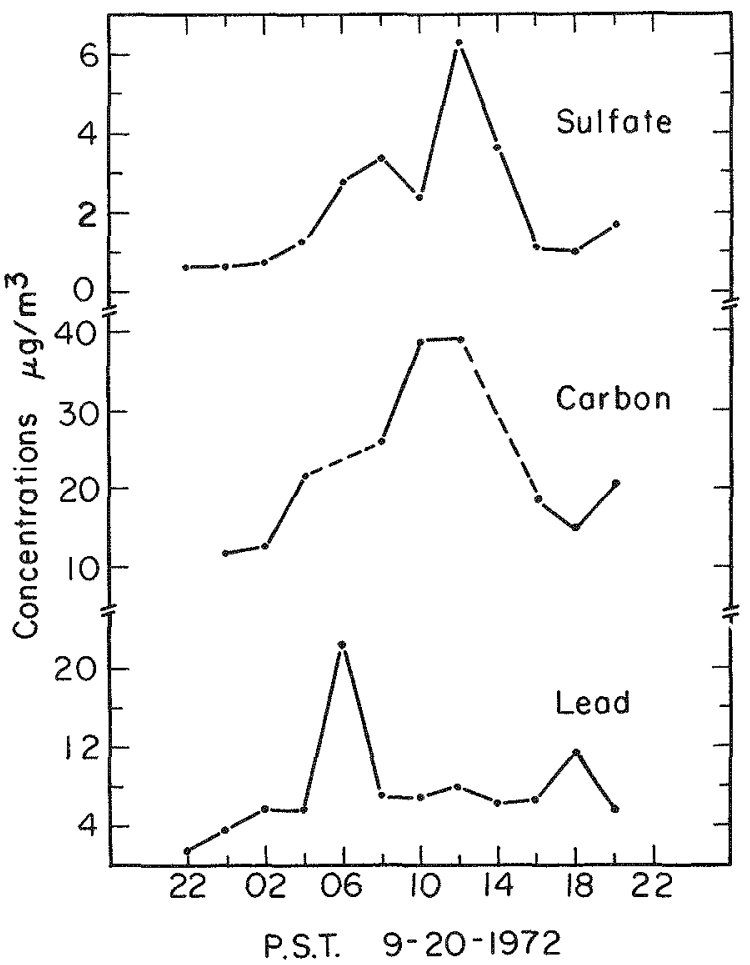

Fig. 3. Diurnal concentration variation of sulfate particulate carbon (ref. 5), and lead (ref. 6). The sampling was done in downtown Los Angeles on 20 September, 1972 (ref. 7). The similarity between carbon and sulfate patterns is obvious.

(XBL 743-2502) 
Footnotes and References

Condensed from Science, 186, 259 (1974), LBL-3077. Research supported by the National Science Foundation/RANN Program and the USERDA.

†Present Address: Science Center, Rockwe11 Internationa1, Thousand Oaks, California 91360.

1. M. Com and R. T. Chang, J. Air Pollut. Control Assoc., 22, 870 (1972).

2. R. N. Smith, Quart. Rev., 13, 287 (1959).

3. A. P. Altshulier, Environ. Sci. Tech., 7, 709 (1973).
4. G. M. Hidy, B. R. Appe1, W. E. Clark, S.K. Friedlander, J. Holmes, T. Novakov, P. Roberts and J. J. Wesolowski, Presented at the American Chemical Society Meeting, Division of Environmental Chemistry, Los Angles, California, 31 March - 5 April, 1974.

5. P. Colodny and B. R. AppeI, unpublished.

6. R. D. Giauque, unpublished.

7. "ACHEX," Aeroso1 Characterization Program of the California Air Resources Board.

\title{
DISTRIBUTION OF TRACE ELEMENT AEROSOLS IN URBAN AIR
}

\author{
W. Winklestein, Jr., S. Sacks, and R. D. Giauque
}

Concern with the disease producing effects of ambient aerosols was stimulated in the United States by the Donora Pennsylvania air pollution episode of October, 1948.1 Since then both laboratory and field studies have shown that a wide variety of disease-producing agents are disseminated in air pollution. However, for largely technical reasons it has been difficult to identify routinely more than a very few components of particulate air pollution such as sulphates and a few metals and organics. Furthermore, these pollutants frequently occur together in the air. Thus the demonstration of associations between fluctuations of particulate air pollution and mortality and morbidity have not been attributable to specific pollution components. ${ }^{2}$ Very 1 ittle effort has been made to relate the components of ambient air to body burden.

Recently, techniques such as $x$-ray fluorescence for the rapid identification of a wide spectrum of elemental substances have been developed. This provides investigators with a powerful tool for assessing and monitoring ambient air concentrations of potentially toxic materials. Making use of this development, we have used samples of particulate air pollution obtained from 18 sampling stations in and around Buffalo, New York, between July, 1961 and June, 1963 to ascertain the ambient air levels for 15 elements. Applying computer mapping programs, we have established isopleths for each measured element over the study community. As far as we know, this is the first time such information has become available for a large community.

The samples were obtained in a study of air pollution effects that demonstrated an association between suspended particulate levels and total mortality, as we11 as mortality from chronic respiratory disease, gastric cancer, prostatic cancer, and cirrhosis of the liver, independent of economic class. 3 This study also demonstrated an association between particulate air pollution and lower respiratory system symptoms in nonsmokers. The sampling is accomplished by the use of high volume partihlate samplers operated on random 24-hour schedules so that each station provided approximately 200 samples over the two year period with equal representation of each day of the week. The particulates were deposited on glass fiber filters, which were at that time the standard available material for high volume sampling. Using the unexposed margins of the original filters, background levels for the 15 elements tested were determined by $x$-ray fluorescence. The particulates were then analyzed and the filter background levels subtracted from each determination.

In Table 1 the range of medians for each of the 15 measured elements over a11 18 sampling stations is shown. In addition the total suspended particulate values for each station are given. It is apparent from Table 1 that there are wide variations in ambient air concentrations of the 15 measured elements. Each element varies considerably in concentration between sampling stations as well as at each station. It is not surprising that the highest concentrations recorded are for iron ( $\mathrm{Fe}$ ) since the study area includes one of the larger steel mills in the United States. The lowest concentrations recorded are for arsenic, selenium and gallium. Of the 15 elements measured, three are thought to cause cancer in humans: arsenic,

Table 1. Ranges of median values over all sampling stations by element (mg/m $324 \mathrm{hr}$ )

\begin{tabular}{lrr}
\hline & Low & High \\
\hline Nicke1 & 14 & 35 \\
Copper & 54 & 204 \\
Arsenic & 0 & 21 \\
Rubidium & 5 & 20 \\
Selenium & 7 & 13 \\
Strontium & 5 & 55 \\
Calcium & 1682 & 4274 \\
Lead & 494 & 1000 \\
Bromine & 39 & 143 \\
Potassium & 316 & 1189 \\
Zinc & 319 & 937 \\
Iron & 1838 & 21,368 \\
Manganese & 75 & 507 \\
Galiium & 6 & 14 \\
Chromium & 39 & 123 \\
Suspended & & \\
$\quad$ Particulates & 75,000 & 140,000 \\
(ng/m/24 hrs.) & & \\
\hline \hline
\end{tabular}


chromium, and nickel. of the remaining elements, the most important known disease agent is lead.

A persistent problem in evaluating the $d i$ sease-producing effects of ambient air pollution has been the inability to separate particular components of pollution in order to isolate specific effects. We have calculated rank order correlation coefficients between each of the 15 measured elements as we11 as total suspended particulates in. order to assess the potential for evaluating specific effects. The correlation coefficients were computed by ranking the median values for each station for each of the elements measured and then calculating the Spearman rank order correlation coefficients for all combinations of two elements. of the 120 rank order correlations calculated (in absolute value), five have coefficients 0.800 or greater, 24 have coefficients 0.6 to $0.799,39$ have coefficients 0.4 to 0.599 , and 52 have coefficients less than 0.4. The lower the correlation coefficients the greater is the opportunity to evaluate independent effects. In Table 2, we have selected several putative disease agents and have tabulated their rank order correlative coefficients. The elements chosen are copper, iron, lead, nicke1, zinc.

Table 2. Rank order correlation coefficients

\begin{tabular}{lccccc}
\hline & Copper & Iron & Lead & Nicke1 & Zinc \\
\hline Copper & 1 & 0.25 & 0.37 & 0.42 & 0.18 \\
Iron & & 1 & 0.49 & 0.52 & 0.58 \\
Lead & & & 1 & 0.68 & 0.56 \\
Nicke1 & & & & 1 & 1 \\
Zinc & & & & \\
\hline
\end{tabular}

In order to illustrate more clearly the distribution of aerosols over the entire community, isopleths have been generated for each element analyzed. An example of such isopleths showing the distribution of nickel (a putative carcinogen) is given in Fig. 1. It is now apparent that values for each measured aerosol can be assigned to each census tract, permitting a variety of analytic procedures to be applied to test for associations with particular disease occurrence or body burdens.

In order to partially validate the mapping procedure, we sequentially removed one station at a time from the analyses and evaluated the resulting effect on the contour pattern and the predicting value of that station. In accordance with expectation, deletion of peripheral stations distorts the outermost contours by a considerable amount. Removal of interior stations has a minor effect except where these stations are close to a high peak or low valley as regards pollutant level. In general, removal of an interior station produces a change of about $10-20 \%$ in the value of nearby contours. Thus, the values of the inner contour are not overwhelmed by removal of a single sampling point. These results have given us confidence in the validity of our approach.

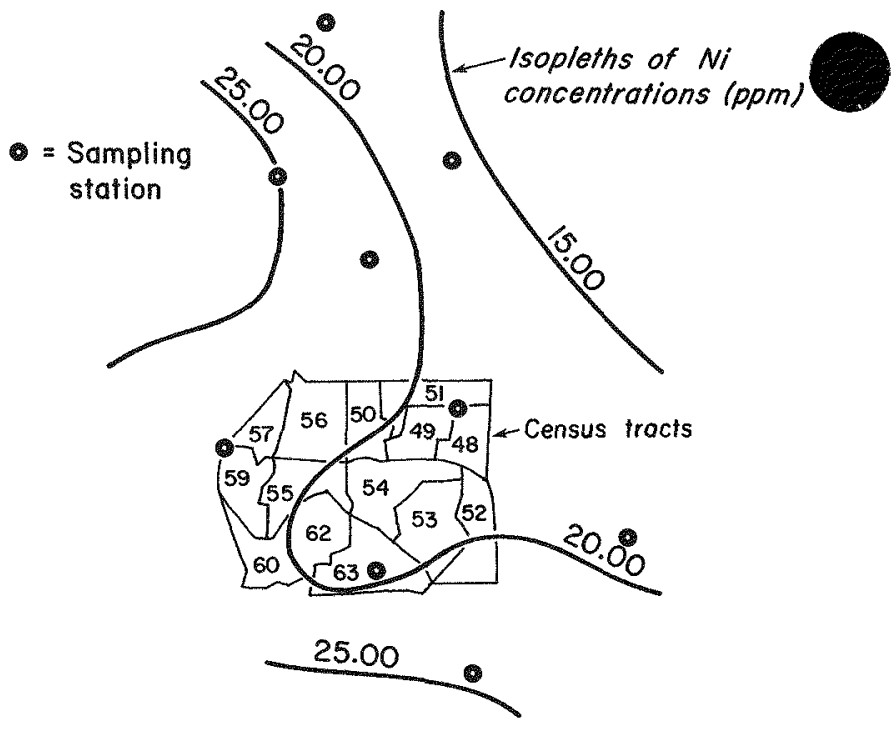

Fig. 1. Example (somewhat abridged) showing distribution of nickel aerosols in Erie County. A few census tracts are shown, demonstrating the possibility of correlating census data with the aeroso1 distributions. (XBL 761-2012)

A recent analysis carried out by Professor Carl A. Keller, University of California at Berkeley, demonstrates how the specific pollutant exposure values for each census tract can be evaluated. He used a stepwise multiple regression analysis with 22 independent variables, including suspended particulate levels for each census tract. The response variable used was mortality from all causes adjusted for age, race and sex. other independent variables included census tract indices of income, mobility, nativity, employment status, education, population density, quality of housing, and family structure. This analysis revealed that suspended particulates was included among the four or five variables that had regression coefficients significantly different from zero. other significant independent variables were median family income, educational status, housing quality and family structure. An example of the data is shown in Table 3 . By adding the various elements identified by $x$-ray fluorescopy to the multiple regression analysis we hope to be able to evaluate their independent effects.

Perhaps the most significant potential use of the procedures described above is the possibility of relating body burdens of a wide variety of elements to ambient air exposures. By using a higher energy source in the $x$-ray fluorescence analysis systern, a much wider spectrum of elements can be identified. Furthermore, it may also be possible to apply automated gas chromatography with mass spectroscopy to measure polynuclear. aromatic hydrocarbons and other related organics so that similar assignment of census tract exposure values can be accomplished. The application of this advanced technology to the study of air pollution may provide the key to the more accurate evaluation of the disease-producing effects of ambient air pollution. 
Table 3. Stepwise Multiple Regression - Summary Table. Dependent Variable - Mortality from all causes, white males, 50-69.

\begin{tabular}{|c|c|c|c|}
\hline $\begin{array}{c}\text { Step } \\
\text { number }\end{array}$ & $\begin{array}{l}\text { Variable } \\
\text { entered }\end{array}$ & $\begin{array}{l}\text { F value } \\
\text { to enter }\end{array}$ & Significance \\
\hline & Dilapidated Housing & 91.2966 & $\mathrm{P}<0.01$ \\
\hline & Median Income & 29.3097 & $\mathrm{P}<0.01$ \\
\hline & Suspended Part. Level & 17.8559 & $\mathrm{P}<0.01$ \\
\hline & Median Years Education & 14.5543 & $\mathrm{P}<0.01$ \\
\hline & Semi-Crowded Living & 3.3008 & ns \\
\hline & Poverty Income & 1.7634 & ns \\
\hline & Dismuptive Marriage & 2.4000 & ns \\
\hline & Multiple Housing & 3.0086 & ns \\
\hline & Deteriorated Housing & 1.2938 & ns \\
\hline & Per Capita Income & 1.4490 & ns \\
\hline & Unrelated Household & & \\
\hline & Members & 1.3869 & ns \\
\hline & Males in Labor Force & 1.4399 & ns \\
\hline & Foreign Born & 1.0001 & ns \\
\hline & Rental Housing & 0.3304 & ns \\
\hline & Crowded Living Conditions & 0.4575 & ns \\
\hline & Females in Labor Force & 0.4349 & ns \\
\hline & Non-White & 0.1613 & ns \\
\hline & Broken Marriages & 0.3983 & ns \\
\hline & Moved Into Tract Within & & \\
\hline & $\begin{array}{l}5 \text { Years } \\
\text { No Bathroom }\end{array}$ & $\begin{array}{l}0.0120 \\
0.0667\end{array}$ & $\begin{array}{l}\text { ns } \\
\text { ns }\end{array}$ \\
\hline & No Hot Water & 0.1616 & ns \\
\hline & Unemployed & 0.0669 & ns \\
\hline
\end{tabular}

\section{REFERENCES}

1. H. H, Shrenk, H. Herman, G. D. Clayton, et a1., "Air Pollution in Donora, Pa., Epidemiology of the Unusual Smog Episode of October 1948, "Preliminary Report Published in Health Bulletin 306 (1949).

2. W. Winkelstein, Jr., and M. Gay, "Arteriosclerotic Heart Disease and Cerebrovascular
Disease: Further Observations on the Relationship of Suspended Particulate Air Pollution and Mortality in the Erie County Air Pollution Study," in Proceedings of the 16 th Annual Meeting of the Institute of Environmental Science, Boston, 1970, pp. 441-447.

3. W. Winkelstein, Jr., S. Kantor, E. W. Davis, et a1. Arch. Env. Health 14, 162 (1967).

\section{CADMIUM LEVELS IN THE SHORELINE SEDIMENTS OF SAN FRANCISCO BAY*}

B. R. Moyer and T. F. Budinger

\section{INTRODUCTION}

The San Francisco Bay is a likely candidate for possible cadmium contamination because it is in an area of increasing population, high sewage discharge, industrial effluent, and limited oceanic dilution.1,2 our purpose for investigating the San Francisco Bay is twofold: (1) to establish quantitative background levels which can set a base for future environmental monitoring, and (2) to identify the gross soil and environmental characteristics of potential areas of serious tadmium accumulation.

River waters that are polluted with cadmiun often show low, even undetectable, levels of the metal while large concentrations can be found in suspended particles and bottom sediments. This is especially true in waters of neutral or alkaline $\mathrm{pH}$. For example the water concentration downstream from a cadmium refinery might be only $4 \mathrm{ppb}$, whereas the bottom sediments will show 80,000 ppb.1,3 Thus area pollution assessments can be very much in error unless deposition in soils and sediments are taken into account.

The San Francisco Bay has an area of approximately 400 square miles and occupies a depression formed by tectonic processes. The northern section of the bay, including San Pablo Bay, obtains almost all the fresh water inflow, and this fresh water accounts for $40 \%$ of the entire land mass drainage in the State of California. The southern section of the bay has a limited influx of fresh water and thus a potentially more serious pollutant accumulation and disposal problem. 4 Most of the 
bay is less than 30 feet deep with the exception of navigation channels dredged to depths of 70 feet.

The total gross heavy metal disposal into the bay is estimated at 11 tons per day. 5,6 Lead levels in the bay muds are 20 to $30 \mathrm{ppm}$ while zinc and iron are generally 14 to $1800 \mathrm{ppm}$ and 10,000 respectively. 5 Levels for cadmium in the muds have not been previously reported.

Sediment accretion has been continuous in recent times and about one-third of the original bay has been taken up by refuse dumping or sanitary land fill. Assuming a maximum sediment accretion rate of $2 \mathrm{~mm}$ per year with no equilibration by tidal washout of the sediment, the naturally accrued level of sediment since 1920 should approximate 10 $\mathrm{cm}$. Each core sample examined in this study was taken to a depth of $10 \mathrm{~cm}$ and then divided into a top fraction and a bottom fraction $(1-7 \mathrm{~cm}$ and 7-10 cm respectively).

\section{RESULTS AND DISCUSSION}

Samples of sediment were analyzed by atomic absorption, and the distribution of the cadmium concentrations found in top and bottom fractions of the representative 10 -cm cores around the bay is shown graphically in Fig. 1. The first and major mode is 0.6 to 0.79 and represents $27 \%$ of a11 the combined top and bottom fractions. Eighteen were above the $2.0 \mathrm{ppm}$ d.wt. level of the 136 samples $(13 \%)$ and we suspect these represent cadmium accumulation above natural background leve1s. Further evidence of "recent" cadmium accumulation in the bay sediments can be seen in Fig. 2 where a flattening of the Gaussian distribution for the top fractions (top $7 \mathrm{~cm}$ ) can be seen relative to the bottom fractions (bottom $3 \mathrm{~cm}$ ) distribution curve. To explore the origin of this difference, we divided the bay into four sectors: N.W., N.E., S.W., and S.E. (Fig. 3).

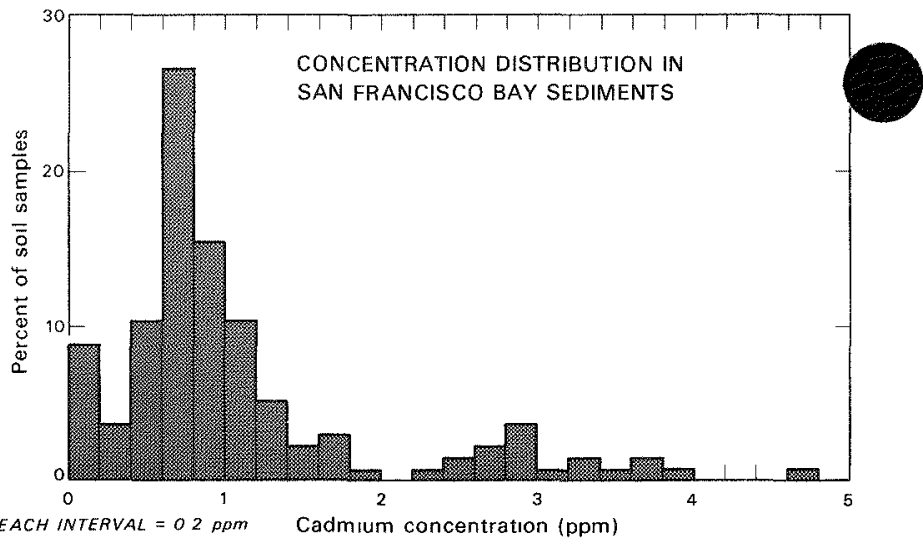

Fig. 1. Histogram distribution of all Bay sediment samples analyzed without regard to core position (top fraction or bottom fraction). (DBL 742-4645)

SAN FRANCISCO BAY PERIMETER

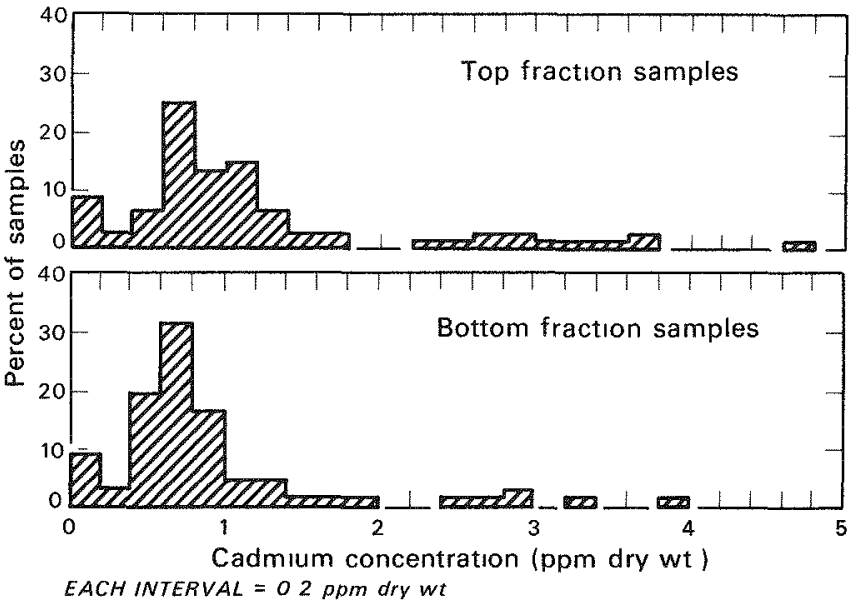

Fig. 2. Separation of Fig. 1 into top and bottom fractions. Plots indicate a more constant Gaussian distribution in the bottom fractions.

(DBL 747-4869)

FOUR SECTOR BREAKDOWN AND DISPLAY OF CADMIUM IN SAN FRANCISCO BAY SEDIMENTS

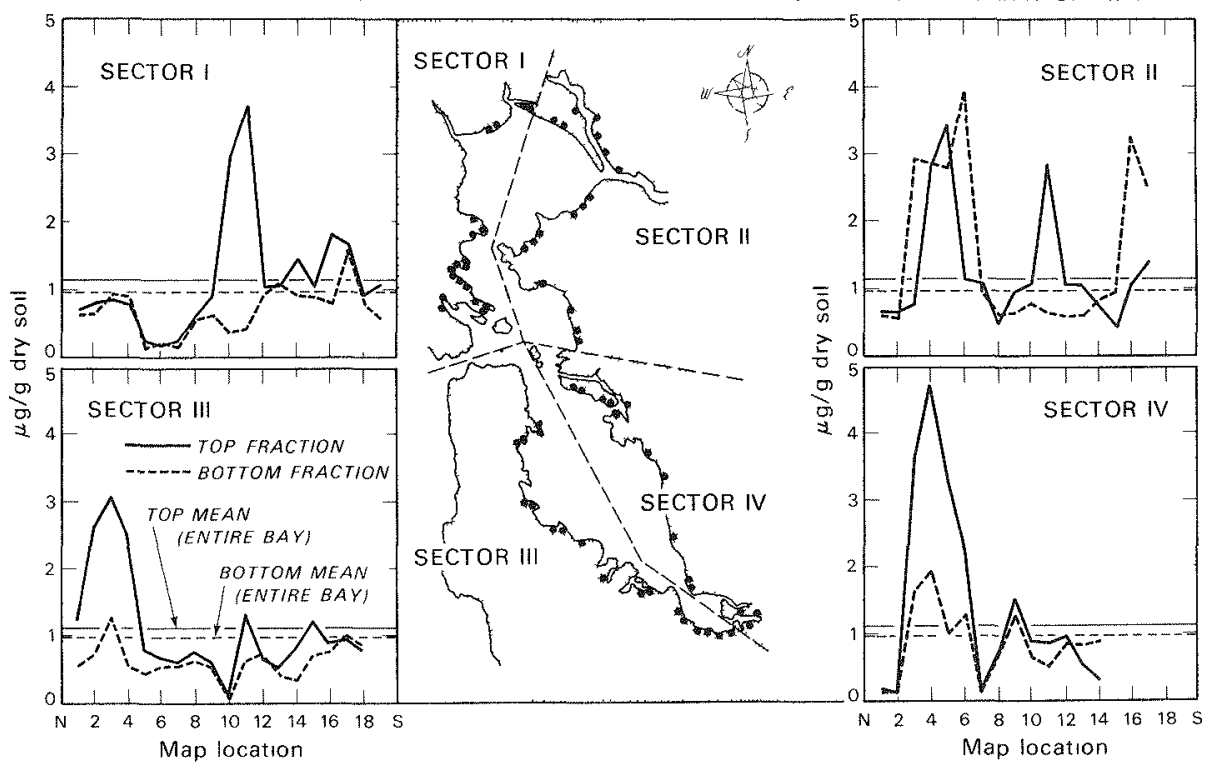

Fig. 3. Four sector breakdown of San Francisco Bay. Curves compare adjacent sites and relationship of each site to top fraction and bottom fraction mean values. Map locations correspond to plotted points by passing from the northernmost sample to the southernmost sample in each sector.

(XBL 742-4649) 
Table 1 . Total bay and sector means with standard deviations

\begin{tabular}{|c|c|c|c|c|}
\hline Sector & $\begin{array}{c}\text { No. } \\
\text { of } \\
\text { Samples }\end{array}$ & Fraction & $\begin{array}{c}\text { Means } \\
\text { and } \\
\text { standard } \\
\text { deviation } \\
\end{array}$ & $\begin{array}{l}\text { Top/Bottom } \\
\text { ratio }\end{array}$ \\
\hline Entire bay (a11 sectors) & $\begin{array}{r}68 \\
68 \\
136\end{array}$ & $\begin{array}{l}\text { Top } \\
\text { Bottom } \\
\text { Combined }\end{array}$ & $\begin{array}{l}1.22 \pm 0.99 \\
0.93 \pm 0.75 \\
1.07 \pm 0.89\end{array}$ & 1.31 \\
\hline Sector I (northwest sector) & $\begin{array}{l}19 \\
19 \\
38\end{array}$ & $\begin{array}{l}\text { Top } \\
\text { Bottom } \\
\text { Bombined }\end{array}$ & $\begin{array}{l}1.14 \pm 0.87 \\
0.70 \pm 0.34 \\
0.92 \pm 0.70\end{array}$ & 1.61 \\
\hline Sector II (northeast sector) & $\begin{array}{l}17 \\
17 \\
34\end{array}$ & $\begin{array}{l}\text { Top } \\
\text { Bottom } \\
\text { Combined }\end{array}$ & $\begin{array}{l}1.25 \pm 0.86 \\
1.54 \pm 1.14 \\
1.40 \pm 1.02\end{array}$ & 0.88 \\
\hline Sector III (southwest sector) & $\begin{array}{l}18 \\
18 \\
36\end{array}$ & $\begin{array}{l}\text { Top } \\
\text { Bottom } \\
\text { Combined }\end{array}$ & $\begin{array}{l}1.10 \pm 0.78 \\
0.65 \pm 0.24 \\
0.88 \pm 0.62\end{array}$ & 1.71 \\
\hline Sector IV (southeast sector) & $\begin{array}{l}14 \\
14 \\
28\end{array}$ & $\begin{array}{l}\text { Top } \\
\text { Bottom } \\
\text { Combined }\end{array}$ & $\begin{array}{l}1.43 \pm 1.41 \\
0.85 \pm 0.53 \\
1.14 \pm 1.10\end{array}$ & 1.66 \\
\hline
\end{tabular}

\section{Footnote and References}

The mean top, bottom, and combined values for the perimeter and sectors of the bay core samples $(n=68)$ are given in Table 1 .

The mean top fraction value for the perimeter of the bay was $1.22 \pm 0.99 \mathrm{ppm} \mathrm{d}$.wt., and the mean bottom fraction value for the perimeter was $0.95 \pm$ $0.75 \mathrm{ppm}$ d.wt. The mean combined fraction value was $1.07 \pm 0.89 \mathrm{ppm} \mathrm{d.wt}$. The top fraction cadmium content was 1.5 to 2.0 times more concentrated than the bottom fraction except in the northeast sector where the fresh water runoff and periodic dredging of the area aids in mixing the surface muds. Due to the proximity of a now closed lead slag fuming plant, petroleum refineries, and extensive shipyard work in the area, the average cadmium content of section II was 1.5 times that of the other sectors. This sector was also the only sector to have bottom fraction values in excess of $2.0 \mathrm{ppm} \mathrm{d.wt.}$
*Condensed from LBL-2642 and from Trace Substances in Environmental Health, VIII, D.D.Hemphi11, ed., U. of Missouri Press, 1974.

1. L. Friberg, M. Piscator and G. Nordberg, Cadmium in the Environment, Chemical Rubber Co., Cleveland, 1971.

2. W. Fulkerson and H. E. Goelker, ed., Cadmium: The Dissipated Element, ORNL-NSF-EP-21, Oak Ridge National Laboratory, 1973.

3. N. Yamagata and I. Shigematsu, Bull. Inst. Publ. Health, Tokyo 19: 1-27, 1970.

4. J. A. Lager and G. Tchobanoglous, J. Sanit. Eng. Div. SA2 (5891): 213-236, 1968.

5. E. S. Pearson, P.N. Storrs and R. E. Selleck, Final Report. A Comprehensive Study of San Francisco Bay ( 8 vols.), SERL, University of California, Berkeley, 1970.

6. L. A. Estvelt, W. J. Kaufman and R. E. Selleck, A study of toxicity and biostimulation in San Francisco Bay-Delta water, State Water Resources Control Board Pub1. 44, Vo1. 4, 1972.

\section{PARTITIONING OF LEAD IN ESTUARINE WATERS OF SAN FRANCISCO BAY}

Don Girvin

This work is directed toward obtaining information on the partitioning of trace metals between the 1iquid and solid (suspended particulate) phases xisting in the water colum and the chemical ehavior of the metals when they are associated with these phases. This information is fundamental to the understanding of estuarine toxic metal transport and toxic metals uptake in marine and estuarine organisms, and is required for an adequate definition of criteria for waste water disposal.
This investigation has examined the partitioning of $\mathrm{Pb}$ and its chemical behavior in the water column of Mare Island Strait of San Francisco Bay during the winter of 1973-74. This work was carried out in conjunction with the LBL study of possible dredge-induced trace metal mobilization and the subsequent accumulation in estuarine organisms. 1 Mare Island Strait is that section of the lower Napa River which provides access to the Navy's nuclear submarine base at Mare Island, Vallejo. The salinity 
of these waters is between 1 and 8 ppt; that of central San Francisco Bay is 25-30 ppt. The Strait is dredged biannually by the Army Corps of Engineers.

Samples were obtained at three stations in the Strait, on six separate occasions (before, during and after dredging), at a depth of 15 feet, during ebb tide in mid-channel. The three sites were in the center of the dredge area, one mile upstream from the dredge area and at the south end of the channel near Carquinez Strait.

From each sample, two subsamples were analyzed (for more details, see Ref. 1). The first subsample of raw water was centrifuged to separate suspended particles $\geqslant 0.6 \mu$ esd $)^{2}$ from supernate. The supernate was drawn off, adjusted to $\mathrm{pH} 2$ with NBS $\times 2$ distilled HC1, heated to $55^{\circ} \mathrm{C}$ for three days, and analyzed for $\mathrm{Pb}$ by anodic stripping, voltametry (ASV) as described by Girvin et al. 3 In the discussion below, the concentrations measured in this fraction are referred to as Phase $1 \mathrm{~Pb}$. They represent that fraction of the $\mathrm{Pb}$ which is in soluble form, plus that $\mathrm{Pb}$ which is $\mathrm{HCl}$ extractable from both inorganic and organic material remaining in the supemate. The solids separated by centrifugation were analyzed by $X$-ray fluorescence. This $\mathrm{Pb}$, associated with suspended particles in the water column will be referred to as Phase $3 \mathrm{~Pb}$. The second subsample of raw water, with all suspended material present, was acidified and heated as above prior to analysis for $\mathrm{Pb}$ by ASB. Lead in this fraction will be referred to as Phase $2 \mathrm{~Pb}$. Phase $2 \mathrm{~Pb}$ concentrations include Phase $1 \mathrm{~Pb}$ plus that $\mathrm{Pb}$ which is $\mathrm{HCl}$ extractable from all suspended particles in the sample.

The results for these analyses are given in Table 1. With one exception, $99 \%$ or more of the $\mathrm{Pb}$ in the water column was associated with suspended particles (Phase 3). This is conservative since smaller particles were not separated from the supernate. Thus, less than 1: of the total. lead in the water was in the solution phase, i.e., dissolved, bound to organics in solution and/or associated with particles $(<0.6 \mu \mathrm{esu})$. One can conclude, first that the most biologically available phase, the solution phase, contains a relatively low fraction of the total $\mathrm{Pb}$ to which organisms are exposed, and second that the major transport mechanism for $\mathrm{Pb}$ in these waters is the movement of suspended particles. It is strongly suspected that other toxic metals exhibit similar partitioning and transport behavior; this is currently being investigated.

The partitioning of $\mathrm{Pb}$ among the various geochemical components of the suspended particles specifies that fraction of the $\mathrm{Pb}$ which may be biologically accessible to organisms ingesting these particles. Engler et a1. ${ }^{4}$ describe trace metal partitioning in benthic sediments and suspended particles as being among, (i) exchangeable states, (ii) carbonates, (iii) sulfides and organics, (iv) hydrous oxide coatings of $\mathrm{Fe}$ and $\mathrm{Mn}$, and $(\mathrm{V})$ alumino silicates and clay minerals. Exchangeable metals and those associated with organics are potentially biologically available. Using Engler's procedures, Battelle Northwest has performed extractions on Mare Island Strait bottom sediments. Their results

Table 1. Concentrations of $\mathrm{Pb}$ in water and suspended particulates. Values given as mean for samples obtained at three stations. The standard deviations about the mean are usually less than thirty percent of the mean.

\begin{tabular}{|c|c|c|c|c|c|c|}
\hline Collection & $\mathrm{I}$ & $I I^{*}$ & $\operatorname{III}^{*}$ & IV & $v^{*}$ & VI \\
\hline $\begin{array}{l}\text { Phase } 1 \\
\text { (supernate) }\end{array}$ & $0.11 \mathrm{ppb}$ & $0.36 \mathrm{ppb}$ & $0.34 \mathrm{ppb}$ & $<0.07 \mathrm{ppb}^{(1)}$ & $<0.07 \mathrm{ppb}^{(1)}$ & -- \\
\hline $\begin{array}{l}\text { Phase } 2 \\
\text { (total acid } \\
\text { extractable) }\end{array}$ & $3.1 \mathrm{ppb}$ & $2.9 \mathrm{ppb}$ & $5.8 \mathrm{ppb}$ & 4.2 ppb & $3.0 \mathrm{ppb}$ & $6.0 \mathrm{ppb}$ \\
\hline \multicolumn{7}{|c|}{$\begin{array}{l}\text { Phase } 3 \\
\text { (tota1 associated } \\
\text { with particulates) }\end{array}$} \\
\hline A. (dry wts.) & $38 \mathrm{ppm}$ & $39 \mathrm{ppm}$ & 57 ppm & $31 \mathrm{ppm}$ & $64 \mathrm{ppm}$ & 39 ppm \\
\hline B. (wet wts.) & $9 \mathrm{ppb}$ & $8 \mathrm{ppb}$ & $26 \mathrm{ppb}$ & $18 \mathrm{ppb}$ & $12 \mathrm{ppb}$ & $25 \mathrm{ppb}$ \\
\hline $\begin{array}{c}\text { Phase } 1 \text { is } X \% \\
\text { of Phase } 3 B\end{array}$ & $1 \%$ & $4 \%$ & $1 \%$ & $1 \%$ & $1 \%$ & - \\
\hline $\begin{array}{c}\text { Phase } 2 \text { is } X \% \\
\text { of Phase } 3 B\end{array}$ & $34 \%$ & $36 \%$ & $22 \%$ & $24 \%$ & $25 \%$ & $24 \%$ \\
\hline
\end{tabular}

*Dredging occured during these collections

(1) Supernate for collection I-III contained particles $<0.6 \mu$ esd, for IV and $V$ this was reduced to $<0.2 \mu$ esd. 
indicated that for surface sediments $46^{\circ}$ of the tal lead was associated with organics and ilfides, $13 \%$ with Fe hydrous oxides, and $41 \%$ bound interstitially in alumino silicates and clay minerals. No measurable lead was present in either the exchangeable states, carbonates or hydrous oxides of Mn. ${ }^{5}$ These bottom sediments exist in a slightly reducing environment.

Suspended particles exist under oxidizing conditions, thus metals as sulfides have been converted to their oxides via oxidation of sulfide to sulfate. Of the total $\mathrm{Pb}$ associated with the suspended particulate samples, $25-35 \%$ is ICCI extractable (Phase 2). A pH solution of HCl does not alter most common aluminosilicate clay structures; ${ }^{6}$ therefore $\mathrm{Pb}$ bound interstitially will not be extractable by our acid treatment. The interpretation of how the $\mathrm{HCl}$ extractable $\mathrm{Pb}$ is partitioned among states i) to iv) listed above is not clear since $\mathrm{HCl}$ will extract all exchangeable $\mathrm{Pb}$ and will dissolve a significant portion of the organic components, carbonates and hydrous oxides of $\mathrm{Mn}$ and $\mathrm{Fe}$. Thus, this work does not establish what portion of total particulate $\mathrm{Pb}$ is in biologically available form but rather provides only an upper limit.

The heaviest periods of rainfall during the winter of 1973-74 coincided with collections III and $V$. Thus the increase in phase 3 (dry wt.) and Phase $1 \mathrm{~Pb}$ cannot be attributed to dredging alone, since urban runoff into Mare Island Strait was a significant factor. 1

It is felt that the increase in both Phase 1 $\mathrm{Pb}$ (collections II and III) and Phase $3 \mathrm{~Pb}$ (III and V) was due to an increase in the relative amount of suspended particulate in the $4 \mu$ to . $1 \mu$ esu range. This resulted from both dredging and rainfa11. The increase in Phase $1 \mathrm{~Pb}$ was not due to an increase in soluble $\mathrm{Pb}$ species but rather to an increase in particulates $\leqslant 0.6 \mu$ esu (not removed by centrifugation). This interpretation is consistent with the fact that (1) increased centrifugation reduced Phase $1 \mathrm{~Pb}$ below ASV detection limits in later samples, and (2) the concentrations of particulate bound $\mathrm{Pb}$ in San Francisco Bay are a factor of 2 greater in the 4-1 $\mu$ range and a factor of 4 greater in the $1.0-0.1 \mu$ range than in suspended particles $>4 \mu .7$ These increases observed in Phases 1 and $3 \mathrm{~Pb}$ at Mare Island may indeed be significant to estuarine organisms feeding on suspended particles $<4 \mu$ esu as the release of metals in soluble form.

\section{References}

1. V. C. Anderlini, J. W. Chapman, D. C. Girvin, A. S. Newton and R. W. Risebrough, "Dredging Impact-Heavy Metals Uptake Study", LBL Report No. UCID-3666 (Sept. 1974).

2. esd = equivalent spherical diameter. In later samples all particles with esd $\geqslant 0.2 \mu$ were separated. Particle sizes are calculated assuming a density of $2.6 \mathrm{~g} / \mathrm{cm}^{3}$.

3. D. C. Girvin, et al., Marine Chemistry 2, 69 (1974).

4. R. M. Engler and J. M. Brannon, "A Practical Selective Extraction Procedure for Sediment Characterization", Am. Chem. Soc. Symp. on Chemistry of Marine Sediments, Atlantic City, N.J., Sept. 1974.

5. Batte11e Northwest Laboratory, "Characterization of Pollutant Availability for San Francisco Bay Dredge Sediments Crystalline Matrix Study", Final Draft Report submitted to U.S.Army Engineer District, San Francisco, Contract No. DACW07-73-C-0080, (1975).

6. S. Ray, H. R. Gault and C. G. Dodd, Am. Minerologist 42, 681 (1957)。

7. D. C. Girvin and A. T. Hodgson, "Trace Element Concentrations Versus Particle Size for San Francisco Bay Seston", in preparation.

\section{DREDGING TMPACT - HEAVY METALS UPTAKE STUDY*}

Victor C. Anderlini, John W. Chapman, Donald G. Girvin, Amos S. Newton and Robert W. Risebrough

The heavy metals uptake study was designed to determine whether the disturbance caused by maintainance dredging of bottom sediments results in the release of heavy metals from the disturbed sediments, and if so released, whether these metals are taken up and accumulated by the invertebrate organisms in the area. The area studied was Mare Island Strait and its vicinity, near Vallejo, California.

A pre-survey of the area showed the presence of some 40 species of benthic invertebrates. Of these benthic species, three species that live in d obtain their food from benthic sediments were osen for study because of their abundance and ide distribution in the area. These species represented three different phyla; Annelida (Polychaeta) Neanthes succinea; Molusca (Bivalvia) - Macoma balthica; and Arthropoda (Crustacea, Amphipoda) Ampelisca milleri. Native populations of the fouling organisms, the cosmopolitan black musse1 Mytilus edulis and the Atlantic ribbed mussel Ischadium demissum (an introduced species) were studied as were populations of Mytilus edulis transplanted from Tomales Bay (a clean area).

Samples were collected from stations shown in Fig. 1 at various times before, during and after each of two dredge periods. Samples of benthic organisms were obtained by scuba divers. Samples for population studies were collected in PVC cores, while samples for metals analysis were obtained with a hydraulic dredge that collected the benthic organisms in a Nylon mesh bag. The samples were washed and purged for three days in "clean" water collected from the same area as the samples. The organisms were then sacrificed and the flesh was collected and digested in dilute tetramethyl ammonium hydroxide for 8 to 10 hours, freeze dried, powdered, and a thin disk prepared for $\mathrm{X}$-ray fluoresence 


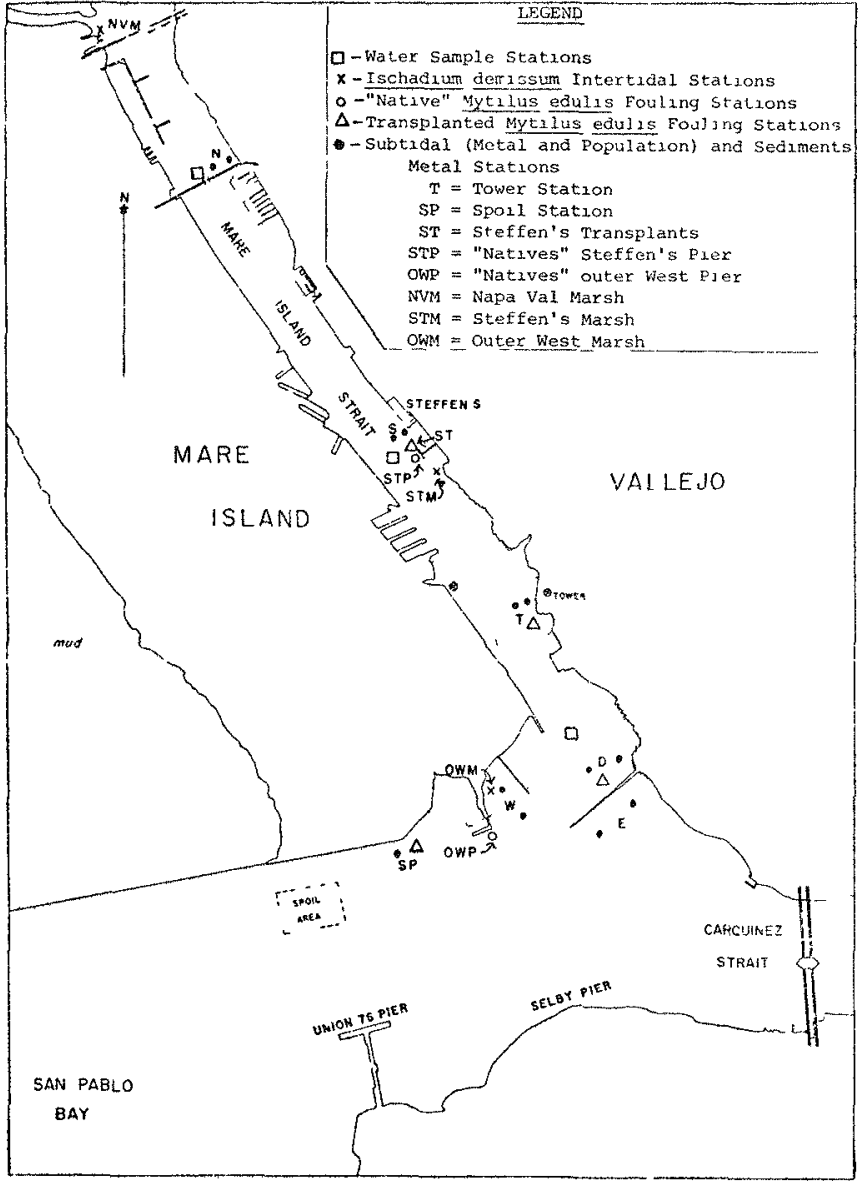

Fig. 1. Sample collection stations in Mare Island Strait and vicinity.

(XBL 746-1035A) analysis. 1 The whole bodies of the amphipod A. milleri, and the polychaete $N$. succinea were digested. The powdered samples were analyzed directly for mercury with the Isotope Shift Zeeman Atomic Absorption apparatus (IZAA) developed at LBL. ${ }^{2}$ Some 10 to 25 individual samples were analyzed to establish the metal content of each respective species at each respective collection station and time. Water samples were analyzed for lead by anodic stripping voltametry.3,4

The following studies were made: 1) The analysis of the heavy metal content of the benthic organisms at various stations near and away from the dredge area and at times before, during and after dredging occurred. 2) The fouling organisms, Mytilus edulis and Ischadium demissum were periodically collected and the flesh analyzed for heavy metals. 3) Some 1200 individual M. edulis were transplanted from Tomales Bay to Mare IsIand Strait and the change in heavy metal content studied as a function of time. 4) M. edulis from Selby Pier (a highly contaminated area at the site of an abandoned lead smelter) were transplanted to Tomales Bay to study the desorption of heavy metals from these organisms. 5) Water and the attendant suspended sediments were studied before, during and after dredging operations. Salinity and rainfal1 data were collected. 6) A laboratory study was made to determine the uptake of heavy metals by the clam, Macoma balthica, at various levels of metal contamination of water and at various salinities.

The experimental results of the benthic study consist of the analysis for each of nine elements of some 2000 samples from 6 to 13 collection stations at 7 time intervals. The average of the analyses of each species is presented in Table 1 . The results and conclusions of the study may be summarized as follows.

Table 1. Average concentration ${ }^{a}$ in ppm of heavy metals in freeze dried invertebrates

\begin{tabular}{|c|c|c|c|c|c|c|c|c|}
\hline \multirow[b]{2}{*}{ Element } & \multirow[b]{2}{*}{ Sediment } & \multirow{2}{*}{$\frac{\text { Ampelisca }}{\text { milleri }}$} & \multirow{2}{*}{$\begin{array}{l}\text { Neanthes } \\
\text { succinea }\end{array}$} & \multirow{2}{*}{$\begin{array}{l}\text { Macoma } \\
\text { balthica }\end{array}$} & \multirow{2}{*}{$\begin{array}{l}\text { Ischadium } \\
\text { demissum }\end{array}$} & \multicolumn{3}{|c|}{ Mytilus edulis } \\
\hline & & & & & & Native & $\begin{array}{c}\text { Tomales } \\
\text { Bay }\end{array}$ & Transplant \\
\hline $\mathrm{Ag}$ & 2.2 & 2.0 & 2.0 & 2.4 & 1.0 & 1.0 & 1.0 & 1.0 \\
\hline As & 12.5 & 4.5 & 5.4 & 10.8 & 7.1 & 8.1 & 6.7 & 7.3 \\
\hline $\mathrm{Cd}$ & 2.2 & 2.3 & 1.9 & 1.3 & 15.2 & 28.3 & 2.8 & 3.8 \\
\hline $\mathrm{Cu}$ & 87 & 60 & 32 & 64 & 21 & 16 & 7.1 & 12 \\
\hline $\mathrm{Ni}$ & 90 & 10 & 11 & 9.3 & 3.3 & 10 & 5.5 & 12 \\
\hline $\mathrm{Pb}$ & 47 & 3.0 & 3.1 & 3.1 & 2.8 & 2.5 & 1.0 & 2.2 \\
\hline $\mathrm{Zn}$ & 149 & 69 & 360 & 526 & 74 & 204 & 137 & 179 \\
\hline $\mathrm{Se}$ & 2.0 & 2.6 & 6.5 & 0.8 & 5.2 & 6.3 & 4.1 & 4.5 \\
\hline $\mathrm{Hg}$ & 0.47 & 0.14 & 0.20 & 0.29 & 0.30 & 0.55 & 0.28 & 0.35 \\
\hline
\end{tabular}

Each value is the average of all stations and collection times.

${ }^{b}$ Reference 6 gives an average of $2.4 \mathrm{ppm}$ Cd for surface sediments in this area. 
With the exception of the metals $\mathrm{Cd}$, Se and $n$, all metals were present in higher concentration in the dried sediment than were present in the dried flesh of the animals living in the sediment. Except for $M$. balthica, selenium was present in higher concentration in the animals than in the sediments with which the organisms were associated. Cadmium was markedly concentrated in mussels. 5 Zinc was concentrated in $M$. balthica and $M$. edulis.

The concentration of heavy metals in sediments in Mare Island Strait did not vary markedly with time or with dredging activity. The metal levels in the invertebrates varied more than did the levels in the associated sediments. Variations of the metals levels in invertebrates were not in correlation with the metal levels in benthic sediments or with those in suspended particulates in the water column. The observed changes in heavy metal concentrations in sediments or in organisms were as great in areas remote from dredging activity as they were in areas near the dredge site.

The largest effect on the metal content of invertebrates was caused by the large change in salinity which occurred with the start of heavy winter rains. The effect of salinity changes was also demonstrated in the laboratory experiment where lowered salinity caused uptake of the metals $\mathrm{Cu}, \mathrm{Pb}$, and $\mathrm{Hg}$, while high salinities caused desorption of these same metals. These increases in heavy metals in the invertebrates of Mare Island Strait which were observed can be largely ascribed to the lowered salinity caused by the heavy rumoff from the heavy rains which occurred. The heaviest rains were coincident with the two dredge periods.

The amphipod Ampelisca milleri disappeared from the study area soon after the first dredge period and the first heavy rains, possibly due to migration to areas of higher salinity. The native and the first transplant colonies of $\mathrm{M}$. edulis died at the end of November. An oil spill at Mare Island is believed to be the direct cause.

The transplanted $M$. edulis rapidly approached the same levels of heavy metal contamination as the native $M$. edulis in the same area. The reverse transplānts from Selby Pier to Tomales Bay desorbed heavy metals to approach the levels of the native Tomales Bay mussels. Some of the change was due to salinity changes, but most of the change must illustrate the magnitude of the heavy metal contamination at Mare Island Strait and its environs.

This study gave no evidence that dredging had any direct effect on the heavy metal contamination of the invertebrates in the area.

\section{Footnotes and References}

* Condensed from report UCTD-3666, Sept. 1974. Research supported by the U. S. Army Corps of Engineers.

1. R. D. Giauque, F. S. Goulding, J. M. Jaklevic, and R. H. Pehl, Anal. Chem., 45, 671 (1973).

2. D. A. Church, T. Hadeishi, L, Leong, R. D. McLaughlin, and B. D. Zak, Anal. Chem., 46, 1362 (1974).

3. R. Clem, G. Litton and L. Omelas, Anal. Chem., 45, 1306 (1973) ; R. Clem, M.P.E. Application Notes, 8 (1), 1-8 (1973).

4. C. Pattersen, and D. Sett1e, I.D.OE Study Report No. 2, California Institute of Technology, Pasadena, Califomia 1972.

5. R. A. Horne, "Marine Chemistry", Wiley-Interscience, New York, 1969, p. 155.

6. B. R. Moyer, and T. F. Budinger, LBL-2642, April 1974.

\section{EFFECT OF POLLUTANTS ON MEMBRANE STRUCTURE AND FUNCTION IN MAMMALIAN CELLS *}

L. Packer, R. Mehlhom and J. R. Smith

\section{INTRODUCTION}

In this program we are attempting to characterize immediate and long term effects of pollutants on cells with a view to gaining critical insight into the general mechanisms of action of these compounds on humans and other higher animals. We believe that the primary route by which air pollutants attack the integrity of cell membranes is through oxidative damage. This may occur by introduction of exogenous free radicals into the cel1, by disruption of the pathways by which the cell nomally sequesters endogenous free radicals from sensitive cellular components or by interference with the mechanisms by which the cell normally repairs oxidative damage. The oal of our program is to achieve an understanding of the mechanisms by which environmental agents impair the structure and function of cell membranes, and the defenses by which the cell normally strives to maintain integrity against enviromental agents.
In our initial work a major effort has been devoted to a study of the effects of the naturally occuring anti-oxidant d1- $\alpha$-tocopherol (vitamin $\mathrm{E}$ ) on the lifespan of cells in culture. In addition, ionic detergents have been used to study the correlation between structural disruption of submitochondrial membranes and the loss of their energy transducing functions. This latter effort has been complimented by work on the development of sensitive methods for the analysis of membrane structure.

\section{EXTENSION OF THE LIFESPAN OF HUMAN CELLS IN CULTURE BY VITAMIN $E^{* 1}$}

The finite lifespan of human diploid cells in culture has attracted attention since the proposal by Hayflick and Moorhead ${ }^{2}$ that such a system may serve as a model for cellular aging. For example, morphological and biochemical changes have been observed to occur during the Iifespan 
of these cells in culture, and electron microscopic examination has revealed an increase in the number of 1ysosomes, electron dense residual bodies surrounded by membranes, and amorphous insoluble deposits. A1so, Deamer, 3 using fluorescence microscopy, has demonstrated large increases in diffuse and particulate fluorescent material in cultures near the end of their in vitro 1ifespan. Evidence invoked to support the hypothesis that cell death in this system is relevant to aging in vivo has come from studies showing an inverse correlation between the age of the donor of skin fibroblasts and the lifespan of these cells in vitro. 4 However, it is not known yet whether the limited 1ifespan of these cells in culture is a genetically programmed or environmenta11y induced phenomenon or some combination of both processes.

WI-38 human lung ce11s contain extractable amino iminopropene substances, known to be formed as a consequence of oxidative damage 5 processes that lead to crosslinking reactions. In this respect, Harman's proposal of several years ago, that free radical-mediated reactions might contribute significantly to the aging process, 6 is of interest, particularly in light of recent evidence that anti-oxidants, known to inhibit free radical-mediated reactions, effectively increase the 1ifespan of organisms in vivo?, 8 Antioxidants also inhibit the accumulation of 1ipofuscin (age pigment) in various tissues. 5 Nevertheless, convincing evidence that free radicalmediated reactions are important in 1 imiting the lifespan of human cells has not yet been reported. On this basis, we have chosen to study the effect of vitamin E, a universal, natural antioxidant, on cultured WI-38 embryonic human lung cells. Compared to other systems used to investigate aging processes, the human diploid cell system is relatively simple, and its immediate cellular environment is easily modified. Vitamin E presumably acts to teminate peroxidation reactions in cellular membranes, and one of its basic biological functions seems to be to terminate free radical reactions.

\section{Cell Proliferation}

The effect of $\mathrm{d} 1-\alpha$-tocopherol on the longterm proliferation of WI-38 cells in culture is shown in Fig. 1. In parallel subcultivation series (which originated from a single starter culture) in which two different concentrations of tocopherol were employed $(100 \mu \mathrm{g} / \mathrm{m} 1$ and $10 \mu \mathrm{g} / \mathrm{ml})$ in the medium, it was observed that the cells grown in the presence of tocopherol from the 45 th population doubling level (PDL) consistently had a longer lifespan in vitro than the contro1 cells. The control cells reached Phase III at the 65th population doubling while the treated cells were apparently healthy and were still capable of growing in culture despite the fact that they had been subcultivated for more than 100 population doublings.

After the cultures grown in the presence of 10 and $100 \mu \mathrm{g}$ tocopherol per ml medium had reached the 73 rd population doubling level, tocopherol was removed from the medium and the cells were grown in tocopherol-free medium. These cells

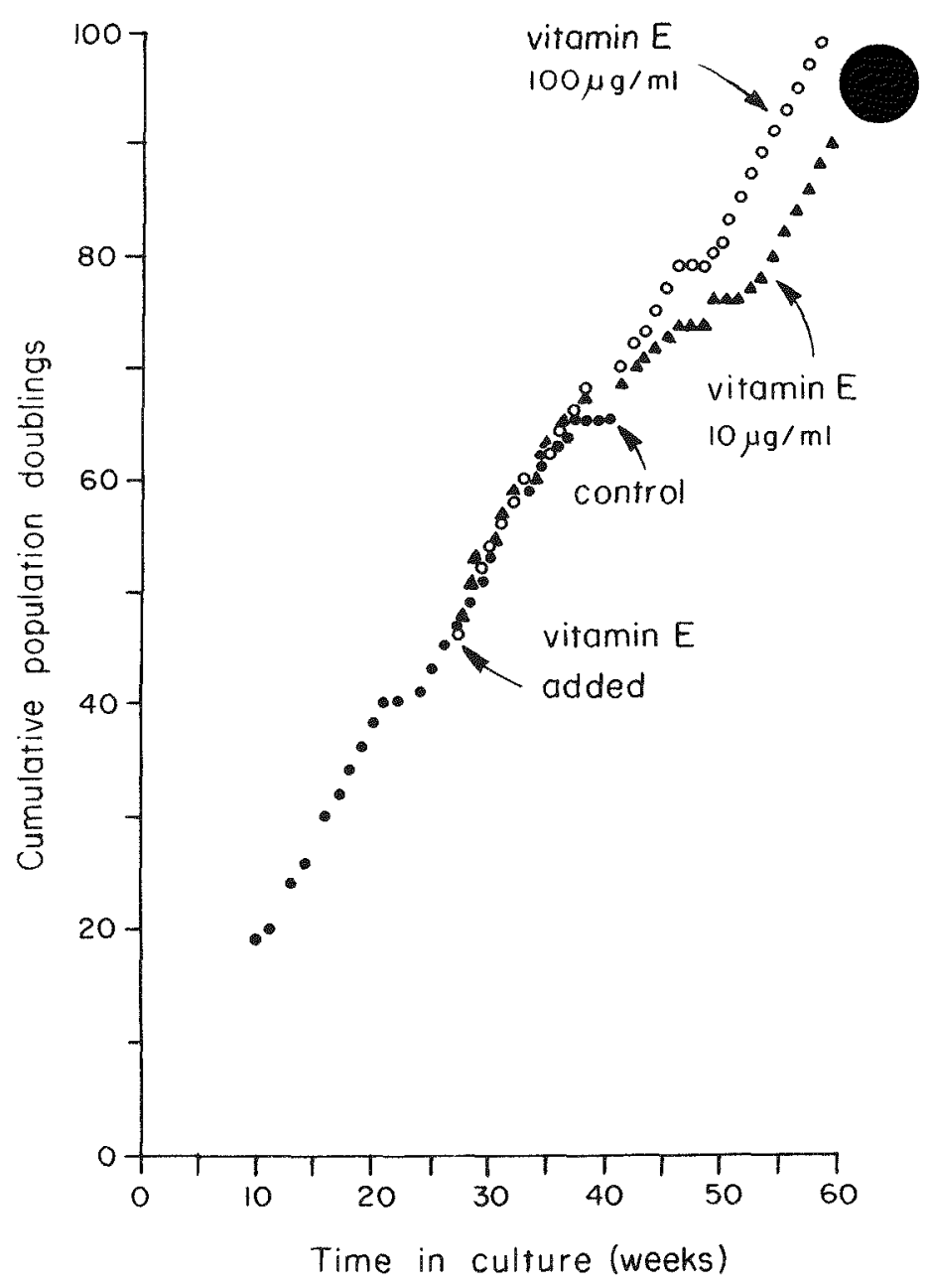

Fig. 1. Effectof d1- $\alpha$-tocopherol on in vitro proliferation of WI-38 human diploid lung fibroblasts. A. Cumulative population doublings in the continuous presence of vitamin $\mathrm{E}$. Conr trol cultures, ; cultures treated continuously from the 45th population doubling level with 10 , or $100 \mu \mathrm{g}$ tocopherol/ml medium, o.

(XBL 748-1408)

have undergone 95 to 100 population doublings and are growing as well as those which have been continuously cultured in tocopherol medium since the 45 th population doubling level. Thus, a relatively brief period of growth in tocopherol appears to have a long-term effect on cell proliferation.

From autoradiographic analysis, performed at every second population doubling from population doubling leve1 85 to 97 on cells continuously exposed to tocopherol, we consistently found that $95 \pm 3 \%$ of the cells were capable of synthesizing DNA between the 24th and 54th hour after subcultivation.

The proportion of cells that attached to the growth surface at subcultivation was determined by trypsinizing and counting the cell suspension with a Coulter counter for control cells and for cells grown in $100 \mu \mathrm{g}$ tocopherol per $\mathrm{ml}$ 


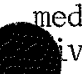
dium at 8 hrs after planting for four consecuve $1: 4$ subcultivations. About $40 \%$ of the concol cells attached, while about $30 \%$ of the tocopherol cells attached at each subcultivation, indicating that the increased number of population doublings observed in tocopherol-treated cultures is due to an increased number of cell divisions rather than a decrease in cell loss upon subcultivation.

The growth of cells at the 20th population doubling in various concentrations of tocopherol is shown in Fig. 2. No effect on growth rate or saturation density was observed at the concentrations of tocopherol used in this experiment. However, an increase in the time between subcultivation and the beginning of cell division was observed with 100 and $300 \mu \mathrm{g}$ tocopherol/m1 of medium. Hence at the concentrations of tocopherol used for long term growth experiments, little effect on the short term growth of WI-38 ce11s occurs.

\section{Normalcy of WI-38 Cells with Increased Lifespan}

Tocopherol treated cells were subjected to a number of tests after the 85 th population doubling level to detemine whether they met criteria for normal human cells. Cells which had been continuously treated with $100 \mu \mathrm{g}$ tocopherol per ml medium exhibited density dependent inhibition of proliferation expected for normal WI-38 cells as shown by examining growth curves of tocopherol-treated cells at the 93rd population doubling level and control cells at the 25th population doubling level.

Tocopherol-treated cells were subjected to karyotypic analysis at the 85 th and 100 th population doubling level by direct microscopic analysis of chromosome preparations. In addition, the 100th population doubling level culture was subjected to more stringent analysis, requiring photographic reconstruction of $20 \%$ of the chromosome "spreads" examined. Table I summarizes the karyotypic data from these two analyses. Both cultures had a normal human diploid karyotype.

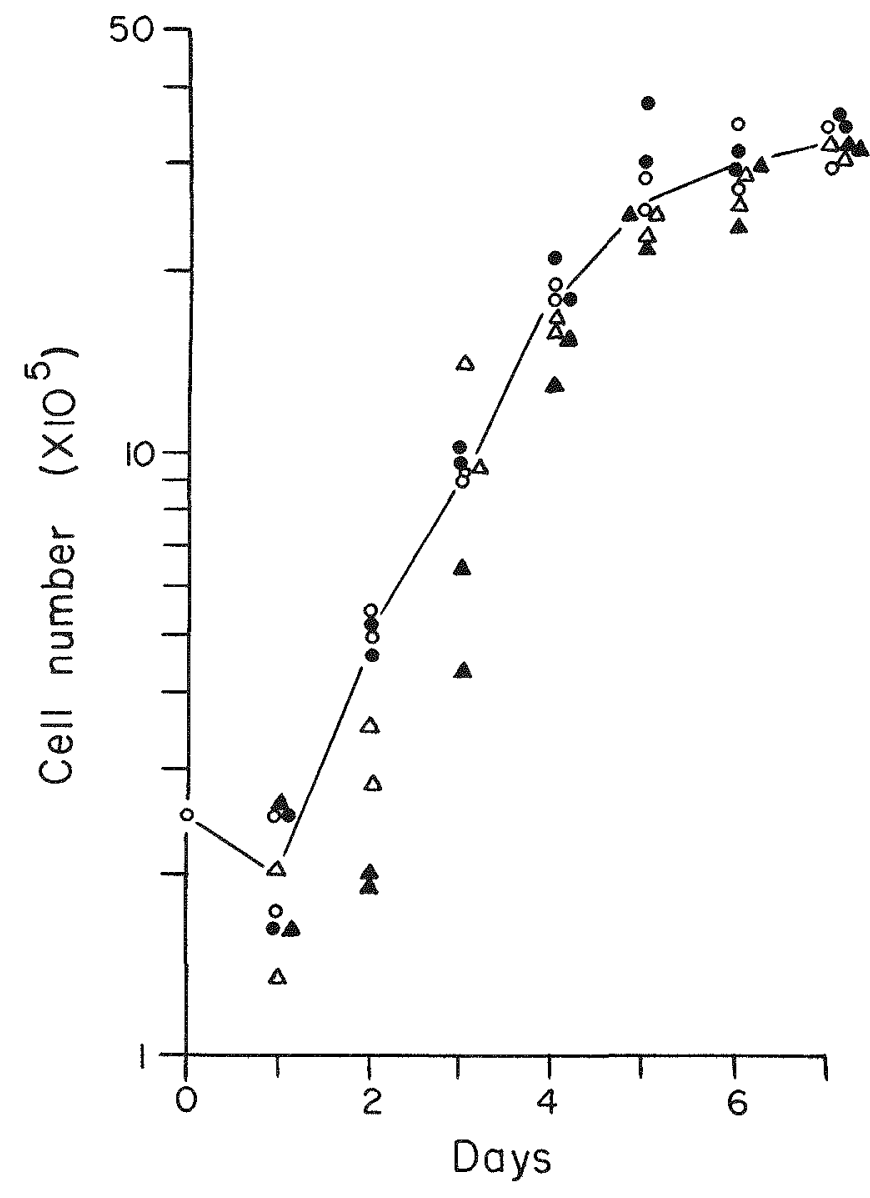

Fig. 2. Growth of cells at the 20th population doubling level in various concentrations of d1- $\alpha$ - tocopherol. $2.5 \times 10^{5}$ cells $/ 25 \mathrm{~cm}^{2}$ flask were planted on day zero. Duplicate flasks at each concentration were counted each day. The medium was not changed during this experiment. $-\mathrm{o}$, contro $1 ;-, 10 \mu \mathrm{g} / \mathrm{m} 1$; $\triangle-, 100 \mu \mathrm{g} / \mathrm{ml} ;-\mathbb{A}, 300 \mu \mathrm{g} / \mathrm{ml}$.

(XBL 748-1409)

Table 1. Karyotypic analysis of WI-38 cells

\begin{tabular}{|c|c|c|c|c|c|c|c|}
\hline \multirow[b]{2}{*}{ Cultures } & \multicolumn{7}{|c|}{ Frequency (cells per 100) } \\
\hline & $\begin{array}{l}\text { Hyper- } \\
\text { diploid }\end{array}$ & diploid & Polyploidy & $\begin{array}{c}\text { Chromosome } \\
\text { breaks }\end{array}$ & $\begin{array}{c}\text { Chromatid } \\
\text { breaks }\end{array}$ & $\begin{array}{l}\text { Structural } \\
\text { abnormalities }\end{array}$ & $\begin{array}{c}\text { Other } \\
\text { abnormalities }\end{array}$ \\
\hline PDL 85 & 0 & 6 & 0 & 0 & 5 & 2 & 0 \\
\hline PDL 100 & 1 & 16 & 0 & 1 & 1 & 1 & 0 \\
\hline $\begin{array}{l}\text { Maximum acceptable } \\
\text { number }\end{array}$ & 2 & 18 & 4 & 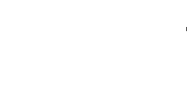 & & 2 & $\begin{array}{l}\text { At discretion } \\
\text { of control } \\
\text { authority }\end{array}$ \\
\hline
\end{tabular}

PDL = population doubling level. 
Ce1ls at the 100th population doubling level met all the exacting criteria for normalcy set down by the 1969 and 1971 meetings of the Committee on Cel1 Culture.

Cells from the same subcultivation series were tested for the presence of SV-40 tumor antigen (SV-40 T-antigen) and showed no indication of SV-40 transformation according to this criterion. When tested in paralle1 with the tocopherol-treated WI -38 cells, cells known to be transformed by SV -40 gave positive $\mathrm{T}$-antigen reactions.

Examination of tocopherol-treated cells by light microscopy after more than 90 population doublings revealed that these cells manifested the same properties as young cells in terms of size, shape and pattern of growth.

Although the role of tocopherol in this series of experiments showing a longer lifespan is unexplained it seems clear that the cells which survired are apparently normal by the above criteria.

\section{Incorporation of Tocopherol into the Subce1lular Fraction}

To determine whether tocopherol added to the medium was incorporated into cellular organelles, we followed the fate of tritium-labeled tocopherol mixed with unlabeled tocophero1. About $85 \%$ of the tocopherol was lost from the medium because of adsorption to the sides of the flask and formation of a thin film of tocopherol on top of the medium within the first $24 \mathrm{hr}$. When $100 \mathrm{\mu g}$ of tocopherol was added per $\mathrm{ml}$ of medium, about $0.5 \%$ of the tocopherol remaining in the medium was encorporated into cellular organelles $(100,000 \mathrm{x}$ pellet after sonication). This is equivalent to $2-3 \mu \mathrm{g}$ tocopherol per mg cellular protein.

\section{Protection Aga inst Oxidative Damage by Tocophero1}

If the mechanism of $d 1-\alpha$ - tocopherol in prolonging cellular lifespan is due to its action as an antioxidant, it should prevent accumulation of oxidative damage to medium constituents or to cellular components. To test this, WI-38 cells were examined for the presence of thiobarbituric acid reactive material, indicative of the formation of malondialdehyde, a hydroperoxide deomposition produce of polyunsaturated fatty acids. In a typical experiment, control cells showed 0.7 to 1.0 and tocopherol treated cells 0.08 to 0.5 nmoles of thiobarbituric acid reactive material per $\mathrm{mg}$ protein. Examination of cells grown in the presence of tocopherol by fluorescence microscopy revealed the virtual absence of the fluorescent material shown by Deamer ${ }^{3}$ to be associated with non-dividing cells.

Protection by d1- $\alpha$-Tocopherol Against Environmental Stress

To further evaluate the potential protective action of $d 1-\alpha$-tocopherol on $W T-38$ cell proliferation, experiments were designed in which the cells were subjected to acute environmental stress by raising the oxygen tension and by illuminating with visible light. It is generally agreed that such environmental stress leads to free radical produc tion and subsequent oxidative damage by peroxidation.

Treatment of WI-38 cells with visible 1 ight decreased the number of viable cells in cultures without tocopherol by $90 \pm 5 \%$ compared to unirradiated cultures. When $1 \mathrm{mg}$ tocopherol per $\mathrm{ml}$ medium was present in the balanced salt solution, the decrease in the number of viable cells was only $30 \pm 10 \%$. The number of viable cells in control cultures was not decreased by standing at room temperature in Hanks' balanced salt solution $+5 \%$ fotal calf serum for 20 hrs.

In other experiments, we observed about $90 \%$ inhibition of division rate when cultures were place placed in an atmosphere composed of $40 \%$ oxygen, $55 \%$ nitrogen and $5 \%$ carbon dioxide as compared to control cultures grown in $95 \%$ air and $5 \%$ carbon dioxide. oxide. The addition of $1 \mathrm{mg}$ of tocopherol per $\mathrm{ml}$ of medium resulted in a partial reversal of this inhibition to about $50 \%$ of the valuc obtained in $20 \%$ oxygen without added tocopherol. No cell growth was obtained in an atmosphere of $60_{0}^{\circ}$ oxygen.

The experiments reported here suggest that in an appropriate environment, human cells may be capable of a very large number of divisions, and perhaps an indefinite number. It is generally accepted that tocopherol exerts its protective action by inhibiting oxidative damage. Tocopherol is preferentially partitioned into the hydrophobic areas of membranes where this substance comes in close proximity to the unsaturated fatty chains of 1ipids to stabilize the membrane. The environment in which human cells are usually cultured may contain inadequate quantities of natural antioxidants. The effectiveness of vitamin $E$ as an antioxidant in this sys tem is indicated by lower amounts of detectable thiobarbituric acid reactants and protection from the deleterious effects of high oxygen concentration and photosensitized cellular damage.

The action of $\mathrm{d} \mathrm{l}-\alpha$-tocopherol in prolonging the 1 ifespan suggests that free radical-mediated peroxidation reactions could damage macromolecules, such as proteins and nucleic acids. We have found evidence that the oxidative capacity of the mitochondria of WI-38 cells is about twice that needed to maintain cell proliferation. 9 In vitamin $\mathrm{E}$ deficiency, WI-38 membranes, particularly those of mitochondria, are readily susceptible to peroxidative attack. The spreading of this process in the cytoplasm could interfere with the turnover of cellular materials resulting in an accumulation of indigestible polymers and defective enzymes. Consistent with such views is the diminished accumulation of fluorescence damage products in vitamin $\mathrm{E}$ treated cells.

This investigation suggests that senescence in WI -38 cells is associated with oxidative damage occurring during their proliferation in culture. A balance probably exists between the occurrence of environmentally induced oxidative damage to cells in culture and their ability to neutralize it. It is possible that by decreasing the rate of occurrence of this damage, its gradual accumulation may be prevented, thus allowing prolonged cellular proliferation. Since tocopherol confers upon 
WI-38 cells a long-term ability to proliferate in ulture, these WI-38 cells provide a unique model bystem for evaluating environmental effects on the growth potential of human cells.

\section{DETERGENT INACTIVATION AND REACTIVATION OF MITO- CHONDRIAL RESPIRATION}

Detergent treatment has been used to study the degree of membrane dismuption that can be tolerated by a cell or subcellular organelle without appreciable loss of biochemical functions. We have used the ionic detergents sodium dodecyl sulfate (SDS, anionic) and cetyl trimethyl ammonium bromide (CTAB, cationic) to follow the loss of mitochondrial respiration resulting from the progressive disruption of sonicated inner mitochondrial membranes isolated from rat livers. The effect of these detergents on the structure of mitochondrial membranes was followed with lipid spin labels.

Figures 3 and 4 show the effect of SDS and CTAB on oxygen consumption of submitochondrial particles (SMP's) using succinate as the substrate for respiration. SMP's are membrane bounded vesicles with ittle trapped protein so the protein concentrations given in the figures refer to membrane proteins. Mitochondrial membranes are composed of about $60 \%$ protein and $40 \%$ lipid. 10 Both detergents inhibit respiration at levels corresponding to about one mole of detergent per mole mitochondrial 1ipid. Similar results were obtained when NADH and ascorbate-TMPD were used as substrates for respiration.

Two spin probes, A12NS and CDTAB, were used to assay membrane disruption. The stearic acid derivative, A12NS probes the hydrophobic membrane interior, while the CTAB analogue, CDTAB, reports

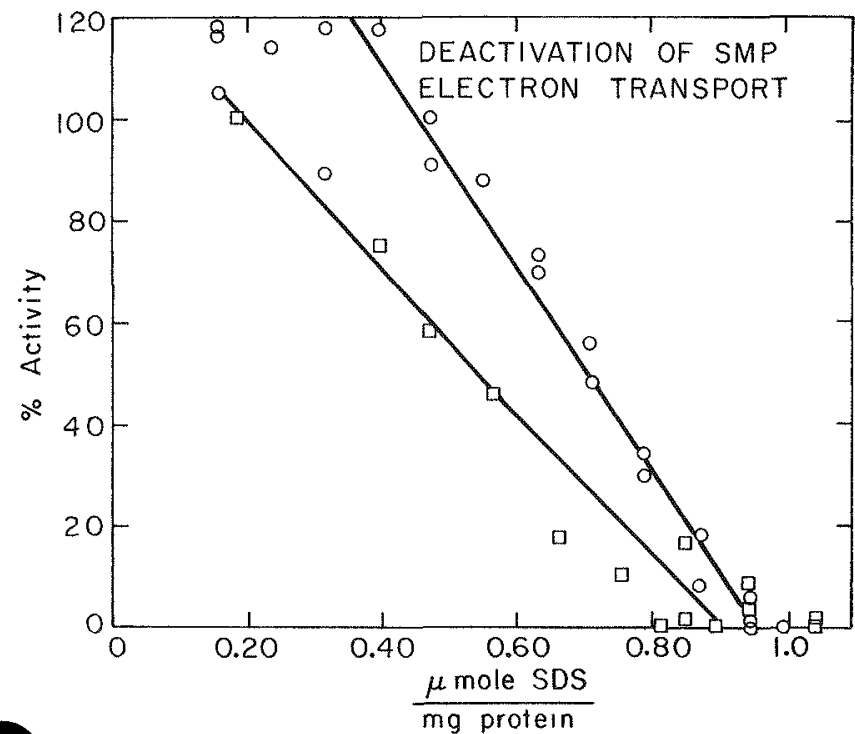

g. 3. Loss of respiratory activity of submitochondrial particles with SDS, substrate: succinate. Circles and squares refer to two different preparations. The protein concentration in the curvette was $1 \mathrm{mg} / \mathrm{ml}$.

(XBL 747-3744)

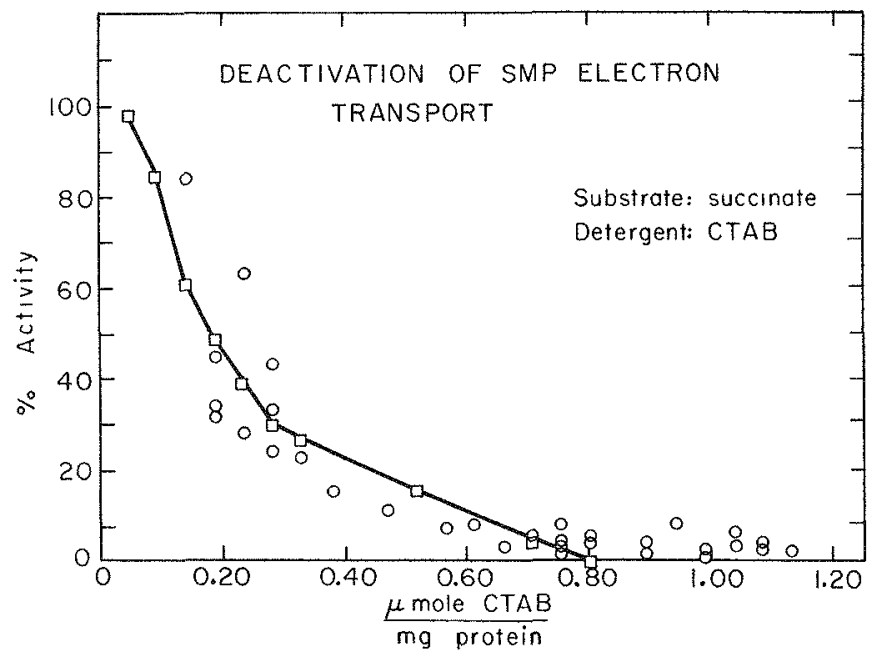

Fig. 4. The effect of CTAB on mitochondrial respiration. Squares refer to one representative experiment while circles refer to three separate preparations.

(XBL 747-3745)

on the aqueous interface of the membrane. The rotational mobility of the nitroxide group (the spin label in A12NS and CDTAB), described by the parameter $\tau_{\text {c (correlation time), was used as a }}$ measure of nembrane fluidity. Results of this study are presented in Figs. 5 and 6 . These EPR studies were carried out with much more concentrated suspensions of SMP's than the respiratory activities,

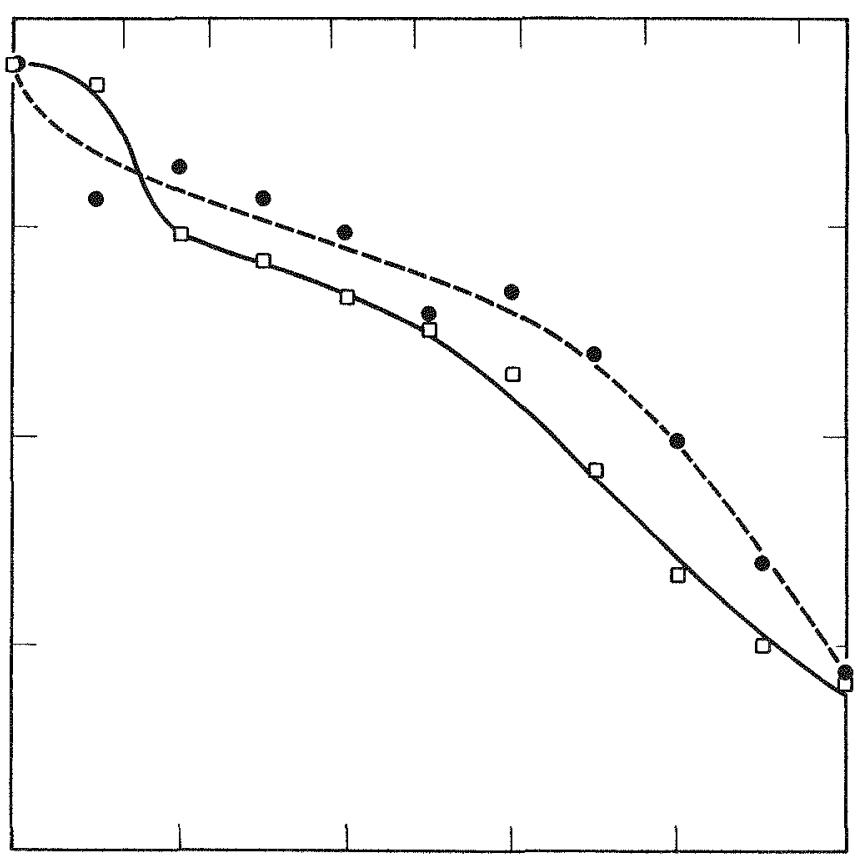

Fig. 5. The effect of the two charged detergents on the rotational mobility of a lipid spin label - A12NS. Different concentrations were obtained by mixing a spin labeled solution of $50 \mathrm{mM}$ of detergent (corresponding to $15 \mathrm{mg} / \mathrm{ml}$ of SDS), with a spin labeled solution of submitochondrial particles at a protein concentration of $15 \mathrm{mg} / \mathrm{m} 1$. (XBL 751-4625) 


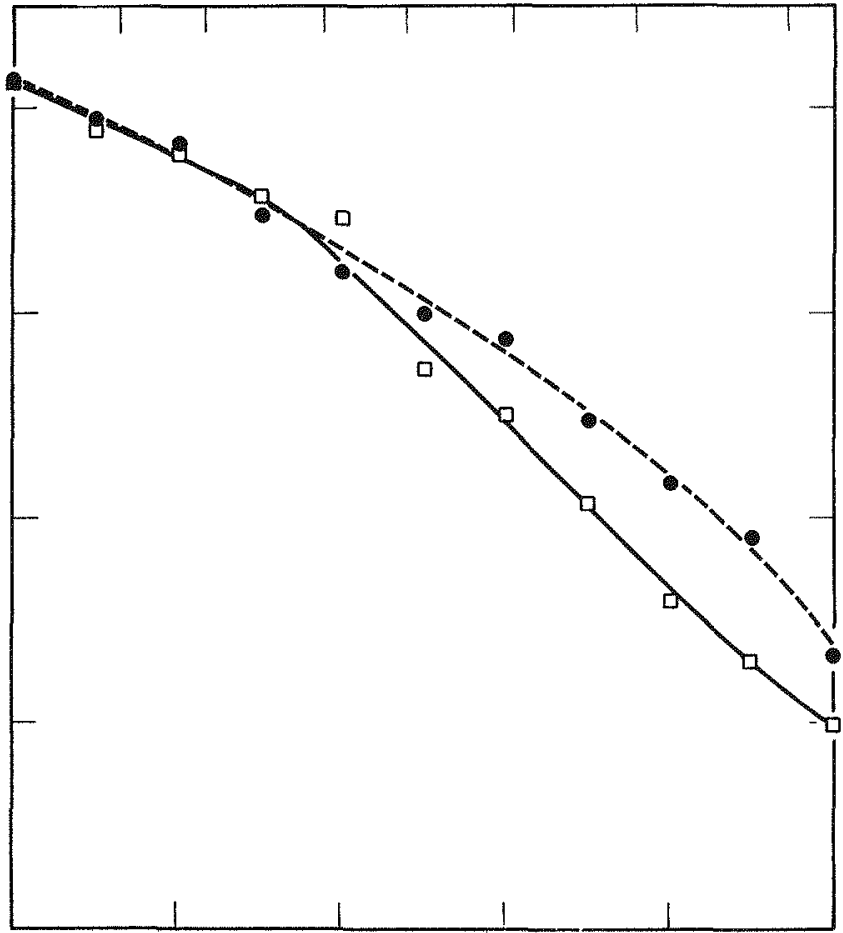

Fig. 6. The mobility of a spin labeled detergent molecule, CDTAB, as a function of SDS or CTAB additions to submitochondrial particles. The mixing procedure was described for Fig. 5.

(XBL, 751-4624) and hence, the detergent concentrations in the membranes cannot be compared directly. However, the concentration of detergent bound to the membranes will be higher in the spin-1abel experiments than in the respiration studies, so it can be concluded that the electron transport activity of SMP's is lost upon very slight loss of structural integrity of the membrane.

The loss of function induced by one ionic detergent is reversible by the addition of a counterion detergent (e.g., the effect of an anionic detergent is reversed by the addition of a cationic detergent.) Optimal reactivation is achieved with about a $30 \%$ excess of SDS over CTAB. Results of a reactivation study of the oxidation of succinate by SMP's are given in Table 2. However, at higher levels of the inactivating detergent no reactivation is possible by charge neutralization. This suggests that the major effect of these detergents in inhibiting electron transport can be ascribed to charge effects at the membrane surfaces and there is a residual disordering effect upon neutralization of excess charge.

The structural similarity of CTAB and its spin labeled analogue, CITAB, was exploited to show that there is an appreciable population of detergent molecules in solution as monomers under the conditions of the respiration experiments. The addition of SDS to the suspension removed these monomers from solution.

Physical studies of pure detergent solutions, including $x$-ray diffraction, spin labeling,

Table 2. Detergent inactivation and reactivation of succinate oxidation by submitochondrial particles. Numbers in parentheses denote the number of experiments which were averaged to arrive at the quoted minimum activities. Due to technical difficulties we could assign only a lower limit to the maximum reconstituted activity in one of the experiments and, hence, the ratio of SDS to CTAB could not be determined.

The designation SDS $\rightarrow$ CTAB means inactivation was carried out with SDS - then reactivation was implemented with CTAB.

\begin{tabular}{|c|c|c|c|}
\hline & \multicolumn{3}{|c|}{ Succinate } \\
\hline & $\begin{array}{l}\text { Minimum } \\
\text { activity } \\
(\%)\end{array}$ & $\begin{array}{l}\text { Reconstituted } \\
\text { activity } \\
\left(\frac{0}{0}\right)\end{array}$ & $\frac{\text { mo1 SDS }}{\text { mol CTAB }}$ \\
\hline \multirow{3}{*}{$\begin{array}{l}\text { SDS } \\
\rightarrow \\
\text { CTAB }\end{array}$} & 48 & 146 & \\
\hline & 17 & 117 & 1.25 \\
\hline & $\begin{array}{c}1.9 \\
\pm 3.3(3)\end{array}$ & $\begin{array}{r}92 \\
\pm 20\end{array}$ & $\begin{array}{l}1.38 \\
\pm .17\end{array}$ \\
\hline \multirow{3}{*}{$\begin{array}{l}\mathrm{CTAB} \\
\overrightarrow{\mathrm{SDS}}\end{array}$} & $\begin{array}{l}40 \\
\pm 6(3)\end{array}$ & $\begin{array}{r}179 \\
\pm 37\end{array}$ & $\begin{array}{l}1.56 \\
\pm .10\end{array}$ \\
\hline & 13 & 233 & 1.20 \\
\hline & $\begin{array}{c}2.5 \\
\pm 1.7(6)\end{array}$ & $\begin{array}{l}150 \\
\pm 22\end{array}$ & $\begin{array}{l}1.41 \\
\pm .14\end{array}$ \\
\hline
\end{tabular}


and centrifugation experiments have demonstrated hat the uncharged equimolar mixture of SDS and $T A B$ has a well ordered crystalline structure with only a limited capacity for solubilizing lipids. These results suggest that reactivation is partially due to the removal of detergents from the membrane by lateral diffusion attended by crystallization of the binary mixture. In this model, crystalline detergent domains will be contiguous with the lipo-protein bilayer arrays of the membrane. Spin labeling experiments are in progress to assess this hypothesis.

\section{REDUCTION OF ELECTRON MICROGRAPHS OF BIOLOGICAL. MEMBRANES}

The freeze fracture technique provides a high resolution view of the interior and surfaces of biological membranes. Most membranes are studded with particles, presumed to be protein, ranging from a diameter of $180 \AA$ downwards. These particles usually seem to be randomly arranged, but occasionally they are observed to be clustered under defined physiological conditions. More subtle clustering may be a general phenomenon, but might have escaped detection by subjective criteria. We have therefore developed a pattern analys is procedure for membrane particles which provides an objective, semi-automatic means of data reduction for electron micrographs of freeze fracture replicas.11

Conversion of the electron micrographs to Euclidean coordinates of the particles is achieved by means of a scanning machine originally designed for the data reduction of bubble chambor films. Many of the particles are at the margin of detection because of size and contrast variations within the replica. Thus fully automatic data reduction had to be rejected in favor of a semiautomatic approach in which a trained observer identifies particles within a designated area of the membrane. Figure 7 gives an example of the conversion of an electron micrograph to computer printout.

Pattern analysis is carried out by means of a computer program written for the CDC 7600 machine. This program subdivides the picture area into sma11. squares and sums up the number of particles in these subdivision. Frequency histograms are compiled as a function of the number of particles per subdivision. Thus a given particle pattern is reduced to a histogram of the number of squares containing a given number of particles versus the number of particles per square. These histograms are then nomalized with respect to the total number of particles in the picture area to facilitate a comparison of sparse and dense patterns.

Random number generators were used to produce reference frequency histograms corresponding to various sets of point particles. These were averaged to provide a reference function which is subtracted from all the membrane particle histograms.

A series of histograms is generated for each picture area covering a range of subdivision
Dark
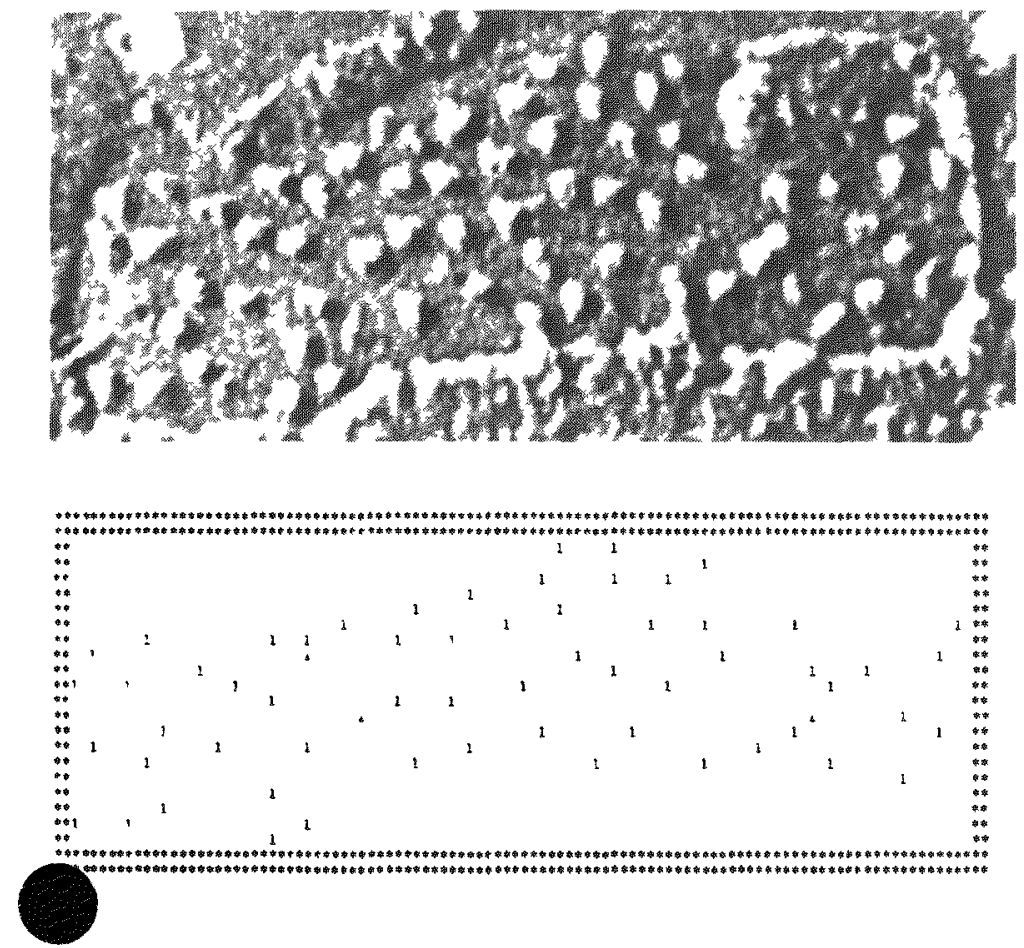

Light
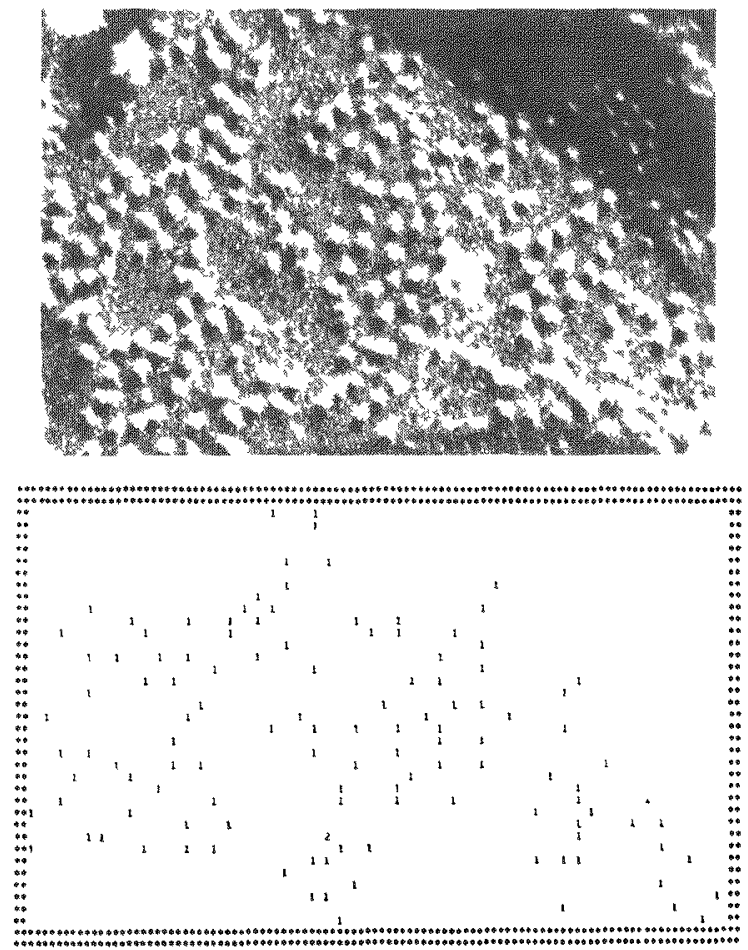

Fig. 7. Examples of the translation of particle positions into computer data. The electron micrographs represent chloroplast membranes which were quickly frozen under dark and illuminated conditions. 12

(XBL 748-5518) 
areas. Each histogram is characterized in terms of a single parameter the second moment about the mean, which is a measure of the width of the histogram. In general the second moment is expected to be larger for a clustered pattern than for a random patterns so the subtraction of random pattern histograms from clustered patterns will lead to a positive difference function. This difference function, designated by " $\Delta$ Particle Frequency" will be a maximm for the subdivision area corresponding to the cluster area.

The results of an analysis of a series of replicas of freeze fractured red cell membranes is given in Fig. 8. Replicas 1, 2, and 3 are judged to be random by subjective criteria. There are deviations from the reference histogram,

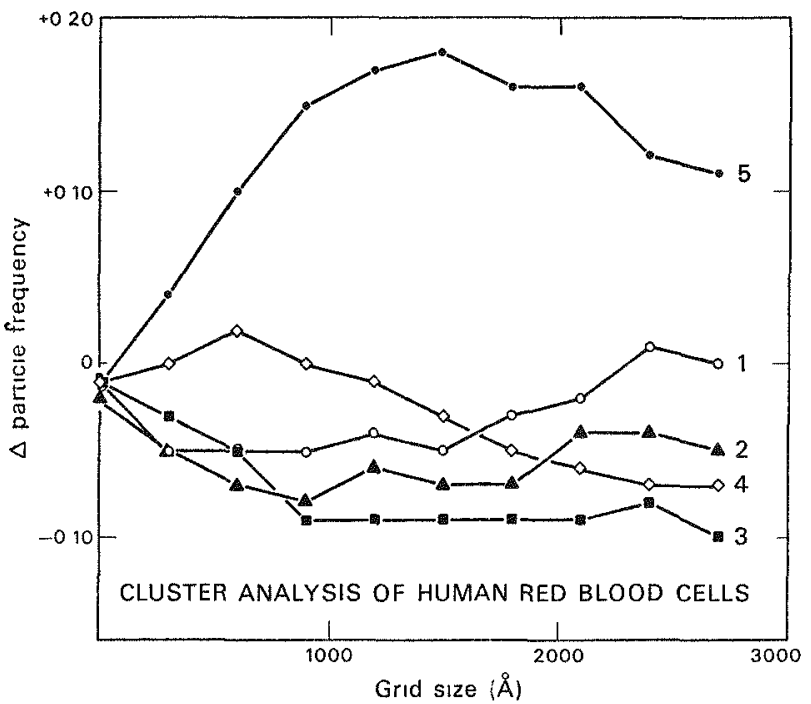

Fig. 8. Analysis of five electron micrographs of human red blood cells. Preparations \#1,2,3, 4 and 5 represent cells that were exposed to a progression of temperature treatments to induce particle aggregation. The significance of the curves is discussed in the text.

(XBL 761-346) showing that the finite particle size may lead to departures from the random point particle array. Rep1icas 4 and 5 are readily judged to be nonrandom. The maxima in the incremental frequency functions corresponding to these membranes provides a rough measure of the mean cluster size. Thus clusters in replica \#4 have an average diameter of about $600 \AA$ while those in replica $\# 5$ have larger diameters - about $1500 \AA$.

These results and others not cited here demonstrate that the pattern analysis procedure can be utilized for pictures that are amenable to subjective cluster identification. Work is in progress to extend pattern analys is to electron micrographs which are expected to exhibit more subtle aggregation patterns. Other studies are being pursued to examine the reproducibility of the derived parameters for different micrographs of the same membrane preparation.

\section{Footnote and References}

"Condensed from LBL-3203.

1. L. Packer and J. R. Smith, Proc. Nat. Acad. of Sci. 71:4763 (1974).

2. L. Hayflick, and P. S. Moorhead, Expt1. Ce11 Res. 25, 585 (1961).

3. D. W. Deamer, In vitro, 8, 428 (1973).

4. G. M. Maring, C. A. Sprague, and C. J. Epstein, Laboratory Investigation, 2n 23, 86 (1970).

5. A. L. Tappe1, Fed. Proc., 32, 1870 (1974).

6. D. llarman, J. Geront., 11, 298 (1956).

7. D. Harman, J. Geront., 16,247 (1961).

8. J, Epstein and D. Gershon, Mech. Age Dev. 1, 257 (1972).

9. L. Packer, J. S. Nolan, S. Katyare, and J. Smith, J. Bioenergetics, in press (1974).

10.S. Fleischer, H. Klourven, and G. Brierley, J. Biol. Chem., 236: 2936-2941, 1961.

11.L. Packer, Molecular Architecture of Energy Transducing Membranes Related to Function, in Biomembranes: Architecture, Biogenesis, Bioenergetics and Differentiation, L. Packer, ed., $147-166,1974$.

12.J. Torres-Pereira, R. Mehihorn, A. D. Keith, and L. Packer, Arch. Biochem. Biophys. 160: 90-99, 1974. 


\title{
Solar Energy Research and Development
}

\author{
INTRODUCTION
}

Solar energy includes a wide variety of meth ods by which the solar resource can be utillzed to provide for some of our energy requirements. It ranges from technologies that are we11 established and widely demonstrated to technologies that are now in the stage of basic research. This diversity is reflected in the National Solar Energy Program being conducted by ERDA, and in the program being conducted in the Energy G Environment Division of LBL. Within the Division there are ongoing projects in the areas of Solar Heating and Cooling of Burldings, Photovoltaic Conversion, Bioconversion, Solar Thermal Conversion, and Photochemical Conversion.

Most of the solar energy projects within the Division deal with technologies that are close to practical application. Projects related to technologies already in limited use include the design and control of solar heating and cooling systems for buildings, and the development of processes for the enzymatic conversion of cellulose (i.e., biomass) to fuels. In a project related to a near term but not yet demonstrated technology, measurements of the fraction of the solar radiation that can be utilized by concentrating solar collectors are being made to establish design criteria for facilities for the thermal conversion of solar energy to electricity. A project in the category of engineering research involves the design and development of engines that can convert the low temperature heat from solar collectors to mechanical motion in order to drive solar powered irrigation pumps, air conditioners, etc.

In the category of mission-oriented research is the project on polycrystalline solar cell materials. This project, which depends heavily upon resources made available by the Inorganic Materials Research Division, involves research on the deposition and electronic properties of polycrystalline silicon thin films, leading toward the development of low-cost solar cells.

Finally, still in the research stage is the project on the photochemical conversion of solar energy. This research involves developing an understanding of how the basic processes of photosynthesis might be adapted to the production of hydrogen from water.

\section{SOLAR CELL RESEARCH ${ }^{* \dagger}$}

Wigbert J. Siekhaus, Gabor A. Somorjai, Chin-An Chang, Jim Crump, Donald Tai-Chan Huo and Tom Kaminska

\section{INTRODUCTION}

Photovoltaic conversion of sunlight into electricity using solar cells is technically feasible. Efficient and stable solar cells are manufactured today from high-purity silicon single crystals sliced into thin $(0.3 \mathrm{~mm})$ rectangular wafers of approximately $10 \mathrm{~cm}^{2}$ area $\left(\sim 2 \times 10^{-2}\right.$ watt average output). However, the very high costs associated with the preparation and processing of single crystal silicon make this technology economically impractical. Our research is aimed at developing a polycrystalline silicon solar cell with suitable electrical properties in order to reduce the cost of photovoltaic energy conversion.

We are studying various methods of depositing layers of solar cell material with sufficient crystallinity over a large area $\left(\mathrm{m}^{2}\right)$. The catalysis of condensation and recrystallization of silicon doped with suitable impurities is being in-

stigated in order to find methods for the pro-

tion of thin silicon layers at low temperatures. ectron spectroscopy, low energy electron diffraction (LEED), and electron microscopy are being used to investigate the surface and grain boundary structure of thin films and the effect of this structure on electrical properties.
DEPOSITION AND CHARACTERIZATION OF SILICON FILMS

\section{Catalysis of Crystallization}

Recent work in this project and at other laboratories ${ }^{1}$ has been aimed at producing high crystallinity silicon and germanium films at rather low temperatures. Various metals were used in these studies to help recrystallize silicon and germanium. In our work we have used an ultra-thin aluminum coating (ca. $500 \AA$ ) before depositing silicon thin film onto fused quartz held at $600^{\circ} \mathrm{C}$. Silicon films thus produced showed much increased crystallinity compared to films directly deposited onto a quartz substrate. The process used was similar to the work of Filby and Nielson 2 with gold except that our work was done at a much lower temperature. Aluminum, which can be used only at these lower temperatures, is of particular interest because it gives p-type doping in silicon suitable for solar cells.

Before we applied the aluminum coating method to silicon film deposition we found that, at the vacuum of $1 \times 10^{-6}$ Torr that we used, aluminum films were unstable at $600^{\circ} \mathrm{C}$ either deposited directly onto the quartz substrate at this temperature or deposited at room temperature and heated to $600^{\circ} \mathrm{C}$ 
for $1 \mathrm{hr} .3$ Both oxidation and reaction with quart $z^{4}$ could be responsible for this observation. To overcome this difficulty a Si-Al-Si sandwich coating was used at a composition of about 100500-100 $\AA$. The inner silicon layer was to prevent aluminum reactions with quartz and the outer one to prevent aluminum oxidation. This $\mathrm{Si}-\mathrm{Al}-\mathrm{Si}$ coating was deposited onto quartz at room temperature. Upon heat treatment of this sandwich film at $660^{\circ} \mathrm{C}$, signs of melting were visually observable and the film turned pink in color. $X$-ray diffraction showed highly prefered (111)-oriented structures for both $\mathrm{Si}$ and $\mathrm{A} 1$. For comparison, no diffraction patterns were observed for either aluminum or silicon deposited at room temperature, silicon films deposited at $600^{\circ} \mathrm{C}$ showed only powder diffraction patterns, and aluminum film deposited at room. temperature and heat treated at $500^{\circ} \mathrm{C}$ for 2 hours showed highly preferred (111) orientation. These comparisons clearly indicated melting of silicon with aluminum in the Si-A1-Si sandwich film described above.

Next, silicon was deposited on top of the SiA1-Si coating at $600^{\circ} \mathrm{C}$. After deposition of about 2000-5000 \& thick silicon film, the substrate was heated to $660^{\circ} \mathrm{C}$ for 1 hour to allow better eutectic melting between silicon and aluminum. 5 The silicon film thus produced showed highly preferred (111) orientation and the relative intensity of (220)/(111) being 10-20\% compared with the value of $60 \%$ for the powder diffraction pattern. No diffraction pattern was observed for aluminum. Transmission electron microscopy showed a spot diffraction pattern, as shown in Fig. 1. From the diffraction pattern observed, a grain size of at least $5 \mu \mathrm{m}$ can be estimated.

In summary, we have demonstrated that an ultra thin layer of aluminum coated on fused quartz helped significantly to increase the crystallinity of silicon films deposited at fairly low temperatures. Work is now in progress to find the temperature dependence of the crystallinity increase. Other p-type and n-type metals will also be tested by this technique. We plan to combine this technique with that of chemical vapor deposition (CVD), using, for example silane, since CVD appears to be the most economical procedure for large scale production of silicon thin film solar cells.

Auger Analysis of the Interaction of Carbon with Silicon During Film Deposition

An important problem in thin film solar cells is the interaction between the substrate and the thin film. Carbon has been suggested as a suitable substrate for silicon solar cells; therefore, we have investigated the carbon-silicon film interaction.

Silicon films (2000-3000 \& thick) were vacuum deposited onto various allotropic forms of carbon (graphitic, amorphous, glass -1 ike) at $1150^{\circ} \mathrm{C}$ to test the possibility of using carbon as a substrate in thin film silicon solar cells. The distribution of silicon and carbon in the carbon-silicon interface was studied by using Auger spectrosopic depth profiling. The results showed that, for all substrates, silicon diffused deeply into
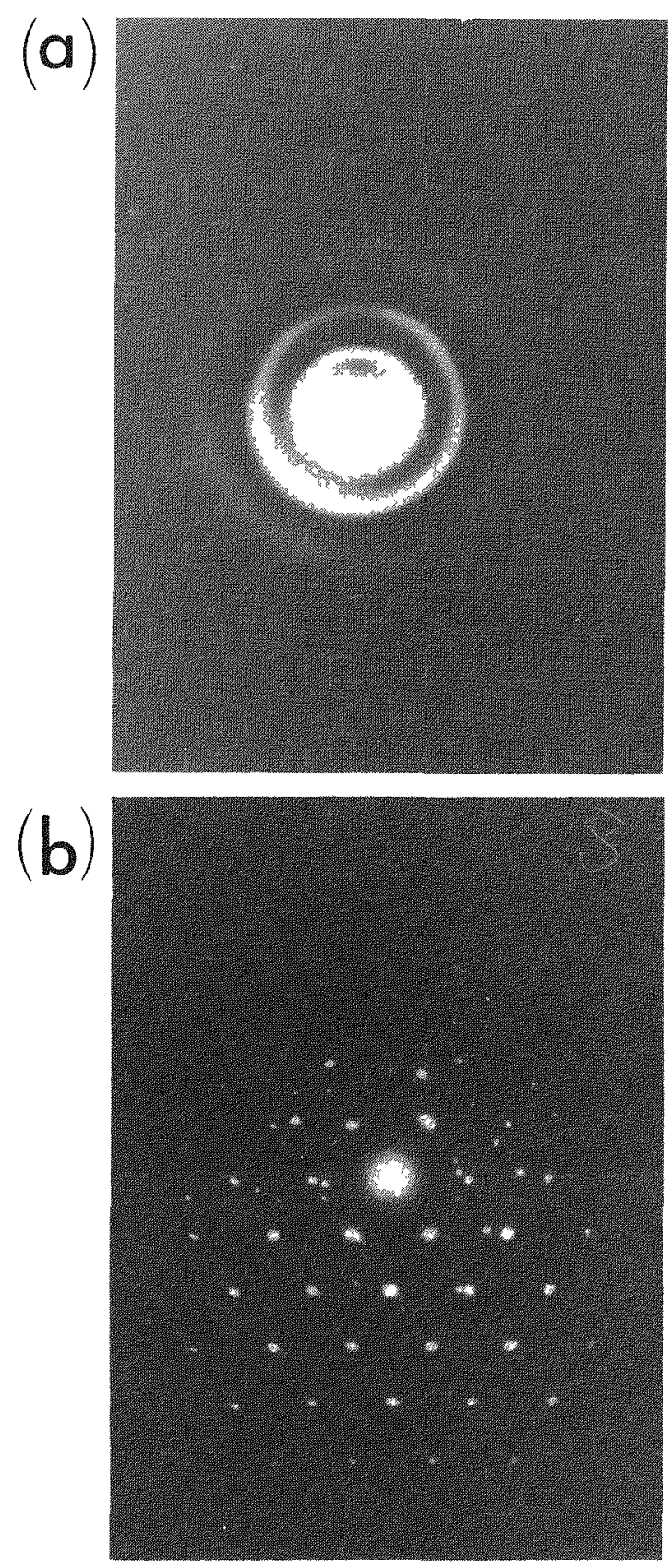

Fig. 1. Electron diffraction micrographs of (a) silicon film deposited on quartz at $600^{\circ} \mathrm{C}$; (b) silicon film deposited on quartz at $600^{\circ} \mathrm{C}$ with a prior coating of Si-A1-si layers. The crystallinity of the silicon film is enhanced by deposition on the SiAl-Si film. From the diffraction pattern observed in (b), a grain size of at least $5 \mu$ can be estimated. (XBB 749-6335)

carbon. In all cases, silicon carbide was formed.

Several methods of building diffusion barriers were tested. A diffusion mechanism was proposed according to which silicon atoms first form a carbide layer with the surface carbon atoms, followed by breaking up of the carbon lattice and diffusion of silicon through the carbide layers into the carbon substrates. This mechanism accurately predicts the dependence of the measured 
silicon and carbon contents on the structures of he carbon substrates used for depositions.

Effect of Sputtering on the Concentration Profile

Since argon sputtering is used as a principal tool in our work on the characterization of films, we are studing the effect of sputtering on the concentration profile. As a first step, the fate of the ions implanted during sputtering was studied theoretically as a function of the relative removal probability and the diffusion coefficient.

A theoretical solution has been given for the time dependent concentration of implanted ions lassumptions: constant diffusion coefficient in the range of penetration, $d(x)=\delta\left(x-x_{0}\right)$, $c(x=0, t)=0]$ together with general proof that the number of bombarding ions contained in a target at saturation is increased if the ions diffuse throughout the target, unless $c(0, t)=0$.

This solution has been used to determine the concentration profile and the total number of imbedded atoms at saturation as a function of a parameter $p$ (relative removal probability) and the diffusion constant $D_{0}(x)$. Measurements will show whether diffusion during sputtering is an important problem.

IDENTIFICATION OF IMPURITIES SEGREGATED ON GRATN BOUNDARIES AND THEIR EFFECT ON ELECTRONIC SURFACE STATES.

The review by Monch $^{6}$ and more recent work by Rowe and Ibach $^{7}$ show clearly that the large concentration of surface states that are traps and recombination centers for photoelectrons can be filled or decreased by orders of magnitude by exposure to various adsorbates. This phenomenon will be of importance for the development of polycrystalline solar cells since proper treatment of the polycrystalline interfaces will be required to enhance ce11 performance.

Knowledge of the influence of the physical structure of the surface on surface states is required for an understanding of this phenomenon. To study one aspect of this problem on a well controlled system, a Leed-Auger study of the Si(111) surface has been undertaken with the aim of gathering information on the reconstructed surface that gives a $(7 \times 7)$ LEED pattern. This surface reconstruction has been seen by many observers using samples which cover a wide range of doping levels. We have found that at background pressures between $5 \cdot 10^{-10}$ and $5 \cdot 10^{-9}$ the reconstruction proceeds rapidly above $700^{\circ} \mathrm{C}$, producing the well-defined $(7 \times 7)$ diffraction pattern.

Analysis of the (00) LEED beam intensity versus voltage curves for both unreconstructed and reconstructed surfaces has shown distinctive and ntrasting features as illustrated in Fig. 2. So $r$ the comparison of the $(1 \times 1)$ and $(7 \times 7)$ inCensity versus voltage curves has been restricted to the 50-150 volt region, because at higher energies the pattern for the $(1 \times 1)$ surface is poorly resolved. For the $(7 \times 7)$ surface, the study is easily extended to 300 volts.

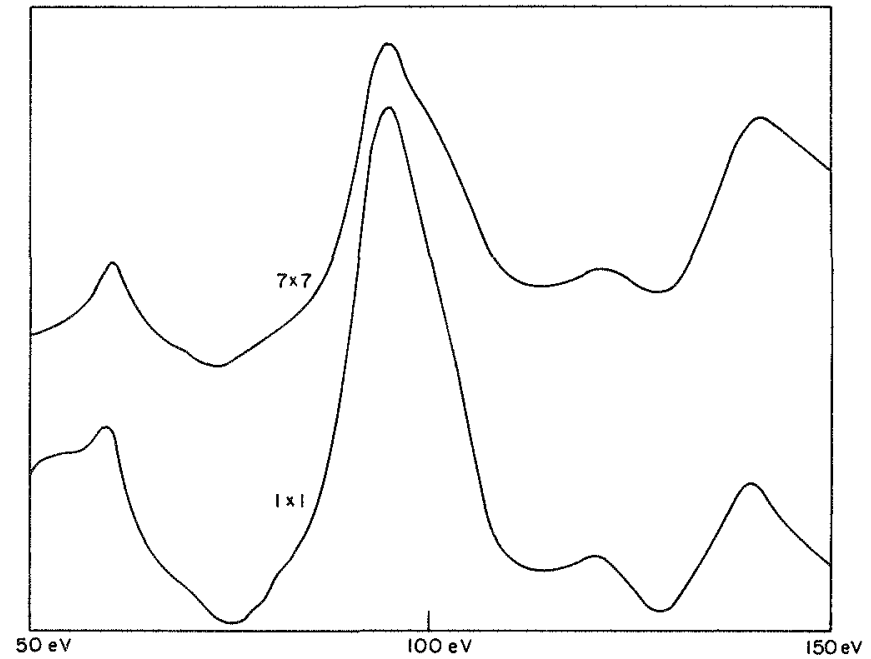

Fig. 2. The (00) beam intensity vs voltage curves for the $(1 \times 1)$ and $(7 \times 7)$ surfaces in the range $50-150 \mathrm{eV}$, taken at an incident angle of $4^{\circ}$. The azimuthal angle was $30^{\circ}$. Peaks appear at 47-57, 96,117 and $137 \mathrm{eV}$. In this range, reconstruction to give the $(7 \times 7)$ surface alters the relative magnitudes of the peaks. The major difference between the two curves is the increased intensity of the peak at $137 \mathrm{eV}$ relative to the intensity of the peaks at 47-57 eV with reconstruction. The voltages are the "external" values, not corrected for contact potential differences or for inner potential.

(XBL 7412-7675)

A comparison of the temperature dependence of the various intensity maxima of diffraction beams from the $(1 \times 1)$ and the $(7 \times 7)$ structure has been completed. The results show that surface reconstruction causes a measurable change in the surface Debye-Waller factor, and hence, by the harmonic oscillator mode1, a change in the mean square displacement of top layer atoms in the direction normal to the surface. This measured change in structure and bonding corresponds to and confirms observed changes in surface states reported in the iterature. 7

We are now examining the effect of impurities on surface states in polycrystalline silicon. Polycrystalline samples of electric and metallurgical grade silicon (either before or after they have been diffused to form pn junctions) are being broken along grain boundaries under ultrahigh vacuum conditions. Impurities on the grain boundaries are being identified (qualitatively and quantitatively) by use of Auger electron spectroscopy. Single-crystal silicon samples with low-Millerindex surfaces will be exposed under ultrahigh vacuum conditions to various impurities. The effect of impurities on surface states will be studied by high resolution electron loss spectroscopy and photoelectron spectroscopy.

\section{FOOTNOTES AND REFERENCES}

* Condensed in part from: Chin-An Change, wigbert $J$. Siekhaus, Thomas Kaminska and Donald Tai-Chan Huo, "Enhanced Crystallinity of Silicon Film Deposited at 
Low Temperature". LBL-3165 (Sept. 1974), Wigbert J. Siekhaus, "The Effect of Diffusion and Relative Removal Probability on the Total Number and on The Concentration Profile of Ion's Imbedded into a Target During Sputtering", LBL-3205, (Sept. 1974), and James G. Crump, "Mean Square Displacement of Surface Atoms on the Silicon (111) Crystal Face", LBL-3575, (In Preparation).

Work supported by the National Science FoundationRANN Division.

1. J. O. McCaldin and H. Sankur, App1. Phys. Lett. 20, 171 (1972); H. Sankur, J. 0. McCaldin and J. Devaney, App1. Phys. Lett. 22, 64 (1973); G. Ottaviani, D. Sigurd, V. Marre11o, J. O.
McCaldin and J. W. Mayer, Science 180, 948 (1973).

2. J. D. Filby and S. Nielsen, J. Electrochem. Soc. 112,957 (1965).

3. Chin-An Chang, unpublished results.

4. K. Prabiputaloong and M. R. Piggott, J. Electrochem. Soc. 121, 430 (1974).

5. R. Hultgren, P. D. Desai, D. T. Hawkins, M. Gieser and K. K. Kelley, Selected Values of the Thermodynamic Properties of Binary Alloys (American Society of Metals, 1973).

6. Winfried Monch, "On the Physics of Clean Silicon Surfaces", XIII Advances in Solid State Physics, Pergamon Press, March 1973.

7. J. E. Rowe and H. Ibach, Phys. Rev. Letters, 9 July 1973, Vol. 31, No. 2 .

CHLOROPLAST MEMBRANE STRUCTURE AND PHOTOSYNTHETIC ELECTRON TRANSPORT STABILITY*
G. Papageorgiou,
G. D. Case, S. Hansen, and L. Packer

Hydrogen production from water and sunlight by the photosynthetic machinery of plants or algae could become a nondepletable and nonpolluting energy source suited for technological societies. However, the photosynthetic hydrogen production mechanisms demonstrated until now are not yet of proven suitability for light energy conversion systems. One of the main problems encountered is the instability of photosynthetic reactions, particularily the water splitting reaction. In the course of a project directed toward the development of efficient and stable solar energy conversion systems based on photosynthesis, we have addressed this problem. In particular, we have conducted investigations related to the stability of chloroplast membranes.

There is a growing awareness of the importance of chloroplast membrane structure to photosynthetic electron transport reactions and to the stability of photosynthetic functions. Electrochemical gradients across thylakoid membranes are implicated 1,2 in photosynthetic energy conservation, which can be driven by either cyclic or non-cyclic electron transfer. However, the formation of reducing equivalents from $\mathrm{H}_{2} \mathrm{O}$ requires non-cyclic electron flow through two 1 ight reactions, which are separated spatially from one another in the membrane. Spatial separation may be advantageous because photolytically generated $\mathrm{O}_{2}$ may oxidize and inactivate many low-potential iron sulfur centers such as ferredoxin and hydrogenase. 3 other deleterious effects of oxygen on membrane 1 ipids have also been reported. 4 Separation of the photosystems may confer increased stability to system I relative to system II, if $\mathrm{O}_{2}$ effects represent an important mechanism of chloroplast deterioration. The chloroplast membranes may also provide a two-dimensional "solid state" matrix for the electron transfer reaction. Changes in the spatial arrangement of membrane components may therefore generate changes in photosynthetic functions.

We are examining the effects of agents that alter the structure and integrity of chloroplast membranes on the stability of various photosyn- thetic functions. It is already known that antioxidants, fatty acid scavengers, 5 and crosslinking agents such as glutaraldehyde and imidoiesters 0,7 can prolong the useful life of chloroplasts in vitro. The results presented here extend these observations in order to ascertain the interrelationship of membrane structure and membrane function more directly.

Chloroplasts were prepared from fresh market spinach according to established procedures. Crosslinking with dimethyl suberimidate (DMS) was carried out either with 0.01 mmole DMS/mg chlorophyll for $15 \mathrm{~min}$ at $25^{\circ} \mathrm{C}$ (Table 1), or with higher levels of fixative at $4^{\circ} \mathrm{C}$ overnight. Light-induced energization of chloroplasts was followed by measurements of atebrine fluorescence quenching, and light induced osmotic responses by light-scattering at $546 \mathrm{~nm}$. Electron transport through photosystem II was monitored spectrophotometrically as $\mathrm{K}_{3} \mathrm{Fe}(\mathrm{CN})_{6}$ or DCPIP reduction, or polarographically as $\mathrm{O}_{2}$ evolution. Electron transport through photosystem $\mathrm{I}$ was determined polarographically as $\mathrm{O}_{2}$ uptake in the presence of $4 \mathrm{mM}$ ascorbate, $26 \mathrm{~mm}$ DCPIP, $2 \mathrm{mM} \mathrm{NaN}_{3}, 20 \mu \mathrm{M} \mathrm{DCMU}$, and $100 \mu \mathrm{M}$ methy1 viologen.

Table 1 presents a preliminary experiment on the effects of DMS on photosystem II electron transport with either a physiological electron donor $\left(\mathrm{H}_{2} \mathrm{O}\right)$ or an artificial one $(1,5-$ dipheny1carbazide; DPC). The data clearly demonstrate the instability of the water splitting reaction, and the apparent ability of DMS to prolong it. Photoreaction II alone is a good deal more stable than the water splitting reaction (Table 1 ), as is the photosystem I (P700) light reaction (data not shown). Experiments using $\mathrm{K}_{3} \mathrm{Fe}(\mathrm{CN})_{6}$ as electron acceptor gave results similar to those shown in Table 1 .

Fixation at room temperature at levels in ex cess of 0.01 mole DMS/mg chlorophyll obliterates both the water-splitting reaction and the osmotic response of chloroplasts. On the other hand, chloroplasts reacted overnight at $0-4^{\circ} \mathrm{C}$ with DMS levels as high as 0.2 moles/mg chlorophy1l retain their 
Table 1. DCPIP photoreduction from $\mathrm{H}_{2} \mathrm{O}$ or diphenylrbazide

\begin{tabular}{|c|c|c|c|c|}
\hline \multirow{2}{*}{$\begin{array}{l}\text { Storage time } \\
\text { at } 25^{\circ} \mathrm{C} \text { (min) }\end{array}$} & \multicolumn{2}{|c|}{$\begin{array}{l}\text { DCPIP reduced } \\
\text { from } \mathrm{H}_{2} \mathrm{O}\end{array}$} & \multicolumn{2}{|c|}{$\begin{array}{l}\text { umoles ( } \mathrm{min} \mathrm{mg} \text { che } \\
\text { from DPC }\end{array}$} \\
\hline & $+\overline{\mathrm{MS}}$ & -DMS & $+\mathrm{DMS}^{2}$ & -DMS \\
\hline $120^{0}$ & $\begin{array}{l}6.8 \\
1.8\end{array}$ & $\begin{array}{l}4.8 \\
0.0\end{array}$ & $\begin{array}{l}4.8 \\
4.8\end{array}$ & $\begin{array}{l}3.0 \\
3.6\end{array}$ \\
\hline
\end{tabular}

ability for photoinduced electron transport across photosystem II and photosystem I, although they have lost their osmotic response completely. Indeed, such preparations are characterized by electron transport rates higher than those of the unfixed controls, suggesting the possibility that either DMS, or one of its hydrolysis products, may be an uncoupler.

Chloroplasts fixed with DMS at low temperature continue to transport $\mathrm{H}^{+}$ions across the thylakoid membrane, as indicated by their ability to quench the fluorescence of atebrine when illuminated with strong actinic light. Concentration curves show that electron transport, atebrin. fluorescence quenching, and loss of osmotic response are maximized at a level of 0.05 mmoles DMS/mg chlorophy11. (nolar ratio, DMS/chlorophy11 $\simeq 1 / 20$ ).

Stable as photosystem I electron transport is, its requirement for an intact membrane system is apparent from Fig. 1. This experiment demonstrates

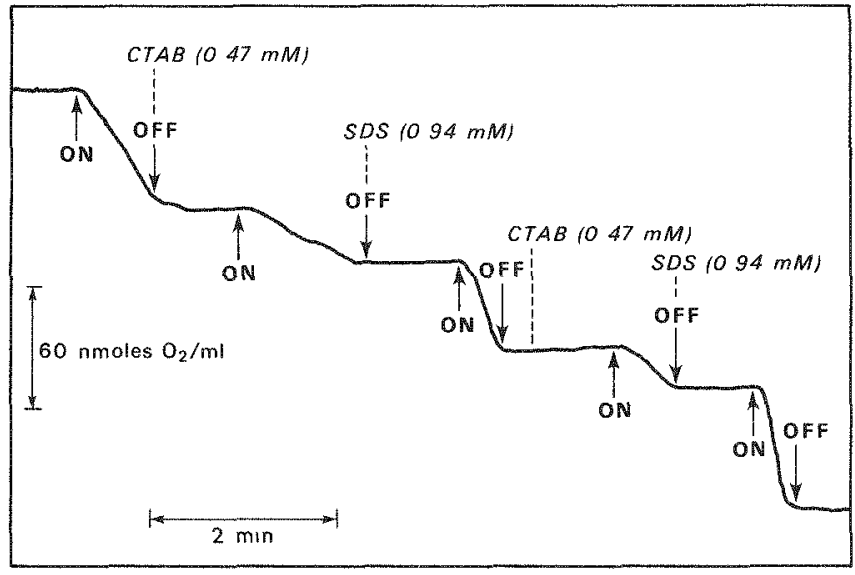

Fig. 1. Polarogram showing the effects of the cationic detergent $C T A B$ and the anionic detergent SDS on photosystem I electron transport of DMSimmobilized chloroplasts. The reaction mixture contained $16 \mu \mathrm{g} \mathrm{Ch1} / \mathrm{ml}$; sodium ascorbate, $4 \mathrm{mM}$; DCPIP, $26 \mu \mathrm{M}$; $\mathrm{NaN}_{3}, 2 \mathrm{mM}$; DCMU, $20 \mu \mathrm{M}$; plus the indicated concentrations of the detergents. The ordinate indicates the amount of light driven $\mathrm{O}_{2}$ take from ascorbate. On and off arrows demarcate ht and dark periods. Dashed lines indicate times detergent addition. After each addition the reaction mixture was incubated for 1 minute in the dark. The rate of $\mathrm{O}_{2}$ uptake decreases upon addition of detergent; effect is reversed by addition of a counter-ion detergent. the reversibility of certain structural perturbations in biological membranes. On the addition of cationic detergent cetyl trimethyl ammonium bromide (CTAB), photosystem I activity of fixed chloroplasts is diminished by about $60 \%$. On subsequent addition of the anionic detergent sodium dodecyl sulfate (SDS) the activity is restored to even higher levels. One can repeat this cycle several times, but eventually a point is reached beyond which the detergent counter-ion reversal is no longer possible. Detergent effects on photosystem II reactions are currently being investigated. These effects emphasize the importance of the lipid part of the membrane in the overall expression of such complex functions as the photosynthetic electron transport. Provided that the detergent solubilization of membrane lipids is not extensive, the original function can be restored by neutralizing the perturbant with a detergent counter-ion. Similar observations from this laboratory ${ }^{8}$ with other biological membranes suggest that the cation-anion detergent complex remains in the reconstituted membrane.

At lower concentrations (ca. 20-30 $\mu \mathrm{M}$ ) CTAB quenches chlorophyl1 a fluorescence. This effect, which is not reversed by SDS, indicates that the cationic detergent can penetrate to the pigment phase, where it quenches by functioning as an excitation sink. This property is common to many electron deficient agents, such as oxygen, quinones, nitroaromatic compounds, and quaternary ammonium cations.

In summary, we have demonstrated the importance of an intact membrane structure in photosynthetic electron transport. We have also shown that immobilization of chloroplasts with crosslinking agents increases the stability of photosynthetic functions. We are presently investigating the molecular mechanisms of chloroplast photosynthetic instability, and the mechanism of action of immobilization.

\section{FOOTNOTES AND REFERENCES}

${ }^{*}$ Condensed, in part, from LBL-3213.

${ }^{\dagger}$ On 1eave from the Nuclear Research Center

"Democritus", Athens, Greece.

1. P. Mitche11, Chemiosmotic Coupling in Oxidative and Photosynthetic Phosphorylation, Glym Research Ltd., Bodwin, Cornwall (1966).

2. D. W. Deamer, A. R.Crofts, and L. Packers, Biochim. Biophys. Acta 131, 81 (1967).

3. J. R. Beneman, J. A. Berenson, N. O. Kaplan, and M. D. Kamen, Proc. Nat1. Acad. Sci. U.S.70, 2317 (1973).

4. R. L. Heath and L. Packer, Arch. Biochem. Biophys. 125,$189 ; 850$ (1968).

5. T. Takaoki, J. Torres-Pereira, and L. Packer, Biochim. Biophys. Acta 352, 260 (1974).

6. J. West and L. Packer, Bioenergetics 1, 405 (1970).

7. L. Packer, J. Torres-Pereira, P. Chung, and S. Hansen, Proceed. 3rd Intern. Congress of Photosynthesis, Rehovot, Israel (1974).

8. R. Mehlhorn and L. Packer, this report (1975). 


\section{SOLAR HEATING AND COOLING OF BUILDINGS*}

M. Wahlig, E. Binnal1, R. Graven, F. Selph, R. Shaw, M. Simmons, J. Tanabe, and R. Wolgast

The primary objective of this project is the development of an inexpensive electronic control system that will operate a solar combined heating and cooling system in an optimized way. This controller would replace the simple thermostat control of conventional heating and cooling (H/C) systems. A second objective is the development of an ammonia-water absorption air conditioner that will provide the cooling for the combined $\mathrm{H} / \mathrm{C}$ system. And a third objective is the establishment of a network of solar radiation measurement stations that will accumulate data on the incident solar flux (insolation) at various locations in the central and northerm California area serviced by the Pacific Gas and Electric Company (PGEE). These measurements, being carried out in collaboration with $\mathrm{PGEE}$, will provide data necessary for calculating the economic feasibility of solar $\mathrm{H} / \mathrm{C}$ systems in this area.

Different solar $\mathrm{H} / \mathrm{C}$ configurations and different operating strategies will be optimal for different climatic regions. To ensure widespread applicability of the control system, it is being designed to operate essentially all practical $\mathrm{H} / \mathrm{C}$ system configurations simply by interchanging replaceable modular units in the circuitry.

Such an experimental $\mathrm{H} / \mathrm{C}$ system, the main purpose of which is to allow testing and exercise of the controller, has been designed to be operated in 211 practical configurations. The main system, shown schematically in Fig. 1, has 26 different practical operating configurations. Any of these can be selected electronically (by the controller) by choosing the states of the solenoid valves (V1, V2, etc.), the pumps (P1, P2), and the auxiliary heater (A1). This system is being installed at LBL in Building 25A, with its solar collectors on the roof, the storage tank on the main floor, and most of the plumbing and controls on the balcony. This building was chosen primarily because its roof offers the Laboratory's best solar exposure for the collectors, and secondarily because the large interior volume allows free experimentation with the solar system, without appreciably disturbing the working environment of the building.

The collectors consist of 18 separate pane1s comprisjng a total of approximately $320 \mathrm{ft}^{2}$ (purchased from PPG Industries, Inc.). They are mounted in such a way that their tilt may easily be set at any angle between $30^{\circ}$ and $60^{\circ}$. The storage tank has a 3000 gallon capacity, and is of 16gauge galvanized steel. The working fluid is water with corrosion inhibitors. Serious consideration was given to using anti-freeze (ethylene glycol solution) as the collector fluid, with a heat exchanger between the collectors and the storage tank. This idea was rejected because the controller operation would be identical with or without the heat exchanger, making the extra

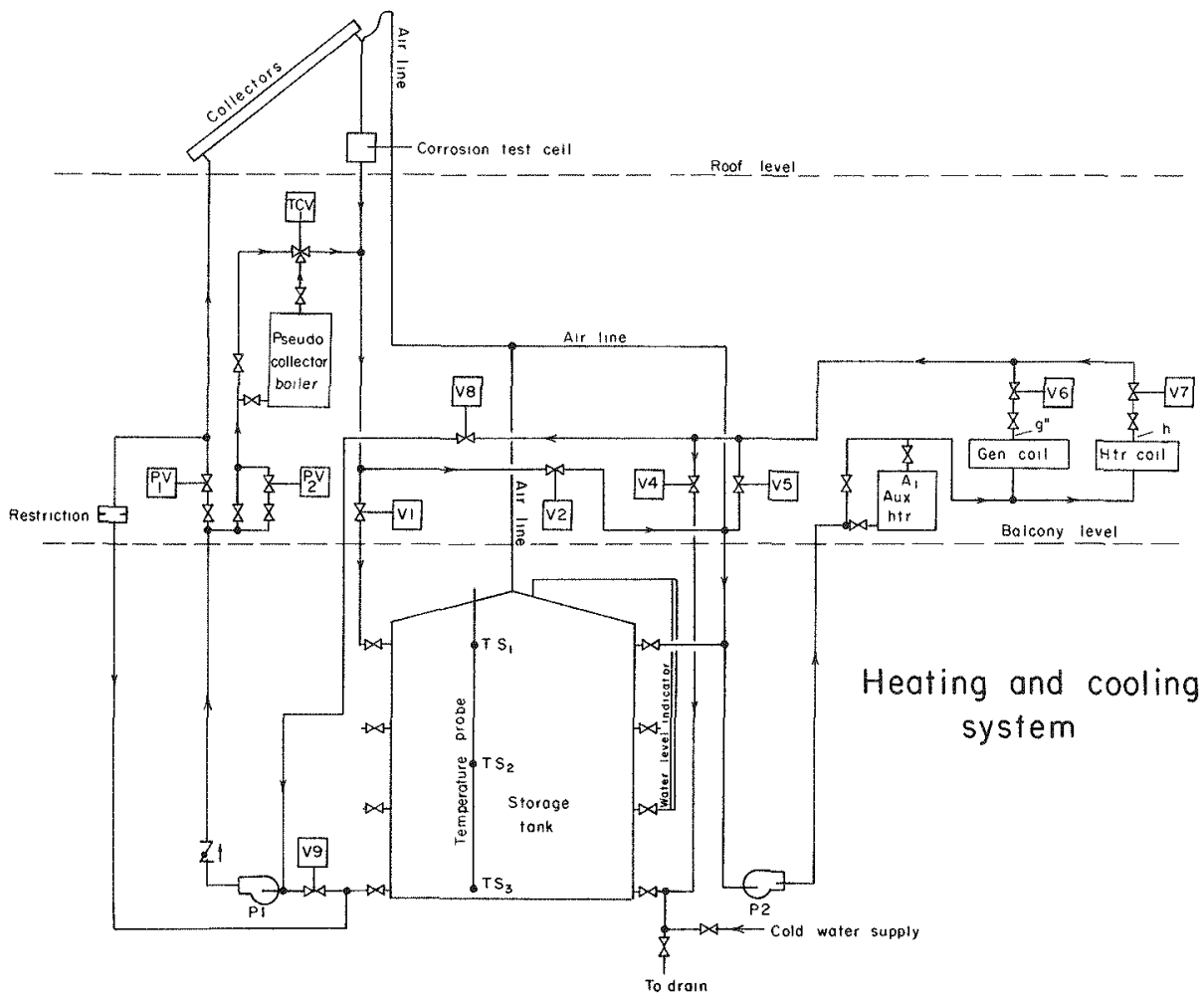

Fig. 1. Main solar heating and cooling system. The system has 26 different operating configurations. Any of these can be selected by choosing the states of the solenoid valves (V1,V2, etc.), the pumps (P1,P2), and the auxiliary heater (A1).

(XBL. 751-2178) 
expense unwarranted. However, the choice of water is the collector fluid requires the development of a fool-proof drain system to prevent freezing damage. The restriction shown on the left in Fig. 1 was designed for this purpose, and will be thoroughly tested. Nomal water flow rates through pumps P1 and P2 will be about 10 gallons per minute.

Since the primary purpose of the experimental system is to test the controller, we decided not to use a complete set (typically $800 \mathrm{ft}^{2}$ ) of collectors. Instead, only about $1 / 3$ of the flow passes through the collectors, and about $2 / 3$ passes through the "pseudo-collectors." A separate, selfcontained control system manages the flow through the boiler in the pseudo-collector 1ine, producing an output fluid temperature that can duplicate that of the collectors, or it can boost the collector fluid output temperature if desired. This pseudo-collector feature not only reduces by a factor of 3 the roof collector area that must be maintained, but more importantly allows experimentation with the rest of the system and the controller even when there is no sunshine. It should be emphasized that this pseudo-collector circuit is only an experimental tool; it would not exist in an actual residential solar $\mathrm{H} / \mathrm{C}$ system, and it does not interact with the controller.

The loads on the main system are subsystems for heating and cooling the building air and a subsystem for heating the domestic hot water. The domestic hot water system shown in Fig. 2 can be operated by using only one tank $\left(\mathrm{HW}_{1}\right)$ or both tanks. In the latter case, gas heat would be used, when necessary, to boost the temperature of only the second tank, $\mathrm{HW}_{2}$; the first tank would then be used only as a solar preheater. Control algorithms have been formulated to rum both the onetank and two-tank configurations. The relative economics of the two methods will be determined experimenta11y.

In order to assess the system's air heating and cooling performance accurately, controlled load (pseudo-load) systems have been devised. These are shown in Fig. 3. The first of these simulates the building air flow system and allows, in a controlled and measurable fashion, the transfer of heat from the heating coil or to the cooling coil. The second pseudo-load system simulates the outside air, and permits controlled air flows across the absorber and condenser coils of the air conditioner. This allows determination of the air conditioner performance under standard test conditions of indoor and outdoor air temperatures.

The air conditioner is an Arkla gas-fired ammonia-water chiller, modified for operation with solar-heated water. We decided that such modification of an existing commercial unit was more practical, given constraints of the present contract, than the fabrication from scratch of a complete solar-driven air conditioner. However, much of the design for a complete unit has been done and is contained in LBL Engineering Notes.1

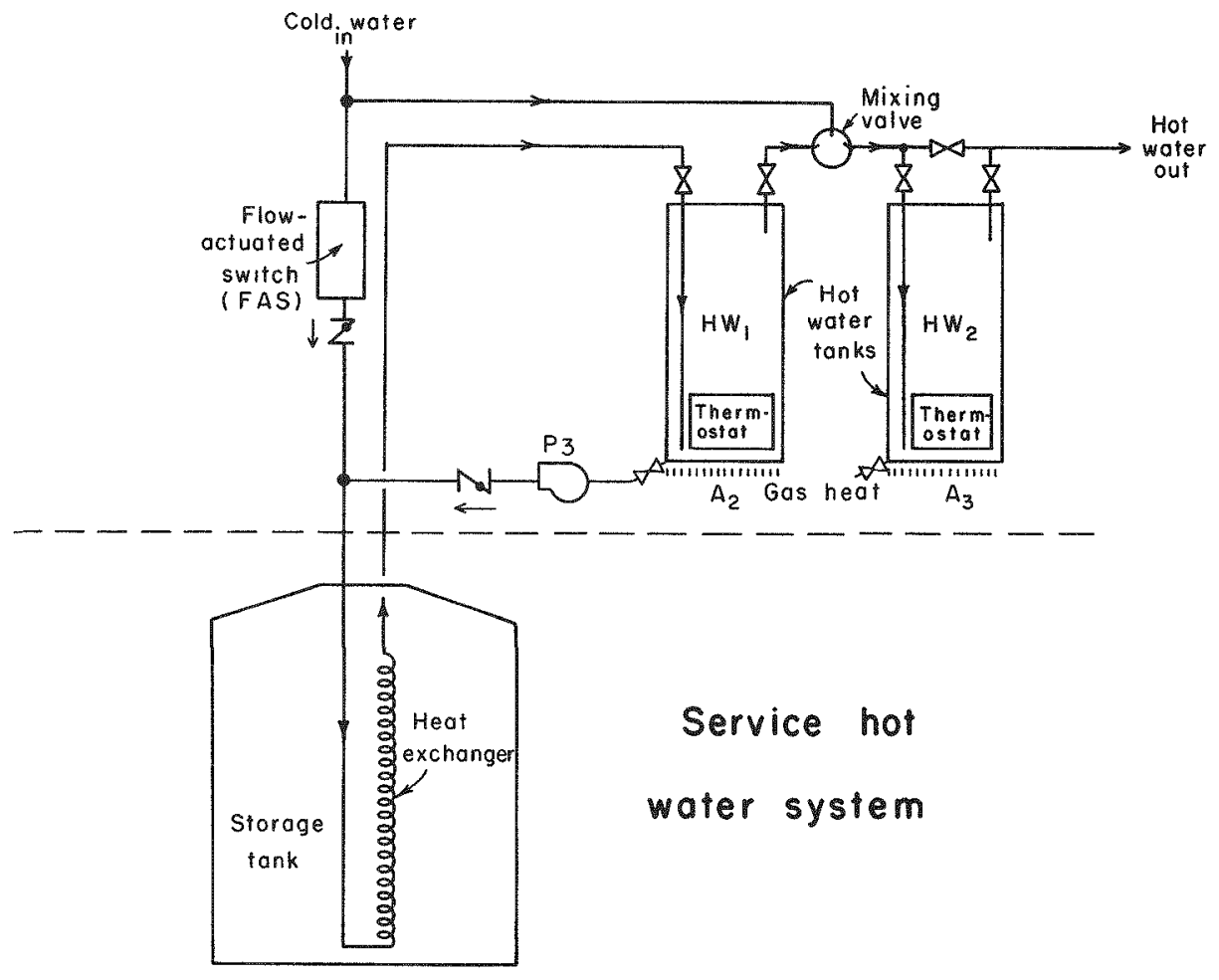

Fig. 2. Solar heated domestic hot water system. The system can be operated using only one tank $\left(\mathrm{HW}_{1}\right)$ or using both tanks. When both tanks are used, auxiliary heat is applied to $H W_{2}$ when necessary; $H W_{1}$ is used as a solar preheater only.

(XBL, 751-2179) 


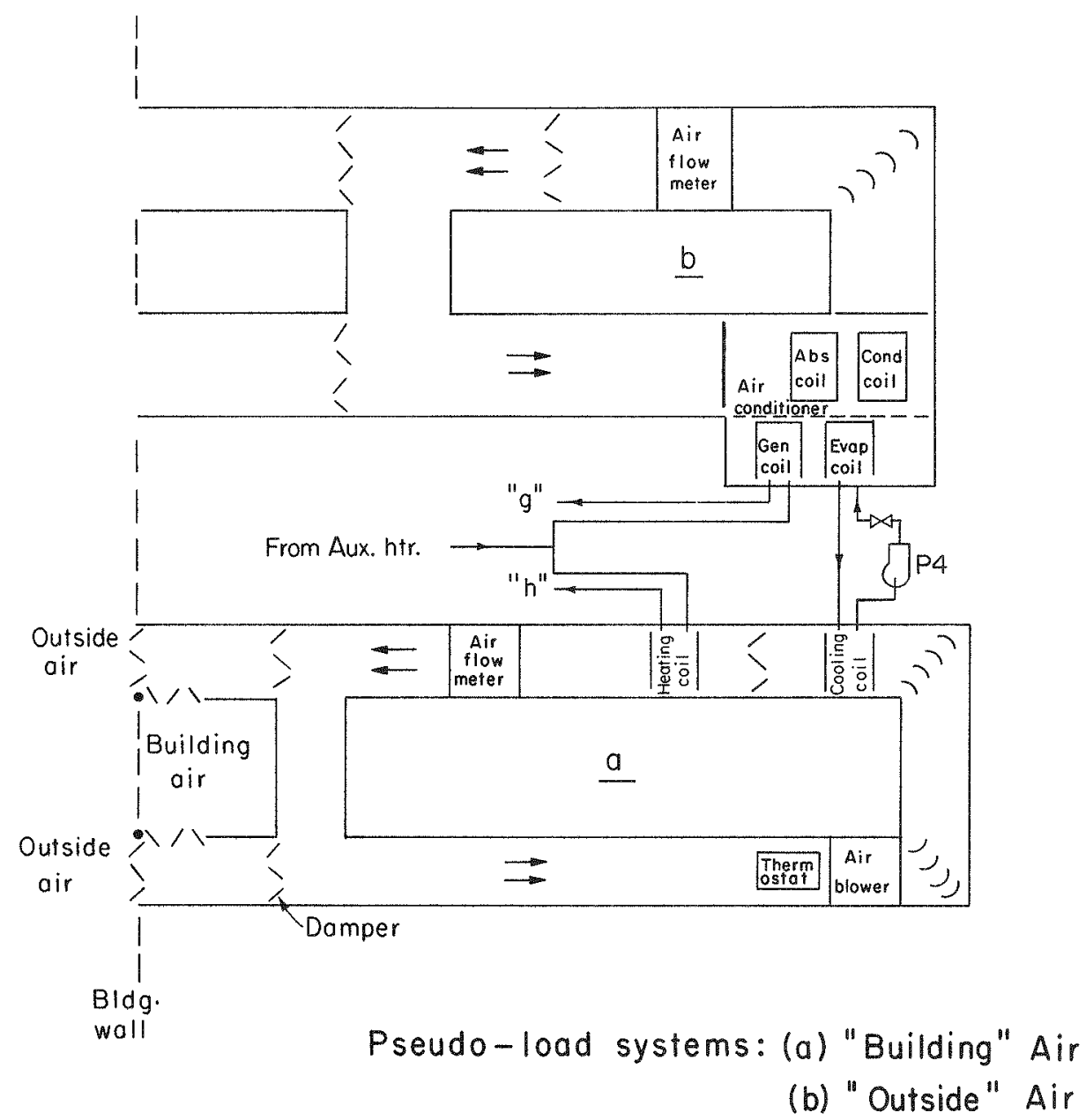

Fig. 3. Controlled load (pseudo-load) solar air heating and cooling systems. System (a) simulates the building air flow system and allows the transfer of heat from the heating coil or to the cooling coil. System (b) simulates the outside air and permits controlled air flows across the absorber and condenser coils of the air conditioner. (XBL 751-2179)

At present, all major pieces of equipment have been ordered and most are already on hand. The collector support structure has been designed and collectors are being installed on the roof. The storage tank has been modified for extra outlets, and is being installed and foam-insulated on the floor of Building 25A. The system is being completely instrumented with temperature, pressure, and flow indicators to monitor system performance.

The generalized H/C system of Figs. 1,2, and 3 contains we11-defined control units (pumps, valves, auxiliary heaters) that will serve as the output devices of the electronic controller. Solid-state temperature sensors will be installed at critical (i.e., decision-making) locations in the system and will serve as inputs to the controller; our current design calls for nine of these. Algorithms are being developed that will define the logical operation of the controller. The algorithms will be provided to the controller circuit in the form of programmable read-onlymemory chips, facilitating the use of different algorithms to run the experimental $\mathrm{H} / \mathrm{C}$ system in different configurations. A single residential system in a given climate region would operate only according to a single algorithm and would only include a small subset of the temperature sensors and control valves shown in our generalized experimental system. The input and output units for the controller have been fully specified, and the electronic hardware for the processor is now being designed.

Plans for the solar insolation measurement stations include the use of home-made and commercial pyranometers, LBL-designed and fabricated interface circuits, and $\mathrm{PGEE}$ recorders. For the initial set of six stations, we decided to use Eppley Black and White Pyranometers, and these have been procured. PGGE is providing some of their billing ratemeters for the data recording. Most or all of these will use slow-speed, twotrack magnetic tapes, with a time mark on one track and the solar insolation data on the other. The interface circuit feeds the pyranometer output level into a voltage-to-frequency converter, the output of which is written onto the magnetic tape. This interface has been designed, and a prototype unit has been fabricated and debugged, and is now undergoing temperature-stability tests. 
After the Six solar insolation stations have en set up, the magnetic tapes will be collected eriodically and shipped to PG\&E in San Francisco for initial processing of the data onto standard digital magnetic tapes. Final data analysis will then be done at LBL. The tentative locations for the first six measurement statıons are LBL, Oakland, Fresno, Bakersfield, Redding and Sacramento.

\section{FOOTNOTE AND REFERENCE}

*Work supported by the National Science FoundationRANN Division.

1. LBL Engıneering Notes, Code EE0904, Serıa1 M4708, M4744, M4754, and M4755.

\section{NITINOL ENGINL PROJECT}

R. Banks, J. Gunn, P. Hernandez, D. Johnson, H. Mohamed R. Nickerson, D. Norgren, J. Washburn, O. Weres

\section{INTRODUCTION}

The purpose of this project is the development of the technology required for constructing practical heat engines capable of operating on small temperature differences at near ambient temperature. Our efforts have been focused on solid-state engines that depend for their operation on the thermally related shape memory properties of certain nickel-titanium alloys. The first successful application of these materials to a continuously operating heat engine was demonstrated at the Lawrence Berkeley Laboratory in August 1973.1 Since that time parallel programs in materials study, thermodynamic analysis, prototype concepts and design of $\mathrm{N}_{1}-\mathrm{T}_{1}$ heat engines have been initiated at LBL.

\section{NI IINOL}

The sexies of near equiatomic NickelTitanium intermeta111c compounds known as 55 Nitino ${ }^{2}$ have been known for several years to exhib1t energetic mechanical shape-memory responses related to a solid-state phase transformation triggered by elther thermal or mechanical (stress) influences. The two phases of particular interest, designated as NI-TI II and N1-TI III-or, w1 th reference to analogous formations in steels, as the austenitic and martensitic phases-are distinguised by differences in crystalline structure that dre interconverted via a diffusionless shear mechanism.

The cumulative effect of these shear-displacements produces macroscopic changes in the mechanical (as wel1 as many other) properties of the material such that in the lower temperature state it may be readily deformed, while at higher temperatures it exhibits a nomal degree of elastic1ty. In the higher-temperature phase a specimen may be imprinted with a physical shape "memory" by hedt treatment, and w1 11 spontaneously return to that shape on heating above the N1-Ti II threshold. When the material is deformed at the low temperature, heated and allowed to return to 1 ts imprinted shape, net mechanical work is produced. 3 This phenomenon enables 55-NItinol to act as a solid state thermomechanical transducer. After an engine element has

en cycled for several hundred (or thousand, deiding on the engine) cycles, it begins to return a definite cold temperature shape without the application of stress. In other words, in addition to a hot shape memory, it also develops a cold shape memory. This behavior, which we call "double training," is very desirable in engine elements.

\section{MATERIALS STUDY}

Selected-area electron diffraction and transmission microscopy were used to study the NI-TI II/NI-TI III transformation. All investigators have agreed that the high-temperature phase has a simple CsCl structure, but regarding the Jowtemperdture phase (martensite) there has been a great deal of uncertanty. The selected-area diffraction patterns obtained from martensitic domains suggest that the unit cel1 has orthorhombic symmetry and thus there are six equivalent Bain distortions. This implies that the martensite will form with many variants which are crystallograph1cally equivalent and randomly oriented. Figure 1 is an example of the generdl appearance of the microstructure, it can be seen that the mortensite domains are randomly oriented.

The bands within each martensite plate wcre proved to be twan related (1.e., twiming rather than slip furnishes the lattice invariant deformation in a partially transfomed material), it can be seen in Fig. 1 that some of the twin related do-

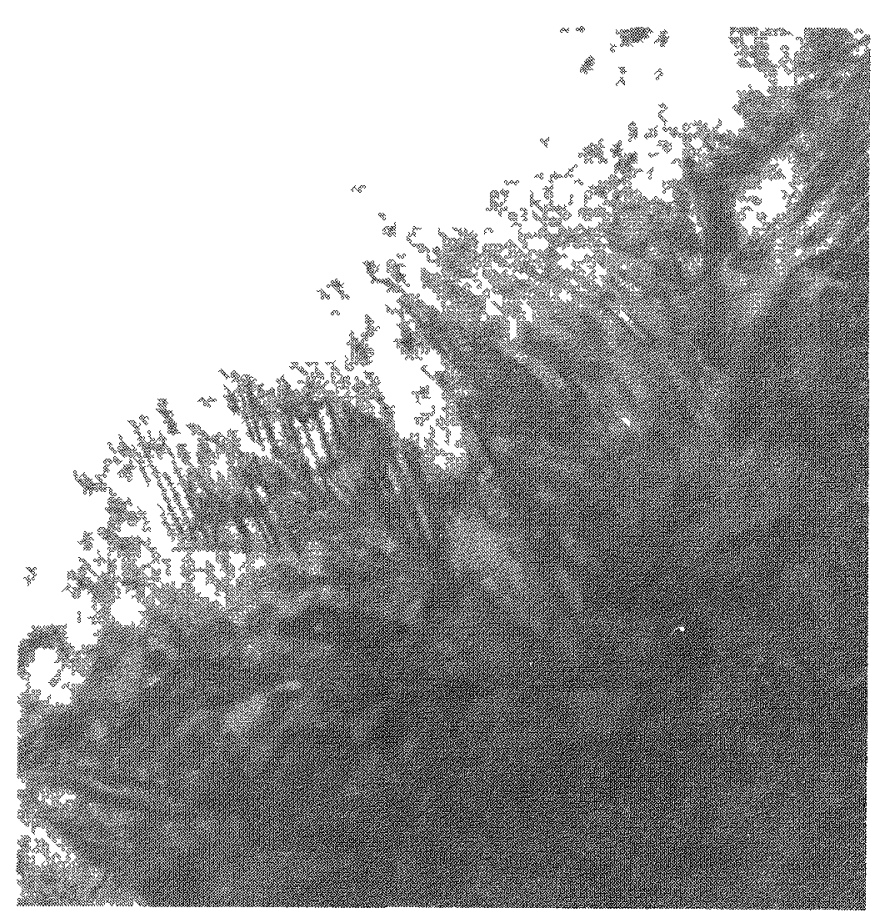

Fig. 1. General appearance of the microstructure. notice the random distribution of the martensite plates. 
mains grew at the expense of others. This observation and the observation that the lattice invariant deformation is provided by twrnning suggest that the martensite deforms by twinning at least in the early stages of deformation. Further, since the structure is ordered, deformation by motion of the dislocations would be difficult since it requires high stress to create the large areas of stacking faults that are associated with dislocation motion. No evidence for the presence of dislocations or twins in the surrounding matrix was observed and this suggests that the matrix (high temperature phase) is highly elastic. Therefore, the transformation distortion should be accommodated by the matrix only elastically.

To correlate the microstructure with the memory effect, specimens given various heat treatments were compared. Figure 2 is an example. The specimen in (a) was annealed at $300^{\circ} \mathrm{C}$ for 24 hours after cold rolling ( $\sim 50 \%$ reduction) and then quenched in iced water. It can be seen that the dislocation density was relatively high (these dislocations were introduced during the severe cold rolling). This specimen was very hard and stiff and it had negligible memory. In (b) the specimen was annealed at $600^{\circ} \mathrm{C}$ for 24 hours. It can be seen that the structure was relatively clear of dislocations. This specimen was very soft and it had good memory. This observation suggests that the dislocations already present will impede the motion of twin boundaries. This is probably why the specimen in (a) was very hard.

Since the twinning shear occurs only along a certain direction (in contrast to slip) the strain consumed in growing a twin will be regained when the twin shrinks. Accordingly, when the reverse transformation occurs and the twins (whether those associated with the transformation or those introduced mechanically) annihilate, the strain associated with these twins will be recovered. When a stress that is sufficient to initiate dislocation motion is applied, a decrease in the memory is expected since the strain associated with the motion of these dislocations is not reversible. This may explain the presence of a critical strain in the neighborhood of $6-7 \%$.

\section{THERMODYNAMICS}

A rigorous theory of the thermodynamic properties Nitinol ${ }^{4}$ explains the stress-strain temperature relationship of the metal in terms of an equilibrium between the cubic and orthorhombic forms. Under applied stress, the transition between the phases is first order, as is evidenced by the flat portions shown on the "idealized" stress-strain curves of Fig. 3, which indicate regions of coexistence of the two phases. Depending on the direction, this process is accompanied by the emission or absorption of latent heat. As the equilibrium stress is lowered by lowering the temperature, the flat portion of the stress-strain curve decreases in extent, and finally vanishes at zero stress. At this zero stress-equilibrium point (critical point) the transition is higher order and no latent heat is involved. This is why the transition has until now generally been considered to be second order. This theory seems to be consistent with all available experimental
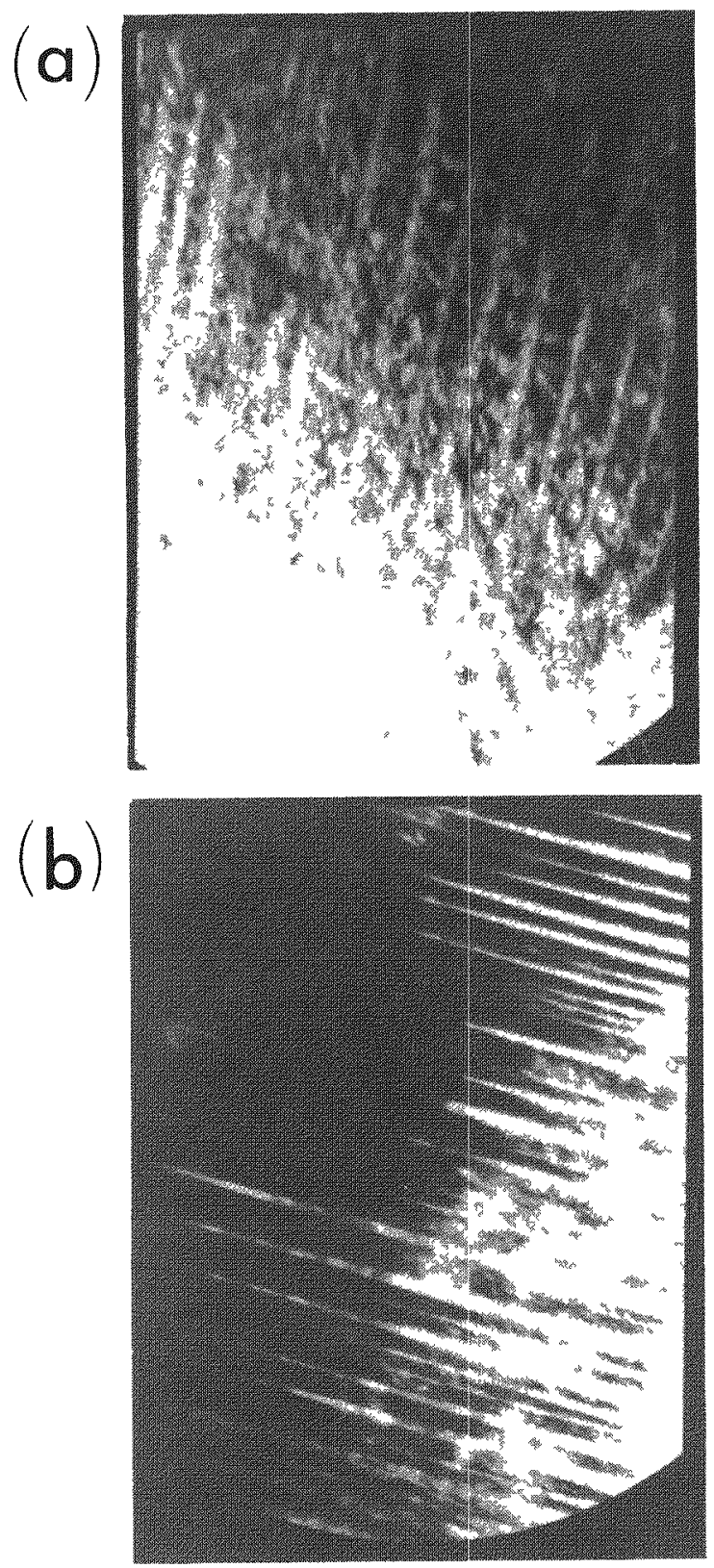

Fig. 2. Effect of thermal history on the microstructure.

(a) Specimen amnealed at $300^{\circ} \mathrm{C}$ for 24 hours and quenched in iced water. Notice the high density of disolocations.

(b) Specimen annealed at $600^{\circ} \mathrm{C}$ for 24 hours. Notice that the structure is almost free of dislocations.

(XBB 751-553)

data, and has the virtue of allowing engine cycle thermodynamics to be rigorously analyzed.

In Fig. 3 we have sketched two possible engine cycles, one of which is a Carnot cycle. reality, a Nitinol Carnot cycle would not be ve practical because of the rather small arca and inconvenient shape. However, the fact that it is conceivable is extremely important. It signifies that the potential efficiency of Nitinol engines is limited only by the absolute thermodynamic 


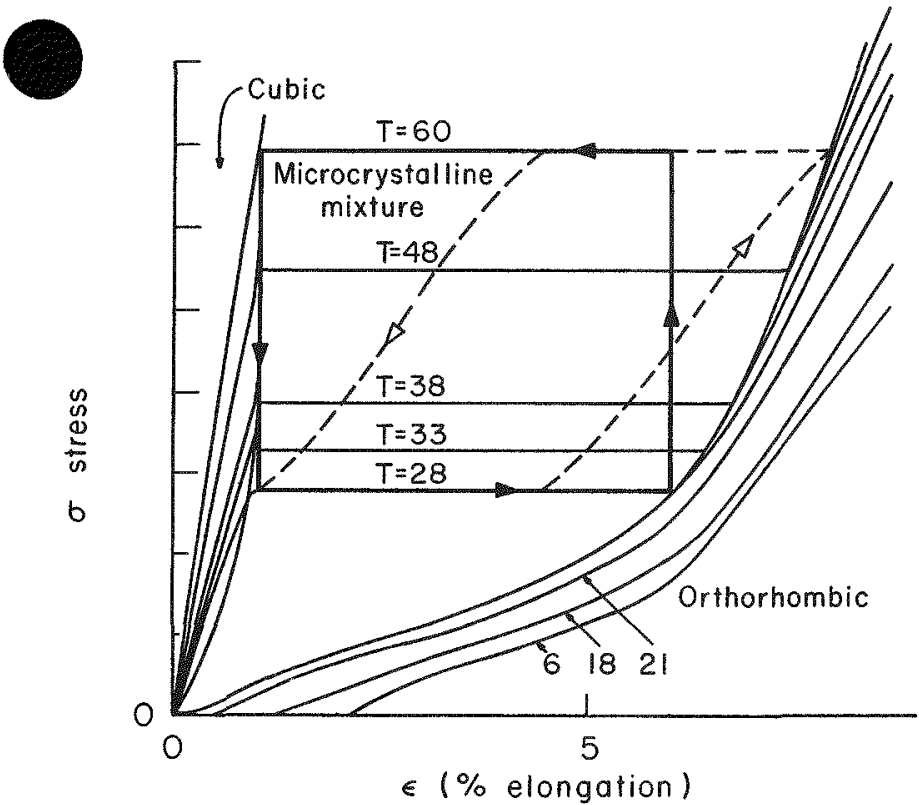

Fig. 3. Idealized stress-strain diagram. These stress-strain curves are based on experiment, but have been "idealized" with a theorist's touch. They are meant to illustrate qualitatively the properties of an "idea1" or "perfect" specimen which, unfortunately, does not yet exist. Two possible engine cycles are indicated. The one outlined with the dotted line is a typical Carnot cycle.

(XBL 751-2033)

Carnot Iimit. The strong dependence of equilibrium stress on temperature and the large "working strain" are what make the shape memory effect genuinely practical for heat engines while the superficially similar thermal expansion and thermoelastic effects (in other materials) are not.

\section{ENGINE DEVELOPMENT}

Test Bed Engine

The major effort has been placed on the construction of a linear-tension test-bed that could be converted into a $1-\mathrm{kW}$ power module. The test bed incorporates $70 \mathrm{~cm}$ long Nitinol wire elements (multiplicities of $0.5 \mathrm{~mm}$ diameter wires) in tension, which are supported between two non-parallel disks or "wobble-plates." Synchronous rotation of these plates increases the distance between corresponding points of attachment on their perimeters during one half revolution (the cold-deform side of the engine cycle), and decreases the distance between them during the other (hot) half revolution. During the hot cycle the wires are sprayed with hot water which causes them to shorten and forcefully advance the rotation of the system (seek the least energy position between the nonparallel plates). The angle between the wobble ates is adjustable permitting elongation of the tinol between the 1 imits of 0 to $7.0 \%$. The test bed has been provided with a direct readout of force and strain (work) developed in a particular working element by means of electronic instrumentation. Torque output can also be measured by means of a Prony brake. The test bed can be operated as an engine or power can be supplied to the machine by means of an electric motor to facilitate study of wire elements as they go through the heatingcooling cycle (Fig. 4). The work per cycle obtained in preliminary measurements at $3 \%$ elongation has been above 500 joules $/ \mathrm{kg}$ consistently. Creep and fatigue tests are planned and are needed to prove the feasibility of continuously operating Nitinol heat engines at high elongations.

\section{Continuous Band Engines}

A series of engines that directly utilize the stress differential between hot and cold Nitinol wires is also being developed. Successful prototypes based on this principle of a few watts output have been demonstrated. This concept enploys two coupled drive pulleys of differing diameters over which a continuous wire passes. Idler pulleys transport the wires into hot and cold baths and a torque differential is developed which results in a continuous translation of of wire over the system of pulleys. Continuous band engines have been operated with a single Nitinol wire 1oop in tension and others with a Nitinol wire helical spring formed into a continuous 1oop. Efficiency of these engines is jmproved by incorporating a regenerative cycle. The Nitinol element passes through a tube as it travels between the hot and
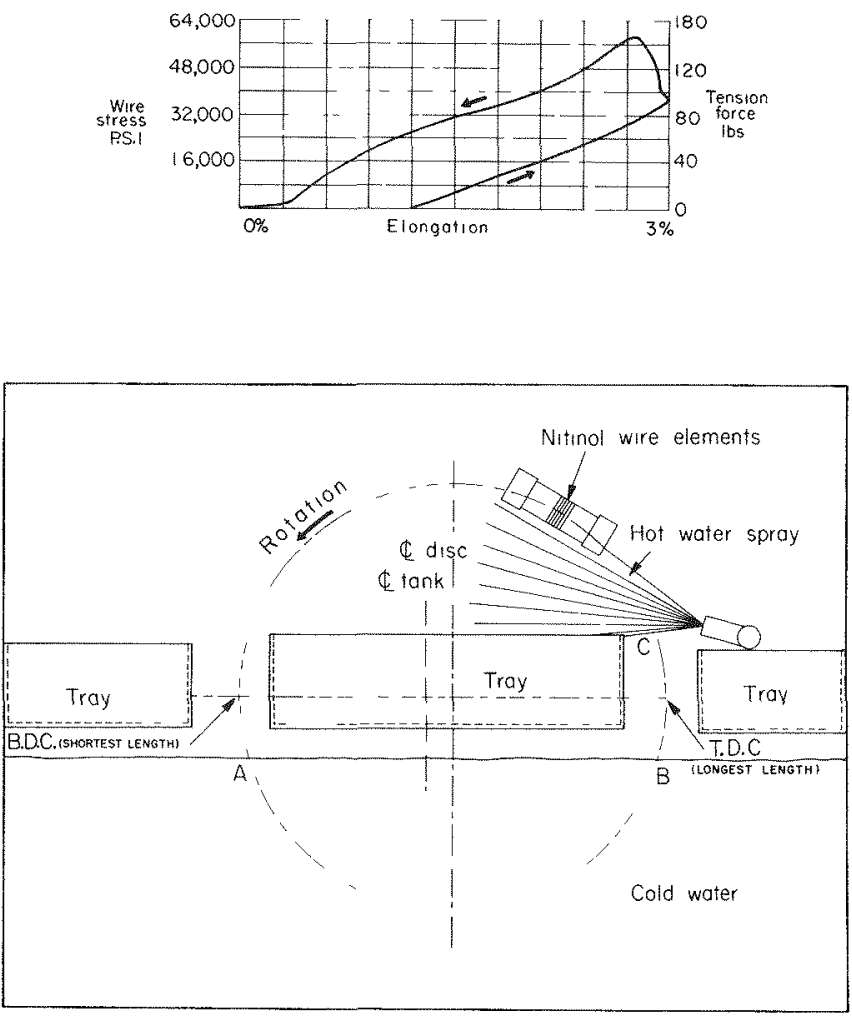

Fig. 4. Nitinol test bed. "Wobble plate" test bed. Only one test Nitinol element is shown. Heated Nitinol in its high strength cubic state contracts rotating the drive shafts to the minimum energy position. Simultaneously, the opposite cooled Nitinol element (not shown) is strained in its low strength orthorhombic state. The measured stress-strain behavior of a Nitinol element is shown above.

(XBL 751-2051) 
cold pulleys and again on its return exchanging the sensible heat. Measurement of engine efficiencies will result from calorimetric studies. To increase the power of this type of engine, we have begun construction of a woven belt-1ike band containing Nitinol.

\section{OTHER RESEARCH}

Nitinol has also been studied through computer simulation of engine cycling and by stressstrain measurements. The computer program is capable of varying such parameters as elongation,

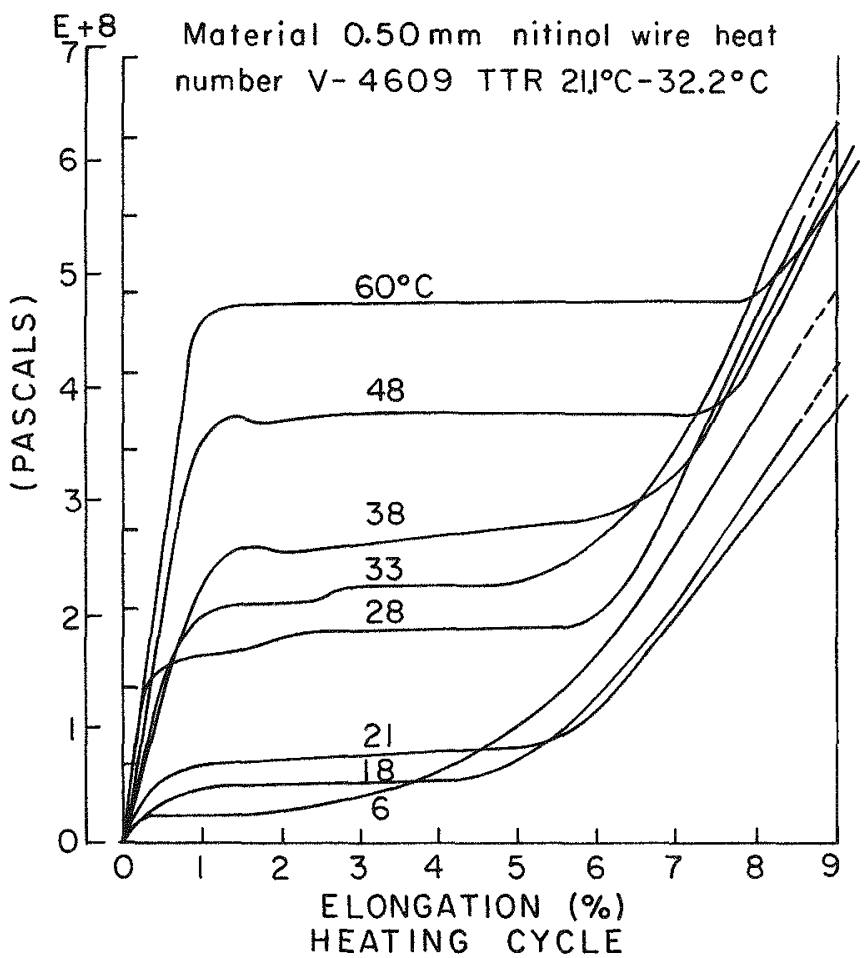

Fig. 5. Stress-strain diagram. Test data reported in References 5 and 6. 6894 PASCALS $=1 \mathrm{lb} / \mathrm{in}^{2}$.

(XBL $7411-8230)$ cooling/heating phase angle, and the various temperatures. Computer parametric studies have been effective in identifying the sensitive parameters and test bed engine settings. Stress-strain tensile tests have been performed 5,6 that confirm the published data of other investigators and characterize the materials currently being incorporated in LBL prototypes (Fig. 5). Further metallurgical studies include the effects of various welding and annealing techniques on the crystal structure and performance of the $\mathrm{Ni}-\mathrm{Ti}$ material.

\section{FOOTNOTES AND REFERENCES}

ork supported by the National Science FoundationRANN Division and the U.S.E.R.D.A.

1. Proposal for Application of Solid State Energy Conversion to Cooling of Buildings, Lawrence Berkeley Laboratory, Energy and Environment Division, submitted to the National Science Foundation (RANN), Nov. 1973. NSF Grant AG-550, Proposal No. P416452. Prototype is U.S. Patent \#3,913,326. Ridgway M. Banks assignor to the United States of America.

2. U. S. Patent No. 3, 403, 238; Conversion of Heat Energy to Mechanical Energy, S. J. Buehler et al., assignors to the United States of America. The alloy takes its name from Nicke1, Titanium, and Naval Ordnance Laboratory. The prefix "55" refers to the nominal percentage by weight of $\mathrm{Ni}$ in the compound.

3. C. M. Jackson, H. J. Wagner, and R. J. Wasilewski, 55-Nitinol-The Alloy with a MemoryNASA Report SP-5110, NASA (Washington, D.C.), 1972, pp. 65-70.

4. 0 . Weres, "The Thermodynamics of Nitinol", Internal Communication, LBL, Dec. 1974.

5. D. Ploeger, Nitinol Wire Testing Program, LBL Eng. Note M4778 (UCID 3669), Nov. 1974).

6. D. Ploeger, Nitinol Wire Testing Program, Techniques and Procedures, LBL Eng. Note M4784 (UCID 3702).

\section{STUDIES OF CELLULOSE AS A CHEMTCAL AND ENERGY RESOURCE*}

Charles R. Wilke, Paul Carroad, Gerald R. Cysewski, Malcolm Evett, Raymond Freitas, Gautam Mitra, Beverly Rubik, Albert Sciamenna, Yoshio Yamanaka, R. D. Yang

\section{INTRODUCTION}

Cellulose, in combination with lesser quantities of other polysaccharides and 1ignin, is the major structural constituent of al1 plants. These substances represent a vast source of stored chemical energy that has been obtained from the sun by photosynthesis. A portion of this energy can be made available in convenient form by biochemical conversion of cellulose to energy-rich compounds. Conversion of the non-cellulosic constituents offers further potential. A particularly promising pathway for cellulose utilization is its comversion to glucose and other reducing sugars by enzymatic hydrolysis and then to ethyl alcohol or other products by fermentation. Glucose also can be used directly as food for humans and animals or indirectly as a raw material for microbial protein. Alcohol is an excellent motor fuel. Ethylene can be made by dehydration of alcohol. In terms of available energy expressed either as the heat of combustion of cellulose, or of the glucose or alcohol theoretically obtained from it, a pound of cellulose is roughly equivalent to $0.35 \mathrm{lb}$. of gasoline $(7200 \mathrm{Btu})$.

While limitations of technology and curre economic factors preclude direct utilization of most cellulose as an energy resource, certain sources of cellulose do appear within range of current technology for economic processing. Two hundred ninty million tons per year of residential, 
institutional, and commercial solid wastes conining approximately $50 \%$ paper or other cellulosic terials are produced annually in the United States. In addition, there are on the order of 115 million tons of cellulosic wastes from wood manufacturing and sugar cane. The necessity for collection and disposal of such wastes provides a potential opportunity for hydrolysis of the cellulose fraction.

As a longer range source, there are produced in the U.S. approximately two billion tons per year of agricultural wastes which have a high cellulose content. planned coordination of food production with cellulosic processing could be an effective way of utilizing a part of this material. In case of sufficient need, faming might be conducted specifically to provide cellulose.

As hydrocarbon resources becomc depleted, a new chemical-energy industry based on cellulose can be visualized as one of the alternatives.

\section{A PROPOSED PROCESS FOR ENZYMATIC HYDROLYSTS OF CELLULOSE}

\section{Background}

Hydrolysis of cellulose theoretically produces glucose according to the reaction:

$$
\left(\mathrm{C}_{6} \mathrm{H}_{10} \mathrm{O}_{5}\right)_{\mathrm{X}}+\mathrm{X} \mathrm{H}_{2} \mathrm{O} \rightarrow \mathrm{X} \mathrm{C}_{6} \mathrm{H}_{12}{ }_{6}{ }_{6} .
$$

Mineral acids or enzymes in aqueous solution can serve as catalysts. Hydrolysis of the polysaccharides of wood yields, in addition to glucose, generally minor quantities of other reducing sugars including mannose, galactose, fructose, xylose, and aribinose. Reducing sugars are of particular interest as a potential carbon and energy source for fermentation processes.

Most early efforts to produce sugars from wood employed acid hydrolysis, the most notable being the Bergius process with hydrochloric acid in the $1930^{\prime} \mathrm{s}$. The acid processes have not proved economically feasible because of acid losses, costly materials needed in construction, and corrosion.

In recent years interest has developed in enzymatic hydrolysis, particularly through the efforts of Reese, Mandels, and others at the U.S. Army Natick Laboratories. Through extensive screening and mutation, organisms have been found that produce enzymes that are highly effective in hydrolyzing cellulose under non-corrosive conditions. Over the past several years in our program at Berkeley we have expanded upon the basic findings of the Natick group to develop several original processing procedures that are incorporated in a tentative new process for cellulose hydrolysis, - iefly described below. Additional details are ilable in recent publications. 1-3

\section{Process Description}

A preliminary design has been prepared for processing paper waste with enzymes produced by the fungus Trichoderma viride QM 9414, a mutant developed by the Natick group (see Fig. 1) The feed stock is assumed to contain $61 \%$ cellulose of which $52 \%$ is converted to glucose by digestion with aqueous enzyme solution. The flow diagram (Fig. 2) indicates the major processing steps for a typical operation. Facilitics for feed preparation and combustion of the residual solids are not shown.

Waste paper at a rate of 833 tons per day (dry basis), 6\% moisture content, is passed through a shredder, milled to -20 mesh and fed through an adsorption system to the hydrolys is vessels. In the hydrolysis system the ground paper at a concentration of $5 \%$ wt. is held for 40 hours with aqueous enzyme solution.

Fresh make-up enzyme is produced by the fungus in a two-stage formentation system. Rapid cell growth on a medium consisting of superphosphate, Proflo o11, and flucose plus minor minerals occurs in the first stage. In the second stage plant feed solids are added to induce enzyme synthesis. A recycle stream of cells to the induction vessels increases the productivity. The cells and undigested cellulose are removed from the solution by a pressure filter.

The hydrolyzer effluent is passed through a pre-coat rotary vacuum filter from which the residual solids are fed to a furnace to provide distillation heat and steam and the solution is fed to the enzyme recovery section. Approximately $95 \%$ of the enzyme in the sugar solution is removed in three adsorption stages. A small portion of the glucose solution is retumed to the first fermentation stage and the remainder comprises the final plant production.

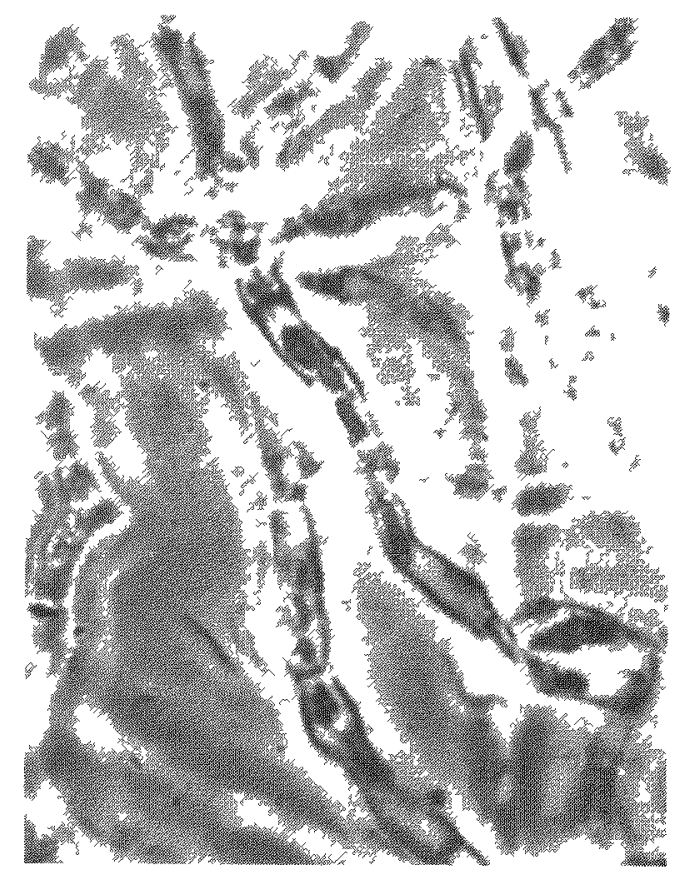

Fig. 1. Microphotograph of $T$. viride fungus which produces cellulase enzyme. The mycelium is approximately 1 micron in diameter.
(XBB 744-2517) 


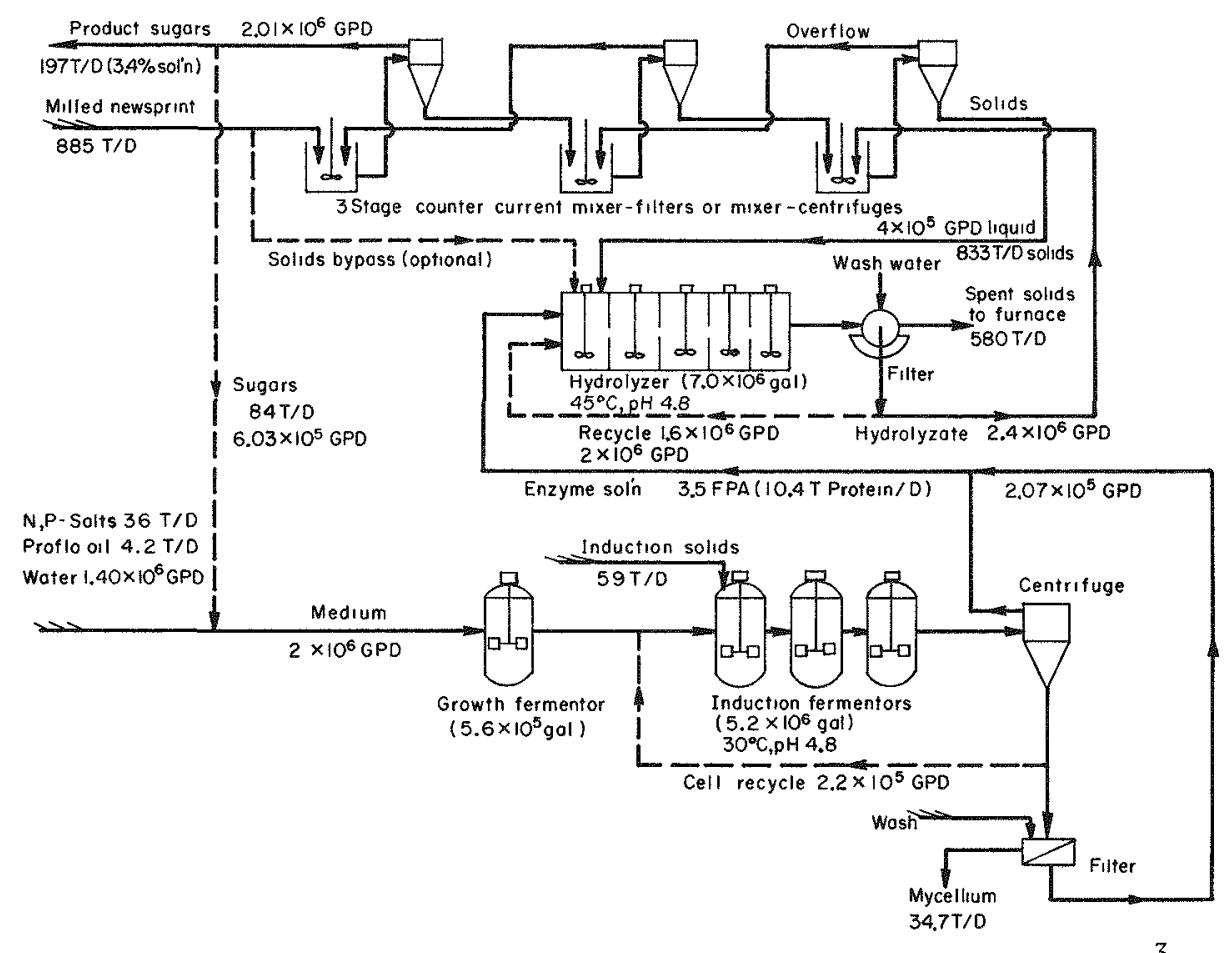

Fig. 2. Process flow diagram of enzymatic hydrolysis of newsprint. ${ }^{3}$

(XBL 752-2392)

A very preliminary cost analysis indicates that it may be possible to produce sugars in this manner at a cost competitive with alternate sugar sources. However, additional basic research is needed to verify some of the major assumptions of the present process and to seek further improvement. It will also be desirable to test these processing methods in a pilot plant operation on typical cellulosic materials.

\section{ALCOHOL FERMENTATION STUDIES}

One of the more promising uses of the sugars produced by enzymatic hydrolysis is their fermentation to ethyl alcohol. Approximately one million tons per year of industrial alcohol are produced annually in the U. S., largely from petrochemical raw materials. A study of alcohol fermentation has been initiated in an effort to assess the feasibility of using the hydrolysis product and to seek possible improvement in the existing technology for alcohol production. A novel technique of fermentation under vacuum is currently under development. This method avoids product inhibition by boiling the alcohol directly off the yeast suspension under low pressure as the fermentation proceeds. Preliminary engineering analysis, based on limited data from our 1aboratory, indicates that significant economies in cost and energy consumption may be possible with the vacuum operation. Further laboratory work leading to a pilot plant study is in progress.

\section{CURRENT FUNDAMENTAL STUDIES}

In addition to continued development of the cellulose hydrolysis and alcohol fermentation processes described above, a general program of re- lated fundamental studies is under way. Hopefully, these studies will increase our fundamental knowledge of microbial and enzymatic processes and lead to new and improved concepts for utilization of cellulose. Among projects that are currently in progress or planned for the near tuture, are (1) a study of the enzymatic degradation of lignin as a possible aid to cellulose hydrolysis,

(2) a study of the use of $\beta$ glucosidase, an enzyme that converts cellobiose to glucose, to improve the rate of hydrolysis and to increase the yield of glucose, (3) use of dense cell cultures to improve the productivity of microbial reactors, (4) utilization of hemi-cellulose, (5) study of chemical pretreatment of cellulosic materials as an alternative to milling, (6) a study of acid hydrolysis in comparison with the enzymatic method, (7) investigation of new sources of enzymes for cellulose hydrolysis and (8) evaluation of various alternative sources of cellulose with respect to their supply, cost and susceptibility to hydrolysis.

\section{FOOTNOTE AND REFERENCES}

"Work supported by the National Science Foundation-

International Bilateral Program and the U.S.E.R.D.A.

1. G. Mitra, and C. R. Wilke, "Continuous Cellulase Production," Biotechnology and Bioengineering, John Wiley \& Sons, N.Y., 171 (1975).

2. G. Mitra, and C. R. Wilke,"Enzymatic Utilizat of Waste Cellulosics," LBL-2334 (Revised) January 1975.

3. C. R. Wilke, and R. D. Yang, "Process Development and Design Studies for the Enzymatic Hydrolysis of Cellulose, LBL-3254, Apri1, 1975. 
This project is concerned with the measurement of the flux of energy from the sun and from the circumsolar region (the small-angle region around the sun) as a function of angle, wavelength, and atmospheric conditions. These measurements will provide accurate predictions of the performance of solar thermal conversion systems using focusing collectors.

Current and proposed research projects on the conversion of solar energy to thermal energy, usually for the generation of electricity, genera11y involve focusing mirrors of lenses to concentrate the incident energy on a receiver. The use of such collectors results in high-temperature operation of the receiver, in turn allowing a conversion efficiency from heat to electricity comparable to that of conventional generating stations.

Presently, calculations of the expected system performance of ground-based focusing collectors assume that the solar flux is contained within the $1 / 2^{\circ}$ angle subtended by the sun. This assumption would be justified if the only atmospheric effect were absorption of sunlight. However, such is not the case. The sunlight is also scattered by molecules (Rayleigh scattering) and by suspended particles and aerosols (Mie scattering). While the molecular scattering is nearly isotropic, the particles and aerosols scatter sunlight predominantly through small angles, resulting in circumsolar radiation (also referred to as the solar aureole). Pyrheliometers, the standard instruments used to measure the "direct" solar flux, typically have a $6^{\circ}$ field of view. Thus circumsolar radiation is included in the measurement of the pyrheliometers, overestimating the amount of energy that would be collected by focusing devices.

Since accurate measurements of the circumsolar/solar intensity ratio are not available, this project has undertaken an effort to provide data on solar and circumsolar radiation through the development of suitable instrumentation and a comprehensive measurement program. For that phase of the project reported here, the objectives were to design, construct and test an instrument with: (1) the ability to determine the spatial (angular) distribution of the solar and circumsolar radiation with sufficient resolution and accuracy that the determination may be usefully applied to performance calculations of solar thermal conversion systems, and (2) the ability to measure the amount of circumsolar radiation between the edge of the sun's disc and a $3^{\circ}$ radius circle, and thus the adequacy of existing pyrheliometers as measurers of the "direct" solar radiation.

A number of instrumental design criteria were established to meet these objectives. The instrument had to be able to measure the angular stribution of the solar and circumsolar radiation thin a field of view of $6^{\circ}$ (corresponding to that of typical pyrheliometers) centered on the sun. A basic angular resolution of $\sim 1 / 10$ the diameter of the sun was required to permit adequate measurements near the edge of the sun. Circumsolar radiation from the edge of the sun out to $3^{\circ}$ from the center had to be measurable when its integractu intensity was $1 \%$ or more of that coming from the sun's disc. This criterion set limits on the amount of direct sunlight that could be scattered from the optical and non-optical elements of the telescope. To make satisfactory measurements over the entire solar spectrum, the instrument had to be able to make nearly wavelength-independent measurements. For determination of the wavelength dependence of circumsolar radiation, measurements using broadband filters were deemed adequate.

The instrument was designed for absolute calibration by making simultaneous measurements with a pyrheliometer and equating the latter's measurement to the integral (over the field of view of the pyrheliometer) of the instrument's measurement. Other requirements were that the instrument system be transportable in a sma11 van type vehicle, that the data be recorded in a form suitable for computer processing, and that the system be capable of unattended operation during the course of a day.

The resulting instrument system consists of a "scanning telescope", designed and fabricated at LBL, some conventional solar instruments, and a digital electronics control system. The design of the scanning telescope is presented in Fig. 1.

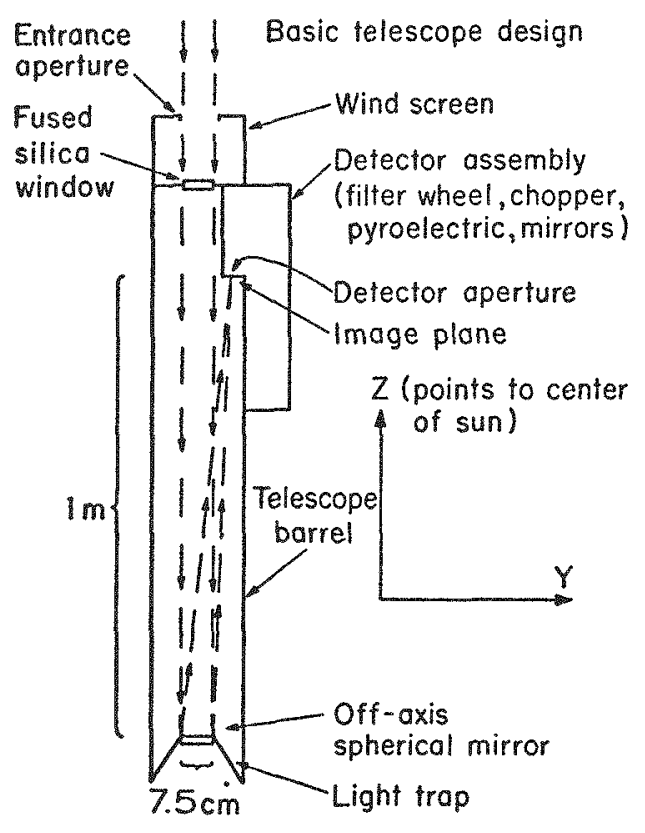

Fig. 1. Design of the scanning telescope for measuring circumsolar radiation. The basic optical element is an off axis mirror of $7.5 \mathrm{~cm}$ diameter and one meter focal length. The mirror forms an image of the sun and adjacent sky on a plate off to the side of the incoming 1ight. A small hole in this plate, the detector aperture, defines the angular resolution. The amount of 1 ight passing through the aperture into the detector assembly constitutes the fundamental measurement.

(XBL 749-4243) 
The basic optical element is an off-axis mirror of $7.5 \mathrm{~cm}$ diameter and one meter focal length. A fused silica window protects the mirror from the environment. The mirror forms an image of the sun and adjacent sky on a plate off to the side of the incoming light. A small hole in this plate, the detector aperature, defines the angular resolution $(1 / 20$ the solar diameter), and the amount of light passing through the aperture into the detector assembly constitutes the fundamental measurement. In the detector assembly, the light is mechanically chopped, optically filtered, and focused onto a pyroelectric detector, a type of thermal detector. Included in a complete set of data are one wavelength-independent (no filter) measurement and eight wavelength-dependent measurements, using filters that divide the solar spectrum into eight bands of roughly equal energy content.

As illustrated in Fig. 2, the telescope is mounted on a solar tracker that keeps a reference platform pointed at the center of the sun. The angular measurements are made by rotating the telescope barrel through a $6^{\circ}$ angle about a pivot point on the platform. An active cavity radiometer, a type of pyrheliometer that is self-calibrated in electrical units, 1 was acquired to provide the calibration for the scanning telescope, as well as the usual "normal incidence" measurement. Other measures of the solar radiation are provided by two pyranometers ${ }^{2}$ (one mounted in the usual horizontal. position and one tracking the sun), and by a sun photometer. 3

The operation of the instrument is controlled by a digital electronics system. This control system drives the various mechanical motions, synchronizes and digitizes the various analog output signals from the detectors, and records the data on magnetic tape.

Preliminary tests with the scanning telescope have been directed toward meeting the most difficult criterion: the ability to measure circumsolar radiation at intensity levels less than $1 \%$ of that coming from the disc of the sun. Figure 3 shows a measurement for a clear day and, for comparison, a day with light haze. The clear day measurement gave a circumsolar/solar integrated intensity ratio of $0.28 \%$, comfortably below the requirement. Whether this measurement represented atmospheric scattering or scattering within the instrument itself has not yet been determined. Work during the next phase of the project may wel1 lower yet further the 1 imit of circumsolar levels that are measurable with this instrument.

Now that the telescope has been fabricated and has undergone preliminary testing, the next phase of the project will include evaluation of the instrument system during a measurement program in the San Francisco Bay Area. It will then be used in other geographic areas, under a variety of atmospheric conditions. Three additional systems will be constructed for deployment at potential sites for future solar thermal conversion installations.
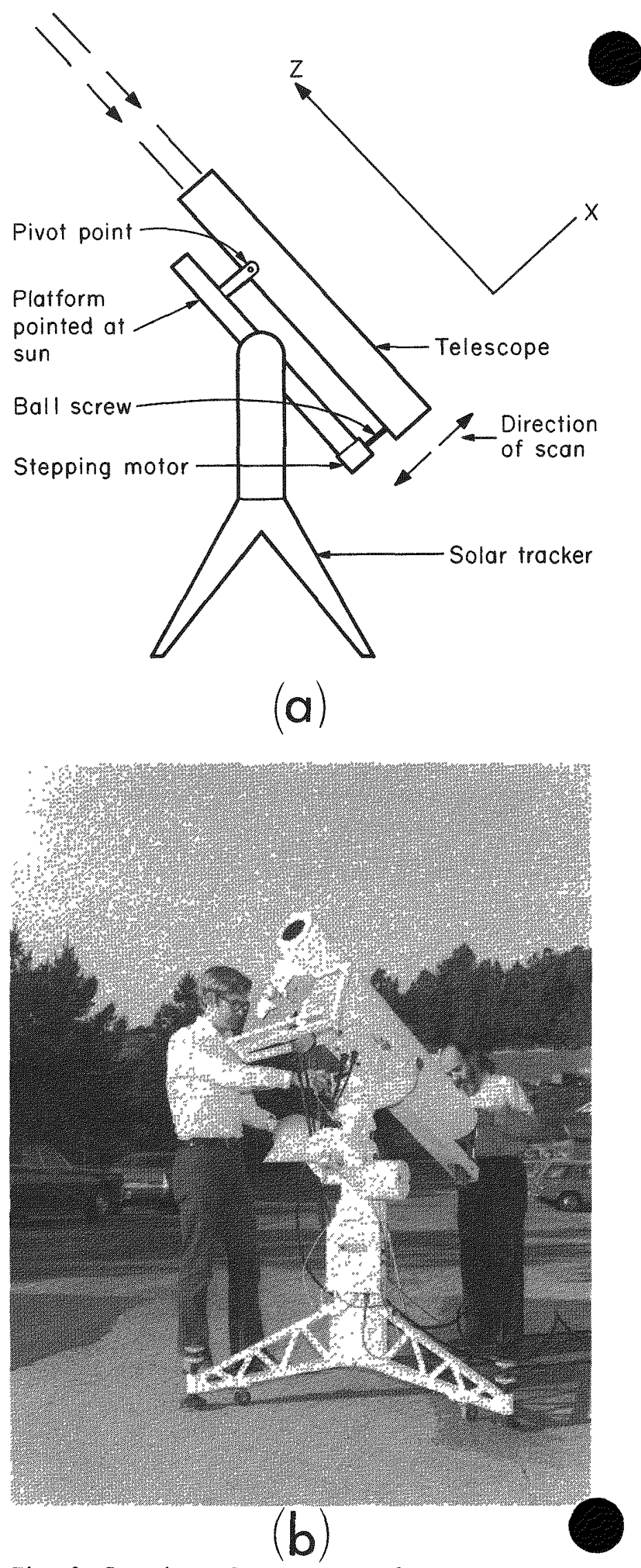

Fig. 2. Scanning telescope mounted on a solar tracker: (a) schematic and (b) photograph.

(XBL 749-4244, CBB 7412-8879) 


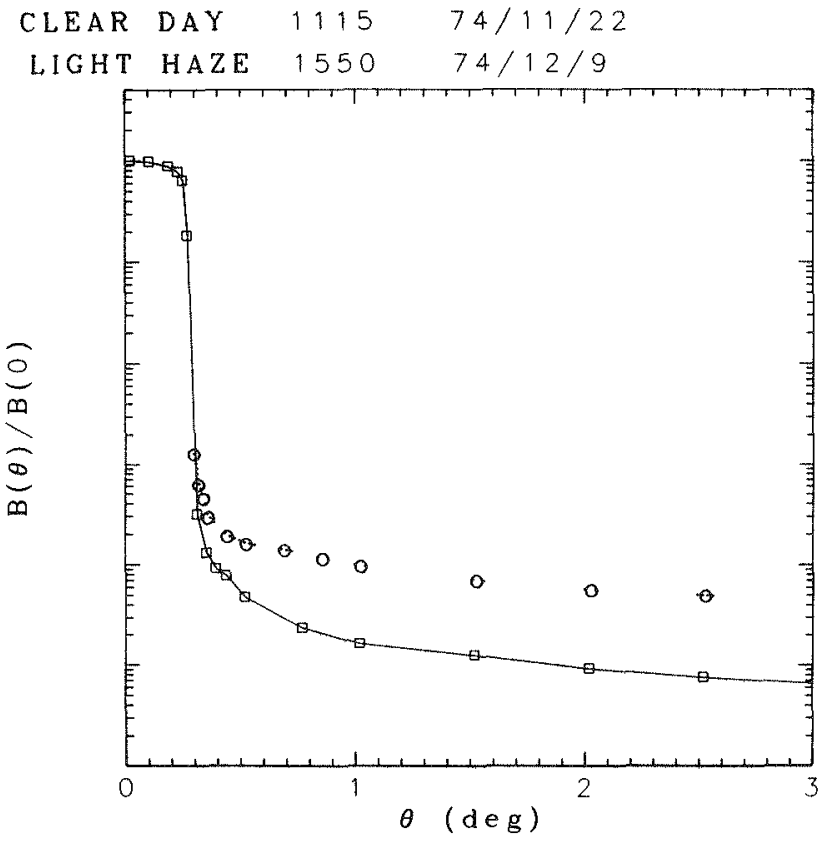

\section{FOOTNOTES AND REFERENCES}

${ }^{*}$ Condensed from 'Measurement of Circumsolar Radiation, Progress Report No. 1" UCID-3705, January, 1975.

Tork supported by the National Science Foundation - RANN Division.

1. R. C. Wi11son, App. Opt. 12, 810 (1973).

2. D. J. Norris, Solar Energy 14, 99 (1973).

3. E. C. Flowers, R. A. McCormick, and K. R. Kurfis, J. App1. Meteor. 8, 955 (1969).

Fig. 3. Results from preliminary tests with the scanning telescope. The relative intensity of the uncident radiation is measured as a function of the angle from the center of the sun. The clear day circumsolar/solar integrated intensity ratio is $0.28 \%$ and the light haze day ratio is $1.2 \%$. 


\title{
Instrumentation and Measurement
}

\author{
INTRODUCTION
}

Energy production and its utilization result in the emission of contaminants that may be harmful to man and many of his activities. It is therefore necessary that means be provided to measure the contaminants produced, to understand their behavior including their chemistry, and to determine their effects, particularly on health but also on other human activities such as agriculture and fisheries. In order to acquire understanding of and control of harmful contaminants, adequate methods to monitor the level of various substances (often present at low concentrations) are required. The Energy and Environment Division's program on instrumentation and measurement is directed towards meeting these needs.

An emphasis of the program is on the development of physical methods as opposed to conventional chemical analysis techniques. The advantages of physical methods, now widely recognized, include ease of operation and minimal sample preparation. In some cases physical methods can provide multi- parameter measurements, and frequently they provide the only means of attaining the sensitivity necessary for the detection of trace contaminants.

The program includes research on and development of both instrumentation and analytical procedures. Among the topics now being investigated in instrumentation are microwave spectroscopy, materials for radiation detectors, $x$-ray fluorescence spectroscopy, resonance Raman spectroscopy, liquid crystal detectors, atomic absorption spectroscopy, and electroanalytical chemistry.

While environmental problems provide the central focus for our work on instrumentation and measurement, we are also working in other areas that are relevant to energy production and use. Two of these areas are combustion research and the geosciences (especially geochemical exploration). Work in these areas has only recently been initiated; future anmual reports can be expected to contain more on these topics.

\section{THE SURVEY OF INSTRUMENTATION FOR ENVIRONMENTAL MONITORING**}

D. A. Mack, N. M. Amer, R. J. Budnitz, R. M. Graven,

C. C. Hollowel1, M. J. Kland, R. A. McLaughlin, W. D. Macleod, G. A. Morton, S. L. Phillips, J.A. Stokes

It is essential to the Nation's program of environmental improvement that accurate and efficient measurements be made of the parameters affecting the environment. To aid in this task, the Division's Environmental Instrumentation Group has been conducting and updating a comprehensive Survey of Instrumentation for Environmental Monitoring since 1971.

The Survey is a critical in-depth examination of instrumentation suitable for monitoring important pollutants and parameters in the environment. It is organized into four sections, which cover air, water, radiation, and biomedical instrumentation. Each section is published in a large loose-leaf binder (the Air section now fills two such binders) to facilitate periodic revision. Table 1 1ists some of the topics covered in the Survey.

For each parameter or pollutant studied, the Survey has two parts: a review of the state of the art of monitoring, followed by Instrument Notes containing specifications and critical information about individual instruments, primarily those commercially-available.

In addition to filling "information gaps" by compiling and disseminating current information, the Survey makes it possible to examine and then recommend new methods of detection and analysis. Recommendations are also made for the exploration and development of techniques which are utilized in other disciplines and appear to be applicable to environmental problems.

At the present time, it is clear that in a number of areas the state-of-the-art of instrumentation for environmental monitoring is inadequate. For example, a great deal of commercially available equipment is sensitive to interferring parameters, analysis may be time-consuming or expensive, different chemical or physical forms of a pollutant may not be differentiated, and often sampling techniques are not wel1 developed. In many such cases, the Survey has made specific recommendations to alleviate the deficiencies.

At this time, the initial tasks of the Survey are essentially complete. Within the last year the issue of calibration of air and water instruments has been addressed in greater detail. During FY 76 , the Survey group will concentrate on the issue of sampling methods for air and water analysis along with its on-going task of up-dating the various sections of the Survey. This latter task is vital because of significant changes which are constant occurring as the result of new technology, new instruments, and new regulations and standards.

Over 3,500 volumes are now in use throughout the world. The wide acceptance of the Survey is due partly to the generous contributions of scores 
TABLE 1. Pollutants and parameters covered in Survey of Instrumentation for Environmental Monitoring.

\begin{tabular}{|c|c|c|c|}
\hline $\begin{array}{l}\text { Vo1. I } \\
\text { AIR }\end{array}$ & $\begin{array}{l}\text { Vol. II } \\
\text { WATER }\end{array}$ & $\begin{array}{l}\text { Vol. III } \\
\text { RADIATION }\end{array}$ & $\begin{array}{l}\text { Vol. IV } \\
\text { BIOMEDICAL }\end{array}$ \\
\hline $\mathrm{SO}_{2}$ & Meta1s & A1pha Radiation & $\mathrm{CO}$ \\
\hline $\mathrm{NO}$ and $\mathrm{NO}_{2}$ & Nitrogen & Beta Radiation & $\mathrm{SO}_{2}$ \\
\hline Oxidants & Phosphorus & $\begin{array}{l}X \text { and Ganma } \\
\text { Radiation }\end{array}$ & Asbestos \\
\hline $\mathrm{CO}$ & Sulfur & Gamma Spectroscopy & Mercury \\
\hline Hydrocarbons & $\begin{array}{l}\text { Biological Oxygen } \\
\text { Demand }\end{array}$ & Neutrons & Cadmium \\
\hline $\begin{array}{l}\text { Particulates } \\
\text { Mass } \\
\text { Size }\end{array}$ & $\begin{array}{l}\text { Chemical Oxygen } \\
\text { Demand }\end{array}$ & $\begin{array}{l}\text { Personne1 Dosimetry } \\
\text { Radionuclides }\end{array}$ & $\begin{array}{l}\text { Lead } \\
\text { Pesticides }\end{array}$ \\
\hline Opacity & Dissolved Oxygen & Tritrium & Radiation \\
\hline $\begin{array}{l}\text { Composition } \\
\text { Mercury }\end{array}$ & Total Organic Carbon & $\begin{array}{l}\text { Krypton- } 85 \\
\text { Strontium- } 89 \text { and }-90\end{array}$ & \\
\hline & Pesticides & Iodine-129 and -131 & \\
\hline Asbestos & Phenolics & $\begin{array}{l}\text { Radon- } 222 \text { and its } \\
\text { daughters }\end{array}$ & \\
\hline Lead & $\begin{array}{l}\text { Oi1 and Grease } \\
\mathrm{pH}\end{array}$ & $\begin{array}{l}\text { Radium } \\
\text { Uranium }\end{array}$ & \\
\hline Noise & Turbidity & $\begin{array}{l}\text { Plutonium } \\
\text { Microwaves }\end{array}$ & \\
\hline & Temperature & $\begin{array}{l}\text { Lasers } \\
\text { U1traviolet }\end{array}$ & \\
\hline
\end{tabular}

of experienced workers throughout the country. For example, the Radiation volume alone lists 79 persons who have made contributions to that phase of the Survey, mostly as expert reviewers of the various sections. A great many other scientists and engineers have contributed to the reviews of instruments described in the Instrument Notes.

\section{Footnote}

* Research supported by the National Science Foundation; Research Applied to National Needs.

\section{DETECTION AND MEASUREMENTS OF AIR POLLUTANTS BY MICROWAVE ROTATIONAL SPECTROSCOPY}

B. Leskovar, W. F. Kolbe, and D. B. Hopkins

The technique of microwave rotational spectroscopy offers a highly specific and sensitive experimental research method for studying chemical kinetics and monitoring gaseous pollutants in the environment. For this project a new high-sensitivity laboratory-type microwave spectrometer with multiparameter capabilities is under development. Our initial action was the investigation of the microwave frequency spectrum and pertinent absorption

efficient values of the sulfur dioxide polar lecule. Also, in order to establish design riteria, we have examined factors which cause the introduction of noise during the generation of microwave radiation.

Although the microwave spectrum of ${ }^{32} \mathrm{~S}^{16} \mathrm{O}_{2}$ has been extensively studied and compilations of measured and calculated frequencies have been made, calculations of the absorption coefficient values corresponding to these transitions are not available. Similarly, absorption coefficient values have been measured only for a limited number of transitions in a frequency range from 19.6 to $39.4 \mathrm{GHz} .{ }^{1}$ In order to obtain accurate and complete information, a computer program was written to calculate both the rotational transition frequencies and pertinent absorption coefficient values.

The microwave spectrum of ${ }^{32} \mathrm{~S}^{16} \mathrm{O}_{2}$ was calculated using the Kirchhoff formalism, which includes the Watson treatment of the molecular centrifugal distortion. 2 The Wang-transformed prolate symmetric 
rotor basis set was used and the resulting submatrices, obtained for each value of the rotational quantum number, $J$, were diagonalized exactly by computer.

Peak values of the absorption coefficient, $\gamma_{\text {max }}$, for each transition, were calculated from the modified Van Vleck-Weisskopf equation, 3

$$
\gamma_{\max }=\frac{8 \pi^{2} f_{0}^{2} \beta}{3 c k T} \frac{(2 J+1)}{Q_{r}}\left|\mu_{i j}\right|^{2}\left(\frac{N}{\Delta f}\right) e^{-W / k T},
$$

where $f_{0}$ is the resonant frequency, $\beta$ is the fraction of the molecules in the vibrational state involved, $\mathrm{k}$ is Boltzmann's constant, $\mathrm{T}$ is the absolute temperature, $J$ is the rotational quantum number, $\left|\mu_{i j}\right|^{2}$ is the square of the dipole moment matrix element for the transition considered, $N$ is the number of molecules per unit volune, $\Delta f$ is the absorption line half-width at half-maximum intensity, and $W$ is the energy of the lower state. For ${ }^{32} \mathrm{~S}^{16} \mathrm{O}_{2}$ the rotational partition function $\mathrm{Qr}_{\mathrm{r}}=(1 / \sigma)\left[\left(\pi /(\mathrm{ABC})(\mathrm{kT} / \mathrm{h})^{3}\right]^{\frac{1}{2}}\right.$, where $\mathrm{A}, \mathrm{B}$, and $\mathrm{C}$ are the rotational constants, and the symmetry number $\sigma=2$.

Absorption coefficient peak values were calculated for each transition using the direction cosine matrix elements derived by Schwendeman ${ }^{4}$ and the wave functions obtained by diagonalizing the energy matrices.

The transition frequencies and absorption coefficient values were calculated for all transitions up to $J=40$ that had a coefficient value greater than $10^{-10} \mathrm{~cm}^{-1}$ in the frequency range from 8 to $200 \mathrm{GHz}$. The results of the calculations are shown in Fig. 1 for frequencies up to $140 \mathrm{GHz}$. The comparison with available experimental data in the frequency region from 19.66 to $39.4 \mathrm{GHz}$ is also shown. The agreement between calculated values and experimentally obtained data is very good, particularly for frequencies above $26 \mathrm{GHz}$. However, additional measurements are necessary at frequencies above $40 \mathrm{GHz}$ and they will be made, particularly in the vicinity of $70 \mathrm{GHz}$, on the new spectrometer. Calculations show that the ${ }^{32} \mathrm{~S}^{16} \mathrm{O}_{2}$ transition at $68.9721 \mathrm{GHz}$, between the $6(0,6)$ and $6(1,5)$ levels, has a coefficient peak value of $\gamma_{\max }=4.3 \times 10^{-4} \mathrm{~cm}^{-1}$. This amount is a factor of ten greater than for any transition between 8 and $40 \mathrm{GHz}$.

Since the sensitivity of the tunable high-Q resonant cavity spectrometer is essentially determined by the total noise introduced during generation of the microwave radiation, the absorption process in the resonant cavity, and the detection and amplification of a spectral line signal, the spectrometer must be analyzed in detail with respect to various noise sources. The effective microwave source noise temperature was calculated according to the equation:

$$
\mathrm{T}_{\text {eff }}=\frac{\mathrm{P}_{0}}{2 \mathrm{k} \Delta \overrightarrow{\mathrm{B}}_{\mathrm{m}}}\left[\tau\left(\frac{\mathrm{N}_{\mathrm{AM}}}{\mathrm{C}}\right)_{\mathrm{DSB}}+\eta\left(\frac{\Delta \mathrm{f}_{\mathrm{rms}}}{\mathrm{f}_{\mathrm{m}}}\right)^{2}\right] \text {, }
$$

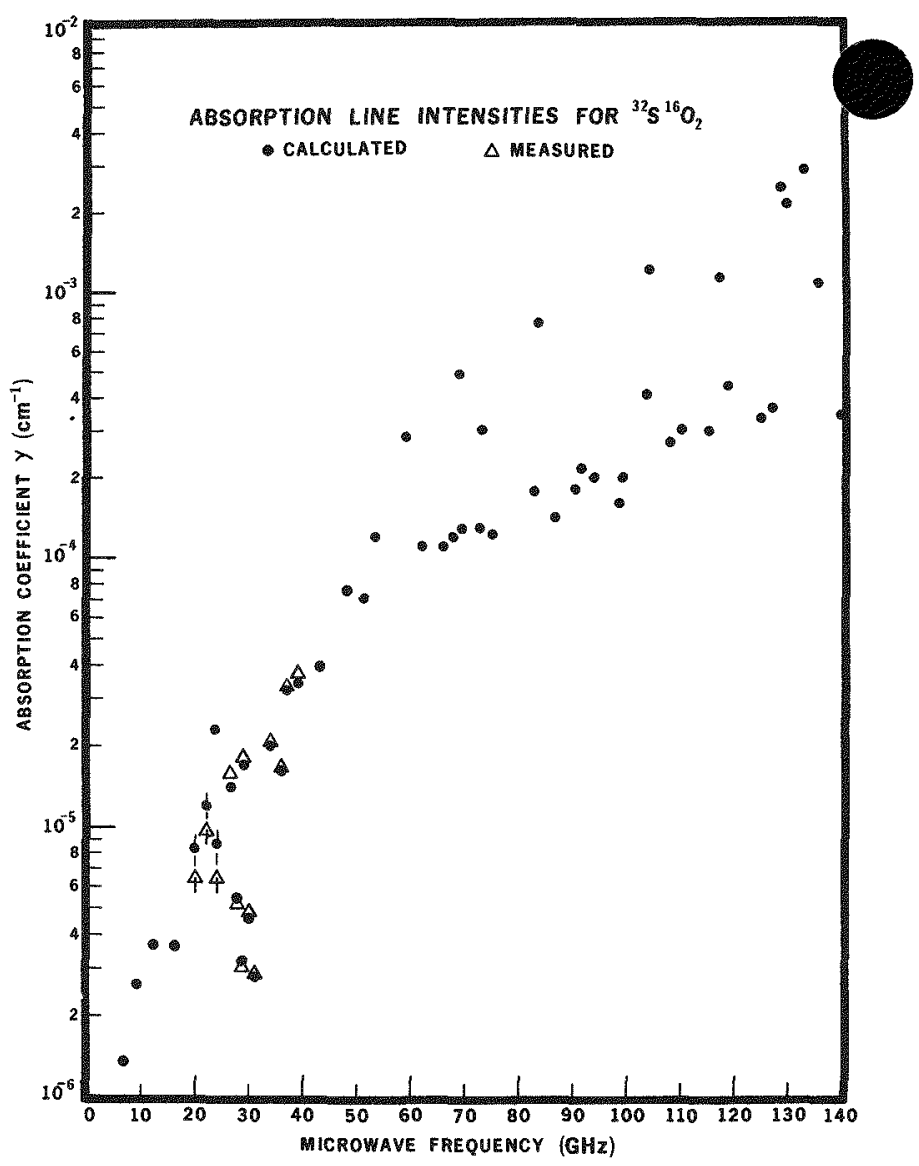

Fig. 1. Absorption line intensities for ${ }^{32} \mathrm{~S}^{16} \mathrm{O}_{2}$ as a function of frequency.

(XBL 751-86)

where $\mathrm{P}_{0}$ is the carrier power level, $\Delta \mathrm{B}_{\mathrm{m}}$ is the bandwidth over which $\left(\mathrm{N}_{\mathrm{AM}} / \mathrm{C}\right) \mathrm{DSB}$ and $\left(\Delta \mathrm{f}_{\mathrm{ms}} / f_{\mathrm{m}}\right)$ were orginally measured, ${ }^{\left(N_{A M}\right.}$ DSE $)_{D S B}$ is the ratio of double sideband AM noise power to carrier power in a bandwidth (BW) measured at a distance $f_{m}$ from the carrier, $\Delta f_{\mathrm{rms}}$ is the noisiness of the carrier, defined by the rms noise deviation which is measured in a bandwidth at the output of an FM discriminator with a microwave oscillator at its input, where $P_{0}$ is the carrier power leve1, $\Delta \mathrm{B}_{\mathrm{m}}$ is the bandwidth over which $\left(N_{A M} / C\right)$ DSB and $\left(\Delta f_{\text {rms }} / f_{m}\right)$ were originally measured, $\left(\mathrm{N}_{\mathrm{AM}} / \mathrm{C}\right) \mathrm{DSB}$ is the ratio of double sideband AM noise power to carrier power in a bandwidth (BW) measured at a distance $f_{m}$ from the carrier, $\Delta \mathrm{f}_{\mathrm{rms}}$ is the noisiness of the carrier, defined by the rms noise deviation which is measured in a bandwidth at the output of an FM discriminator with a microwave oscillator at its input, $f_{m}$ is the modulation frequency, and $\tau$ and $n$ are AM transmission and FM-AM conversion factors, respectively. 5 The transmission-factor $\tau=\Gamma_{0}^{2}+\left(Q_{0} f_{m} / F_{0}\right)^{2}$ is determined by the cavity resonant Irequency, $F_{0}$, and the factor, $\Gamma_{0}$, which depends upon the cavity coupling, as determined by the ratio of the external and unloaded $Q$ factors. The conversi factor $n=Q_{0}^{2} S^{2}+\phi^{2} \tau$ is determined by the longterm frequency stability $S$, the relative phase stability $\phi$ between the signal and reference wave, and the $Q_{0}$ and $\tau$ factors. The effective noise temperature, as a function of carrier frequency 


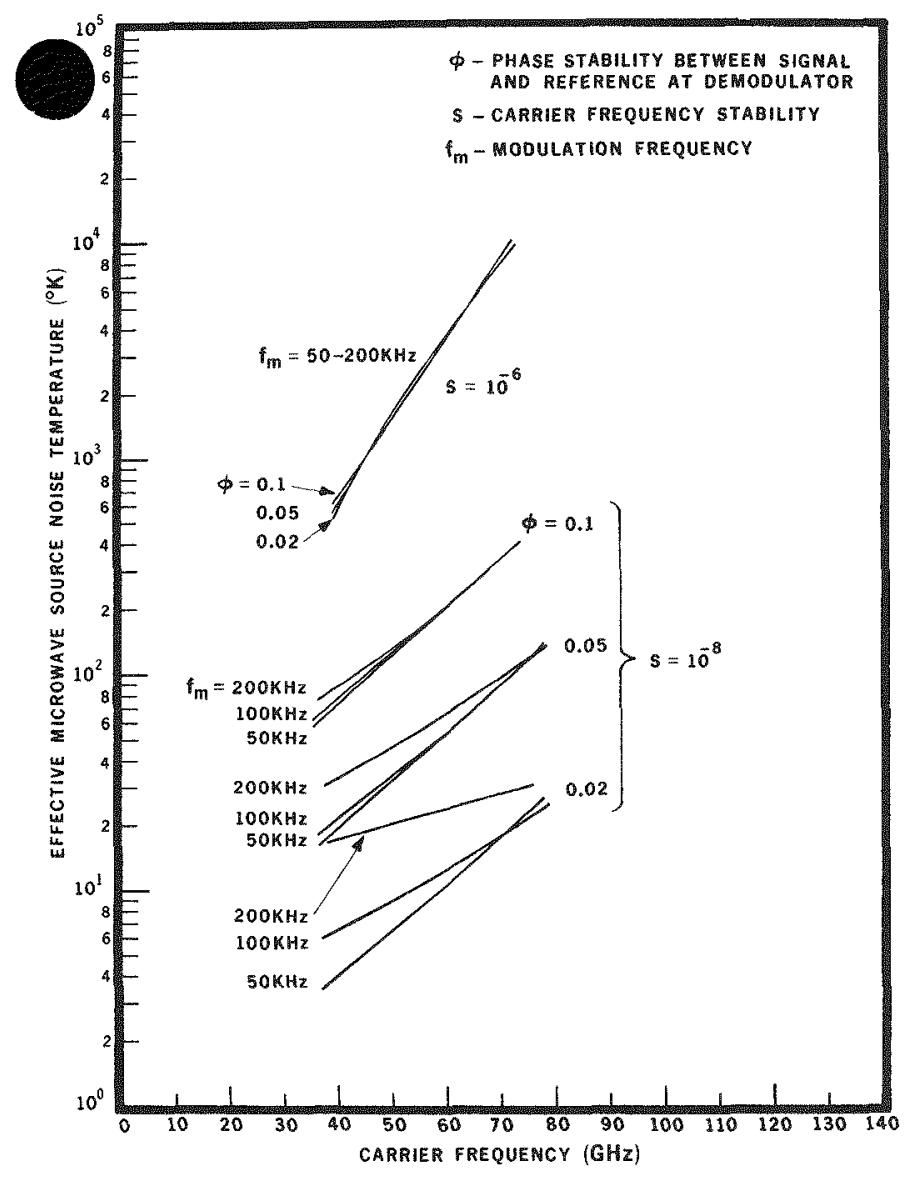

Fig. 2. Effective noise temperature of the microwave source as a function of carrier frequency with the carrier frequency stability $\mathrm{S}$, the phase stability $\phi$ and the modulation frequency $f_{m}$ as parameters, for a carrier power level $\mathrm{p}^{\mathrm{m}}=100 \mathrm{~W}$ and a resonant cavity $\mathrm{Q}-$ factor $=10$.

(XBL 751-87) with $f_{m}, S$, and $\phi$ as parameters, has been calculated and is plotted over a wide range of operating conditions in Fig. 2. The curves were calculated for a carrier power level $P_{0}=100 \mu \mathrm{W}$, and a reflection cavity $Q_{0}$-factor of $10^{4}$, assuming that $\Gamma_{0}^{2}=\left(Q_{0} f_{m} / F_{0}\right)^{2}$.

The curves in Fig. 2 show that the effective noise temperature substantially depends upon the carrier frequency stability $S$ and the phase stability $\phi$. For $S=10^{-8}$, the proper choice of $\phi$ and $f_{m}$ minimizes the effective noise temperature. For $\mathrm{S}=10^{-6}$, the noise temperature depends primarily upon the carrier frequency value.

A comparison of the microwave source noise with the absorption cell noise and the microwave receiver noise shows that the microwave source noise contributes significantly to the total spectrometer noise and ultimately limits the achievable spectrometer sensitivity. It is of particular interest now to further investigate the influence of microwave source noise in a high-sensitivity microwave spectrometer incorporating a phase-sensitive detection system. 6 These studies will give direction for the development of an optimized spectrometer, particularly suitable for measurements of extremely weak absorption lines and studies of the molecular properties of short-lived transient chemical species.

\section{FOO'TNOTES AND REFERENCES}

1. W. F. White, Microwave Spectra of Some Sulfur and Nitrogen Compounds, NASA TN D-7450, May 1974.

2. W. H. Kirchhoff, J. Mol. Spectrosc. 41, 333 (1972).

3. C, H. Townes and A. L. Schawlow, Microwave Spectroscopy McGraw-Hi11, New York, 1955.

4. R. H. Schwendeman, J. Mo1. Spectrosc. 7, 280 (1961).

5. M. W. P. Strandberg, Rev. Sci. Instr. 43, 307 (1972)

6. B. Leskovar, IEEE Transactions in Instrumentation and Measurements, IM-21, No. 1, 15 (1972).

HIGH PURITY GERMANIUM *

William L. Hansen and Eugene E. Ha1ler

\section{INTRODUCTION}

Our work on the development of high purity germanium as a radiation detector material has now progressed enough that some general statements can be made about our present understanding and the areas where further research is needed can be rather sharply defined. The recent application of Fourier Transform IR Spectroscopy has led to the identification of all the important impurities. Several hundred detectors of a11 sizes have been - Je and, in the process, crystal properties inant to device performance have been illuminated. evolution of our present understanding can be divided roughly into three areas: 1) the role of excess vacancies, 2) impurities and impurity distributions and 3) the concern for dislocations and structural defects.

\section{THE ROLE OF EXCESS VACANCIES}

An important acceptor level in high purity gemanium can be produced by an excess of vacancies over the ambient temperature equilibrium value, Crystals grown from a melt incorporate a concentration of vacancies equivalent to their solubility at the melting temperature. As the crystal cools it becomes strongly super-saturated unless some low energy condensation muclei are present. In germanium the vacancy solubility at the melting point is $>10^{15} / \mathrm{cm}^{3}$ and at room temperature it is insignificant.

If no condensation nuclei are present (e.g., dislocations), the strong super-saturation of vacancies causes their precipitation as vacancy clusters or voids. These vacancy clusters, which 
can be revealed by chemical etching (Fig. 1), are always accompanied by acceptor centers with an activation energy of about $80 \mathrm{meV}$ and a concentration of 2 to $41011 / \mathrm{cm}^{3}$ (Fig. 2). Centers with this activation energy are efficient traps at $77 \mathrm{~K}$ and as a result, detectors containing vacancy clusters give completely unacceptable performance.

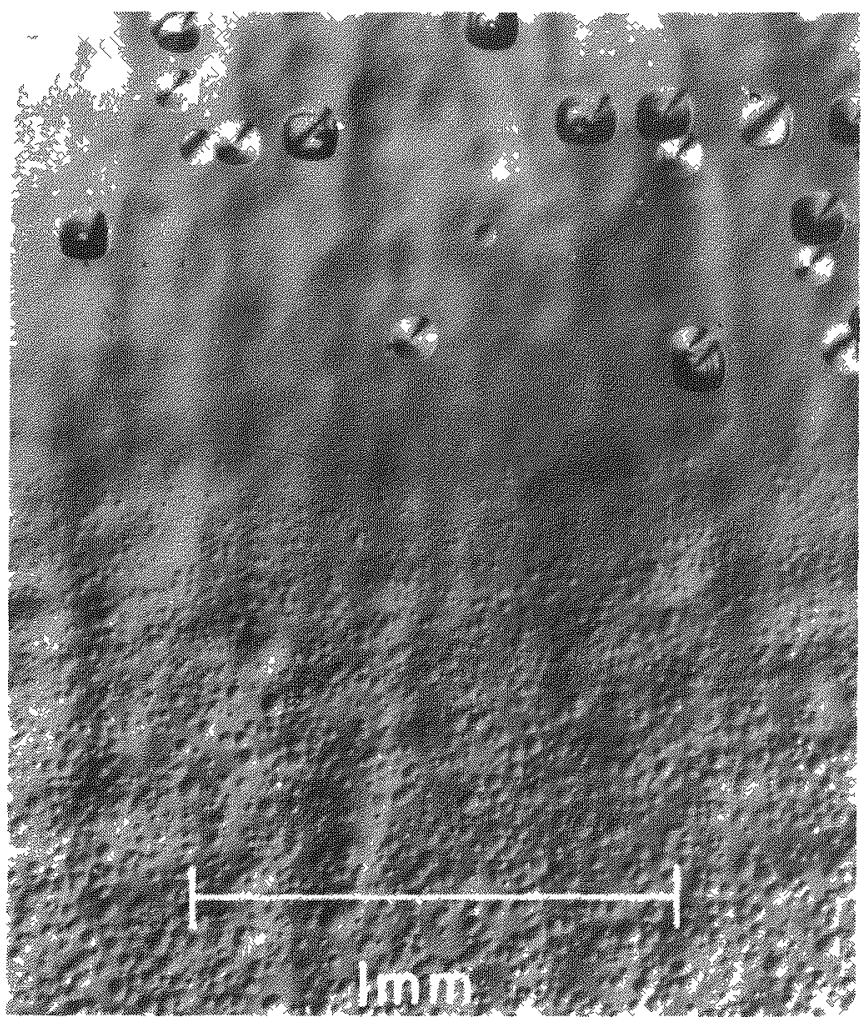

Fig. 1. Photograph of etch pits at the boundary of a dislocated and undislocated region of a crystal slice. In the upper portion are large pits due to disolocations and the lower, small pits due to vacancy clusters. The boundary region is free of pits.

(XBB 7310-6167)

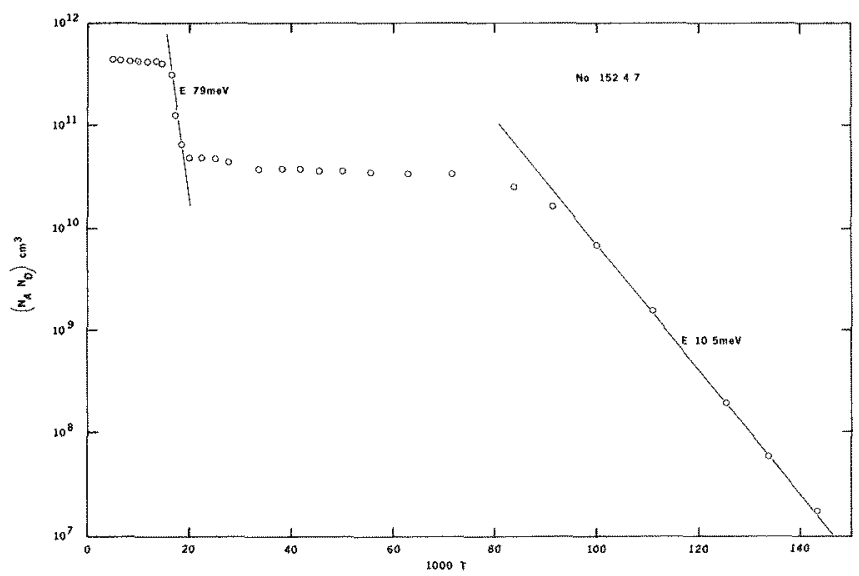

Fig. 2. Acceptor concentration vs. temperature for a dislocation free crystal. The energy leve1 at $79 \mathrm{MeV}$ is associated with vacancy clusters and the level at $10.5 \mathrm{MeV}$ has been determined by FTS as being due to aluminum.

(XBL 7310-1380)

\section{IMPURITIES AND IMPURITY DISTRIBUTIONS}

Most of our crystals have a background dono impurity that segregates toward the tail end so that this end is usually n-type. Many crystals exhibit a uniform net acceptor concentration along most of the length with the tail becoming n-type. Analysis of these crystals by Fourier Transform Spectroscopy (FTS) has shown that the n-type impurity is phosphorus and the p-type is aluninum. A high concentration is sometimes found at the head ends of crystals. FTS shows that this is due to boron which always segregates to this end. Furthermore, these crystals have all been grown from the head ends of zone refined bars. Occasionally crystals are found in which the acceptor concentration increases from the head to the tail. FTS shows that this impurity is gallium or, in a few cases, indium. On the basis of the distribution of the four impurities, we can explain all of our resistivity profiles. Indeed, from an examination of the profile, we can usually infer the nature of the impurities in a given crystal.

\section{THE ROLE OF ALUMINUM}

Having taken into account the effects of excess vacancies, the most common type of impurity distribution that needs explaining is shown by the examples in Fig. 3. As can be seen in this figure, the conductivity of the crystals appears to be dominated by an acceptor with a segregation coefficient very close to 1.0. Low temperature measurements give an activation energy near $10 \mathrm{meV}$ for this acceptor. All of the group III and $V$ impurities have activation energies near $10 \mathrm{meV}$

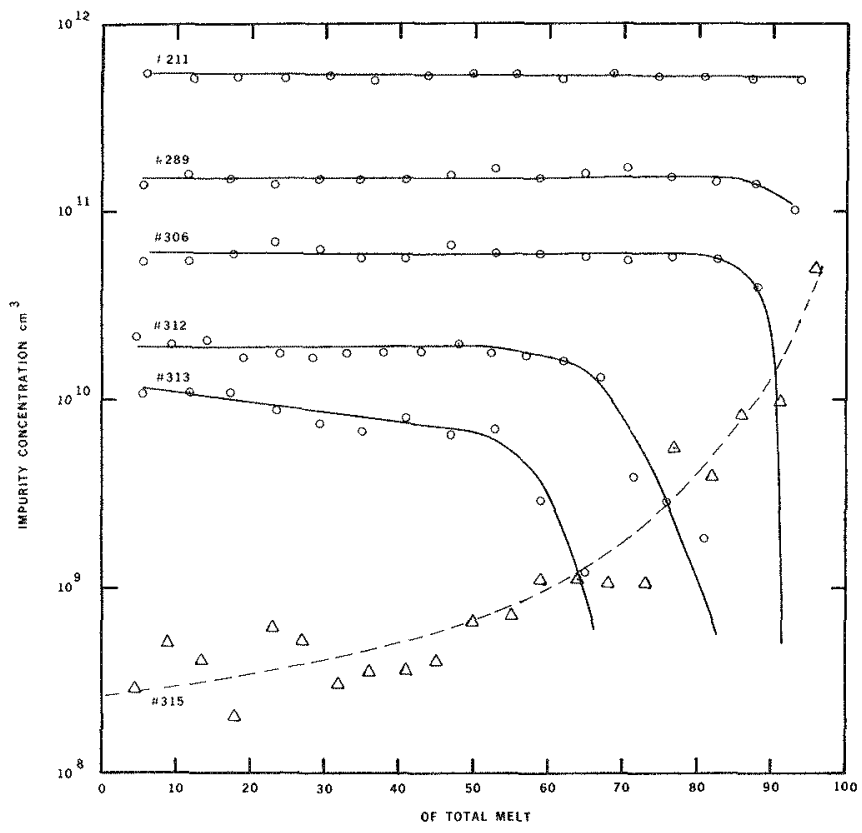

Fig. 3. A selection of crystal profiles which sh the common constant impurity concentratior which have been shown by FTS as due to aluminum. The n-type portions are not shown. The lower dashed profile is of an n-type crystal which apparently contains little aluminum.

(XBL 7310-1341) 
but have segregation coefficients far from 1.0 inder our crystal growing conditions. The segregation coefficients of other common elements are known in gemanium, and all are very far from 1.

Application of FTS to crystals exhibiting the non-segregating impurity proved that this impurity was always aluminum -- even in the purest crystals. Since low temperature conductivity measurements indicated that the total impurity concentration was due to levels near $10 \mathrm{meV}$, and FTS showed only the single dominant spectrum of aluminum, virtually all the electrical activity is accounted for without the need to invoke other sources such as vacancies, dislocations, strain, etc.

\section{CRYSTAL PERFECTION AND GROWING CONDITIONS}

A strong correlation has been observed between poor charge collection in detectors and dislocation density. When the crystal contains $>10^{4} /$ $\mathrm{cm}^{2}$ dislocations, detectable trapping appears that increases with dislocation density. The limits on dislocation density for crystals to make high quality detectors appear to be about 500 to $5000 / \mathrm{cm}^{2}$ and these must be reasonably uniformly distributed. The high or uneven distribution of dislocations is due to poor thermal conditions in the crystal grower. Enough is known historically about how to grow high-quality gemanium crystals except for the fact that we are constrained to grow in hydrogen gas as the only ambient that will produce crystals free of trapping. Hydrogen has a very high thermal conductivity and a low viscosity so it is a source of strong thermal convection and surface cooling of the growing crystal.

\section{DETECTORS}

The process of making detectors from high purity germanium is remarkably free of art and complications when compared with making $1 \mathrm{ithium-}$ drift devices. Stated simply, good germanium makes good detectors - any fussing with the fabrication is usually an attempt to compensate for some defect in the crystal.

If a crystal has sufficient purity to be depleted at a reasonable voltage, we find the device performance to be quite indifferent to typeness or even if of mixed typeness (e.g., an n-type core in a p-type crysta1). Since the field at the metal surface-barrier is lower with $\mathrm{p}$-type crystals these often make devices capable of sustaining higher reverse bias than $n$-type. This may allow using poorer quality p-type than n-type crystals, but good quality crystals do not require high voltage. On the other hand, effectively thinner entry windows in $X$-ray detectors result from using $n$-type materia1.

As larger detectors are made, the crystal ality requirements become more stringent. Since ectral line widths are much more sensitive to differential than absolute charge collection efficiency, the inclusion of regions having a greater range of efficiencies in large devices makes their performance much poorer than small devices made from the same material.
In addition to the problem of material selection, coaxial detector fabrication presents more problems than do planar detectors due to the geometry of the surface barrier. Despite this, our group has made large coaxial detectors. Figure 4 shows a ful1 energy spectrum of 60 Co made with a $43 \mathrm{~cm}^{3}$ coax detector with an external surface barrier and a lithium-diffused core.

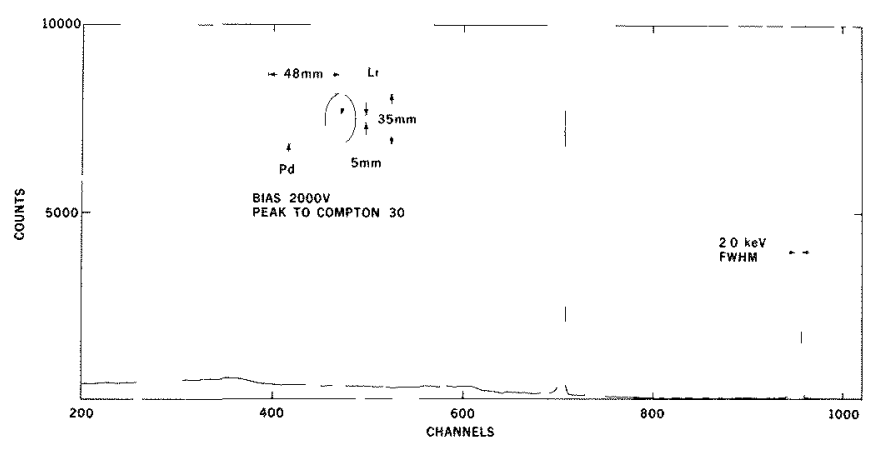

Fig. 4. Portion of a ${ }^{60} \mathrm{Co}$ spectrum taken with a $43 \mathrm{~cm}^{3}$ coaxial high-purity germanium detector. Resolution and peak-to-Compton ratio were determined from expanded plots of this spectrum.

(XBL 7310-1372)

\section{CONCLUSIONS}

The principal source of contamination in much of our high-purity germanium has been shown to be aluminum in the starting material. Fourier Transform Spectroscopy permits analysis of polycrystalline germanium for very low concentrations of p-type impurities, so we are confident that the aluminum impurity can be controlled in the future.

Dislocation densities greater than about $10^{4} / \mathrm{cm}^{2}$ result in detectors with noticeable trapping; consequently crystals used to make high quality detectors should have a dislocation density of 500 to $5000 / \mathrm{cm}^{2}$ with reasonably uniform distribution.

As to detectors, the fabrication process presents no fundamental problems and the particuular methods used are not by any means as critical as the choice of material. Excellent sma11 detectors can be made from most high-purity germanium but routine production of large detectors such as coaxial units of $>50 \mathrm{cc}$ volume awaits sufficient supply of crystals with good structural perfection over large volumes.

While the main problems in producing highpurity germanium for high quality detectors are now clearly delineated, we do not wish to imply that the solution of these problems will be easy. In fact, we anticipate that considerable work will be needed to develop processes capable of producing substantial quantities of large diameter material suitable for large $\left(50 \mathrm{~cm}^{3}\right)$ detectors.

\section{Footnote}

* Condensed from LBL-2435; published in IEEE Trans. Nuc1. Sci. 21, 251 (1974). 
IMPURITIES IN HIGH-PURITY GERMANIUM AS DETERMINED BY FOURTER TRANSFORM SPECTROSCOPY*

Eugene E. Haller and William L. Hansen

\section{INTRODUCTION}

During the last few years a great deal of work has accumulated toward characterizing highpurity germanium in respect to the fabrication of radiation detectors. Many techniques were adapted and modified to complete this rather difficult task. Hall effect, conductivity measurements, and metal-point probing on bulk material as well as capacity-voltage dependences and alphaparticle probing of detectors and detector arrays helped to find the axial and radial distribution of donors and acceptors.1,2 However, very little work was done on analysis to identify the impurities. The main reason was the lack of a method useful at net-concentrations below $10^{10} / \mathrm{cm}^{3}$. Since the remaining impurities are usually accidental (i.e., not added intentionally) it is of crucial interest to find their nature and hence, perhaps, their source. 3

Photothermal ionization of neutral acceptors/donors as discovered by Russian scientists, 4-6 combined with Infrared Fourier Transform Spectroscopy (FTS) takes advantage of the properties of high-purity germanium in a unique way. This was shown by S. D. Seccombe and D. M. Korn 7 with $n^{-}$ type material produced by R. N. Hall at General Electric Research Center, Schenectady, New York. We have now applied the same method to p-type material down to net-acceptor concentrations of below $10^{9} / \mathrm{cm}^{3}$.

It was shown theoretica11y and experimentally that impurities in semiconductors have more than one bound state for their holes/electrons and produce "hydrogen-like" excitation spectra. Figure 1 presents the experimentally determined

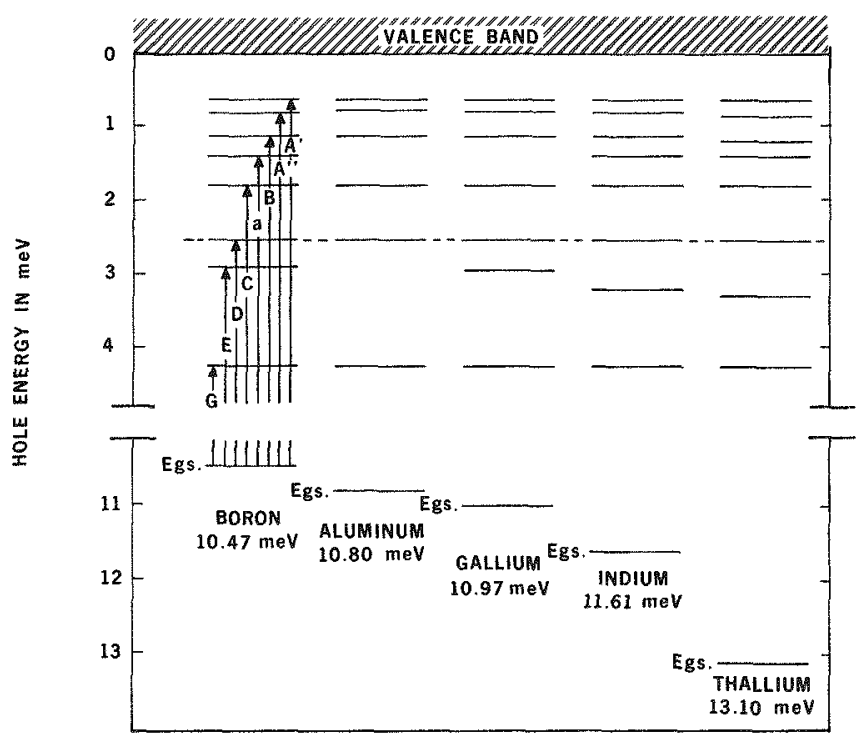

Fig. 1. The bound states of the group III impurities in germanium. Energies of the states and the labeling of the transitions are the same as in Ref. 8. level schemes for neutral acceptors in germanium. ${ }^{8}$ Similar sets of excited states are produced by group $V$ impurities in $n$-type germanium 9 as well as in any other electrically active centers.

\section{THE MEASUREMENT OF IMPURITIES}

Use of an interferometer instead of a spectrograph makes possible measurements of the required sensitivity in a short time. Interferograms are converted into frequency spectra by Fourier transformation. The signal processing and data handling systems are shown schematically in Fig. 2B. Chopped infrared radiation enters the germanium sample causing photothermaI excitation which changes the free carrier concentration. The change in voltage across the symmetrically biased sample is amplified with high common-mode rejection and fed into a conventional phase-lock amplifier (PAR mode1 H-8). This unit filters, amplifies and synchronously rectifies the signal, as well as controlling the chopper motor. A voltage to frequency converter produces a frequency proportional to the signal.

The lower half of Fig. 2A shows a typical interferogram produced by a cube-shaped $\left(0.57 \mathrm{~cm}^{3}\right)$ sample \#313-0,2. The net acceptor concentration was about $10^{10} \mathrm{~cm} 3$. Because we do not understand details of the signal formation process in the detector we can only state some experimental observations. In general the signal rises with increasing bias across the sample. We attribute this to multiple refilling and excitation of the acceptor states during the time light enters the sample. However, at a certain bias, breakdown occurs and the signal becomes very noisy. The critical value of the bias depends on the net-carrier concentration, sample surface preparation and on the temperature; a few hundred millivolts is a typical value for $8-\mathrm{mm}$ thick samples. The signal amplitude does not depend on chopping frequency from a few cycles per second up to $100 \mathrm{~Hz}$, the limit for the chopper motor. This means that carrier relaxation times in the sample are shorter than $\sim 1 \mathrm{~ms}$ ( $\omega \tau \leq 1$ ).

Aluminum plays the most important role as an impurity in our high-purity gemanium. 3 It was found to be the only important impurity in many crystals with net-acceptor concentrations ranging from $>10^{11} / \mathrm{cm}^{3}$ down to a few times $10^{9} / \mathrm{cm}^{3}$. A spectrum of the sample \#313-0.2 containing mostly aluminum is displayed in Fig. 3. The lines of $A 1(A-G), B(A-G)$ and $G a(A-G)$ correspond to those published by R. L. Jones and $P$. Fisher 8 within their experimental exrors $(\sim 0.2 \%)$. The continuum of Al starting at about $10.8 \mathrm{meV}$ is produced by holes which are excited directly from the ground state into the valence band.

The line-widths at half-maximum are limited by the instrument since the total mirror-advance is $2 \mathrm{~cm}$. As can be seen in Fig. 2, the amplitude of the interferogram for this same sample does not decay to zero even at $x=2 \mathrm{~cm}$. This means 


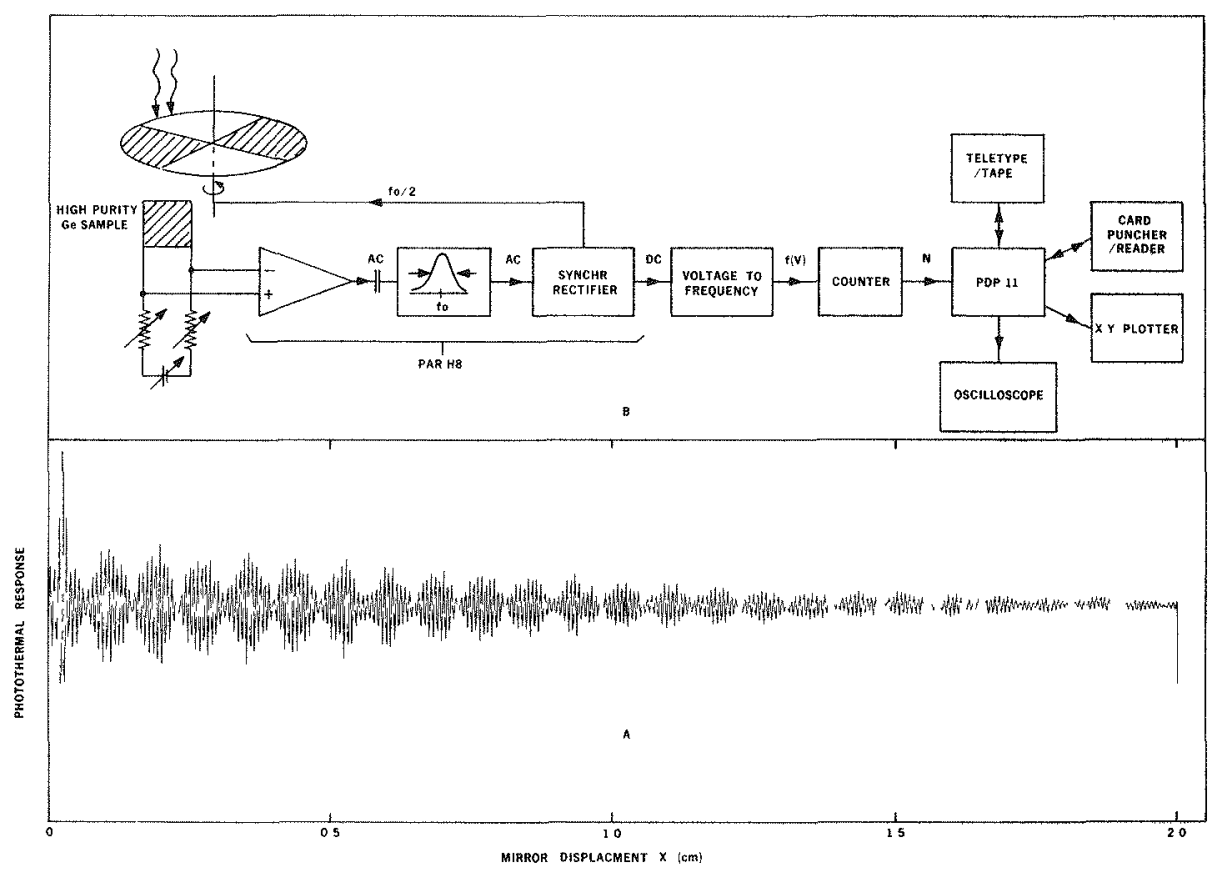

Fig. 2. Interferogram obtained from sample \#313-.2. Sample volume $=.57 \mathrm{~cm}^{3}$; $\mathrm{N}_{\mathrm{A}}-\mathrm{N}_{\mathrm{D}}=10^{10} \mathrm{\textrm {cm } ^ { 3 }} ; \mathrm{T}=8.0^{\circ} \mathrm{K} ; 1000$ sampling points were recorded in approximately 30 minutes. The "beat" character of the interferogram is produced by the strong $\mathrm{Al}(\mathrm{C})$ and $\mathrm{Al}(\mathrm{D})$ lines.

Signal processing and data handling system used in connection with the interferometer. The germanium sample simultaneously acts as absorber and detector of IR-radiation.

(XBL 7311-1401)

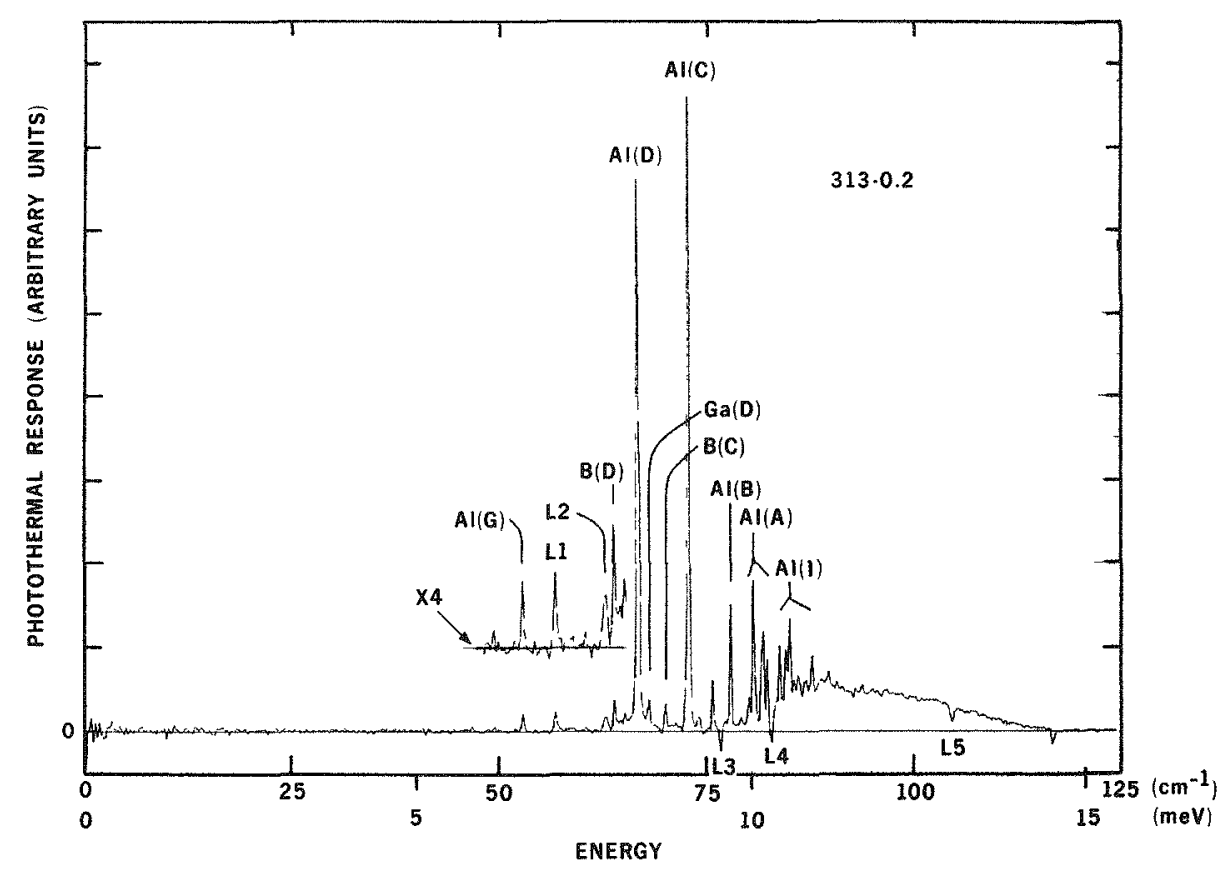

Fig. 3. Spectrum of sample \#313-.2. The labeling of the lines is the same as in Ref. 8. Sample volume $=.57 \mathrm{~cm}^{3} ; \mathrm{N}_{\mathrm{A}}-\mathrm{N}_{\mathrm{D}}=10^{10} / \mathrm{cm}^{3} ; \mathrm{T}=8.0^{\circ} \mathrm{K} ; 1000$ sampling points were recorded in approximately 30 minutes. A region below the A1(D) line is magnified by a factor of four with the baseline shifted. The aluminum concentration exceeds all other impurities (B, Ga) by a factor of more than 20 . The explanation for the lines $L_{1}$ through $\mathrm{L}_{5}$ is given in the text. 
that the natural line-width of some lines is smaller than $0.25 / \mathrm{cm}$.

The ratio of corresponding lines of different impurities approximately represents the ratio of their concentrations al though a correction must be applied because of the variation of intensity of the exciting light with the wave number. This dependence is weak in the spectral region of the strong $\mathrm{D}$ and $\mathrm{C}$ 1ines.

Using this simple fact together with Halleffect measurements of the samples we can observe the segregation of all impurities separately along the length of the crystal. The segregation coefficients for boron and gallium are very reasonable for the particular crystal growing parameters. The anomalous behavior of aluminum once again is evident.

The quality of spectra obtained with the full resolving power of the instmment is illustrated in Fig. 4 where four impurities are present (sample \#291-14.0). The $A 1(C)$, In(D) and the $\mathrm{Ga}(\mathrm{C})$ lines are clearly separated. Their relative spacings are below $0.1 \mathrm{meV}$. In addition to the impurities $\mathrm{Al}$, In and $\mathrm{Ga}$, a new unknown shallow acceptor $X$ appears. The relative spacings between the (D), (C) and (B) 1ines of $X$ are in agreement with the other shallow acceptor lines. We determined the ground state energy $\mathrm{E}_{\mathrm{g} . \mathrm{s}}$ as $0.18 \mathrm{meV}$ above the ground state of boron, i.e., (10.47 +0.18 ) meV. From measurements of several samples along crystal \#291 we conclude that the segregation coefficient of $X$ is somewhat smaller than that of Gallium and probably lies around 0.05. An n-type core makes a more precise estimate based on this particular crystal impossible.
A second unknown shallow acceptor (impurity $Y$ ) was first observed in a polycrystalline sample from a very high-purity zone refined gemanium bar and later in two crystals pulled from a quartz, and in one crystal grown from a carbon crucible. In the latter crystal the impurity $Y$ was present at a rather high concentration of $\sim 10^{10} \mathrm{~cm}^{-3}$. From Hall effect and IR-measurements we found the segregation coefficient of $Y$ to be $k Y=0.9 \pm 0.1$. This means that $Y$ is nearly non-segregating in contrast to the dominant impurity aluminum, which segregates normally in crystals grown from carbon crucibles. This behavior could be explained by an impurity which diffuses out of the carbon crucible into the Ge-melt and quickly reaches its rather low maximum solubility.

The spectrum of an n-type sample \#296-15.0 is shown in Fig. 5. InGa contacts rubbed onto opposite surfaces of the polish-etched cubic sample were used. All the lines can be explained on the basis of phosphorus as an impurity. 9 Noise in the spectrum is due to current injection noise. Almost no improvement in current injection resulted from using lightly 1 ithium-doped contacts, but this introduced the Li-lines. Only in Hoboken LMC material which was $n$-type did we find lines corresponding to antimony and to 1 ithium without exposing the material to any heat treatment.

We were pleasantly surprised to discover that the method works also for polycrystalline material. Line splitting is observed in some samples, most probably due to stress, but it is not severe enough to make the analysis of the impurities questionable.

In order to test the method for "deep"

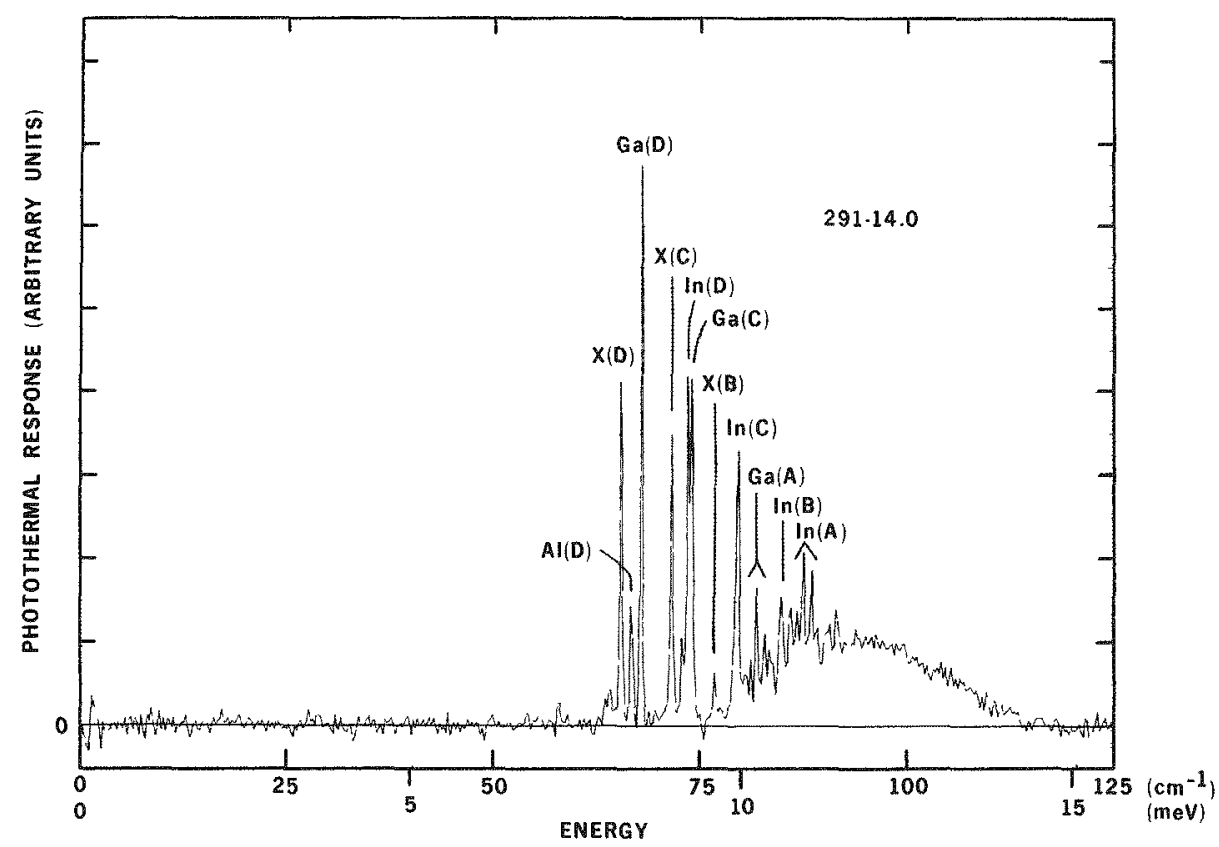

Fig. 4. Spectrum of sample \#291-14.0. Sample volume $=.47 \mathrm{~cm}^{3} ; \mathrm{N}_{\mathrm{A}}-\mathrm{N}_{\mathrm{D}}=10^{11} / \mathrm{cm}^{3}$; $\mathrm{T}=8.0^{\circ} \mathrm{K} ; 1024$ sampling points were recorded in 30 minutes. Four impurities are present: $X$ (unknown), $\mathrm{Al}, \mathrm{Ga}$ and $\mathrm{In}$. The resolution allows the separation of the $\mathrm{Ga}(\mathrm{C})$ and $\mathrm{In}$ (D) Iines.

(XBL 7310-1396) 


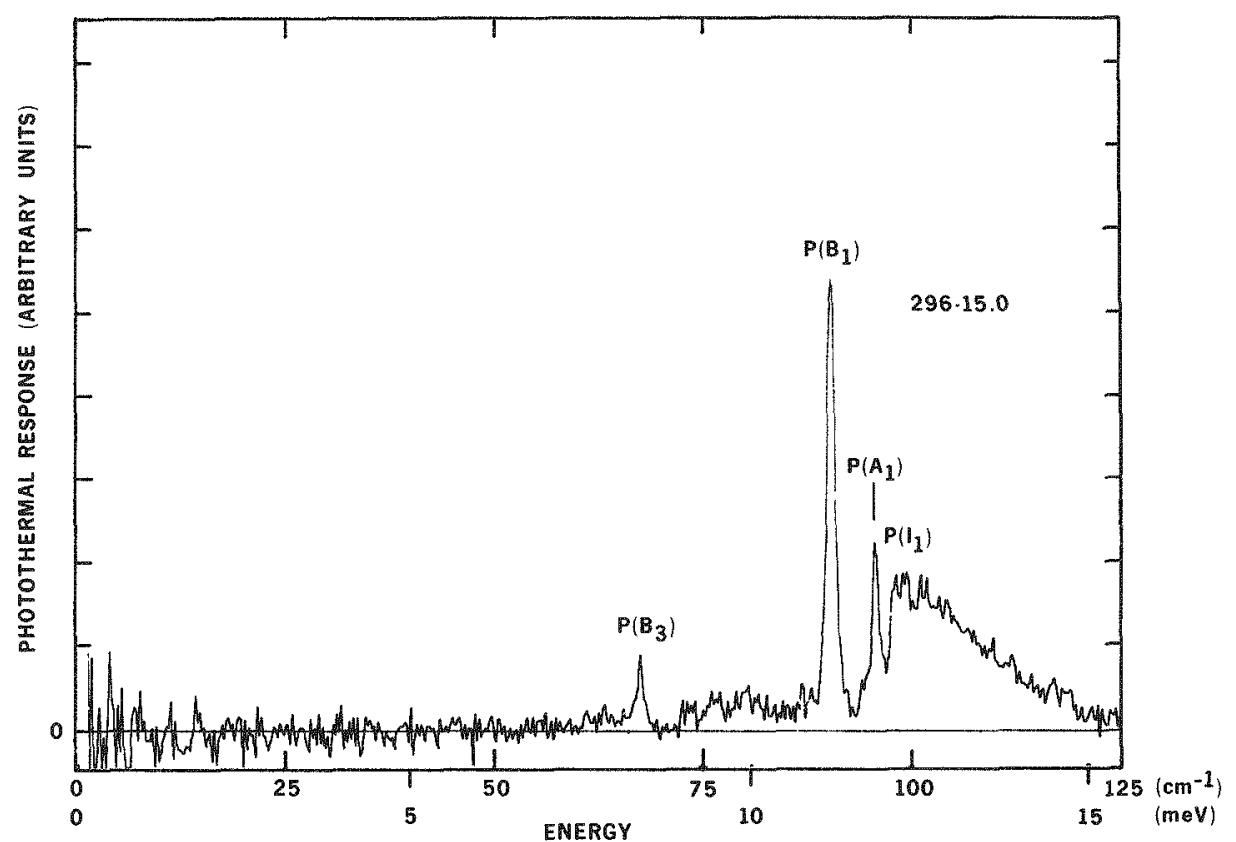

Fig. 5. Spectrum of the $n$-type sample \#296-15.0. Sample volume $=.42 \mathrm{~cm}^{3} ; \mathrm{N}_{D^{-}} \mathrm{N}_{\mathrm{A}}$ $=610^{11} / \mathrm{cm}^{3} ; \mathrm{T}=6.5^{\circ} \mathrm{K} ; 700$ sampling points were recorded in approximately 30 minutes. Phosphorus is the only detectable donor imputity in our $\mathrm{n}$-type material.

(XBL 7310-1395)

acceptors we measured the photothermal response of high-purity, dislocation-free germanium up to a wave number of $500 / \mathrm{cm} \quad(=61.8 \mathrm{meV})$. This is the upper limit for the instrument available to us. We were able to detect neutral beryllium 10 in dislocation-free material.

\section{CONCLUSIONS}

We have shown experimentally that the combination of Fourier Transform Spectroscopy (FTS) together with photothermal ionization of neutral impurities makes possible the analysis of acceptors and donors in high-purity germanium down to netconcentrations of $\sim 10^{9} / \mathrm{cm}^{3}$ with a signal to noise ratio $>100$. With the instrument available to us we did not reach a resolution comparable to the natural line-width of shallow acceptors. We believe, however, that a better resolution will not improve the analytical power of the method. Together with Hall-effect, which is the best tool for the measurement of the net-concentrations, the segregation of each impurity separately along the axis of a crystal can be determined. That the method works especially well for shallow acceptors and donors is partially due to the fact that both the current through the sample and the temperatures involved are 10w. The only deep acceptor detected was neutral beryliium $\left(E_{g} . s=24.3 \mathrm{meV}\right)$. Whether the method will be applicable to deeper levels remains an unanswered question until instruments with a higher maximum wave number $\nu_{\max }$ are used.

From measurements of over 50 samples we found

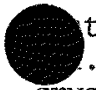
aluminum is the main impurity in p-type materThe presence of boron at the seed-end of some crystals can be predicted from the shape of the concentration profile obtained from conductivity measurements.

With the increase of the sample volume we would be able to analyze any impurities producing shallow levels down to the $10^{7} / \mathrm{cm}^{3}$ range.

\section{ACKNOWLEDGEMENTS}

P. Richards has kindly allowed us to share in the use of his busy Fourier Transform Spectrometer, without which we could not have performed the FTS work. He has also been generous in giving his time for discussing many aspects of the theory and practice of FTS.

\section{Footnotes and References}

* Condensed from LBL-2436 and LBL-2487; pub1ished in IEEE Trans. Nuc1. Sci. 21, 279 (1974) and So1. State Comm. 15, 687 (1974).

1. Hal1, R. N., Baertsch, R. D., Soltys, T. J. and Petrucco, L. J., "High-Purity Germanium for Gamma Detectors", General Electric Co. Annual Reports Numbers 1 through 5.

2. Haller, E. E., Hansen, W. L. and Goulding, F. S., IEEE Trans. Nuc1. Sci., 20, No. 1, 481 (1973)

3. Hansen, W. L. and Haller, E. E., IEEE Trans. Nuc1. Sci., 21, No. 1, 251, (1974).

4. Lifschitz, T. M. and Ya.Nad', Sov. Phys. Doklady, 10, No. 6, 532 (1965).

5. Sidorov, $\overline{V_{0}}$ I. and Lifschitz, T. M., Sov. Phys. Solid State, 8, No. 8, 2000 (1967).

6. Kogan, Sh. M. and Sedunov, Sov. Phys. Solid State, 8, No. 8, 1898 (1967).

7. Seccombe, S. D. and Kom, D. M., Solid State Conm. . 11, 1539 (1972).

8. Jones, R. L. and Fisher, P., J. Phys. Chem. Solids, 26, 1125 (1965).

9. Reuszer, J. H. and Fisher, P., Phys. Rev., 135, A1125 (1964).

10. Shenker, H., Swiggard, E.M. and Moore, W.J., Trans. Meta1, Soc. of AIME, 239, 347 (1967). 
APPLICATION OF A LOW ENERGY X-RAY SPECTROMETER TO ANALYSES OF SUSPENDED AIR PARTICULATE MATTER*

R. D. Giauque, R. B. Garrett, L. Y. Goda, J. M. Jaklevic, adn D. F. Malone

\section{INTRODUCTION}

The analytical technique of $\mathrm{X}$-ray induced $X$-ray Fluorescence Analysis (XRFA) lends itself to the simultaneous determination of a broad range of elements in environmental specimens. Both energy dispersive and wavelength dispersive spectrometers are presently employed at various laboratories for the analyses of air particulate specimens collected on thin substrata.

XRFA methods are ideal for the analysis of many of the heavier elements in air particulate samples. Characteristic $X$-rays from these elements are not absorbed within the sample (the sample is said to be "infinitely thin") and the elemental concentrations are directly proportional to intensities of the characteristic X-ray lines. For the lighter elements $(Z<20)$, the characteristic $X$-rays undergo significant absorption within the sample. Absorption correction methods reported have been based on assumptions of the particle compositions and of the particle size distributions for nonsize segregated air particulate specimens. 1,2 Models for deriving corrections for absorption effects due to particle penetration in the filter media have also been suggested. 2,3

We have developed a technique for performing energy dispersive analysis of light elements in air particulate samples. An X-ray spectrometer especially designed for the detection of low-energy $\mathrm{X}$-rays has been constructed and used to measure the concentration of both high and low (down to $\mathrm{Mg}$ ) atomic number elements. A semi-empirical technique to compensate for particle size effects has been applied to the analysis. This technique makes use of multiple energies of X-ray excitation which act as variable depth probes when determining the concentration of the light elements. Our results compare well with those obtained by Neutron Activation Analysis (NAA).

\section{INSTRUMENTATION}

A number of approaches have been used in previous $X$-ray systems to generate variable energies of excitation radiation, including multiple $X$-ray anodes 4 and secondary fiuorescence targets. 5,6 As an alternative approach, we have designed a spectrometer which permits the use of both direct and indirect excitation radiation by using either transmission filters or secondary targets respectively as shown in Figs. 1 and ?. This design allows the excitation radiation to pass through the vacuum cryostat, giving very large solid angles and minimu $X$-ray attenuation. The air path length between the specimen and the detector window $(0.002 \mathrm{~cm} \mathrm{Be})$ is only $1.5 \mathrm{~mm}$. This enhances sensitivity for low-Z elements. Also, with this system, sma11 tota1 specimen areas, $1 \mathrm{~cm}^{2}$, are required.

In the direct excitation mode, high-excitation efficiencies are achieved for $10 \mathrm{w}-Z$ elements using AgL X-rays, and for high-Z elements using AgK X-rays. Transmission Ag filters are inserted to modify the $X$-ray tube output. Typical count rates are $5,000-10,000$ counts/sec using a 10 power X-ray tube ( 40 watts). The detector, which has an effective area of $40 \mathrm{~mm}^{2}$, has been collimated to an area of approximately $30 \mathrm{~mm}^{2}$. This modification enhances sensitivities attainable when AgK $X$-rays are used for analyses. 6,7 It minimizes the fraction of the high-energy radiation detected near the periphery of the sensitive region of the detector, which otherwise would substantially increase the background in the region of interest.

\section{PARTICLE SIZE EFFECTS}

When analyzing for elements such as Al, Si, $S, C 1, K$, and $\mathrm{Ca}$, corrections must often be made

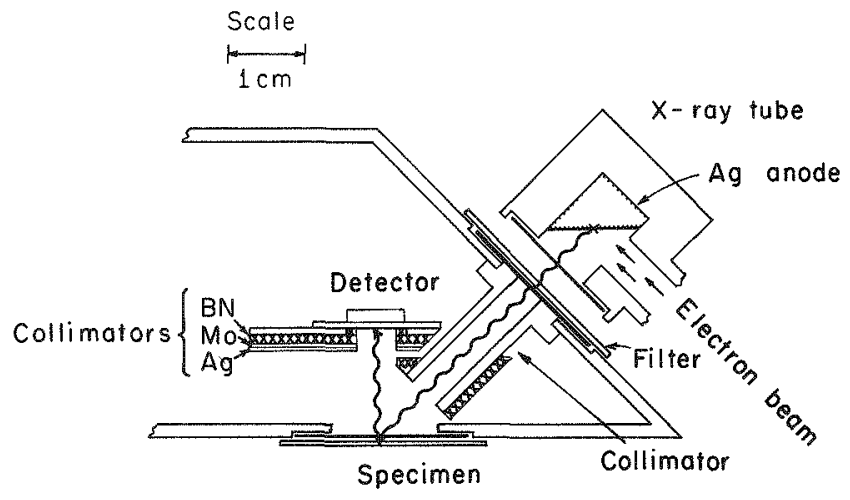

Fig. 1. X-ray spectrometer in direct excitation mode.

(XBL 757-3419)

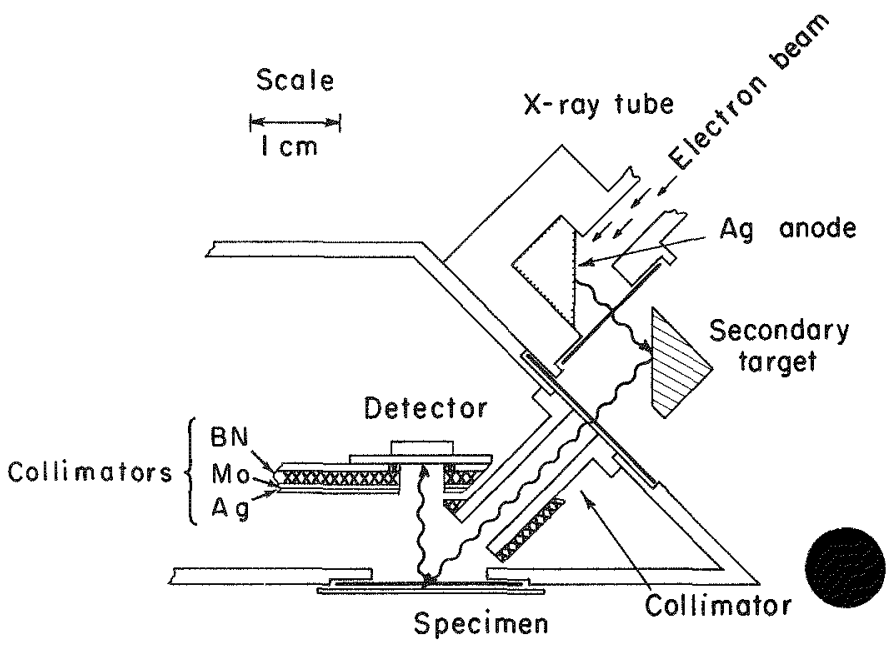

Fig. 2. Spectrometer in secondary excitation mode.

(XBL 757-3420) 
for particle size effects (mainly absorption efcts). The characteristic $X$-rays from these eleents are low in energy, $<4 \mathrm{keV}$, and the "infinitely thin" specimen criteria is usually not valid. In the method we use to make these corrections, relatively low-energy excitation radiations are generated separately from secondary targets in our spectrometer. These radiations act as variable depth probes as described below.

The procedure we use is based on the analysis of the absorption effects in a single particle. The concentration of an element uncorrected for absorption effects, $\hat{c}\left(\mu \mathrm{g} / \mathrm{cm}^{2}\right)$ detemined in a particle of average mass thickness $m$, can be approxmated as:

$$
\hat{c}=c\left\{1-\exp \left[-\left(\mu_{e}+\mu_{f}\right) \bar{m}\right]\right\} /\left(\mu_{e}+\mu_{f}\right)^{\bar{m}}
$$

where $c$ is the true concentration of the element, and $\mu$ and $\mu_{f}$ are the total mass absorption coefficients of the particle for the excitation and fluorescent radiations respectively.

From Eq. 1, if two different energies of excitation radiation are applied separately for analyses, the ratio of the two observed (uncorrected) concentrations is

$$
\begin{aligned}
& \hat{c}_{2} / \hat{c}_{1}=\left\{1-\exp \left[-r\left(\mu_{e_{1}}+\mu_{f}\right) \bar{m}\right]\right\} / r \\
& \quad \times\left\{1-\exp \left[-\left(\mu_{e_{1}}+\mu_{f}\right) \bar{m}\right]\right\}
\end{aligned}
$$

where

$$
r=\left(\mu_{e_{2}}+\mu_{f}\right) /\left(\mu_{e_{1}}+\mu_{f}\right)
$$

and the subscripts 1 and 2 denote the two different excitation energies. If the value of $r$ is known, the quantity ( $\left.\mu_{1}+\mu_{f}\right) \vec{m}$ may be ascertained once the quantity $\hat{\mathrm{c}}_{2} / \hat{\mathrm{c}}_{1}$ is determined. This, in turn, is sufficient information to make an absorption correction as in Eq. (1).

Large particles in atmospheric aerosols originate principally from mechanical processes (e.g. wind-blown soil dust) and usually consist of such minerals as kaolinite, anorthite, microline, and albite. 8 For specific excitation radiations such as ZrL, AgL, and NiK X-rays, the total mass absorption coefficients of these minerals are similar for $\mathrm{Si}$ and $\mathrm{A} 1$. Table 1 shows the ratio of the total mass absorption coefficients [ $r$ in $\mathrm{Eq} \cdot(2)]$ for given pairs of these radiations. For these minerals, the value of $r$ is relatively constant.

Figure 3 shows curves for $r$ values which would be used for $\mathrm{Si}$ or $\mathrm{Al}$ mass concentration determinations in a typical dust particle using $\mathrm{ZrL}$ AgL excitation radiations. These curves plot ratio of observed concentrations versus the correction term to be applied to compensate for absorption effects when analyzing with AgL X-rays. This procedure cannot be used if the particle is "infinitely thick" (i.e., the absorption correction term is $>4$ ).
Table 1. Total Mass Absorption Coefficient Ratios

\begin{tabular}{lcccc}
\hline Excitation Radiations & \multicolumn{2}{c}{ ZrL/AgL } & \multicolumn{2}{c}{ NiK/AgL } \\
\hline Element of Interest & $\mathrm{A} 1$ & $\mathrm{Si}$ & $\mathrm{A} 1$ & $\mathrm{Si}$ \\
\hline Mineral & & & & \\
Kaolinite & 1.51 & 1.49 & 0.77 & 0.78 \\
$\mathrm{Al}_{2}\left(\mathrm{Si}_{2} \mathrm{O}_{3}\right)(\mathrm{OH})_{4}$ & & & & \\
Anorthite $_{\mathrm{CaA1}} \mathrm{Si}_{2} \mathrm{O}_{8}$ & 1.53 & 1.49 & 0.77 & 0.79 \\
Microline $_{\text {KA1Si }} \mathrm{O}_{8}$ & 1.56 & 1.60 & 0.77 & 0.74 \\
Albite & & & & \\
NaAlSi $_{3} \mathrm{O}_{8}$ & 1.53 & 1.58 & 0.75 & 0.72 \\
\hline
\end{tabular}

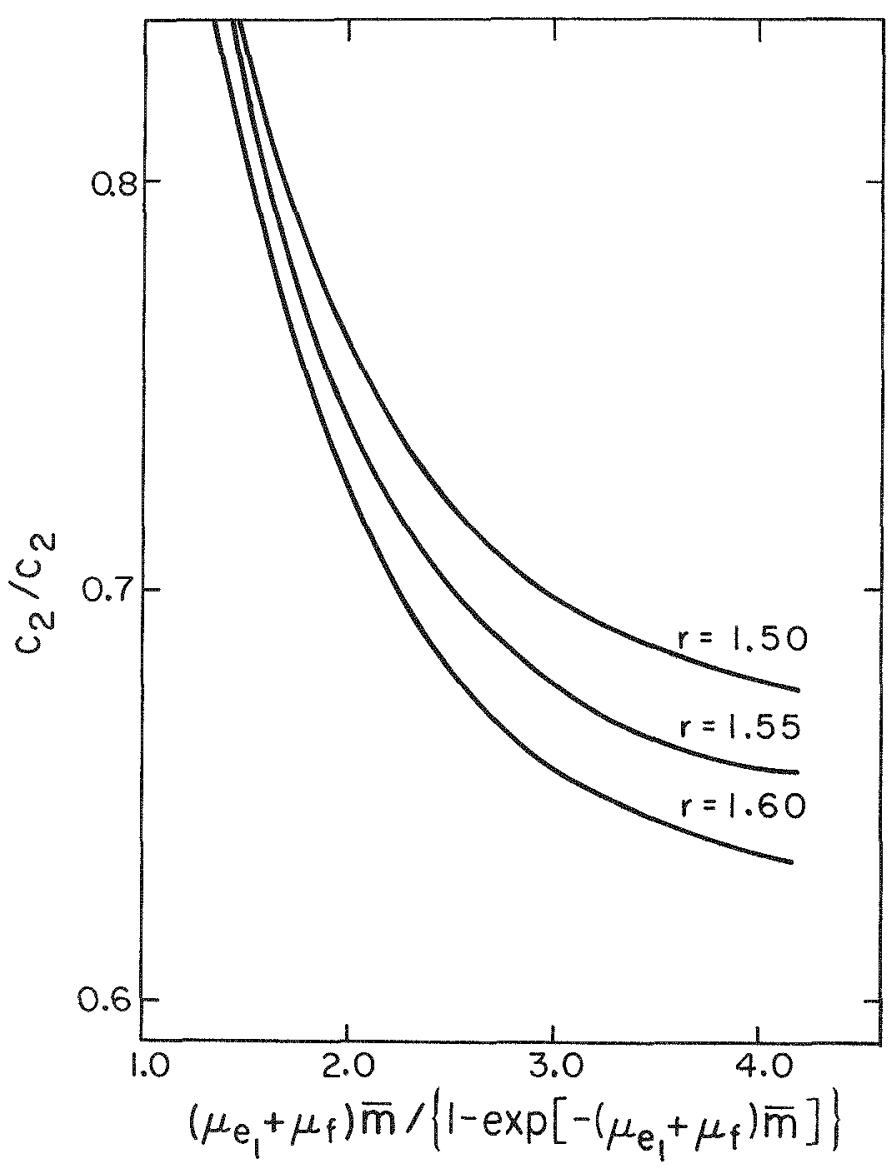

Fig. 3. Absorption correction curves for $\mathrm{Si}$ and $\mathrm{AI}$ mass concentration determination.

(XBL $747-3756 \mathrm{~B})$

In practice, we do not perform analyses on single particles but rather, apply the procedure to a collection of particles; that is, we treat the collection as if it were a single particle of average mass thickness, $\bar{m}$. In this case, the precise physical meaning of the term $\left(\mu_{e}+\mu_{f}\right) \vec{m}$ is somewhat obscure. However, our experience indicates 
that the procedure works rather well because the bulk of the mass concentration is usually contained in a relatively limited size range of particles.

For our determinations with $\mathrm{ZrL}$ and AgL Xrays, we initially select an $r$ value of 1.55 . We make an additional analysis, using $\mathrm{NiK} X$-rays for excitation to verify that we have chosen the correct value of $r$. If many particles of "infinite thickness" are present, verification cannot be obtained. In such a case, a minimum mass concentration is reported.

The advantage of the semi-empirical method for particle size corrections derive from its relative lack of assumptions regarding the size distribution of the collected particles. Size distribution models have limited applicability in general usage, since meteorological conditions and sampling techniques can greatly change the results.

\section{SOME RESULTS}

Table 2 lists a comparison of results acquired by $\mathrm{X}$-ray fluorescence and neutron activation analyses on two-hour aerosol specimens collected during the California Aerosol Characterization Experiment in 1973. The neutron activation analyses were performed by R. Ragaini of the Lawrence Livermore Laboratory. Relatively good agreements were obtained by the two techniques for $\mathrm{A}, \mathrm{Ca}$, and $\mathrm{Br}$. The large uncertainty in the XRF Al value for specimen No. 2 reflects the uncertainty in the ascertained absorption correction factor which was

Table 2. Analysis of Aerosol Specimens

\begin{tabular}{lllll}
\hline No. & Method & $\begin{array}{c}\mathrm{AI} \\
\left(\mu \mathrm{g} / \mathrm{cm}^{2}\right.\end{array}$ & $\begin{array}{c}\mathrm{Ca} \\
\left(\mu \mathrm{g} / \mathrm{cm}^{2}\right)\end{array}$ & $\begin{array}{c}\mathrm{Br} \\
\left(\mu \mathrm{g} / \mathrm{cm}^{2}\right)\end{array}$ \\
\hline \multirow{2}{*}{1} & XRF & $2.4 \pm 0.5$ & $2.3 \pm 0.2$ & $0.21 \pm 0.01$ \\
& NAA & $2.3 \pm 0.1$ & $2.1 \pm 0.4$ & $0.19 \pm 0.01$ \\
3 & XRF & $9.0 \pm 2.7$ & $6.2 \pm 0.3$ & $0.21 \pm 0.01$ \\
3 & NAA & $5.7 \pm 0.1$ & $5.3 \pm 1.9$ & $0.18 \pm 0.01$ \\
& XRF & $0.7 \pm 0.2$ & $0.5 \pm 0.1$ & $0.15 \pm 0.01$ \\
4 & NAA & $1.1 \pm 0.1$ & $0.8 \pm 0.3$ & $0.11 \pm 0.01$ \\
& XRF & $0.3 \pm 0.2$ & $0.3 \pm 0.1$ & $0.15 \pm 0.01$ \\
& NAA & $0.4 \pm 0.1$ & $0.4 \pm 0.2$ & $0.12 \pm 0.01$
\end{tabular}

2.9. As shown in Fig. 3, an uncertainty of three percent in the chosen $r$ value could yield a 15 to $30 \%$ exror in the result for such a large ascertail absorption correction factor.

We have applied our particle size correction technique to the analysis of specimens collected from a number of regions and presumably representing a considerable variation in particle composition. The results obtained have been in good agreement with NAA.

\section{FOOTNOTES AND REFERENCES}

* Research supported by the California Air Resources Board and the USERDA.

1. J.R. Rhodes and C. B. Hunter, Particle Size Effects in X-ray Emission Analysis: Simplified Formulas for Certain Practical Cases, $\mathrm{X}$-ray Spectrom. 1, 113-117 (1972) .

2. T. G. Dzubay and R. O. Nelson, Self Absorption Corrections for $X$-ray Fluorescence Analys is of Aerosols, in Advances in X-ray Analysis, edited by W. L. PickTes (PIenum, New York, 1975), Vo1. 18, pp. 619-631.

3. N. A. Bonner, F. Bazan, and D. C. Camp, Elemental Analysis of Air Filter Samples Using $\mathrm{X}$-ray Fluorescence, Lawrence Livermore Lb. Rprt, UCRL-51388, Livermore, CA (1973) .

4. G. R. Dyer, D. A. Gedcke, and T. R. Harris, Fluorescence Analysis Using an $\mathrm{Si}(\mathrm{Li}) \mathrm{X}$-ray Energy Analys is System with Low-Power X-ray Tubes and Radioisotopes, in Advances in $X$-ray Analysis, edited by K.F.J. Heinrich (Plenum, New York, 1972), Vol. 15, pp. 228-239.

5. J. M. Jaklevic, F. S. Goulding, B. V. Jarrett, and J. D. Meng, Application of X-ray Fluorescence Techniques to Measure Elemental Composition of Particles in the Atmosphere, in Analytical Methods Applied to Air Pollution Measurements, edited by R. K. Stevens and W. F. Herget (Ann Arbor Science Publishers, Inc., 1974), pp. 123146.

6. D. E. Portor and R. Woldseth, X-ray Energy Spectrometry, Ana1. Chem. 45, pp. 604A-614A (1973).

7. F. S. Goulding, J. M. Jaklevic, B. V. Jarrett, and D. A. Landis, Detector Background and Sensitivity of Semiconductor $X$-ray Fluorescence Spectrometers, pp. 470-482, in Ref. 4.

8. E. L. Kothny, Private communication, Calif. Dept. of Publ. Health, Berkeley, CA (1973).

\section{THE REMOTE DETECTION OF POLLUTANTS USING RESONANCE RAMAN SCATTERING*}

H. Rosen, P. Robrish, O. Chamberlain

\section{INTRODUCTION}

This research project is directed toward the development of a new analytical technique for remote, continuous monitoring of pollutant levels in ambient air and from localized sources such as smokestacks. The technique is based on spectroscopic analysis of laser light scattered from the atmosphere (Lidar). By directing a laser beam into the atmosphere and measuring the Raman backscattering, it is possible, in principle, to make a three dimensional map of the concentrations of various molecular constituents. One can isolate the backscattered radiation from a specific smal1 volume in the atmosphere (of the order of $10 \mathrm{~m}^{3}$ ) by requiring that the scattered radiation reach the detector after a time delay with respect to the laser pulse equal to the round-trip flight time of the light to the region of interest. Molecular species can be identified by measuring Raman scattering from the atmosphere, since each molecular species has a characteristic Raman spectrum. 
Raman Lidar systems have been used for some ime to study major atmospheric constituents. 1 However, the fact that Raman cross sections are quite small $\left(-10^{-30} \mathrm{~cm}^{2} / \mathrm{sr}\right)$ has made this technique difficult to use to detect minor atmospheric constituents such as pollutants. In spite of this difficulty, S. Nakahara et a..2 and Melfi et al. 3 have reported detection of $\mathrm{SO}_{2}$ in power plant stack plumes at a range of $200 \mathrm{~m}$ at night, and Hirschfeld et a1. 4 using a powerful doubled ruby laser have been able to measure $\mathrm{SO}_{2}$ concentrations of $30 \mathrm{ppm}$ at $200 \mathrm{~m}$ in full daylight with a good signa1-tonoise ratio.

It is well known that Raman cross sections are enhanced if the laser source is tuned close to an absorption line in a gas. Such enhancements could dramatically increase the sensitivity of a Raman Lidar system. During the past year, we used a narrow-band dye laser $(0.01 \AA)$ to investigate the magnitude of these enhancements in $I_{2}$ vapor and $\mathrm{NO}_{2}$. These measurements, along with recent measurements ${ }^{5}$ of large enhancements in $\mathrm{SO}_{2}$ and calculations of resonance Raman cross sections, were used to estimate the sensitivity of a Lidar system based on the resonance Raman effect. 6

\section{RESONANCE RAMAN SCATTERING IN $\mathrm{I}_{2}$}

We chose to investigate resonance Raman scattering in $I_{2}$ vapor for our initial experiments. There have been several studies 7 of resonance enhancements in iodine using the accidental coincidences between $\mathrm{I}_{2}$ absorption 1 ines and argon and krypton laser wavelengths. These studies have given inconsistent results for the magnitude of the enhancements as we1l as for the quenching of the scattering with the addition of nitrogen. There has also been considerable debate on the distinction between resonance Raman scattering and fluorescence in gases. 7,8 It has been suggested that these are two separate quantum mechanical processes. Recently, Williams et a1.7 argued that fundamentally there is only one process involved, resonance Raman scattering, which has different properties on and off resonance and that these properties change in a continuous fashion as the exciting source is tuned across an absorption line in a gas. The measurements we have made support this point of view. 8

We investigated resonance Raman scattering in $I_{2}$ vapor for several of the strongest rotational absorption 1 ines in the $0-25$ vibrational band of the $B-X$ electronic transition with a narrow-band pulsed dye laser. 9 We chose to investigate this band because the product of the matrix elements for absorption and inelastic re-emission is the largest of the B-X system. Our effort was concentrated on the $0-25 \mathrm{P}(47)$ rotational line which is relatively isolated from other absorption lines. For this line we measured the resonance Raman cross section versus nitrogen buffer gas pressure for various laser frequencies.

In Fig. 1 we show a plot of the integrated intensity of the first Raman mode of $\mathrm{I}_{2}$ versus laser frequency. The spectrum was taken by centering the spectrometer bandpass $\left(75 \mathrm{~cm}^{-1}\right)$ on the Stokes Raman mode at $\sim 213 \mathrm{~cm}^{-1}$ and then tuning the laser. The sample cell contained $\mathrm{I}_{2}$ at its

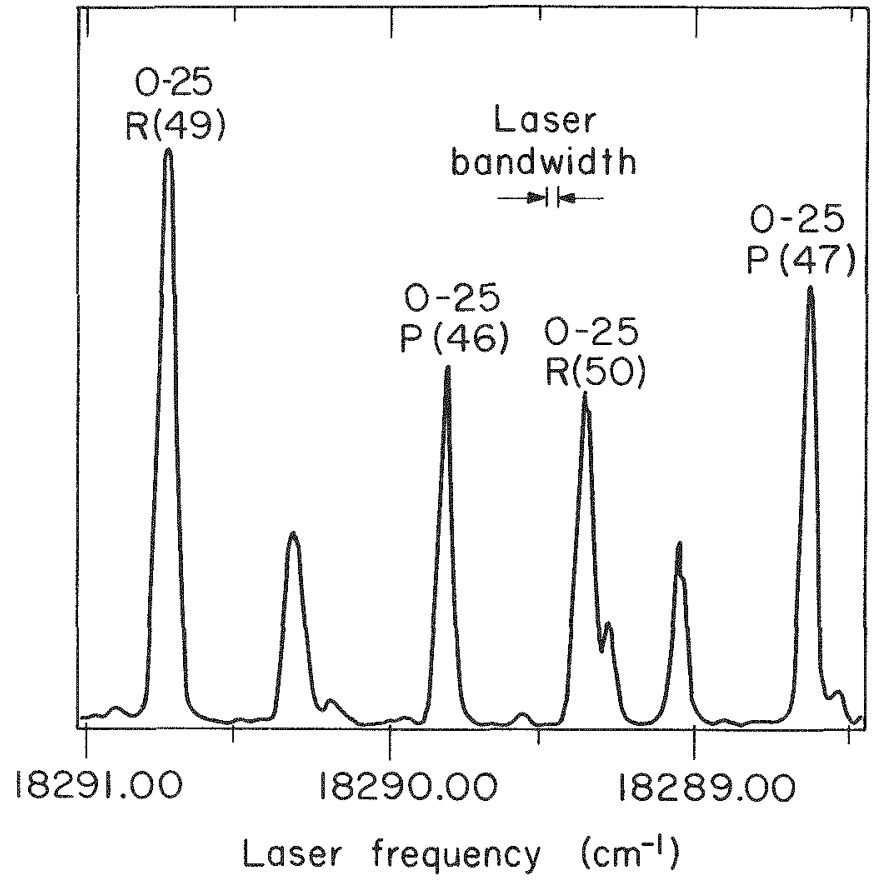

Fig. 1. Scattered intensity of Raman mode located at $\sim 213 \mathrm{~cm}^{-1}$ vs. laser frequency for $\mathrm{I}_{2}$ vapor at a pressure of $0.28 \mathrm{mn}$ with no buffer gas present.

(XBL 743-2658)

room temperature vapor pressure $(0.28 \mathrm{~mm})$ with no buffer gas present. The laser beam was attenuated and defocussed sufficiently to avoid saturation and heating effects. The peaks in the scattered intensity correspond within experimenta1 error to peaks in the absorption spectrum. As one approaches an absorption 1ine, the cross section increases dramatically. For example, at the peak of the 0-25 P(47) transition, the differential scattering cross section, $(\mathrm{d} \sigma / \mathrm{d} \Omega)$, was measured to be $8.3 \times 10^{-21} \mathrm{~cm}^{2} / \mathrm{sr}$ which is ten orders of magnitude greater than the Raman cross section for nitrogen at $5460 \AA$. The cross sections presented here were calculated by using the cross section for the $992 \mathrm{~cm}^{-1}$ mode of benzene as a standard and making sma11 corrections for absorption and spectral efficiency.

We have investigated the quenching of the scattering cross section by nitrogen near the $0-25 \mathrm{P}(47)$ transition. This transition, in contrast to the $0-43 \mathrm{P}(12), \mathrm{R}(14)$ transitions investigated by previous authors, 7 has very little structure within $0.4 \mathrm{~cm}^{-1}$ of its high-frequency side (see Fig. 1). In Fig. 2 we show a plot of the resonance Raman cross section versus nitrogen pressure for several different laser frequencies. For al1 measurements, both the incident laser beam and the scattered light accepted by the spectrometer were polarized perpendicular to the entrance slit. The bandpass of the spectrometer was set to $75 \mathrm{~cm}^{-1}$. With the laser set on resonance, the cross section is quenched by over a factor of 1000 for a change in $\mathrm{N}_{2}$ pressure from $2 \mathrm{~mm}$ to $730 \mathrm{~mm}$. As one tunes off resonance, the quenching becomes progressively smaller until at $0.2 \mathrm{~cm}^{-1}$ away from the absorption line there is virtually no quenching. 


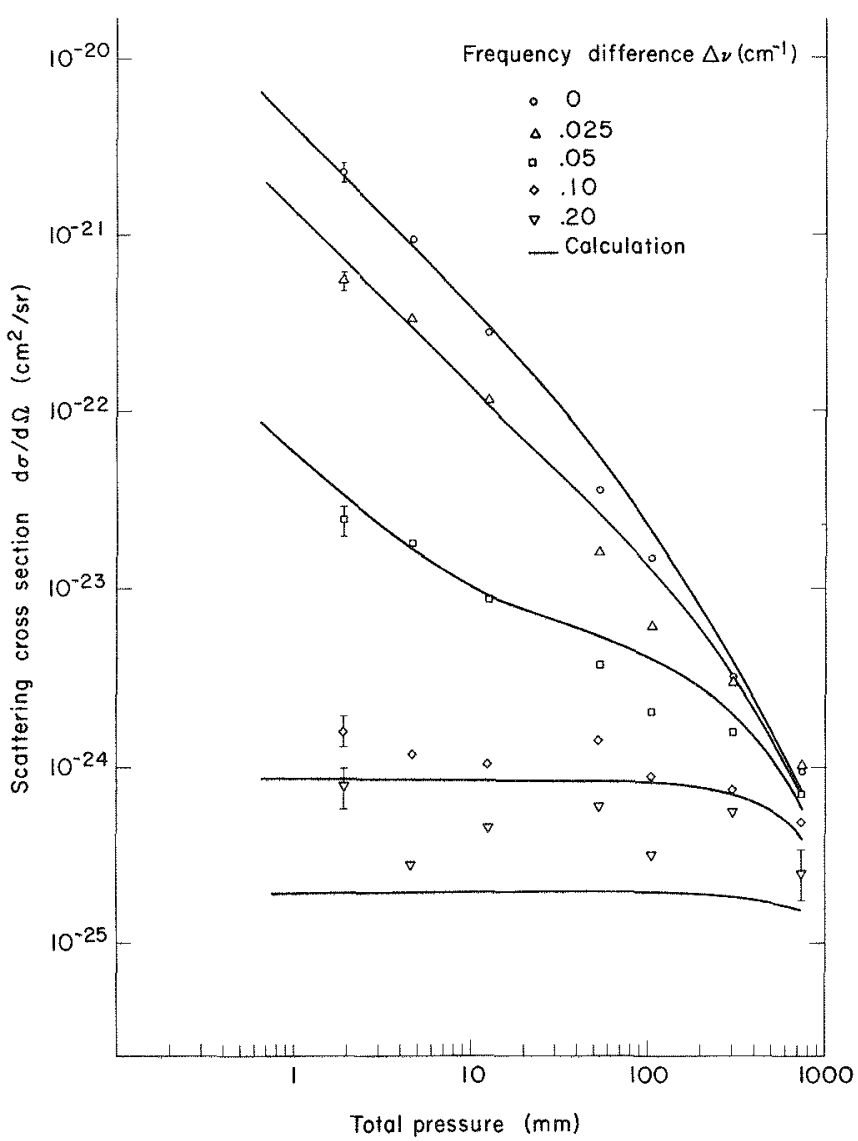

Fig. 2. P1ot of the differential resonance Raman scattering cross section for the Raman mode located at $213 \mathrm{~cm}^{-1}$ vs. nitrogen buffer gas pressure for laser frequencies separated from the center of the $0-25 \mathrm{P}(47)$ rotational line by $0,0.025,0.05,0.1$ and $0.2 \mathrm{~cm}^{-1}$.

(XBL 743-2659)

We can make a quantitative comparison of our data to theory, but the analysis is complicated by the Doppler and hyperfine broadening of the absorption line and the linewidth of the laser. Near an isolated absorption line, the resonance Raman cross section in the impact approximation can be written as: 10

$\frac{d \sigma}{d \Omega} \propto \frac{\Gamma_{T}}{\Gamma_{T}-\Gamma_{e}} \int_{0}^{\infty} S\left(\nu_{\ell}-v_{\ell}^{0}\right) d \nu_{\ell} \int_{0}^{\infty} \frac{G\left(\nu_{r}-\nu_{r}\right) d \nu_{r}}{\left(\nu_{\ell}-\nu_{r}\right)^{2}+\Gamma_{T}^{2}}$

where $G\left(\nu_{r}-v_{r}^{0}\right)$ is the line shape of the resonance due ${ }^{r}$ to Doppler and hyperfine broadening centered at $\nu_{r}^{0}$ and $S\left(v_{\ell}-v_{l}^{0}\right)$ is the line shape of the laser centered at $\nu_{\ell}^{0} \cdot \Gamma_{\mathrm{T}}$ is the linewidth due to collisions and naturâl damping and $\Gamma_{e}$ is the contribution to the line width from elastic collisions. In order to evaluate the dependence of $(\mathrm{d} \sigma / \mathrm{d} \Omega)$ on pressure, $\mathrm{P}$, and $\Delta \nu=\nu_{0}^{0}-\nu_{r}^{0}$, we assume that $G$ and $S$ are Gaussian and use Fourier transforms to reduce the double integral to a single integral. We then neglect natural damping, assume the $\Gamma$ 's vary linearly with $P$, and evaluate the integral numerically as a function of $\Delta v$ and P. The results of such a calculation are shown as the curves in Fig. 2, where we have assumed that $\Gamma_{\mathrm{T}}=\left(1.4 \times 10^{-4} \mathrm{~cm}^{-1} / \mathrm{mm}\right) \times \mathrm{P}$ and that the convoluted Gaussian half-width (at 1/e) of the laser and the resonance is $0.024 \mathrm{~cm}^{-1}$. Over all normalization was set by a best fit to the $\Delta v=0$ data. It is clear that this model calculation adequately represents the data as a function of $\Delta \nu$ and $P$, supporting the view that there is no fundamental distinction between resonance Raman scattering and fluorescence in gases.

\section{RESONANCE RAMAN SCATTERING IN NO 2}

Unlike $\mathrm{I}_{2}$, the spectroscopy of $\mathrm{NO}_{2}$ is very poorly understood and one cannot determine, a priori, the laser frequency which will give the Iargest resonance enhancenents. The absorption spectrum extends from about 2500 to $6500 \AA 11$ and appears to be a broad continuum with some sharp structure superimposed upon it.12 Since we expect to find large enhancements associated with sharp absorption lines whose width is the order of $0.1 \AA$, a complete investigation of $\mathrm{NO}_{2}$ at wavelengths longer than its dissociation limit $(\sim 4000 \mathrm{~A})$ would involve taking spectra at roughly 25,000 laser frequencies. This would be an immense undertaking. Our approach to finding the largest Raman cross section was to concentrate on the $v_{2}$ Raman mode and measure its intensity variation as a narrowband laser $(0.1 \mathrm{~A})$ was continuously scanned thru the $\mathrm{NO}_{2}$ absorption spectrum.

We have scanned our laser from 4200 to $6000 \AA$ and found several narrow spectral regions in which the $\nu_{2}$ Raman mode showed a large enhancement. 13 These regions, which contain sharp and intense lines, are shown in Fig. 3. By narrowing the laser bandwidth to $0.01 \AA$, the 1 ines within a region may be more clearly resolved (see Fig. 4). With the laser tuned to one of the stronger 1 ines near $4546 \AA$, we have measured a cross section of $5.6 \times 10^{-27} \mathrm{~cm}^{2} /$ ster in the presence of one atmosphere of nitrogen. This cross section is about $10^{4}$ times larger than typical Raman cross sections.

\section{FEASIBILITY OF REMOTE DETECTION OF POLLUTANTS}

We have assessed the implications of measurements of large resonance Raman cross sections in $\mathrm{NO}_{2}^{13}$ and $\mathrm{SO}_{2}^{5}$ for remote detection of these pollutants in the atmosphere by means of a Lidar system. We have also made calculations of the resonance Raman cross sections of various diatomic molecules and used these calculations to exoand our estimates of the sensitivity of such a system.

One can estimate the resonance Raman cross sections for diatomic molecules which have known oscillator strengths and Franck-Condon factors. The details 6 of the calculations are not presented, but Table 1 shows the results along with experimentally observed cross-sections.

Using the cross sections for $\mathrm{NO}_{2}, \mathrm{SO}_{2}$, and NO from Table 1, we have made a calculation "of the sensitivity of a model Lidar system. The laser transmitter in this model system has an energy of $0.05 \mathrm{~mJ} /$ pulse and a repetition rate of $100 \mathrm{~Hz}$. 

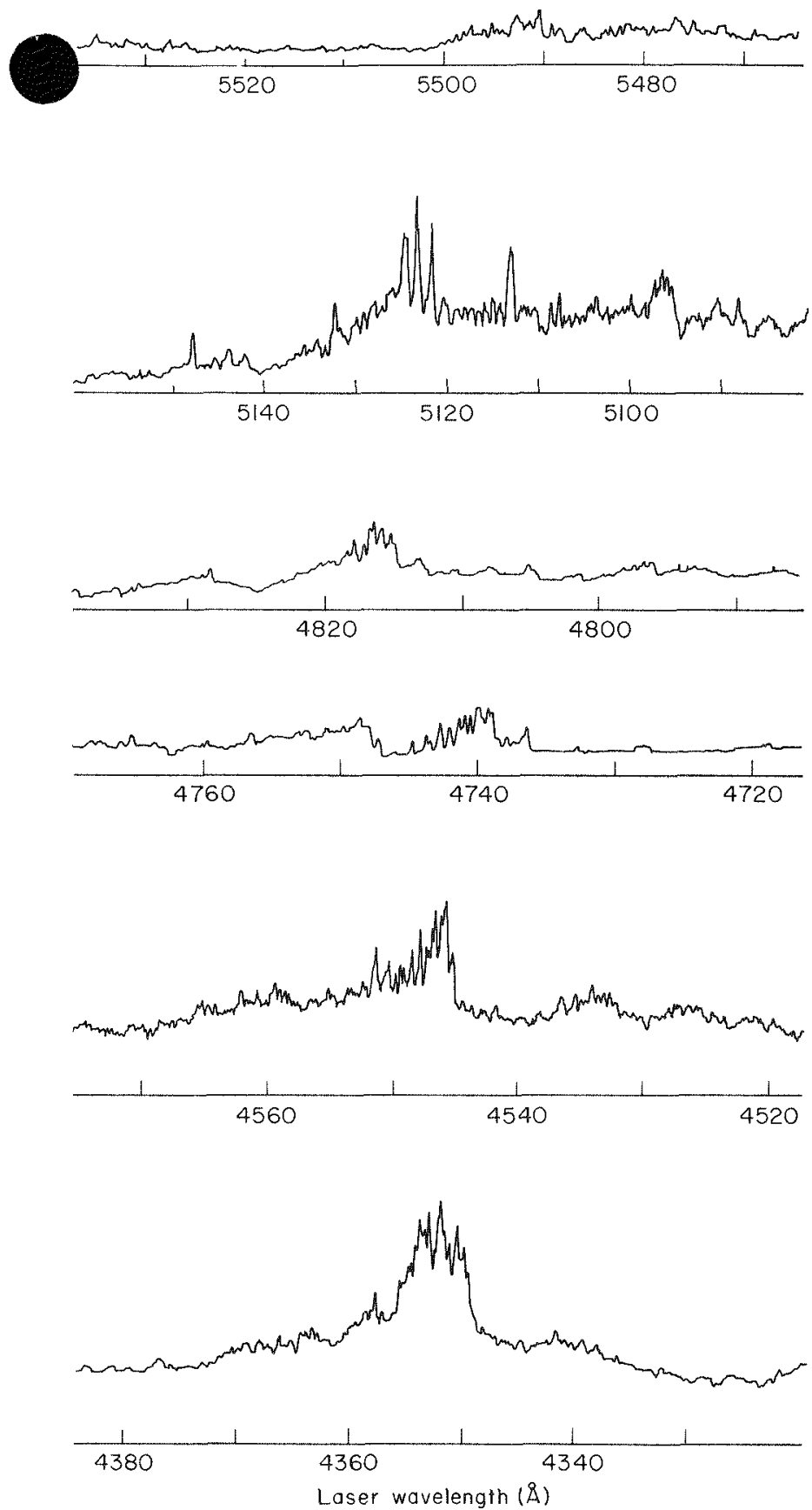

Fig. 3. Intensity in the $v_{2}$ mode as a function of laser wavelength for wavelength regions in which sharp and intense structures were observed. The bandwidth of the laser was 0.1 and the spectrometer bandwidth was set to $25 \AA$ The intensities in different regions cannot be directly compared due to variations in laser power and detector sensitivity.

(XBL 7412-8438)

he laser power level was chosen to meet the visble eye safety standard for direct irradiance. the receiver had characteristics comparable with those of presently operating systems. Figure 5 shows the results of a calculation of the pollutant concentration which can be measured to $10 \%$ accuracy in 100 seconds with a range resolution of 10 meters.

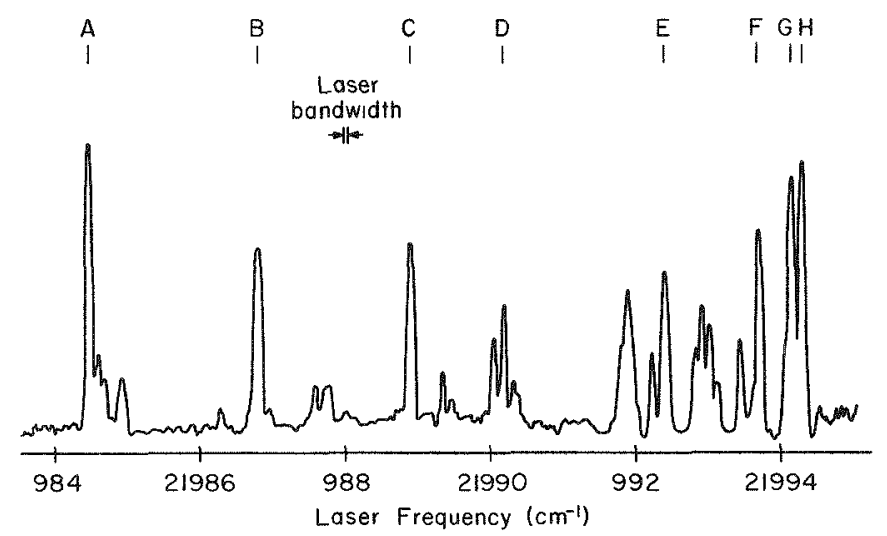

Fig. 4. Intensity in the $v_{2}$ mode as a function of laser frequency near $22,000 \mathrm{~cm}^{-1}(4545 \AA)$ with narrowband laser excitation. The Iinewidth of the laser was $0.04 \mathrm{~cm}^{-1}$ and the bandwidth of the spectrometer was $120 \mathrm{~cm}^{-1}$.

(XBL 7412-8437)

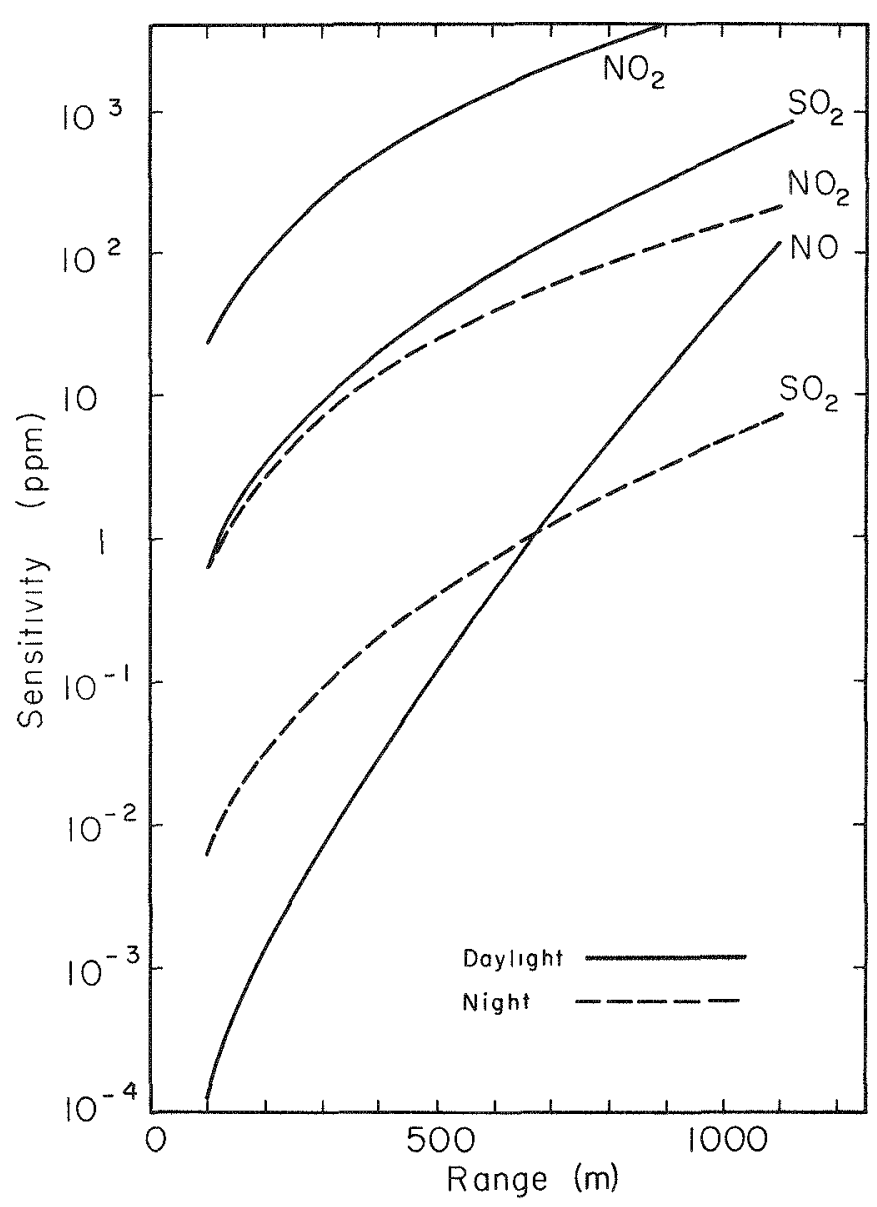

Fig. 5. Lidar sensitivity vs. range for $E=0.05 \mathrm{~mJ}$ and $N=10^{4}$ pulses assuming a signal to noise ratio of 10 and a visibility of $19 \mathrm{~km}$.

(XBL 748-3947)

Figure 5 shows that, with the marginal exception of daylight detection of $\mathrm{NO}_{2}$, a Lidar system making use of resonance Raman scattering can easily detect the pollutant concentrations 
Table 1. Resonance Raman cross sections for various small molecules determined or from calculation in LBL-3215.

\begin{tabular}{|c|c|c|c|c|c|c|c|}
\hline \multicolumn{2}{|l|}{ Molecule } & \multicolumn{2}{|c|}{$\begin{array}{l}\text { Electronic } \\
\text { Transition }\end{array}$} & \multirow{2}{*}{$\begin{array}{c}\begin{array}{c}\text { Vibrational } \\
\text { Transition }\end{array} \\
0-1,1-1\end{array}$} & \multirow{2}{*}{$\frac{\lambda_{\mathrm{L}}(\AA)}{4913}$} & \multirow{2}{*}{$\frac{\frac{\mathrm{d} \sigma}{\mathrm{d} \Omega}\left(\mathrm{cm}^{2} / \mathrm{ster}\right) \text { CALC. }}{2.5 \times 10^{-20}}$} & \multirow[t]{2}{*}{$\frac{\mathrm{d} \sigma}{\mathrm{d} \Omega}\left(\mathrm{cm}^{2} / \text { ster }\right)_{\text {EXP. }}$} \\
\hline$\overline{\mathrm{CO}^{+}}$ & A & $2^{2} i$ & $-x^{2} \Sigma^{+}$ & & & & \\
\hline So & A & $3_{\Pi_{0}}$ & $-x^{3} \Sigma^{-}$ & $0-2,2-0$ & 2579 & $1.3 \times 10^{-20}$ & \\
\hline $\mathrm{OH}$ & A & $2_{\Sigma}^{+}$ & $-x^{3} i$ & $0-0,0-1$ & 3064 & $2.4 \times 10^{-22}$ & $\sim 10^{-20}-10^{-21}$ \\
\hline NO & $\mathrm{A}$ & ${ }^{2} \Sigma^{+}$ & $-x^{2} \Sigma$ & $0-0.0-3$ & 2262 & $1.3 \times 10^{-23}$ & \\
\hline $\mathrm{I}_{2}$ & B & $3_{\mathrm{II}}^{+}$ & $-x^{1} \Sigma_{g}^{+}$ & $0-25,25-1$ & 5466.36 & $1.2 \times 10^{-24}$ & $1.7 \times 10^{-24}$ \\
\hline $\mathrm{SO}_{2}$ & A & $\left({ }^{1} \mathrm{~B}_{1}\right)$ & $-x^{1} A_{1}$ & & 3002 & & $1 \times 10^{-25}$ \\
\hline${ }^{79} \mathrm{Br}{ }^{81} \mathrm{Br}$ & B & $3_{\mathrm{II}}^{+}$ & $-x^{1} \Sigma_{\mathrm{g}}^{+}$ & $0-28,28-1$ & 5264.0 & $7.8 \times 10^{-27}$ & \\
\hline $\mathrm{NO}_{2}$ & A & $\left({ }^{2} B_{1}\right)$ & $-x^{2} A_{1}(?)$ & & 4547.4 & & $5.6 \times 10^{-27}$ \\
\hline $\mathrm{CO}$ & a & $3_{\pi}$ & $-x^{1} \Sigma^{+}$ & $0-0,0-1$ & 2063 & $4.5 \times 10^{-30}$ & \\
\hline $\mathrm{Cl}_{2}$ & A & $3_{\mathrm{II}}^{+} \mathrm{Ou}$ & $-x^{1} \Sigma_{g}^{+}$ & $0-15,15-3$ & 4933 & $7 \times 10^{-31}$ & \\
\hline
\end{tabular}

typical of smoke plumes (>10 ppm) at a range of a few hundred meters using lasers with only very modest energies. A useful comparison can be made with the recent report 4 of detection of $\mathrm{SO}_{2}$ using ordinary Raman scattering. In that case, the laser used had an average power of 0.4 watts and an energy/pulse of 0.2 joules, and was able to detect $30 \mathrm{ppm}$ at $200 \mathrm{~m}$ with SNR $\sim 10$. Our calculation shows that using a laser of $5 \mathrm{~mW}$ average power and $0.05 \mathrm{~mJ} /$ pulse, one could achieve a sensitivity of a factor of 10 better by making use of the resonance Raman effect.

Monitoring of ambient concentrations $(\sim 0.1 \mathrm{ppm})$ at distances of order $1 \mathrm{Km}$ (see Fig. 5 ) is certainly out of the question, given the safety constraint imposed on the calculation. If we can be sure that there will be no eye exposure to the light from the Lidar transmitter, then we might be able to use high-energy tunable lasers to gain the required sensitivity.

In conclusion, it seems to us that the advantages in sensitivity and safety associated with the use of resonance enhancements in a Raman Lidar system outweigh the additional problems associated with the use of a tunable laser as a Lidar transmitter. The fact that an instrument for use in populated areas will probably have to comply with stringent eye safety standards implies that the use of resonance enhancements will be the only avenue to a workable Raman Lidar system for remote smokestack monitoring.

\section{Footnote and References}

* Research supported in part by NSF/RANN.
1. D. A. Leonard, Nature 216, 142 (1967). J. A. Cooney, App. Phys. Let 12,40 (1968). T. Kobayasi and H. Inaba, Proc. I.E.E.E., 58, 1968 (1970). S. H. Melfi, J. K. Lawrence, Jr., and M. P. McCormick, App1. Phys. Lett. 15, 295 (1969)

2. S. Nakahara, K. I to, S. I to, A. Fuke, S. Komatsu, H. Inaba, and T. Kobayasi, Opto-electronics $\underline{4}$, $169(1972)$

3. S. H. Melfi, M. L. Brumfield, and R. W. Storey, Jr., App. Phys. Lett. 22, 402 (1973).

4. T. Hirschfeld, E. R. Schildkraut, H. Tannenbaum, and D. Tannenbaum, App. Phys. Lett. 22, 38 (1973).

5. C. M. Penney, W. W. Morey, R. L. St. Peters, S. D. Silverstein, M. Lapp, and D, R. White, N.A.S.A. Report number NASA-CR-132363.

6. H. Rosen, P. Robrish and O, Chamberlain, LBL3215 (to be published).

7. P. F. Williams, D. L. Rousseau and S. H. Dworetsky, Phys. Rev. Lett. 32, 196 (1974). D. G. Fouche and R. K. Chang, Phys. Rev. Lett. 29, 536 (1972). R. L. St. Peters, S.D. Silverstein, M. Lapp, and C. M. Penny, Phys. Rev. Lett. 30, 191 (1973).

8. P. Robrish, H. Rosen, and O. Chamberlain, Phys. Lett. 51A, 434 (1975).

9. T. W. Hansch, App. Opt. 11, 895 (1972).

10.D. L. Huber, Phys. Rev, $\overline{178}, 93$ (1969).

11.T. C. Hal1, Jr., and F. E. Blacet, J. Chem. Phys. 20, 1745 (1952).

12. A. E. Douglas and K. P. Huber, Can. J. Phys. 43,74 (1965).

13. $\overline{\mathrm{P}}$. Robrish and H. Rosen, LBL-3229 (to be published). 


\section{INTRODUCTION}

The use of cholesteric liquid crystals as sensitive gas detectors is being investigated for air pollution monitoring applications. In preliminary studies using a simple prototype, we have achieved sensitivities of better than $10^{-5}$ for certain organic gases.

Liquid crystals are fluids with anisotropic properties sometimes known as "mesophases". They are equilibrium phases with well defined latent heats of transition. Liquid crystals are made of elongated organic molecules whose general formula is

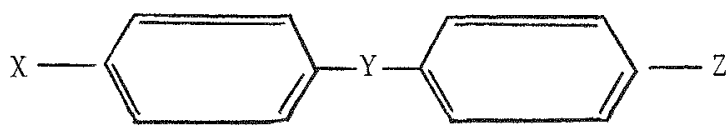

Typically $\mathrm{Y}$ is $-\mathrm{NO}=\mathrm{N}-,-\mathrm{N}=\mathrm{N}-$, or $-\mathrm{CO}-\mathrm{O}-$; and the flexible tails $X$ and $Z$ are $-0-C_{m} H_{2 m+1}$ or $-\mathrm{C}_{\mathrm{n}} \mathrm{H}_{2 \mathrm{n}+1}$.

Based on molecular arrangement, three classes of 1iquid crystals exist:

(1) In the nematic phase, the centers of gravity of the molecules are arranged more or less randomly, except for a degree of short range order. While the molecules fluctuate rapidly, as in isotropic 1iquids, their axes have an average direction which is well defined in a macroscopic region.

(2) In the smectic phases, the molecules are packed parallel to each other in successive equidistant planes.

(3) The cholesteric phase can be thought of as derived from a nematic phase by a static torsion with an axis perpendicular to the molecules. This helical arrangement is related to some skew character of the molecules. The helical arrangement has a period equal to half its pitch. The pitch may vary from 0.2 to $20 \mu$. Bragg reflection of visible light from such periodic structures gives rise to spectacular iridescent colors.

Interest in the liquid crystalline phase has been revived because of the small energies involved in their ordering. They are easily perturbed by temperature, thermal gradient, electric fields and currents, magnetic fields, flow, and boundary conditions. In the case of cholesteric liquid crystals, the pitch is sensitive to small traces of vapors in the environment. The change in pitch is manifested as a change in the wavelength of the Bragg reflected 1ight. This property is ultilized to study the feasibility of detecting

$$
\text { vall amounts of gases. }
$$

\section{XPERTMENTAL ARRANGEMENT AND PRELIMINARY RESULTS}

The experimental setup is shown in Fig. 1. A copper heat sink provided uniform temperature across the substrate supporting the cholesteric

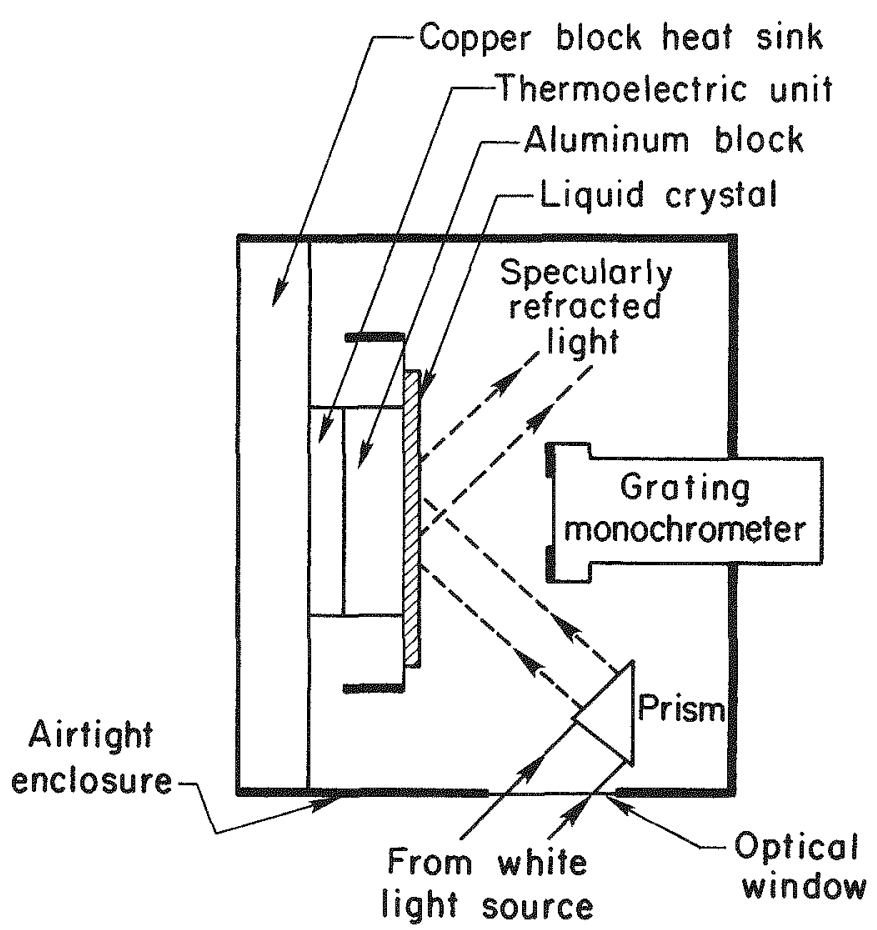

liquid crystal film. A $0.25 \mathrm{~m}$ grating monochromator is used in conjunction with a photomultiplier (pm) tube to analyze the light scattered from the liquid crystal at a fixed angle with respect to the incident lights. The whole assembly is mounted in an air-tight box.

The experimental procedure is as follows: The air-tight box is evacuated and the temperature of the liquid crystal stabilized. A reading of the wavelength of the scattered light is then taken. By means of a needle valve, known concentrations $\left(10^{-4}, 10^{-5}, 10^{-6}\right)$ of chloroform, methylene, in nitrogen are introduced inside the box. A summary of the results is shown in Table $I$. It can be

TABLE I .

\begin{tabular}{|c|c|c|c|}
\hline $\begin{array}{l}\text { Test } \\
\text { Gas }\end{array}$ & $\begin{array}{l}\text { Concen- } \\
\text { tration }\end{array}$ & $\begin{array}{l}\text { Selectively } \\
\text { reflected } \\
\text { wavelengths } \\
\text { in absence } \\
\text { of gas }(\AA)\end{array}$ & $\begin{array}{l}\text { Selectively } \\
\text { reflected } \\
\text { wavelength } \\
\text { when gas is } \\
\text { present }(\AA)\end{array}$ \\
\hline Chloroform & $\begin{array}{l}10^{-4} \\
10^{-5} \\
10^{-6}\end{array}$ & 6228 & $\begin{array}{l}5115 \\
5415 \\
5928\end{array}$ \\
\hline Trichlorobenzene & $\begin{array}{l}10^{-4} \\
10^{-5} \\
10^{-6}\end{array}$ & 6228 & $\begin{array}{l}5970 \\
5625 \\
6100\end{array}$ \\
\hline $\begin{array}{l}\text { Methylene } \\
\text { Chloride }\end{array}$ & $\begin{array}{l}10^{-4} \\
10^{-5} \\
10^{-6}\end{array}$ & 6228 & $\begin{array}{l}5025 \\
5615 \\
6035\end{array}$ \\
\hline
\end{tabular}


seen that the wavelength of the scattered light shifted considerably; this shift is easily detected with the naked eye. When the box is reevacuated, the wavelength returns to its original value. Cycling the system several times did not show any hysteresis.

These results demonstrate that cholesteric liquid crystals can be used as sensitive and inex- pensive detectors for certain organic vapors. Work is now underway to extend this method to other gases and to obtain additional information requi for the development of an instrument suitable for routine air pollution applications.

\section{Footnote}

* Condensed from LBL-3088.

DIRECT FLAMELESS ATOMIC ABSORPTION ANALYSIS OF SAMPLES WEIGHING SEVERAL TENTHS OF A GRAM*

T. Hadeishi and R. D. McLaughlin

Isotope-shift Zeeman-effect Atomic Absorption (IZAA) spectroscopy has been developed as an effective analytical tool over the last two years. This technique utilizes a Zeeman split-light source to perform atomic absorption ana1ysis. I The Zeeman components that are shifted away from the resonant analytical line are used to correct for light losses that are caused by processes other than absorption by the element of interest. This technique allows mercury to be determined at the one hundredth of a ppm level by passing the light through the smoke and vapors that are given off when biological samples (e.g. tuna fish, bovine liver, orchard leaves) are thrust into a furnace whose temperature is about $900^{\circ} \mathrm{C} .2$

The application of this technique to the determination of cadmium and lead resulted in an interesting discovery. If large volumes of solution are inserted into the furnace, the water is rapidly vaporized leaving behind the lead or cadmium. Whereas the water vapor shoots through the furnace in two or three seconds, the lead or cadmium requires 30 to 60 seconds for passage (depending upon the furnace temperature). We thus have a one stage gas chromatograph that is capable of performing a metal separation and determination in less than one minute.

An understanding of the mechanism of this separation may be found by referring to Fig. 1. The vaporization occurs inside a probe that is inserted into the side arm of the furnace. The vapors are forced from the probe into a large volume surrounding the cylinder through which the light beam is absorbed. They then flow through small holes into the absorption region. A flowing stream of hydrogen insures that the metal salts that result from this treatment will be converted to the atomic state and then these atoms will exit by passing through the absorption region. Separation occurs because of the large differences in volatility under these conditions. The volatization chamber is constructed from quartz and is heated by silicon carbide heating elements. Although this design should allow the use of temperatures higher than $1400^{\circ} \mathrm{C}$, there is no need to exceed $1100^{\circ} \mathrm{C}$ for cadmium and lead determinations.

This separation technique will only be effective if the metal salts can be caused to strike and momentarily stick to the walls of the furnace. As larger and larger volumes of solution are used the probability of interaction with the wa11s becomes

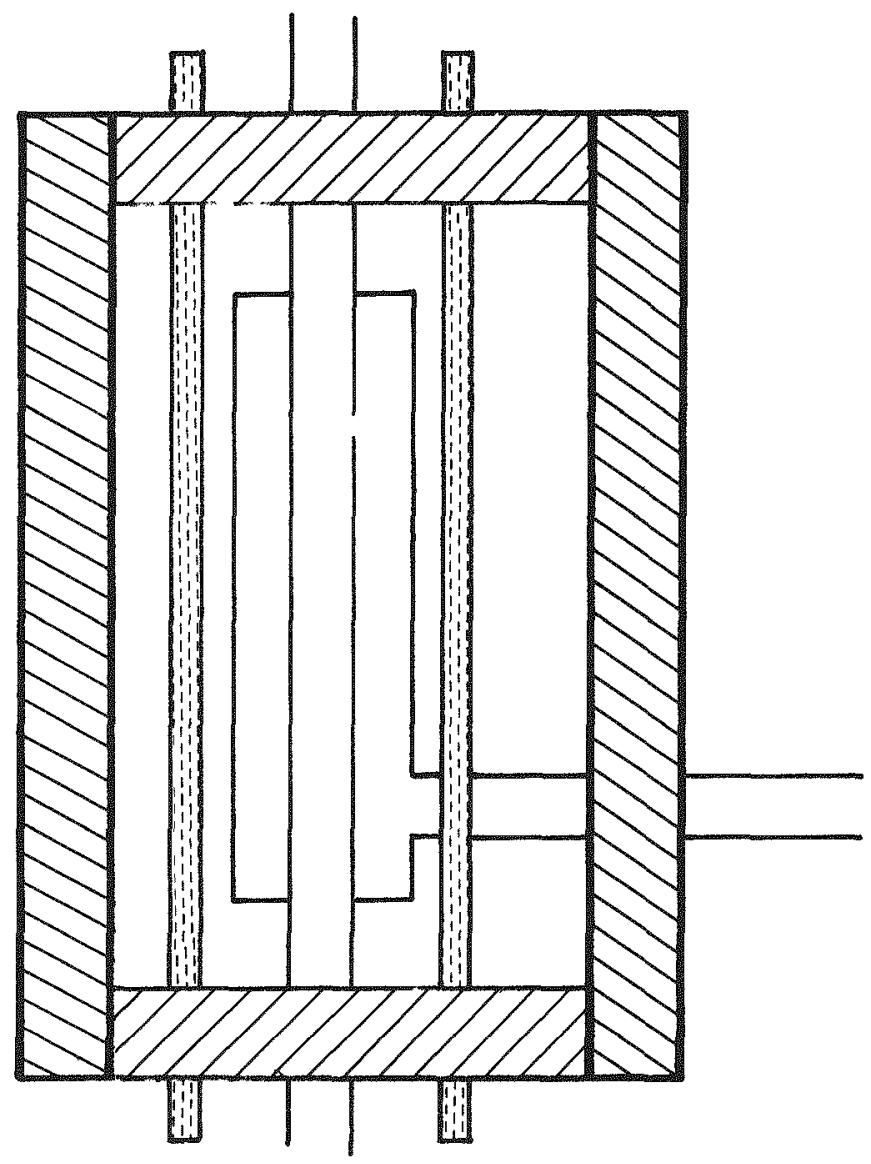

Fig. 1. Furnace design for 1 ead and cadmium determinations.

(XBL 755-2971)

less and 1ess. The present system is capable of separating cadmium salts from $0.5 \mathrm{ml}$ of solution with negligible loss of cadmium. Lead salts are held less tenaciously by the quartz walls and losses occur at somewhat lesser volumes.

This approach can be applied to solid samp by simply placing a known weight of the solid $i$ the probe and adding an equal weight of concentroud nitric acid. The probe containing the mixture is placed under a heat lamp for a few minutes so that partial decomposition of the biological sample occurs. In order to determine the lead or cadmium 
concentration of the sample, the probe is inserted nto the furnace and the signal generated is comred to the signal generated from a reference olution. Experiments are in progress to define the capabilities and limitations of this procedure.

\section{FOOTNOTES AND REFERENCES} Work supported by the National Science FoundationRANN Division and the U.S.E.R.D.A.

1. T. Hadeishi, App1. Phys. Lett. 21, 438 (1972). 2. D. A. Church, T. Hadeishi, L. Leong, R. D. Mclaughlin, and B. D. Zak, Anal. Chem. 46, 1352 (1974).

\section{AN IMPROVED TECHNIQUE FOR THE QUANTTTATIVE ASSAY OF SOILS AND SEDIMENTS FOR CADMTUM}

\section{B. R. Moyer and T. F. Budinger}

Soils and sediments are appropriate environmenta1 monitoring sites for cadmium because they comprise a major sink in the environmental progression of cadmium in the food chain. The increased usage of sewage sludge and phosphate (both high in cadmium) as fertilizers in agricultural soil has necessitated increased monitoring for reasons of public health. Cadmium levels from many agricultural areas show significant elevation from a variety of sources.1

In our study of cadmium in San Francisco Bay sediments ${ }^{2}$ we found that the widely used acid-extraction method of Nakagawa and Harmes ${ }^{3}$ proved to be inadequate for the desired sensitivity and reproducibility. In comparing the results of their analytical procedures with the procedure we describe here for the recovery of added cadmium from soil, we can see a significant loss of true metal levels. The signal attenuation resulting from their method is due principally to the large concentrations of calcium, iron, and sodium (relative to cadmium) as well as the changes in sample viscosity resulting from preconcentration for analysis. Our analytical technique, which is based on previous investigations, 3-10 consists of leaching out acidsoluble cadmium, removing interfering elements by cation-exchange chromatography, and concentrating the cadmium by extraction into an organic phase.

In the method we have developed, $\sim 8 \mathrm{~cm}^{3}$ of soil or sediment sample is dried $\left(110^{\circ} \mathrm{C}\right.$ for $\left.8 \mathrm{hrs}\right)$, crushed, sieved $(<1 \mathrm{~mm})$, and redried $\left(110^{\circ} \mathrm{C}\right.$ for $3 \mathrm{hrs}$ ). Approximately $10 \mathrm{~g}$ of the sample is then leached with a mixture of hydrochloric, nitric, and sulfuric acids (ratio $=10: 7.5: 1$ ). The leaching is accomplished over low heat with stirring until a sufficient loss of liquid makes the sample viscous. The sample is then washed with a dilute (3N) solution of hydrochloric acid in a Buchner funnel until the filtrate is clear. The $\mathrm{pH}$ of the filtrate is adjusted to approximately 1.0. Samples can be analyzed directly at this point, but there is a potential for serious error if other metals such as iron are present.

Cadmiun is separated from the other major metals in soil using cation-exchange chromatography. Bio-Rad $A G-50 W-X 8$, a 50 to 100 mesh resin, is packed in a standard $50 \mathrm{~cm}^{3}$ buret $\left(40 \mathrm{~cm}^{3}\right.$ of resin

ind) and used to elute cadmium quantitatively and

eliminate iron, sodium, and much of the calcium. cadmium in the eluate is then complexed by the addition of a $5 \%$ solution of ammonium pyrrolidine dithiocarbamate and then extracted with water saturated Methyl Isobuty] Ketone (MIBK). This extraction further reduces the concentration of interfering cations. Atomic absorption analysis is performed on the organic phase.

The method described above provides for a substantially improved sensitivity for cadmium. The MIBK solvent provides a uniform sample aspiration, a uniforn field of excited cadmium ions in the beam path, and an increased aspiration rate due a lower solvent surface tension relative to acid/ water mixtures. Figure 1 compares the relative

\section{SIGNAL STRENGTH \\ AQUEOUS vS. ORGANIC MEDIA}

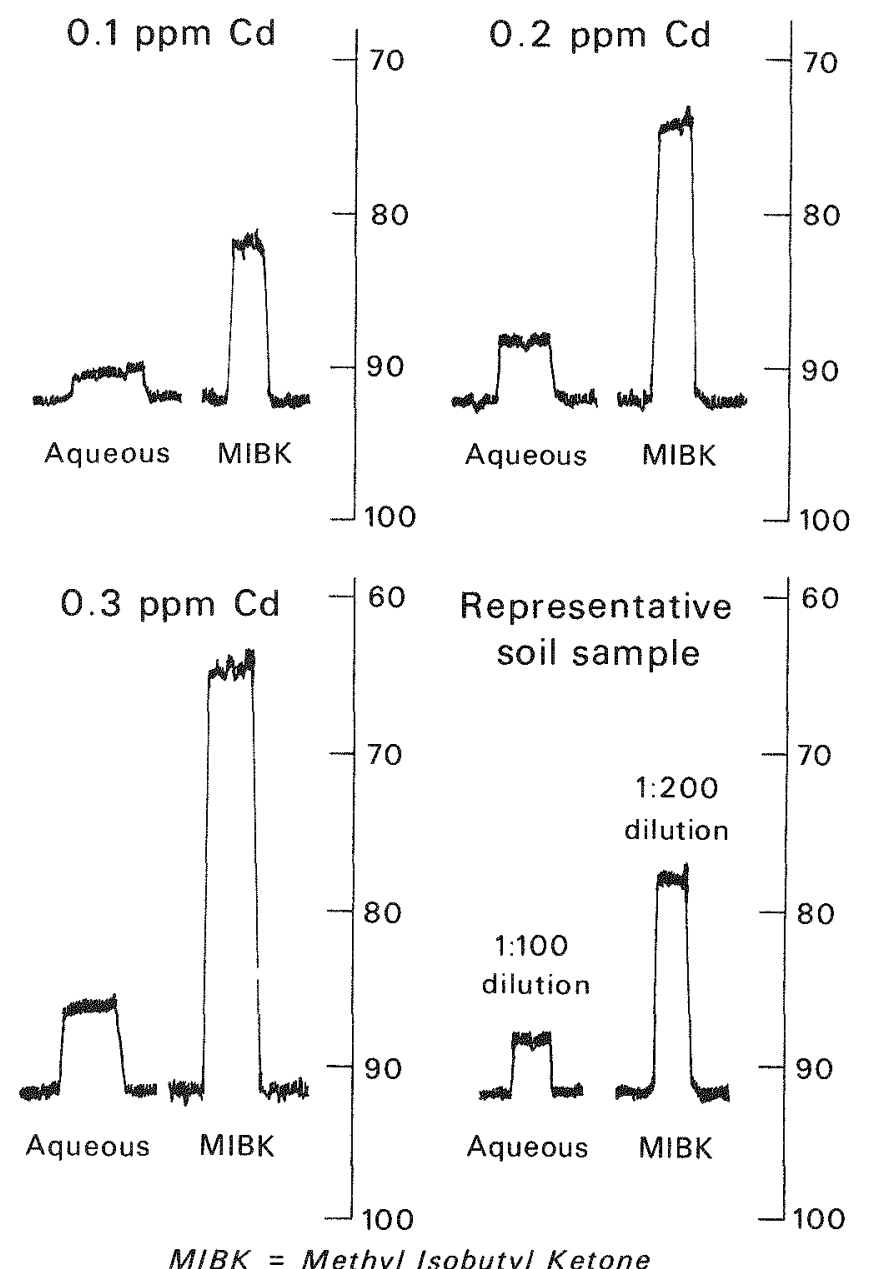

Fig. 1. Comparison of the relative atomic absorption signal strengths for cadmium in aqueous and organic phases.

(XBL, 738-5293) 
signal strengths for aqueous and organic phases and demonstrates the improved sensitivity using the organic phase. The method also eliminates the usual problems of interferences that result from high concentrations of calcium, iron, and sodium relative to cadmium when using a single beam atomic absorption instrument.

\section{FOOTNOTES AND REFERENCES}

1. M. K. John, H. H. Chuah, and C. J. VanLaerhoven, Environ. Sci. Technol. 6, 555 (1972).

2. B. R. Moyer and T. F. Büdinger, Cadmium levels in the shoreline sediments of San Francisco Bay, in Proceedings of the Conference on Trace Substances in Environmental Health, edited by D. D. Hemphill, Univ. of Missouri (1974), pp. 127-135.
3. H. M. Nakagawa and T. F. Harms, U.S. Geol. Survey Prof. Paper 600-D, (1968), pp. 207-209.

4. F. N. Ward, H. M. Nakagawa, T. F. Harms, and G. W. Van Sickle, U.S. Geol. Survey Bull. $1289,(1969)$.

5. J. R. DeVoe, Nat. Acad. Sci. Nuclear Science Series Report NAS-NS-3001, (1960).

6. 0. Samualson, Ion Exchange Separations in Analytical Chemistry, John Wiley \& Sons, New York, 1963 .

7. F. W. E. Strelow, Anal. Chem, 32, 363 (1960).

8. F. W. E. Strelow, A. H. Victor, C. R. Van Zy1 and C. Eloff, Ana1. Chem. 43, 870 (1970).

9. G. Lehnert, R. W. Schaller, and Th. Haas, Z. KIin. Chem. Klin. Biochem. 6, 174 (1968) in German.

10. T. Nagata, H. Shimura, and T. Tirashima, J. Food Hyg. Soc. Japan 11, 41 (1970) in Japanese.

\title{
CAUSE OF LOSS OF HYDROGEN OVERVOLTAGE AT GRAPHITE ELECTRODES USED FOR ANODIC STRIPPING VOLTAMETRY*
}

\author{
Ray G. Clem
}

Anodic Stripping Voltametry (ASV) is an extremely sensitive method for determining the concentrations of certain metals in solution. We have used ASV extensively for the estimation of lead in estuarine waters and sediments. Our work requires the analysis of samples which are acidified to a $\mathrm{pH}>1$ but $\leq 3$. Failures, manifested as a loss of hydrogen overvoltage, of graphite ASV electrodes under these conditions have been a serious problem and have prompted us to investigate the cause of these failures.

Previous workers believed that the cause of failure of graphite ASV electrodes in acid solution was attributable to a solution invasion of the electrode along channels presumed to exist between the graphite particles and the wax impregnator. ${ }^{1}$ However, recent work with a graphite electrode, impregnated with styrene chemically bonded to the graphite by ${ }^{60} \mathrm{Co}$ irradiation, demonstrated conclusively that electrode failure was in some way related to the failure of the graphite itself.2

Two hypotheses were examined for the cause of the graphite failure: 1) the formation of lamellar compounds between the graphite planes and 2) oxidation of the graphite. The first proposed failure mode should be sensitive to structural alterations which seal off or reduce the accessible area between the graphite planes. The second failure mode should be sensitive to chemical conditions (especially the presence of oxidants) in the electrochemical cell.

Structurally-altered graphite electrodes were prepared by irradiation with fast neutrons. Al though irradiation extensively disrupted the planar structure, the electrodes exhibited the same failure behavior as unirradiated graphite. Therefore, we felt that the first hypothesis could not be correct. Mercuric ion and especially $\mathrm{Ag}^{+}$ strongly calalyze the failure of the graphite electrode in acidic solutions. The pressure of $\mathrm{H}_{\mathrm{g}}^{2+}$ is assential to the function of the graphite electrode for ASV applications.
The second hypothesis seemed more likely after it was learned from further experiments that the behavior of electrodes used to the point of failure was the same as that of electrodes that had been treated with oxidizing agents such as $\mathrm{S}_{2} \mathrm{O}=\overline{8}, \mathrm{MnO}_{4}^{-}$, and $\mathrm{Ce}^{4+}$. Further evidence in support of the second hypothesis was obtained using X-ray photoelectron spectroscopy and infrared spectroscopy. X-ray photoelectron spectroscopy of an electrode surface before and after use indicated an increase in oxygen content. Infrared analysis of an electrode used to the point of failure indicated the presence of carboxyl groups on the surface.

A number of attempts were made to restore the oxidized electrodes. Treatment with diazomethane or lithium aluminum hydride resulted in surface derivatives which were easily hydrolyzable or easily reoxidized. Photochemical decomposition of surface carboxyl groups with vacuum ultraviolet light gave an electrode with exceptionally good hydrogen overvoltage characteristics, but one which had virtually no sensitivity to ASB determinable metals. After some use, the hydrogen overvoltage characteristics of the electrode deteriorated and the ASV performance improved.

We conclude that the cause of failure of graphite ASV electrodes is surface oxidation, but that some surface oxidation is required for good ASV performance. Attempts to produce electrodes which will operate reliably under acidic conditions have not yet succeeded. At present, it is desirable, if at all possible, to work in solutions having $\mathrm{a} \mathrm{pH}>3$ and preferably in the $\mathrm{pH}$ interval between 5 and 7 .

\section{FOOTNOTES AND REFERENCES}

*Condensed from LBL-3244.

1. W. R. Seitz, Ph. D. Thesis, M.I.T. (1970) .

2. R. G. Clem, and A. F. Sciamanna, Ana1. Chem. 47, 276 (1975). 
AQUEOUS SULFIDE LEVEL DETERMINATIONS BY SOFT X-RAY FLUORESCENCE ANALYSES

A. J. Hebert

A previously descrabed nondispersive soft $X$-ray fluorescence spectrometer ${ }^{1}$ has been used to determane sulfide levels in natural waters. A technique has been developed that is applicable for solutions with inorganic and organic sulfides in the presence of other species, including sulfate.

The method which has been developed uses a homogeneous group of silverplated copper disks. First, one of the disks is brought into contact with the water sample as shown in Fig. 1. After several hours the sulfide level decreases by several orders of magnitude. The sample is then weighed and used to calibrate the analysis. This is done by "spikıng" with a carefully prepared and measured saturated solution of hydrogen sulfide in water. A second silver disk placed in the spiked sample and left for several hours is analyzed to provide a calibration point.

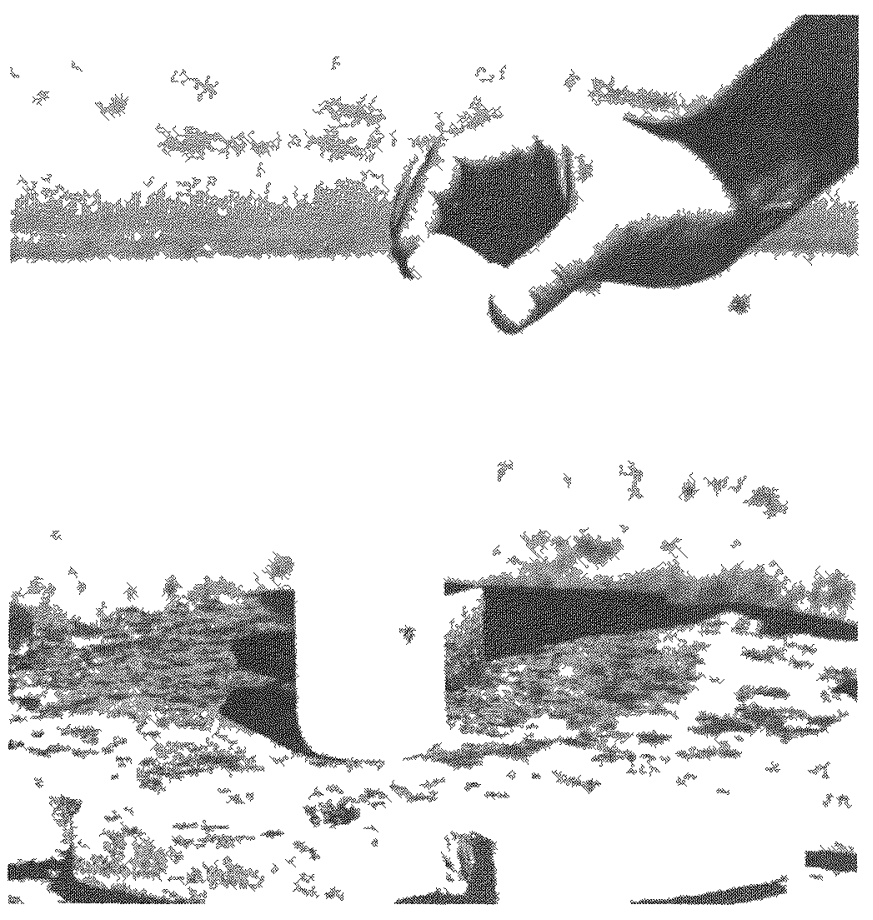

Fig. 1. Method for field monitoring $\mathrm{H}_{2} \mathrm{~S}$ and sulfide levels in waters. A water sample is placed in the plastic bottle and a silver disk is suspended in the water with a plastic rod attached to the bottle cap.
A typical calibration line is shown (F1g. 2) dlong with several observed sulfide levels in natural waters. The calibration figure is obtained by 2 -minute spectrometer runs using a silver anode to generate the exciting radiation.

In studies with natural waters ${ }^{2}$ a difterence in the appearance of the sulfide deposit on the disks is observed when the water has not been filtered through a $0.45 \mu$ fliter as compared to the pattern from the same water after filtration. This may be due to the presence of organic sulfide species. Further studies should lead to a better under standing of this phenomenon.

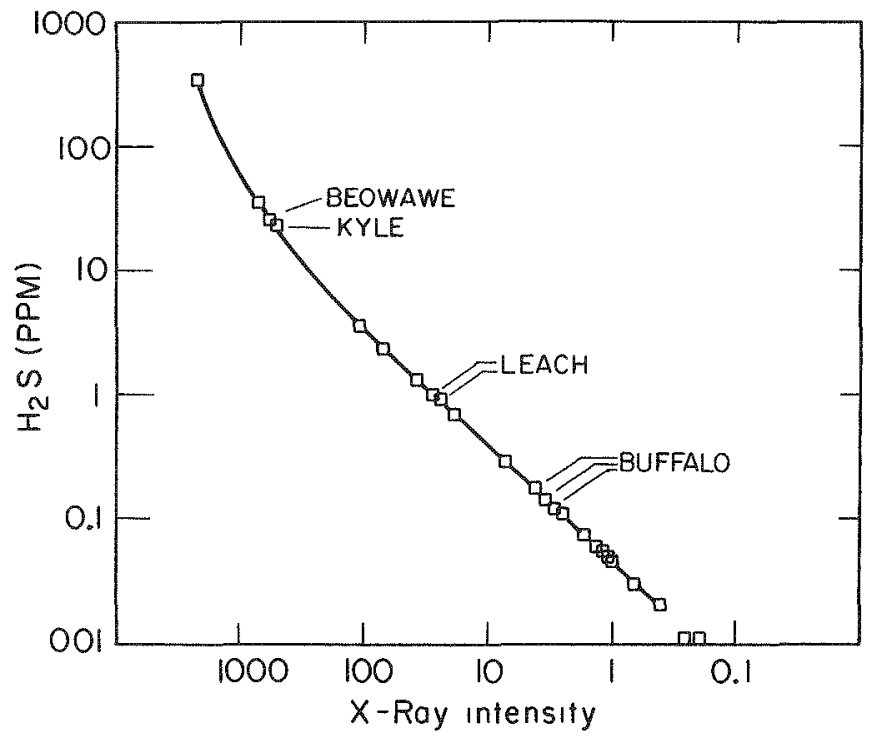

Fig. 2. A calibration curve showing sulfide levels versus observed sulfur soft $X$-ray intensity.

(XBL 747 3624)

\section{FOOTNOTES AND REFERENCES}

1. A. J. Hebert and Kenneth Street, Jr., Anal Chem. 46, 203-207 (1974).

2 H. Bowman, A. Hebert, H. Wollenberg, and F. Asaro, "A Detalled Chemical and Radiometric Study of Geothermal Waters and Associated Rock Formations, with Environmental Implications," presented at the Second International Conference on Nuclear Methods in Environmental Research, University of Missour1, Columbia, Missour1, July 20-31, 1974. 
An instrumentation development program is being undertaken to study combustion phenomena. Most practical combustion systems are both dirty (i.e. contain particles) and turbulent, and most present-day instrumentation in use by combustion researchers is nonspectroscopic. It involves the extraction of combustion products using sampling probes, and hence is inherently incapable of the fine spatial and temporal resolution necessary to study the microscopic details of the processes involved. The program at this moment is to develop $\mathrm{NO}_{\mathrm{x}}$ monitors, based on monitoring the fluorescence excited by a laser.

The development of an $\mathrm{NO}_{2}$ monitor is the first step. The gas mixed in smal1 concentrations in air, would be excited by an $\mathrm{Ar}^{+}$laser. The fluorescence spectra excited from $\mathrm{NO}_{2}$ would be characterized for the available $\mathrm{Ax}^{+}$wavelengths. $\left(\mathrm{NO}_{2}\right.$ fluoresces when excited by light in a wide part of the visible spectrum). Figure 1 shows typical spectra for two laser wavelengths $\lambda_{\mathrm{L}_{1}}$ and $\lambda_{\mathrm{L}_{2}}$. The ratio

$$
r=\frac{\int^{\Delta \lambda} S_{1}(\lambda) d \lambda}{\int^{\Delta \lambda} s_{2}(\lambda) d \lambda}
$$

is characteristic of the species $\mathrm{NO}_{2}$. This test ratio $r$ will be used to determine if the fluorescent species is $\mathrm{NO}_{2}$, thus discriminating against other fluorescent species and particulates.

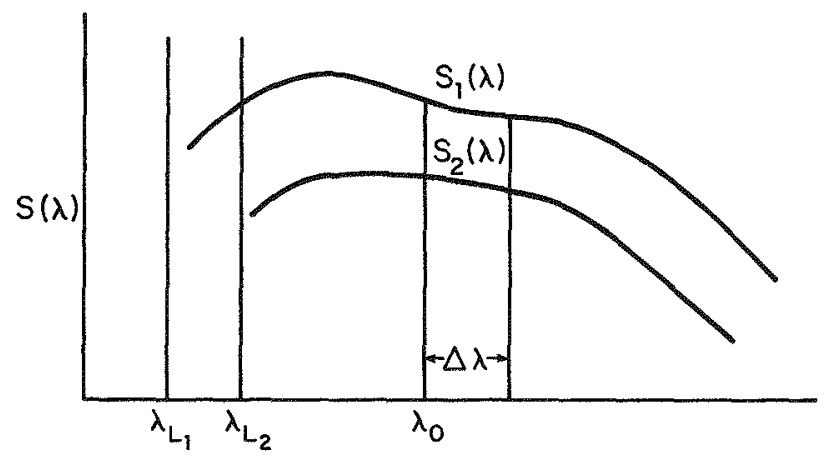

Fig. 1. Typical fluorescence spectra excited by two laser wavelengths $\left(\lambda_{L_{1}}\right.$ and $\left.\lambda_{L_{2}}\right)$. The

ratio of the integrated intensity over bandpass $\Delta \lambda$ provides a characteristics test constant $r$ for $\mathrm{NO}_{2}$.

(XBL 761-2014)
Figure 2 shows the schematic of the $\mathrm{NO}_{2}$ detector under development. Two wavelengths are extracted from the $\mathrm{Ar}^{+}$laser. The plane of polarization of $\lambda_{1}$ is rotated $90^{\circ}$ by use of a quarterwave retardation plate. Both beams then pass through a variable retardation plate whose axis of polarization is controlled by an oscillator. Thus, only one beam passes through at any instant. The beam is focussed by a diffraction-limited lens to a narrow diameter in the measurement region. The monochromator allows fluorescent light of bandwidth $\Delta \lambda$ to fall on the photodetector. The resultant ratio $r$ is tested electronically and, if it is within tolerance 1 imits, one validated measurement is recorded on a computer. The intensity of the fluorescence

signal, $\int^{\Delta \lambda} S_{1}(\lambda) d \lambda$ indicates the number density of the fluorescent species.

The identical method will be employed with NO detection. However, No has selected strong absorption bands in the ultraviolet and therefore new light sources such as a frequency doubled $\mathrm{N}_{2}$ pumped dye laser or atomic lamps must be used.

The achievable spatial resolution is essentially diffraction-1imited. The time resolution will depend on the strength of the fluorescence signal in the absolute (i.e. effective emission crosssection) as we11 as in comparison to the flame background.

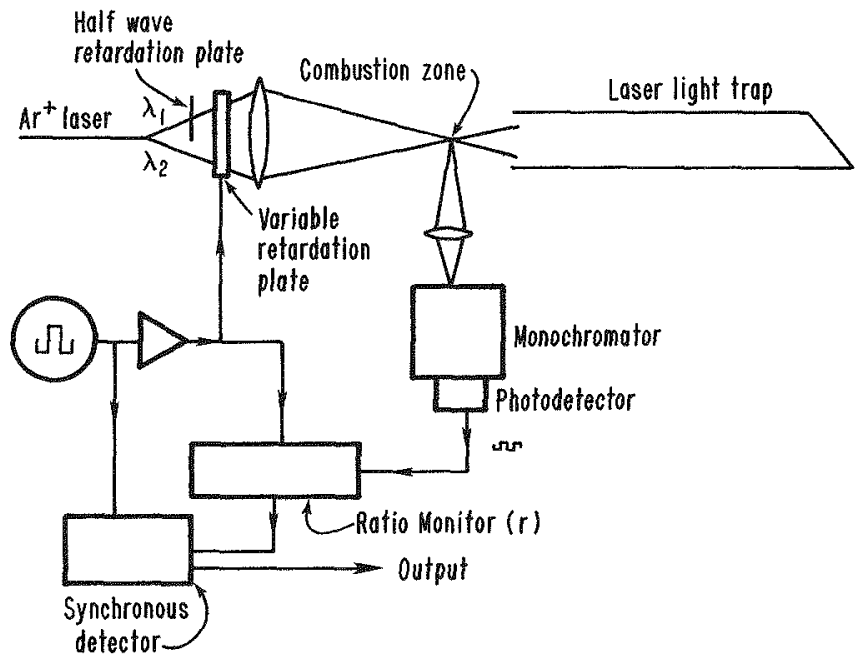

Fig. 2. Schematic diagram showing $\mathrm{NO}_{2}$ detector. (XBL 747-36 


\title{
New Technology and Spin-off Research
}

\author{
INTRODUCTION
}

This section includes reports on work related to new technologies that are not dealt with elsewhere in this report and reports on research that has been made possible by capabilities developed in other division projects but which is not necessarily related to energy or the environment.

New technologies described here concern studies of the feasibility of using intense bursts of high energy electrons for rock excavation, and work directed toward improvement of the Bay Area Rapid Transit(BART) District's automatic train contro1 system. The former brought LBL's experience in particle accelerator design and construction to bear on a problem of great importance in the construction of tunnels for aqueducts and roadways. In the BART project, the Laboratory assisted in the development of train control procedures that enabled the Transit District to initiate revenue service in the San Francisco Bay tunnel.
Sickle cell anemia and luminescence in cadmium sulfide are the subjects of two reports on "spinoff" research. The sickle cell anemia work grew out of an attempt to preserve parts of the photochemical system in chloroplasts with the aim of constructing an in vitro system for the production of hydrogen by solar energy (see Solar Energy Section). In the course of these investigations it was realized that the same strategy applied in a much milder manner might be useful in preventing the sickling of human erythrocytes.

The studies of luminescence in cadmium sulfide were made possible by the development of a powerful laser for use in Raman spectroscopy (see Instrumentation and Measurement section). The results obtained are of interest primarily in basic solid state physics. However an understanding of cadmium sulfide may be of use in the development of photovoltaic cells for the direct conversion. of solar energy to electricity.

\section{INIFNSE SUB-MICROSECOND ELECIRON BURSTS PRODUCE ROCK SHATTERING}

Robert T. Avery, Denis Keefe, Tor L. Brekke and Iain Finnie

Tests have demonstrated that an intense shortduration pulse of energetic $(z 1 \mathrm{MV})$ electrons can produce significant volume removal in a variety of rocks. Some results from these tests, together with discussion of the associated microsecond fracture processes, are given below. The prospects of applying this technique to rapid tunneling through hard rock are also discussed.

Rock samples ranging from very hard to soft were tested, including greenstone, granite, basalt, limestone, sandstone, shale and damp adobe clay. Removal of rock from the face was demonstrated on al1 types. In general, for the same energy imput, softer rock shows greater spall volume than hard rock and wet rock exhibits greater spall volume than dry rock. The spal1 debris is of fine nature, being either sand, dust or small flakes. Spal1 volumes of typically one to almost a hundred cubic centimeters are produced by a single burst of electrons of less than one microsecond duration and having from 3 to 64 kilojoules of energy content. This technique has been labeled "shock spalling." A few of the bombarded rock samples are shown in Figs. 1 and 2 and the results of some of the bombardments are tabulated in Table 1.

Based on the more than one hundred test shots ducted, the following characteristics of the bck spalling mechanism have been demonstrated:

1. It produces spalls on all rock types tested and on clay.

2. There is a threshold energy input below which spalling does not occur. The threshold value is primarily a function of rock type and moisture content.

3. General1y, the results are reproducible, as shown by repeatable front and rear spalls on identical tests.

4. Stronger and tougher rocks generally show less spalling for the same energy input.

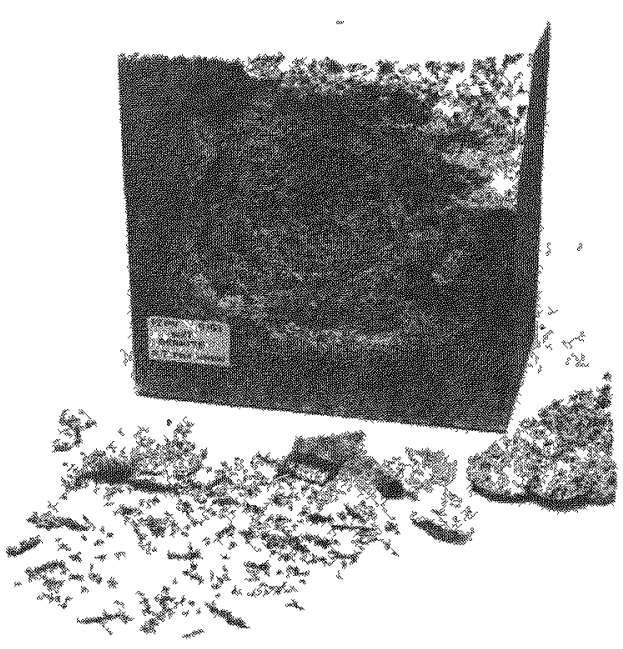

Fig. 1. Wet Sierra granite block $10 \mathrm{~cm}$ thick bombarded in air with single shot $(12.5 \mathrm{MV}$, $64 \mathrm{~kJ}, 160 \mathrm{~ns}$ ) from Hermes II electron accelerator including spa11 debris therefrom. Larger pieces of debris are from upper edge of block and might not have been removed if the block had been larger.

(XBB 741-335) 


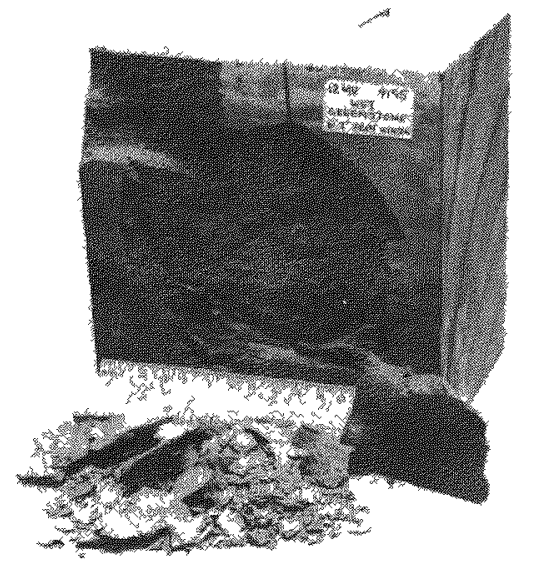

Fig. 2. Wet greenstone block $11 \mathrm{~cm}$ thick bcmbarded in air with single shot (12.5 MV, $64 \mathrm{~kJ}, 160 \mathrm{~ns}$ ) from Hermes II electron acce1erator, including spall debris therefrom. Note crack going through to rear of rock.

(XBB 741-344)

5. Wet rocks generally show more spalling than dry rocks for the same energy input.

6. Wet granite at $0^{\circ} \mathrm{C}, 50^{\circ} \mathrm{C}$, and $75^{\circ} \mathrm{C}$ spalls similarly to wet granite at room temperature.

7. Oven-dry granite spalls the same as roomdry granite.

8. Rocks bombarded in vacuum also spall.

9. The spall size and depth at the front surface are independent of the thickness of the rock sample bombarded (tested only at thicknesses $\geq 2.5$ electron penetration depths) .
10. If the energy fluence of a beam pulse is substantially above threshold for front surface spalling and the rock sample is thin, spalling can also occur at a rear free surface.

11. Successive nearby pulses (but separated in time by many minutes) generally enhance the spalling process.

12. Greater accelerating voltage produces deeper spalls and generally shows more favorable specific energy.

13. Spall debris is small flakes, sand and dust which should facilitate debris removal by hydraulic slurry or pneumatic means.

14. In several cases, substantial cracking occurred in the rock body outside of the spall zone.

The electrons deposit substantially all of their energy content as an impulse of heat within the volume of rock immediately below the rock surface. The restrained thermal expansion produces a compressive stresswave that reflects from the free front surface as a tensile stresswave of short time duration $(\sim 1 \mu \mathrm{s})$. Spalling due to this tensile stresswave appears to be the dominant fracture mechanism. Calculations indicate that the magnitude of the tensile stresswave needed to cause fracture is significantly greater than the static tensile strength of the rock. Considering that cracks can propagate only very short distances during transit of the tensile stresswave, the higher dynamic strength can be explained quantatitively by the requirement that fracture occurs essentially simultaneously at a multitude of nucleation centers across the rock face. For wet rock, there also is evidence that heating of the water in the rock interstices by both direct electron beam heating and by thermal diffusion from the rock volume produces a thermo-hydraulic pressure which contributes to the greater spall volume observed.

Table 1 Single-shot spall data for several tests using Hermes Il Accelerator, January 1974

\begin{tabular}{|c|c|c|c|c|c|c|}
\hline & & $\begin{array}{c}\text { Colorado } \\
\text { Red } \\
\text { Sandstone }\end{array}$ & $\begin{array}{c}\text { White } \\
\text { Limestone } \\
\text { (Marble) }\end{array}$ & $\begin{array}{l}\text { Sierra } \\
\text { Granite } \\
\end{array}$ & $\begin{array}{c}\text { Napa } \\
\text { Basalt }\end{array}$ & Greenstone \\
\hline Compressive strength & $\begin{array}{l}\mathrm{MN} / \mathrm{m}^{2} \\
10^{3} \mathrm{psi}\end{array}$ & $\begin{array}{r}43 \\
6\end{array}$ & $\begin{array}{r}58 \\
8\end{array}$ & $\begin{array}{r}180 \\
26\end{array}$ & $\begin{array}{r}320 \\
46\end{array}$ & $\begin{array}{r}270 \\
40\end{array}$ \\
\hline Young's modulus of elasticity & $\begin{array}{l}\mathrm{GN} / \mathrm{m}^{2} \\
10^{6} \mathrm{ps} 1\end{array}$ & $\begin{array}{c}13 \\
1.9\end{array}$ & $\begin{array}{r}41 \\
6\end{array}$ & $\begin{array}{r}54 \\
8\end{array}$ & $\begin{array}{l}72 \\
10\end{array}$ & $\begin{array}{l}99 \\
14\end{array}$ \\
\hline Shot 1 dentification number & & 9163 & 9165 & 9151 & 9152 & 9155 \\
\hline Mean accelerating voltage & MV & 9 & 9 & 9 & 9 & 9 \\
\hline Standoff distance & $\mathrm{cm}$ & 18 & 18 & 18 & 18 & 18 \\
\hline Total energy deposited & $\mathrm{kJ}$ & 64 & 64 & 64 & 64 & 64 \\
\hline Spall diameter & $\mathrm{cm}$ & 12 & 12 & 13 & 13 & 12 \\
\hline Spall area & $\mathrm{cm}^{2}$ & $\sim 110$ & -110 & -130 & -130 & -110 \\
\hline Spall depth, max. & $\mathrm{cm}$ & 1.5 & 1.1 & 1.1 & 1.0 & 0.7 \\
\hline Volume removed & $\mathrm{cm}^{3}$ & 82 & 57 & 75 & 53 & 51 \\
\hline $\begin{array}{l}\text { Specific energy } \\
\text { (Energy deposited/volume removed) }\end{array}$ & $\mathrm{kJ} / \mathrm{cm}^{3}$ & 0.78 & 1.11 & 0.85 & 1.21 & 1.25 \\
\hline
\end{tabular}


Tunneling, mining and other excavation in ock are promising applications for the shock palling technique, although it is clear that additional research and engineering are needed. The national need to reduce the cost and increase the speed of underground excavation and tunneling is well known. The specific energy levels reported here may be low enough for economic feasibility, but even lower values appear likely. Beam parameters not yet tested may produce more efficient spalling. Lateral compressive stresses due to residual heat during high-repetition-rate bombardment as well as those generally prevailing in in-situ rock may enhance the shock spalling efficiency. Also, residual damage from earlier pulses should enhance spalling during repetitive bombardment. In addition, a variety of strategies for using shock spalling in combination with other methods can be considered, such as cutting a pattern of grooves by shock spalling followed by removal of intermediate material by mechanical means. These improvement factors lead to the expectation of specific energies in the range of 100 to 1,000 joules $/ \mathrm{cm}^{3}$ or perhaps lower.

The prospects appear promising for technical and economic feasibility of a pulsed electron tunnel excavator capable of tumel advance rates approaching 75 meters ( 250 feet) per day through hard rock. This compares to conventional tunneling rates through hard rock of the order of 9 meters ( 30 feet) per 24-hour day under favorable conditions.

\section{References}

1. R. T. Avery, D. Keefe, T. L. Brekke and I. Finnie, "Shattering Rock with Intense Bursts of Energetic Electrons." IEEE Trans, on Nuc1. Sci., Vo1. NS-20, No. 3 (June 1973). (Proceedings of 1973 Particle Accelerator Conference, San Francisco, Calif., March 5-7, 1973.) Also published as preprint report LBL-1391 (1973).

2. R. T. Avery, D. Keefe, T. L. Brekke and I. Finnie, "Use of Intense Sub-Microsecond Electron Bursts to Produce Rock Shattering." Presented at 3rd Int. Congress on Rock Mechanics, Denver, Colorado, Sept. 1-7, 1974. Published in conference proceedings and also published as preprint report LBL-2137 (Jan. 1974).

3. R. T. Avery, "Study of Rock Shattering by Intense Bursts of Energetic Electrons," D. Eng. Thesis, U. of Calif., Berkeley. Also published as report $\mathrm{LBL}-3019$.

\section{STUDIES CONCERNING RAPID TRANSIT ${ }^{*}$}

D. Theodore Scalise, Don M. Evans, Allan A. Arthur, David Bereznai, Martin Graham Raymond Louis, Kenneth G. Wiley

LBL's association with the rapid transit industry began in 1974 when the California Public Utilities Commission and the Bay Area Rapid Transit District (BART) had come to an impasse over certification to begin full operation. The chairman of the State Senate Committee on Public Utilities, Transit and Energy, asked LBL to give technical advice on BART's readiness to begin safe service and also the impact of safety problems on its economic future. A contract with BART itself followed, so that now LBL provides technical help to BART to improve reliability and advice to the Senate Committee with respect to safety provisions.

At the time LBL began work, difficulties concerning the reliability of the automatic train control (ATC) system presented BART with two problems. First, the District needed an interim backup system for ATC that could be implemented rapidly so that Trans-Bay revenue service could be initiated. Second, a permanent improvement to the ATC that would enable the transit system to be operated at its full design capacity was required.

The ATC back-up system used by BART in the eration of its trains divided the track into es separated by stations. The back-up system, lown as the Computer Augmented Block System (CABS), was initially operated in a mode which required a two station separation between trains. However, that much separation made it impossible to operate revenue trains on the key link in the
BART system, the Trans-Bay tumnel. In order to begin Trans-Bay service, BART undertook a modification of CABS so that trains could be operated with a one station separation. LBL participated in an evaluation of the modification and made a number of recommendations which were implemented to improve the safety and integrity of the system.

An important LBL recommendation was to provide Zero Speed Gates in the system. These electronic gates were installed at the exit of each station to stop trains automatically in case of station run-throughs.

After a full-scale test of the system in August, BART initiated Trans-Bay service in September. Operating experience since that time has shown that there have been station runthroughs by trains which could have resulted in a serious safety hazard for passengers of the trains had they not been stopped by the Zero Speed Gates.

The BART Board of Directors formally acknow1edged the important role of the Laboratory in allowing BART to achieve successful Trans-Bay service. On November 14, 1974, LBL was presented a citation by the Board which "does hereby take public cognizance of the inestimable value of the work of the Lawrence Berkeley Laboratory in improving the level of service ... contributing to the state of the art of the entire rapid transit industry ..." 
The CABS ATC back-up system, however, does present some limitation on operating efficiency. Most important1y, CABS requires that trains be operated with six minute headways. This is three times longer than the design headways, consequently the system cannot be operated at full capacity.

Since the beginning of this project LBL has been involved in monitoring work on a permanent back-up system to replace CABS. This back-up system is known as the Sequential Occupancy Release (SOR) system and requires that positive occupancies occur along the route of travel before releasing occupancy indications behind trains.

The basic Iogic of the SOR system was agreed upon before LBL began work. However, a number of design problems remained to be settled. LBL participated in an evaluation of the proposed design of SOR and made a number of reconmendations which were incorporated in the system. The most important of these was that the system should be implemented using digital processors as opposed to a hard-wired logic system. This configuration will make it possible for the system to evolve as new problems are identified.

Currently, LBL engineers and computer scientists are engaged in a program to improve the reliability of BART's trains and control systems. The program includes monitoring the implementation of the SOR back-up for the ATC and a study of other problems, such as worst-case braking rates, that are critical to attaining full-service.

\section{FOOTNOTE}

*Work supported by the State of California Senate Committee on Public Utilities, Transit and Energy and by the San Francisco Bay Area Rapid Transit District.

\title{
PREVENTION OF SICKLING OF HUMAN ERYTHROCYTES BY IMTDOESTERS*
}

\author{
L. Packer and E. N. Bymun
}

Sickle cell anemia is a debilitating and ultimately lethal genetic disorder. The molecular defect in the disease involves the substitution of a molecule of valine for glutamic acid in the sixth position of the $\beta$ chain of hemoglobin. This defect is manifested in a number of abnormal properties in the erythrocytes. Most notably, deoxygenated sickle erythrocytes assume a characteristic "sickle" shape.

Recently, a number of investigations have been directed towards developing a treatment for the sickle cell disease. Several attempts to inhibit sickling by modification of the hemoglobin molecule in vitro have been successful. Nitrogen mustard has been reported to inhibit sickling by a direct effect on hemoglobin gelation. ${ }^{1}$ Cyanate appears to act by modifying hemoglobin oxygen affinity. ${ }^{2}, 3$ clinical trials suggest that cyanate may be therapeutically beneficial in sickle cell disease, but potential side effects exist. It is generally believed that the anti-sickling activity of cyanate resides in its ability to carbamylate the $N$-terminal amino residues of the $\alpha$ chains of hemoglobin.

Experiments conducted by us this past year have suggested the potential usefulness of a new class of compounds as anti-sickling agents. We have demonstrated that the treatment of sickle cells with imidoesters results in the prevention of in vitro sickling. 4-6 The imidoesters under study and their amidination reactions with proteins are illustrated in Fig. 1. Proteins that have been amidinated show minimal alteration of enzyme activity, antigenicity, and physical properties. At slightly alkaline $\mathrm{pH}$, imidoesters react preferentially with $\varepsilon$ amino groups of proteins.

The action of the imidoesters under study is

\section{IMIDOESTERS}

MONOFUNCTIONAL
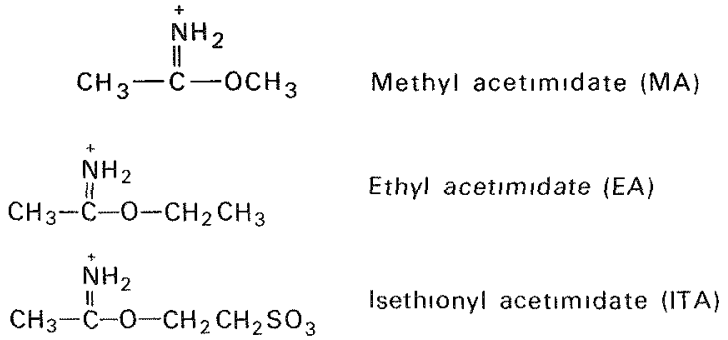

\section{BIFUNCTIONAL}

$$
\begin{aligned}
\stackrel{+}{N} \mathrm{H}_{2} & \stackrel{+}{N} \mathrm{H}_{2} \\
\mathrm{H}_{3} \mathrm{C}-\mathrm{O}-\stackrel{\mathrm{C}}{\mathrm{C}}-\mathrm{R}-\mathrm{C}-\mathrm{O}-\mathrm{CH}_{3} & \\
\mathrm{R} & =-\mathrm{CH}_{2}-\quad \text { Dimethylmalonimidate (DMM) } \\
\mathrm{R} & =-\left(\mathrm{CH}_{2}\right)_{4}-\quad \text { Dimethyladıpımıdate (DMA) } \\
\mathrm{R} & =-\left(\mathrm{CH}_{2}\right)_{6}-\quad \text { Dimethylsuberimidate (DMS) } \\
\mathrm{R} & =-\left(\mathrm{CH}_{2}\right)_{2}-\mathrm{S}-\mathrm{S}-\left(\mathrm{CH}_{2}\right)_{2}
\end{aligned}
$$

Dithı-bis-Dimethylproprioimidate (DMSSP)

\section{AMINIDATION WITH BIFUNCTIONAL REAGENTS}

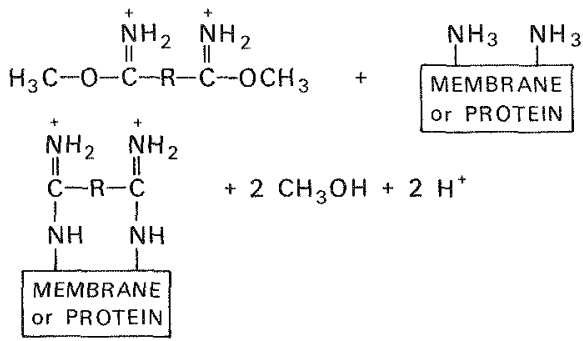

Fig. 1. Structure and reaction of imidoesters being investigated as anti-sickling agents.

(XBL 751-4642) 
shown in Fig. 2. In completely deoxygenated sickle erythrocytes, $50 \%$ inhibition of sickling occurs vith a concentration of the bifunctional imidoesters DMA and DMS of about 500 um for a solution with a $20 \%$ hematocrit. The monofunctional reagent EA is almost as effective. On the other hand, ITA, a monofunctional reagent that does not readily permeate membranes because of its $\mathrm{SO}_{3}^{-}$group, has very 1ittle effect. In other experiments we have found that DMSSP is also an effective anti-sickling agent in the same general concentration range as DMA, DMS, and EA. Furthermore, DMSSP, a cleaveable bifunctional reagent, inhibits sickling even after the di-thio bridge is broken by adding reduced thiols.

The consequences of treating sickle erythrocytes with these reagents is being investigated in terms of a variety of structural and metabolic parameters. For example, our studies with DMA show that the net $\mathrm{K}^{+}$loss from sickle cells incubated under hypoxic conditions, a parameter which has regarded as a quantitative measure of the amount of sickling, is virtually eliminated.

The viscosity of deoxygenated sickle erythrocytes, because of their shape, is greater than norma1 cells. The viscosity of DMA treated and control cells was measured in a cone plate microviscometer at several shear rates. It was found that the viscosity of DMA treated sickle erythrocytes equilibrated with $95 \%$ nitrogen plus $5 \% \mathrm{CO}_{2}$ was in the range of normal cells.

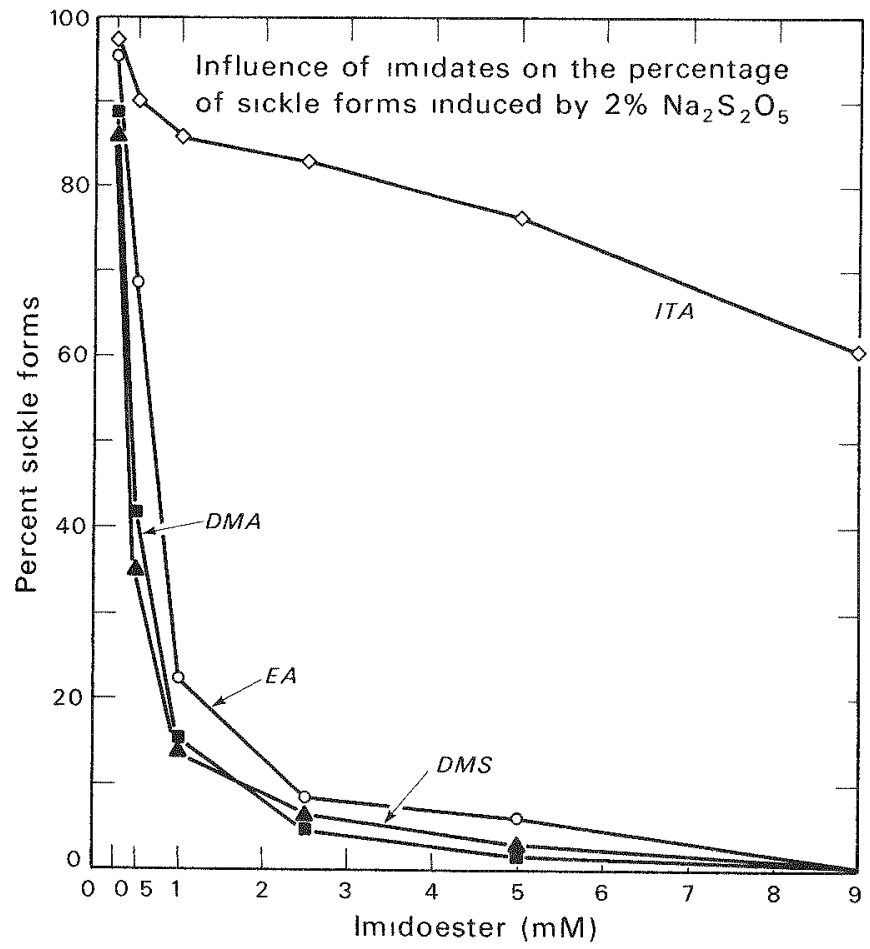

Fig. 2. Prevention of the sickling of human erythrocytes in vitro under completely deoxygenated conditions. In vitro sickling was assessed after deoxygenating the cells with sodium metabisulphide after treatment with $10 \%$ formaldehyde 500 cells were counted to determine the number of fixed sickle cells. Similar results to those shown here have also been obtained in cells that have been equilibrated for one hour with 3\% oxygen. (XBL 751-4644)
Scannung electron microscopy has been used to expose the surface characteristics of sickle erythrocytes. Figure 3 shows the norma1 shape of oxygenated sickle erythrocytes, sickled cells
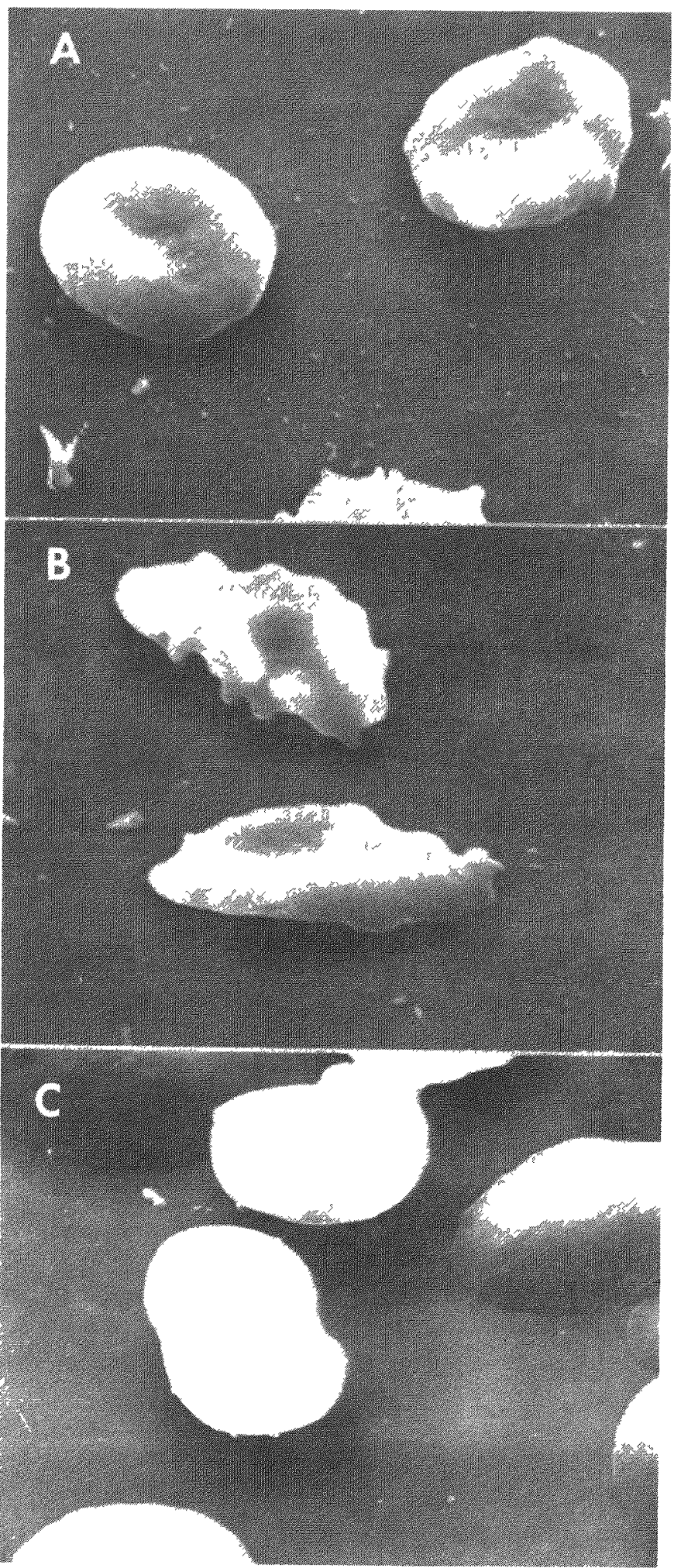

Fig. 3. Scanning electron micrographs of sickle erythrocytes a) oxygenated cells

b) deoxygenated cells, c) deoxygenated cells previously treated with DMA under oxygenated conditions. 
under deoxygenated cells after prior treatment with DMA. These morphological studies taken together with the viscosity data and potassium leakage suggest that the normal rheological properties of sickle erythrocytes are retained after amidination.

Preliminary studies in sickle erythrocytes reveal that $5 \mathrm{mM}$ DMA treatment does not substantially alter glucose consumption, lactage production, i.e., glycolypic enzyne activity. ATP synthesis is also detained.

The whole blook oxygen affinity, expressed as p50 (oxygen tension at which hemoglobin is half saturated) was deternined after equilibration of the samples with gas mixtures containing various concentrations of oxygen (plus $5 \% \mathrm{CO}_{2}$ and the remainder as nitrogen). The hemoglobin oxygen affinity of sickle and normal cells was increased following treatment with DMA.

\section{Amidination reactions by imidoesters as in} this investigation would preferentially react with Lysines of the $\varepsilon$-amino group, but reaction with $\mathrm{N}$-terminal amino groups would also be expected. As a result, imidoesters may act like cyanate to alter hemoglobin oxygen affinity. However, unlike cyanate, inhibition of sickling occurs even under completely deoxygenated conditions indicating that factors other than altered oxygen affinity are also involved.

The mechanism of the inhibition of sickling by imidates is presently unknown. However, the preventing of sickling by the monofunctional EA reagent, suggests that modification of hemoglobin oxygen affinity probably largely accounts for the prevention of sickling. However, a combined effect on both the membrane and hemoglobin may be involved in the action of bifunctional reagents. Thus the degree to which chemical modification and/or crosslinking may account for the effectiveness of bifunctional reagents is unclear.
Although it is premature to anticipate a significant clinical role for imidoesters in the treatment of sickle cell disease, it is of inter est that they act at concentrations several orders of magnitude lower than cyanate. The marked inhibition of sickling and apparent lack of major effects on metabolism suggest that they may be used without seriously disturbing normal red cell physiology. Any therapeutic application of these reagents would likely involve extracorporeal treatment.

Since sickle erythrocytes have a circulating lifetime about half that of normal erythrocytes, it is possible that treatment with the crossliking reagents may be useful in stablizing the functioning 1ifespan of such cells. Stabilization of nomal erythrocytes may also be possible so as to possibly preserve them during in vitro storage. These studies seem sufficiently promising such that further investigation of what might be termed "enzyme engineering" of erythrocytes seems warranted.

\section{Footnotes and References}

"Condensed in part from LBL-3055.

1. E. F. Roth, Jr., G. Neumann, G. Vanderhoff, R. C. Nage1, B. Kaplan, and E. R. Jaffe. Proc. Am. Soc. Hematol., 16th Meeting, p.110 (1973)

2. M. Jensen, F. H. Bunn, G. Halikas, Y. W. Kan, and D. G. Nathan. J. Clin. Invest., 52 , 2542, (1973).

3. P. N. Gillette, C. M. Peterson, Y. S. Lu, and A. Cerami. New Eng. J. Med., 290, 654 (1974).

4. N. I. Krinsky, E. N. Bymun, and L. Packer. Arch. Biochem. Biophys., 160, 350 (1974).

5. B. H. Lubin, V. Pena, W. C. Mentzer, E. N. Bymun, T. B., Bradley, and L. Packer. Proc. Mat. Acad. Sci. USA, 7 (1975) .

6. E. N. Bymun, M. A. Dissertation, University of Ca1ifornia, Berkeley, January (1975).

\section{LUMINESCENCE OF CAS GENERATED BY HIGH INTENSITY EXCITATION BELOW THE BAND GAP*}

A. Mysyrowicz, ${ }^{\dagger}$ A. J. Schmidt, Y. R. Shen P. Robrish, and H. Rosen

In recent years, photoluminescence under highintensity excitation has been investigated in a large number of semiconductors. In particular, the case of CdS has been studied by several research groups using laser excitation with photon energies larger than the band gap.1-6 Several new features in the luminescence spectrum were observed. The observed spectra and their intensity dependence are in good qualitative agreement anong various groups, although the interpretations vary to a considerable extent. Most people, however, believe that the new features in the spectrum are due to a high exciton concentration and the existence of exciton molecules. It is conceivable that by studying the Iuminescence with tunable laser excitations around the exciton lines and the band edge, one might obtain some clarifying information about these new features. We report a preliminary attempt along this line.

The experiments were carried out on pure vaporphase grown CdS single crystal platelets that were inmersed in 1iquid helium below the $\lambda$ point. At all laser excitation frequencies we studied, either below or above the band gap $(2.582 \mathrm{eV})$, with or without linear absorption, the luminescence spectra under sufficiently high-intensity excitations showed features reminiscent of results in previous reports, where the excitation frequenc were always larger than the band gap. As an example, we present in Fig. 1 the intensity-dependent luminescence spectra at two excitation frequencies, one at $2.5542 \mathrm{eV}$ close to the peak of the $A_{I}$ (longitudina1.) excitation, and the other at $2.551 \mathrm{eV}$ 


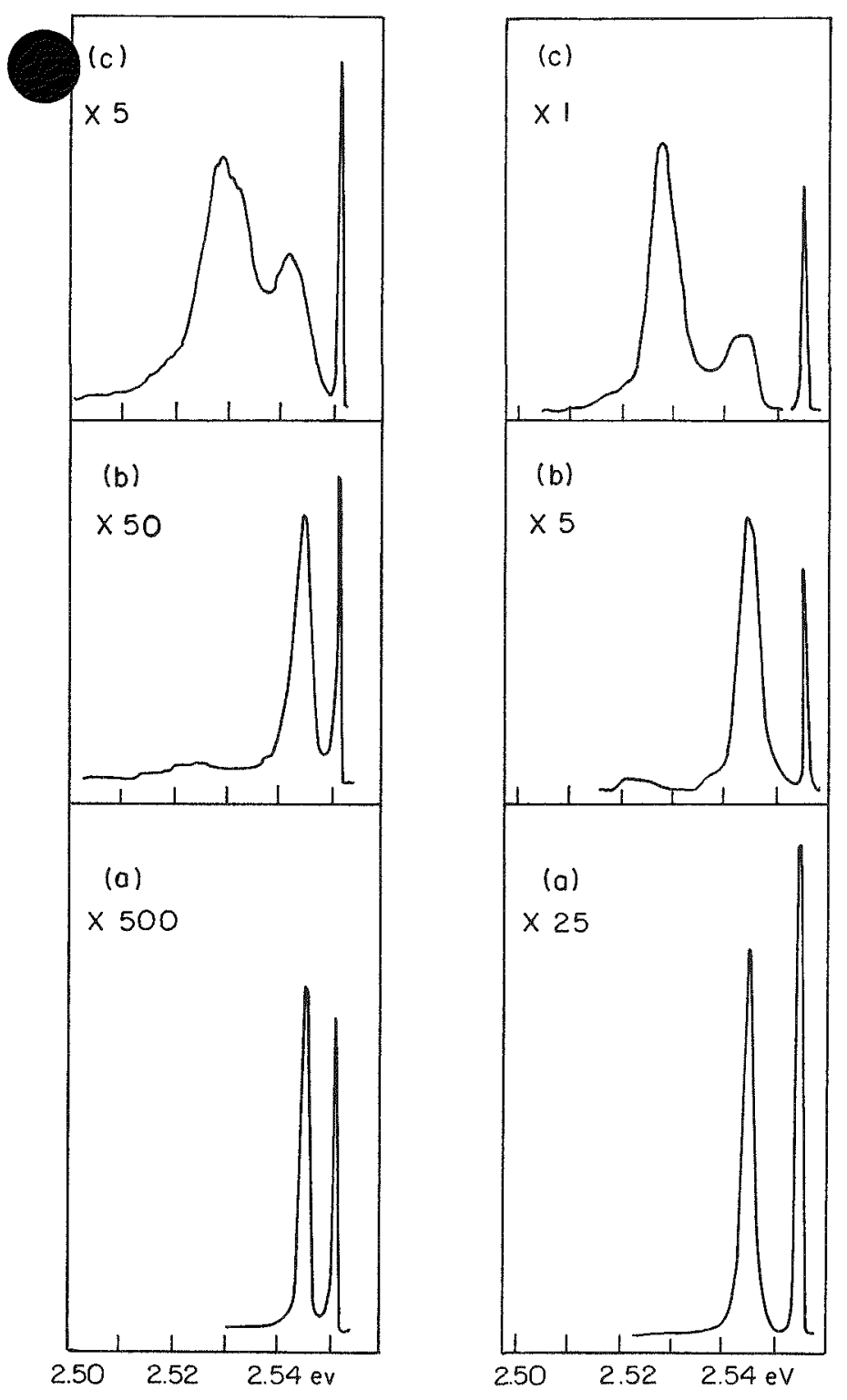

Fig. 1. Intensity dependence of the luminescence of CdS for two selected excitation frequencies; $h \nu=2.5511 \mathrm{eV}($ left $)$ and $h \nu=2.5542 \mathrm{eV}$ (right). The spectra were taken in the backward geometry. The exciting light is polarized I to the c-axis of the sample. The luminescence is predominantly polarized $\perp$ to $\mathrm{c}$. The excitation laser intensities are:

(a) $=0.01 \mathrm{MW} / \mathrm{cm}^{2} ; \quad$ (b) $0.1 \mathrm{MW} / \mathrm{cm}^{2}$;

(c) $=1 \mathrm{MW} / \mathrm{cm}^{2}$. The luminescence spectra are magnified by the amount shown in the figure. The sharp line on the right side of the spectra is due to elastically scattered laser 1ight.

(XBL 7411-7601)

where no linear absorption structure was observed.

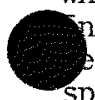
both cases, at the lowest intensity (Fig. 1a), obtained the usual low-excitation luminescence spectrum. At higher excitation intensity (Fig. 1b), a broad line (called M) appeared on the low energy side of the bound exciton 1 ine $\mathrm{I}_{2}$ and started to dominate the spectrum. With even higher excitation intensity (Fig. 1c), another broad line (called P) centered around $2.53 \mathrm{eV}$ appeared and grew nonlinearly with the excitation intensity. It eventually outgrew the $M$ line and its peak shifted slightly to lower energy.

The design of our laser did not allow us to monitor the luminescence intensity under constant laser excitation while the laser was continuously tuned over a large frequency range. We could, however, do so over small frequency intervals. As shown in Fig. 2a, with low excitation intensity, ( $\leq 1 \mu \mathrm{J}$ per pulse), the excitation spectrum of

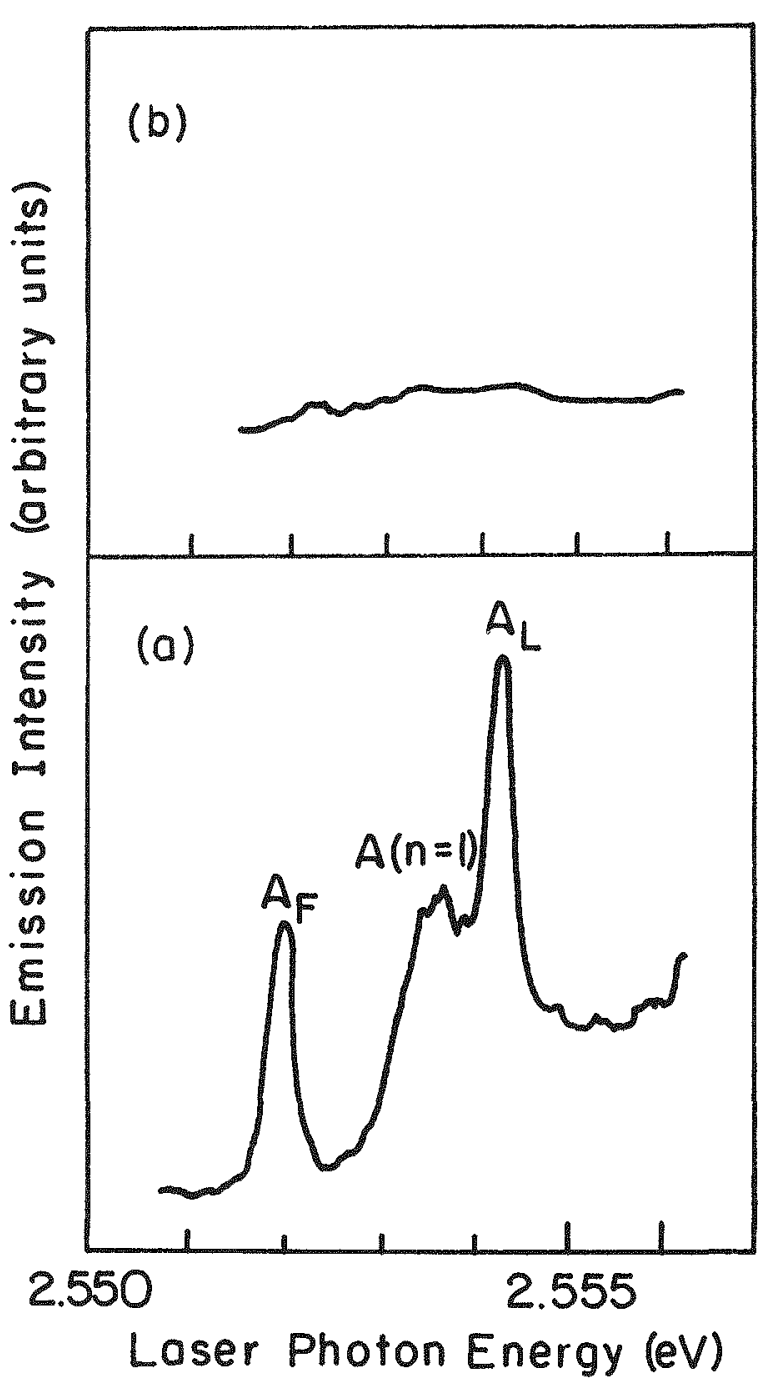

Fig. 2. (a) Excitation spectrum of the $I_{2}$ emission line (backward geometry). The spectrometer bandpass (1.5 MeV) was centered at the peak of the $I_{2}$ line and the excitation frequency was scanned over a region near the A $(n=1)$ exciton line with a laser intensity of $\sim 0.03 \mathrm{MW} / \mathrm{cm}^{2}$. The input polarization was parallel to $c$ and the output polarization perpendicular to $c$.

2(b) Excitation spectrum of the M line taken under similar conditions but with a laser intensity of $1 \mathrm{MW} / \mathrm{cm}^{2}$. Note that the emission intensity in (b) is much larger than in (a).

(XBL 7411-7603) 
the $\mathrm{I}_{2}$ luminescence line shows clear resonant enhancement at the three exciton lines $A_{L}(2.5542$ $\mathrm{eV}), \mathrm{A}(2.5534 \mathrm{eV})$, and $A_{F}(2.5510 \mathrm{eV})$. But with high excitation intensity, the excitation spectrum of the M line showed no sharp structure but only a general decline towards the low frequency side.

We did measure the luminescence spectra of CdS at a constant high-excitation level ( $8.8 \mathrm{\mu J}$ per laser pulse) while varying the laser excitation frequency between 2.5300 and $2.5693 \mathrm{eV}$ in steps of $2.2 \mathrm{MeV}$. Figure 3 summarizes the results for laser polarizations both perpendicular and

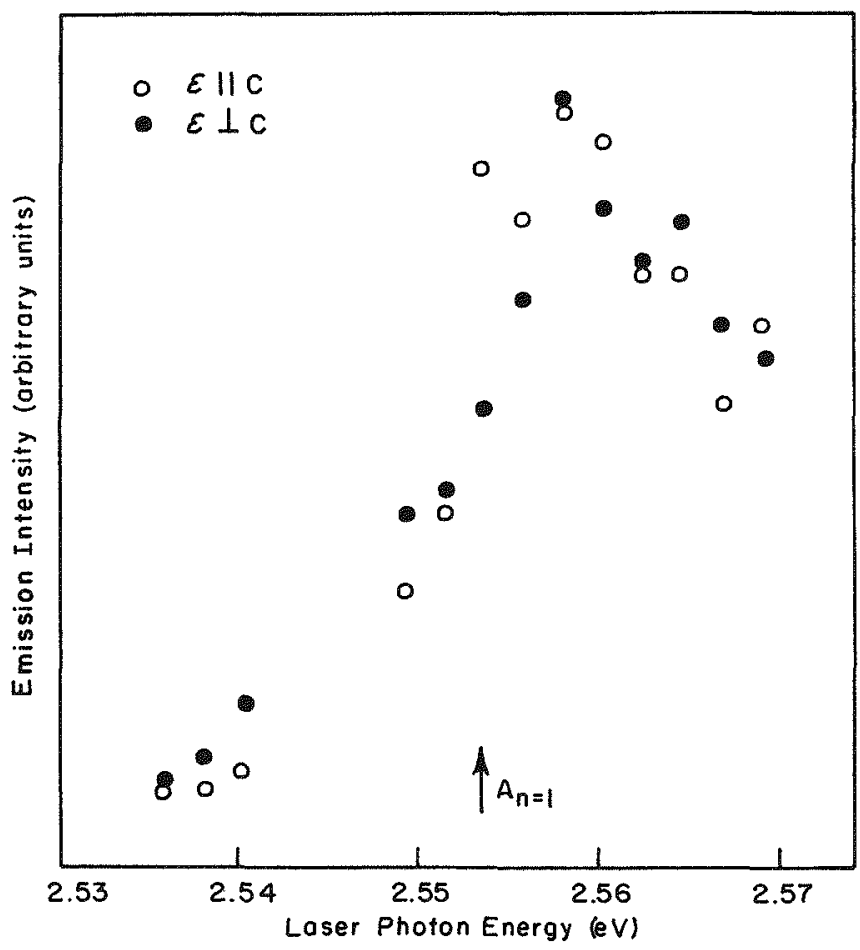

Fig. 3. Peak intensity of M line luminescence as a function of excitation photon energy for input polarization $\vec{\varepsilon} \| c$ and $\vec{\varepsilon} \perp \mathrm{c}$. The data were taken in the forward geometry with a laser power of $0.5 \mathrm{MW} / \mathrm{cm}^{2}$.

(XBL 7411-7602) parallel to the c-axis. The peak intensity of the $M$ line first increases slowly with decreasing excitation frequency, reaches a peak near the A exciton lines, and then drops below the noise level at $\sim 2.535 \mathrm{eV}$. There is no obvious fine structure in these excitation spectra.

Shionoya et al. ${ }^{4}$ have designated the M line as arising from the decay of excitonic molecules. Hanamura ${ }^{7}$ has predicted the direct formation of excitonic molecules via giant two-photon absorption. Gale and Mysyrowicz 8 have observed such a resonant line in the excitation spectrum of exciton-molecular luminescence in CuCl. However, we could not find any resonant enhancement in the excitation spectrum of the M line (see Fig. 3) in the region corresponding to a molecular binding energy given by either Figueira and Mahr, 3 or Shionoya et a1., 4 or to any other reasonable binding energy. This suggests that either the M line is not due to decay of excitonic molecules, or that the corresponding molecular state cannot be reached by direct two-photon absorption.

\section{Footnotes and References}

*Research supported by National Science Foundation, RANN Division and the U.S.E.R.D.A. condensed from LBL-3544.

†n leave from Laboratoire d'Optique Quantique du CNRS, école polytechnique, Palaiseau, France.

1. D. Magde and H. Mahr, Phys. Rev. Letters 24, 890 (1970)

2. D. Magde and H. Mahr, Phys. Rev. B2, 4098 (1970).

3. J. F. Figueira and H. Mahr, Phys. Rev. B7, 4520 (1973).

4. S. Shionoya, H. Saito, E. Hanamura, and 0. Akimoto, Solid State Communications 12, 223 (1973) .

5. H. Saito, S. Shionoya, and E. Hanamura, Solid State Communications 12, 227 (1973).

6. A. F. Dite, V. I. Revenko, V. B. Timofeev, and P. D. Altukhov, JETP Letters 18, 341 (1973).

7. E. Hanamura, Solid State Communications 12 , 951 (1973).

8. G. M. Gale and A. Mysyrowics, Phys. Rev. Letters 32, 727 (1974). 


\title{
Fossil Energy Research and Development
}

\author{
INTRODUCTION
}

The emphasis in our fossil energy work is on the conversion of coal to cleaner and more convenient fuels. This emphasis is derived both from the recognition that coal, by far the Nation's largest fossil fuel resource, must play an increasingly important role in the Nation's energy future, and from the fact that the problem of coal conversion presents a major technical challenge to the scientific and engineering community.

Coal as a fuel suffers from two disadvantages: 1) it is a solid, and 2) it is dirty (i.e., impure). As a solid it requires very sophisticated and expensive combustion apparatus for efficient burning. As a dirty fuel it presents a hazard both to human health and to the environment when it is bumed. For these reasons it is desirable to convert coal to liquid (the most convenient) or gaseous (the cleanest) fuels.

Coal conversion is not a new problem $\cdots$ the production of liquid and gaseous fuels from coal has been often demonstrated in some instances on a commercial basis. However, existing methods are not at present economically competitive. This is largely because of the extreme conditions required for coal conversion in these processes and the in- efficiency of the processes. One way to improve processes for coal conversion is by developing better methods for catalysis of the reactions involed. This problem of catalysis is at the heart of our coal research program.

We are approaching the problem of catalysis on three fronts. First the fundamental mechanisms of catalysis are examined by sophisticated physical methods. Second, improved methods are sought for bringing catalysts into contact with coal. And third, systematic searches are made for better catalysts for coal Iiquefaction and gasification.

Al1 of the Energy and Environment Division's coal conversion work was initiated in July of 1974. For this reason the results that are reported here are preliminaxy and we have emphasized our plans for future work.

A project related to fossil energy in a somewhat more advanced stage is a study of the catalyst/ absorbents for stack gas cleaning. In this project iron oxide has been shown to be potentially useful for a dry process that not only achieves high NO and $\mathrm{SO}_{2}$ removals, but also converts NO to innocuous materials and $\mathrm{SO}_{2}$ to a saleable product.

\section{EVALUATION OF CATALYSTS FOR COAL GASIFICATION}

M. Veraa and A. T. Bell

As presently conceived, the conversion of coal into synthetic natural gas requires three steps: 1) gasification to produce a mixture of $\mathrm{CO}$ and $\mathrm{H}_{2}, 2$ ) water-gas shift to increase the $\mathrm{H}_{2} / \mathrm{CO}$ ratio, and 3) methanation $\left(\mathrm{CO}+3 \mathrm{H}_{2} \rightarrow \mathrm{CH}_{4}\right.$ $+\mathrm{H}_{2} \mathrm{O}$ ) to produce a final product which is essentially methane. The first of these steps is highly endothermic and requires a partial combustion of the coal to provide the necessary energy. In contrast the methanation step is highly exothermic. Clearly, a combination of these two steps into one would be highly desireable in terms of energy and equipment utilization. Themodynamic calculations show that such a process could be achieved if steam and coal were contacted at temperatures below $300^{\circ} \mathrm{C}$. To accomplish this a catalyst is required that would promote the gasification reaction at these temperatures.

The objective of this project is to identify ctical catalysts which can be used to achieve a duction in the gasification temperature without a sacrifice in the rate. To date an apparatus has been designed and constructed to study the rates of gasification of coal samples impregnated with a catalyst. In this apparatus the sample is suspended in a high temperature furnace from the beam of a microbalance. Gasification is achieved by passing a mixture of steam and oxygen or hydrogen over the sample. The composition of the product gases is determined by gas chromatography.

Our current work is focused on the screening of potential catalysts. These materials will include transition and alkalai metals we well as their oxides and sulfides. The catalyst will be introduced into the coal pore structure by standard impregnation techniques from a solvent in which the catalyst precursor (i.e., a salt or complex) is soluble. Coals of different rank will be examined to assess the effects of $\mathrm{C} / \mathrm{H}$ ratio on the rate of gasification.

Once a series of candidate catalysts has been identified attention will be directed to establishing the effects of gasification conditions, namely temperature, pressure, and gas composition. During this phase of the work, attention will also be devoted to identifying the effects that coal pretreatments such as partial oxidation or heating in an inert environment might have on the gasification rates and products. 


\title{
COAL CONVERSION CATALYSIS
}

\author{
Donald W. Blakely and G. A. Somorjai
}

We have begun a study of the catalyzed surface reactions leading to the 1 iquefaction of coal by metal ligand catalysts. A high-ash bonded coal was studied by Auger electron spectroscopy and $x$-ray photoelectron spectroscopy. All the elements present in the bulk coal ( $\mathrm{C}, \mathrm{N}, \mathrm{O}, \mathrm{Al}, \mathrm{Ti}, \mathrm{Si}, \mathrm{S}, \mathrm{Ca}$, Fe) were detectable within the top 10 to $20 \AA$ of the surface, with much higher concentration on fractured surfaces than an polished surfaces. With these techniques the chemical bonding changes during catalysis may be monitored. As a first step we are studying synthetic coals. These are pure, highly aromatic hydrocarbon polymers similar to ordinary plastics. They will be studied for surface crystallinity, electronic conductance and low energy electron damage as well as catalytic depolymerization.

\section{SELECTIVE HYDROGENATION OF COAL: CHEMICAL ENGINEERING ASPECTS}

E. A. Grens, E. E. Petersen, A. T. Be11, T. Vermeulen, K. Conklin, T. Derencsenyi, D. Draeme1, L. Jossens, D. J. Medeiros, and S. Salim

\section{INTRODUCTION}

This project has as its objective the development of methods for conversion of coal into 1iquid "synthetic crude oil" or petrochemical feedstocks, and/or ash- and sulfur-free solid fuel, at lower temperatures and pressures than are required for existing methods. The general approach we have taken is that of selective hydrogenation or hydrogenolysis of the coal, especially through use of homogeneous catalysis. Since coal is composed to a large extent of fused-ring hydroaromatic structures, connected by chemical bridges such as $-\mathrm{CH}_{2-},-\mathrm{O}_{-},-\mathrm{S}-,-\mathrm{C}_{-} \mathrm{O}-$, and $-\mathrm{NH}-$, these bridges appear susceptible to selective scission by processes of the type considered here.

Progress in the past year, the first year for the project, has occurred in four interrelated aspects of the project: 1) studies of the action of organic hydrogen donor solvents on coal, 2) investigation of the effect of reaction and extraction on the physical structure of coal, 3) catalytic hydrogenation of organic structures resembling those in coal; and 4) examination of the interaction of coal with molten salt solutions. All our tests have been with a western sub-bituminous coal from the Roland seam of the Wyodak mine, Wyoming.

EFFECT OF HYDROGEN-DONOR SOLVENTS ON COAL (Grens, Draeme1)

Experiments have been initiated to clarify the nature and extent of the effect of hydrogendonor solvents, such as tetralin, on coal under well-controlled conditions. They consist in subjecting the coal to the solvent in question in a continuous Soxhlet type extraction unit at controlled temperature and pressure for extended periods. The yield and characteristics of the extract, including molecular-weight distribution, are determined as functions of reaction conditions. The effects on solid residue are examined in related work.

A high-pressure Soxhlet-type extraction unit has been constructed capable of operating at pressures of from 0 to $\sim 700$ psia (47 atm) and at temperatures of from $\sim 70^{\circ}$ to $700^{\circ} \mathrm{F}\left(21^{\circ}\right.$ to $370^{\circ} \mathrm{C}$ ). The unit operates at solvent boiling temerature, with temperature automatically controlled through pressure control. Temperature deviations are generally less than $\pm 1^{\circ} \mathrm{C}$. The system is capable of continuous automatic operation for long periods of time, i.e., hundreds of hours.

Several experiments on Roland seam coal have been conducted with tetralin and decalin as solvents, as well as with hexane as a control (non-hydrogen donor) solvent. These extractions have been conducted at $150^{\circ}, 200^{\circ}$ and $250^{\circ} \mathrm{C}$ for 2 and 4 hours. Additional runs have been made for 60 hours and "to completion" (as determined by evaporation of extraction solvent) or $\sim 200$ hours; see Figs. 1 and 2.

The extracted coal samples will be characterized by Medieros and Petersen with BET surface area, mercury intrusion porosimetry and He displacement density. The extract materials are being studied with NMR, UN and IR spectroscopy. Their molecular weight distributions are being determined by vapor pressure osmometry as well as gel permeation chromatography. Preliminary results

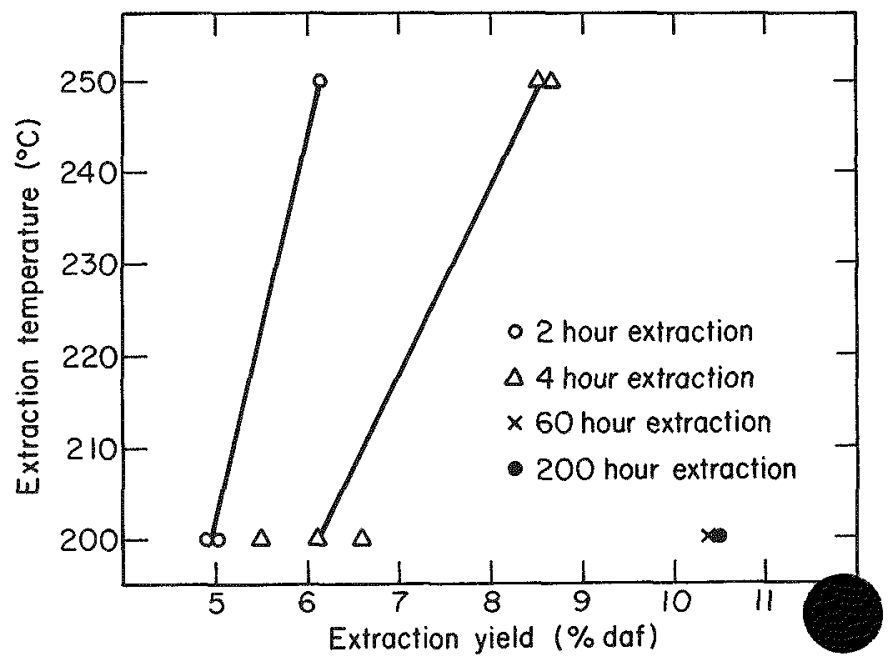

Fig. 1. Extraction temperature vs. extraction yield for -28 mesh Roland Seam coal extracted with tetralin.

(XBL, 761-2015) 


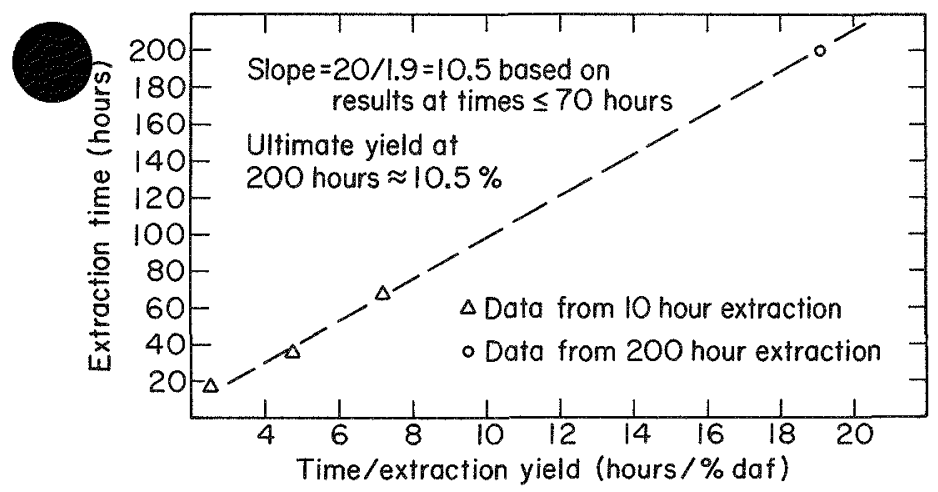

Fig. 2. Time of extraction vs, ratio of time to yield for -28 mesh Roland Seam coal extracted with tetralin at $200^{\circ} \mathrm{C}$.

(XBL 761-2016)

indicate average molecular weights in the range of several hundred with a distribution including species exceeding 2000 in molecular weight.

Extract yields have been studied as a function of time of extraction by drawing samples from the Soxhlet cup. Rate/time relationships have confirmed that accurate extrapolation to the utlimate yield (which genera11y occurs after 200-400 hours of extraction) can be achieved from data taken up to $\sim 60$ hours of extraction. This result must be verified for each coal-solvent pair, but it is expected to hold for most common hydrogen donnor solvents. For example, for tetralin at $200^{\circ} \mathrm{C}$, the data obtained up to 70 hours of extraction predicts an ultimate yield of $10.5 \%$ (d.a.f.) of extract, the same as the actual yield obtained after complete (200-hour) extraction.

\section{INTERNAL SURFACE PROPERTIES OF COAL (Petersen, Medeiros)}

The changing properties of coal during processing are important indicators of the types of treating action, and also need to be known in connection with utilization of the solid residue. As the internal structure of the coal changes, so do transport properties that affect the design of process equipment. The objective of this study is to analyze the residues from extraction and reactions for variations in the following physical properties: 1) internal surface area of the coal, 2) porosity, and 3) pore distribution within the coa1. The internal surface area of the coal is being determined by means of adsorption of inert molecules at both low and elevated temperatures by the well known BET method. Porosity and poresize distribution measurements are being made in a mercury porosimeter and with a helium density apparatus.

The recent literature indicates a controversy on the sorption of inert molecules, such as nitrogen and argon at 1iquid-nitrogen temperatures on coal, since nitrogen is used extensively for surace-area measurements on other porous materials. any theories have been suggested to explain why adsorption of nitrogen at liquid nitrogen temperatures yields a lower surface area than adsorption with carbon dioxide at dry-ice temperatures, but none of these theories has yet been fully explored. New leads on this problem are being developed.
To facilitate the experimental objectives of this project, a four-sample BET apparatus has been constructed of stainless steel. The unit is capable of measuring surface areas as $10 \mathrm{w}$ as $2 \mathrm{~m}^{2} / \mathrm{g}$ (coal generally has a surface area in the range of $80-250 \mathrm{~m}^{2} / \mathrm{g}$ ). Calibration against standard materials has been completed, and studies of gas adsorption on raw coal have begun. The results obtained to date confirm the controversial information that nitrogen shows a lower surface area than carbon dioxide, at the respective temperatures indicated above.

We have also begun pore-structure and density measurements using a 5,000 psi porosimeter. The results are consistent with a model of coal containing very fine micropore structure, with the majority of pores in the $5 \AA$ diameter range. A1though the $5,000 \mathrm{psi}$ porosimeter can only measure pores greater than $350 \AA$ in diameter, density measurements can extend this range. However, in order to measure pores in the range of $5 \AA$, a higherpressure porosimeter operating at $100,000 \mathrm{psi}$ is required.

\section{CATALYTIC HYDROGENATION AND HYDROGENOLYSIS OF} ORGANIC STRUCTURES RESEMBLING THOSE FOUND IN COAL (Be11, Conklin)

Work has been initiated on the development and evaluation of catalysts for the hydrogenation, hydrogenolysis, desulfurization, and denitrogenation of coal. For this work, model compounds are used that contain structures representative of those found in coal. By using compounds having well-defined structures rather than coal, it is possible to identify the reaction products and thereby detemine the reaction pathway. This approach makes it possible to classify catalysts according to their activity for particular functions and will assist in the rational development of new catalyst systems.

A high pressure stirred autoclave has been assembled and instrumented. Reactions are carried out by charging the autoclave with a solvent such as benzene or tetralin in which the reactant is dissolved. The catalyst (homogeneous or heterogeneous) is dissolved or suspended in the solution. After the reaction temperature and pressure have been achieved, samples of the liquid are withdrawn periodically and analyzed by gas chromatography.

The initial studies are directed at the hydrogenolysis of aromatic hydrocarbons structures. For this work, bibenzyl, diphenyl methane, and diphenyl are used as the substrate or reactant. The emphasis is on finding catalysts capable of breaking the aliphatic bridge between the phenyl groups at moderate temperatures. This step simulates the depolymerization of coal which consists primarily of fused aromatic rings held together by aliphatic bridges.

The hydrogenation and decyclization of anthracene and phenanthracene is also being studied. These molecules are representative of the aromatic fractions of coal. Ring opening processes are extremely important to the reduction in size of the aromatic clusters and to the formation of benzene and toluene which are highly desired constituents of 1iquid fuels. 
Very shortly we will begin work on the removal of sulfur and nitrogen. Here again model compounds containing these atoms in representative structures will be used to simulate coal. As a part of this work, the effects of sulfur and nitrogen as catalyst poisons will be examined.

INTERACTION OF COAL WITH MOLTEN-SALT SOLUTIONS (Vermeulen, Derencsenyi, Jossens)

We have begun testing a group of inorganic compounds that can be liquefied by small admixtures of water, and are likely to be effective as dispersants or solvents for coal, as well as solvents for homogeneous catalysts. Such salt-and-water mixtures are quite unlike dilute aqueous solutions, and instead resemble the higher-temperature liquid form (or "melt") of the pure compound. These melts usually have higher solvency and higher reactivity toward organic compounds than water. Easily liquefied inorganic salts that are of potential interest include chlorides such as $\mathrm{ZnCI}_{2}$ (melting point $\left.310^{\circ} \mathrm{C}\right), \mathrm{FeCl}_{3}\left(280^{\circ} \mathrm{C}\right), \mathrm{SnCl}_{2}\left(250^{\circ} \mathrm{C}\right)$, and $\mathrm{Cu}_{2} \mathrm{Cl}_{2}\left(422^{\circ} \mathrm{C}\right)$; phosphates and phosphites such as $\mathrm{KH}_{2} \mathrm{PO}_{4}\left(256^{\circ} \mathrm{C}\right)$ and $\mathrm{CaHPO}_{3}\left(\mathrm{Ca} .250^{\circ} \mathrm{C}\right)$; sulfates such as $\mathrm{KHSO}_{4}\left(210^{\circ} \mathrm{C}\right)$ and $\mathrm{K}_{2} \mathrm{~S}_{2} \mathrm{O}_{7}\left(300^{\circ} \mathrm{C}\right)$; and hydroxides including $\mathrm{NaOH}\left(320^{\circ} \mathrm{C}\right)$ and $\mathrm{KOH}\left(380^{\circ} \mathrm{C}\right)$. Numerous runs have been made with zinc chloride melts containing chemical additives, and exploratory work has been started with sodium hydroxide.

The tests with zinc chloride have been conducted at $200^{\circ} \mathrm{C}$, under approximately $0.8 \mathrm{~atm}$ of hydrogen and $0.2 \mathrm{~atm}$ of water vapor, with undried coal from Wyodak Corporation's Roland seam preground to 20 to 40 mesh. Microscopic examination has shown that the coal breaks up (within one hour, for mild agitation) to a state in which the coarsest particles are below 100 mesh, and a substantial part of the coal has been dispersed to sub-micron size. Dispersion in zinc chloride is more rapid and more complete than in ferric chloride, and has been shown to occur as well. under nitrogen as under hydrogen.

An economical method is being sought for disengaging the dispersed coal from the $\mathrm{ZnCl}_{2}$ melt. Dissolution of excess anhydrous KCI into the melt, which is known to cause hydrocarbon separation at $400^{\circ} \mathrm{C}, 1$ did not produce any phase separation at $200^{\circ} \mathrm{C}$. Cooled melts were therefore decomposed by pouring them in water initially at $30^{\circ} \mathrm{C}$, in the proportions of 3 volumes of water per volume of melt. The residual carbonaceous material, a brownblack solid that was recovered by filtration, had the texture of an agglomerated powder containing small proportions of grit. Analysis of this residue by $x$-ray fluorescence showed no reduction in sulfur from the feed level of $0.9 \%$, and no reduction in any ash component except calcium. This indicates that more severe treating conditions or more powerful catalysts are required.
The residues, termed "melt-treated coal", showed substantial increases in their content of benzene-extractible material, as determined in a Soxhlet extractor at atmospheric pressure. The material extracted, ranging from 0.5 to $7 \%$ of the feed, was a light-yellow colored waxy solid, melting near $70 \mathrm{C}$. The mass spectrum was dominated by a peak at a composition of $\mathrm{C}_{26} \mathrm{H}_{52} \mathrm{O}_{2}$, which corresponds to an ester or keto-ether structure akin to carnauba wax. Table 1 gives comparative results for different melt treatments, and shows that elemental iodine, a known catalyst for coal conversion, 2 gave the largest yield of waxy extract. It appears that 1ittle hydrogen is consumed in freeing this hydrogen-rich waxy extract from the coal, and that the remainder of the coal has a hydrogen-to-carbon atomic ratio of only about 0.8 . The waxy extract has a higher molecular weight than any fraction yet extracted with organic solvents in the runs by Draemel and Grens.

Table 1. Yield of benzene-extractable fraction from melt-treated coals

$\begin{array}{ll}\text { Untreated coal } & 0.5 \% \\ \mathrm{ZrCl}_{2} \text {-treated, without additive } & 2.3 \% \\ \text { with } 5 \% \mathrm{MgCl}_{2} & 3.5 \% \\ \text { with } 5 \% \mathrm{KI} & 3.4 \% \\ \text { with } 1 \% \mathrm{I}_{2}, 0.6 \% \mathrm{KI} & 7.0 \%\end{array}$

The melt-treated coals are to be examined for porosity and surface area by Medeiros and Petersen, and probably will be subjected also to scanning electron microscope studies. Catalysts found to be effective in model-compound studies, in the tests by Conklin and Bell, will be incorporated into zinc chloride and other melts, in future runs with coal. Also, in forthcoming tests of melt treatment, the temperature range will be widened to include $250^{\circ} \mathrm{C}$, and the total-pressure range to include 10 atmospheres.

\section{REFERENCES}

1. W. P. Scarrah, and L. Berg, paper presented at AIChE National Meeting, Salt Lake City, August 1974.

2. R. T. Eddinger, and L. D. Friedman, Fuel 47, 320 (1968). 


\title{
SYNTHESIS OF HYDROCARBONS FROM CO-H $\mathrm{H}_{2}$ MIXTURES PRODUCED BY COAL GASIFICATION
}

\author{
W. Bollinger, J. Ekerdt, and A. T. Bell
}

The gasification of coal with steam produces a low Btu gas consisting mainly of $\mathrm{CO}$ and $\mathrm{H}_{2}$. High $\mathrm{Btu}\left(\sim 900 \mathrm{Btu} / \mathrm{ft}^{3}\right)$ pipeline gas is produced by converting the $\mathrm{CO}-\mathrm{H}_{2}$ mixture to methane via the reaction

$$
\mathrm{CO}+3 \mathrm{H}_{2} \rightarrow \mathrm{CH}_{4}+\mathrm{H}_{2} \mathrm{O}
$$

As an alternative the $\mathrm{CO}-\mathrm{H}_{2}$ mixture can be used to synthesize methanol or a variety of aliphatic hydrocarbons. The latter process is known as FischerTropsch synthesis. With the decreasing availability of low molecular weight liquid and gaseous fuels from petroleum, their synthesis from coalderived products will become increasingly important.

The aim of the present work is to study the catalysis of hydrocarbon synthesis. The two major processes under consideration are methanation and Fischer-Tropsch synthesis. The major goals of this program are to characterize the catalyst properties required for high activity and selectivity with respect to a given product. This objective is pursued through physical characterization of the catalyst, identification of the structure and quantity of adsorbed species, and measurements of reaction kinetics. Studies of catalyst deactivation and poisoning are also performed with the objective of defining the mechanisms by which these processes proceed. The results of this work will be used to guide the development of more active and stable catalyst systems.

Work on the synthesis of methane from carbon monoxide and hydrogen has already started. It is being done in a specially designed apparatus that allows infrared spectra of the adsorbed species present on the catalyst surface to be recorded under reaction conditions. Concurrent measurements are being obtained of the reaction kinetics. In order to relate spectral band intensities to surface coverage, the degree of chemisorption of reactants and products is being measured gravimetrically in an apparatus constructed especially for this prupose. Experiments have been initiated using a ruthenium catalyst. This material has been selected because of its high activity and specificity for CO methanation and because it will serve as the starting point for the study of a series of ruthenium alloy catalysts.

Future studies will deal with the synthesis of hydrocarbons that have molecular weights higher than that of $\mathrm{CH}_{4}$ (Fischer-Tropsch synthesis). This work will be carried out with iron and iron alloy catalysts.

REDUCTION AND REMOVAL OF $\mathrm{SO}_{2}$ AND NOX FROM SIMULATED FLUE GAS WITH USE OF IRON OXIDE AS CATALYST/ABSORBENT*

David T. Clay ${ }^{\dagger}$ and Scott Lynn

Sulfur dioxide and nitric oxide are major air pollutants in the U. S. Fossil-fueled power plants are primary sources of both. Wet processes for $\mathrm{SO}_{2}$ removal are currently being developed commercially, but to date no process for simultaneous $\mathrm{SO}_{2}$ and NO removal has passed the laboratory scale. A dry removal process would be preferred, since this would avoid flue-gas reheating, problems of contacting the gas with a liquid or slurry, and crystallization phenomena usually encountered in flue-gas scrubbing. The objective of our study was to develop a dry process that not only achieves high $\mathrm{NO}$ and $\mathrm{SO}_{2}$ removals, but also converts NO to innocuous materials and $\mathrm{SO}_{2}$ to a saleable product and in addition, is potentially economical to operate.

The flowsheet for the process under development is shown in Fig. 1. Iron oxide, supported on alumina, acts as a catalyst for the reduction of both $\mathrm{SO}_{2}$ and $\mathrm{NO}$ by $\mathrm{CO}$ and $\mathrm{H}_{2}$. Simultaneously, it acts as an absorbent for sulfur compounds, conrerting them to FeS. The catalyst/absorbent is in
e form of fine peilets $(0.05-0.10 \mathrm{~mm})$ and conacts the gas as a falling bed in co-current flow at $370-540^{\circ} \mathrm{C}$. The solid is regenerated with air at $675^{\circ} \mathrm{C}$ to produce a rich stream of $\mathrm{SO}_{2}$, suitable for conversion to $\mathrm{H}_{2} \mathrm{SO}_{4}$ or elemental sulfur. $\mathrm{Fe}_{2} \mathrm{O}_{3}$ is reformed in the particles.
Experiments in fixed-bed reactors using mixtures of helium and one or more reactant gases were run to demonstrate the basic process chemistry, to estimate rates for preliminary design purposes, and to look for interfering reactions. The overal1 reactions 1 isted in Table 1 were shown to proceed rapidly at $370^{\circ}-540^{\circ} \mathrm{C}$ in fixed-bed reactors using supported iron oxide pellets $3.2 \mathrm{~mm} \times 3.2 \mathrm{~mm}$ or particles $0.25-0.50 \mathrm{~mm}$ diameter. $\mathrm{SO}_{2}$ was reduced and absorbed as FeS by both $\mathrm{CO}$ and $\mathrm{H}_{2}$. NO was reduced to a mixture of $\mathrm{N}_{2}$ and $\mathrm{NH}_{3}$ by either $\mathrm{H}_{2}$ or $\mathrm{CO}+\mathrm{H}_{2} \mathrm{O}$. Stable intermediates in the removal of $\mathrm{SO}_{2}, \mathrm{H}_{2} \mathrm{~S}$ and $\mathrm{COS}$ were found to react directly with $\mathrm{FeO}$ to form $\mathrm{FeS} . \mathrm{O}_{2}$ was also reduced but can lead to poisoning of the catalyst under some conditions of temperature and feed stream composition. Based on breakthrough curves for a bed of particles at $540^{\circ} \mathrm{C}$, greater than $90 \%$ removal of $\mathrm{SO}_{2}, \mathrm{NO}$, and $\mathrm{O}_{2}$ occurred in residence times in the reaction zone of the order of $0.01 \mathrm{sec}$. Intraparticle diffusion was the rate-limiting step in both removal and regeneration reactions.

Oxidation of the sulfided catalyst/absorbent with air at $680^{\circ} \mathrm{C}$ produced a rich stream of $\mathrm{SO}_{2}$ and regenerated $\mathrm{Fe}_{2} \mathrm{O}_{3}$. After three successive cycles the solid showed no Indication of loss of activity. The catalyst/absorbent remained reactive only when the inlet gas stream was net reducing. Formation of 


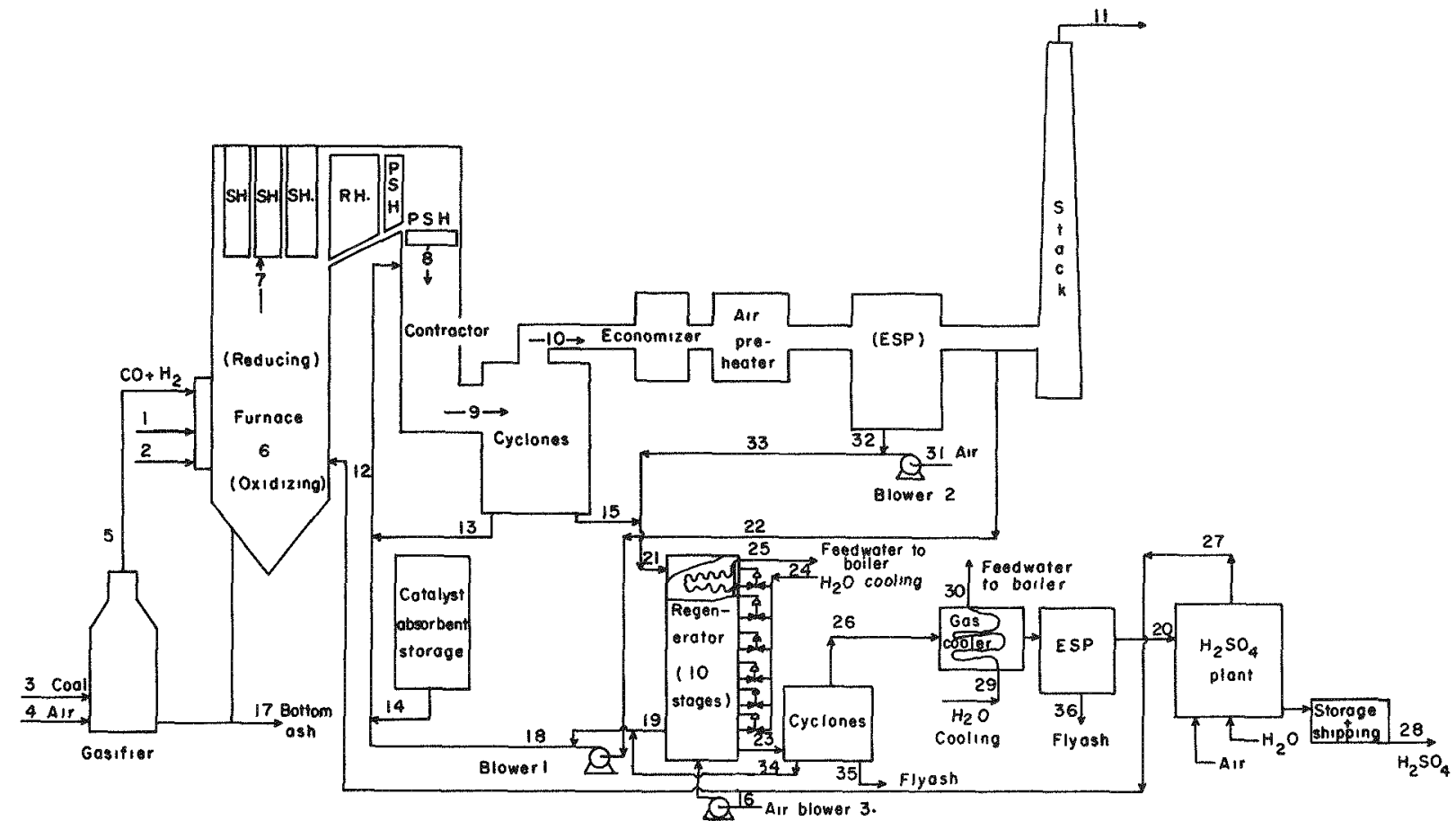

Fig. 1. Conceptual design of $\mathrm{NO} / \mathrm{SO}_{2}$ removal process.

(XBL 746-3399)

Table 1. Overall chemcal reactions for $\mathrm{NO} / \mathrm{SO}_{2}$ removal process.

\section{Absorption/Reduction:}

$$
\begin{aligned}
& 2 \mathrm{NO}+2 \mathrm{CO} \rightarrow \mathrm{N}_{2}+2 \mathrm{CO}_{2} \\
& 2 \mathrm{NO}+2 \mathrm{H}_{2} \rightarrow \mathrm{N}_{2}+2 \mathrm{H}_{2} \mathrm{O} \\
& 2 \mathrm{NO}+3 \mathrm{H}_{2} \mathrm{O}+5 \mathrm{CO} \rightarrow 2 \mathrm{NH}_{3}+5 \mathrm{CO}_{2} \\
& \mathrm{FeO}+\mathrm{SO}_{2}+3 \mathrm{CO} \rightarrow \mathrm{FeS}+3 \mathrm{CO}_{2} \\
& \mathrm{FeO}+\mathrm{SO}_{2}+3 \mathrm{H}_{2} \rightarrow \mathrm{FeS}+3 \mathrm{H}_{2} \mathrm{O} \\
& \mathrm{FeO}+\mathrm{H}_{2} \mathrm{~S} \rightarrow \mathrm{FeS}+\mathrm{H}_{2} \mathrm{O} \\
& \mathrm{FeO}+\mathrm{COS} \rightarrow \mathrm{FeS}+\mathrm{CO}_{2} \\
& \mathrm{FeS}+2 \mathrm{O}_{2} \rightarrow \mathrm{FeSO} \\
& \mathrm{Fe}_{2} \mathrm{O}_{3}+\mathrm{CO} \rightarrow 2 \mathrm{FeO}+\mathrm{CO}_{2} \\
& \mathrm{Fe}_{2} \mathrm{O}_{3}+\mathrm{H}_{2}+2 \mathrm{FeO}+\mathrm{H}_{2} \mathrm{O}
\end{aligned}
$$

Regeneration:

$$
\begin{aligned}
& 2 \mathrm{FeS}+7 / 2 \mathrm{O}_{2} \rightarrow \mathrm{Fe}_{2} \mathrm{O}_{3}+2 \mathrm{SO}_{2} \\
& 2 \mathrm{FeSO}_{4}+\mathrm{Fe}_{2} \mathrm{O}_{3}+2 \mathrm{SO}_{2}+1 / 2 \mathrm{O}_{2} \\
& 2 \mathrm{FeO}+1 / 2 \mathrm{O}_{2} \rightarrow \mathrm{Fe}_{2} \mathrm{O}_{3}
\end{aligned}
$$

$\mathrm{FeSO}_{4}$ from $\mathrm{FeS}$ by reaction with $\mathrm{O}_{2}$ caused poisoning. An excess of $\mathrm{CO}$ and/or $\mathrm{H}_{2}$ minimized $\mathrm{FeSO}_{4}$ formation.

Rate data from the fixed-bed reactor were used to estimate the catalyst flow and particle size necessary to treat the flue gas from a 1000-MW power plant in a dispersed-phase, co-current contactor. The resulting design called for a flow of $0.185 \mathrm{~kg}$ catalyst $/ \mathrm{m}^{3}$ flue gas, a 1 -second residence time, and particles averaging $0.075 \mathrm{~mm}$. The total flow and the particle characteristics are similar to those in fluid-bed catalytic cracking. The regenerator requires a 5-minute hold-up time at about $675^{\circ} \mathrm{C}$ with this size particle.

An economic evaluation of the process based on a tentative plant design indicates an investment cost of about $\$ 19 / \mathrm{KW}$ and an operating cost of about $0.9 \mathrm{mills} / \mathrm{KW}$-hr for a $1000-\mathrm{MW}$ power plant. The investment includes the cost of a conventional sulfuric acid plant, but no credit was taken for $\mathrm{H}_{2} \mathrm{SO}_{4}$. These costs are about half those listed for commercially available recovery processes for $\mathrm{SO}_{2}$ control only 1 and are appreciably lower than the costs for throwaway processes. These cost advantages are inherent to a dry recovery process if sufficient catalyst reactivity and life-time can be demonstrated.

\section{Footnotes and References}

* Condensed from LBL-3059.

${ }^{\dagger}$ Present address: Weyerhouser Corporation, Tongview, Washington 98622

1. F. T. Princiotta, and W. H. Ponder, "Current Status of $\mathrm{SO}_{2}$ Control Technology", presented at the LBL Seminar "Sulfur, Energy, and Environment", April 4, 1974. 


\title{
Geothermal Energy and the Geosciences
}

\author{
INTRODUCTION
}

The Energy and Environment Division's program in geothermal energy and the geosciences is directed toward two goals. These are, first, the development of an important new energy source, and second, the development of fundamental information in the geosciences to serve a broad range of energy needs.

There are three main elements in the program. These are geothermal resource assessment, binary cycle geothermal powerplant development, and supporting scientific and engineering studies. Resource assessment is the largest program element. At present, efforts are focused primarily on exploration at sites in northern Nevada, and involve geological, geophysica1, and geochemical measurements, with the objectives being the evaluation of the specific sites and the development of more powerful methods for exploration.

Binary geomethermal powerplant development efforts are directed toward the use of high-temperature, low-salinity geomthermal brines. Pro- cesses are being examined in which the heat from the brine is exchanged with a secondary fluid that is the working fluid for a turbine generator. Work has included both the analysis of various fluids and cycles and the design of a pilot plant.

The supporting scientific and engineering studies are fairly wide-ranging. They include geochemical and geophysical studies, investigations of thermodynamic and chemical properties of brines, and research on geologic materials. These studies contribute both to the geomthermal energy program and to the development of basic information in the geosciences.

An additional supporting project, the National Geothermal Information Resource, is charged with assembling data on references on geothermal energy. By the publication of manuals and the operation of a computer data base, this project will provide information to all parties interested in geothermal energy.

\section{GEOTHERMAL ENERGY RESOURCE ASSESSMENT}

\author{
H.A. Wollenberg, F. Asaro, H. Bowman, T. McEvilly, \\ $F$. Morrison and $P$. Witherspoon
}

\section{INTRODUCTION}

A principal interest of the LBL Geothermal Program lies in the development and evaluation of methods to locate and produce geothermal energy from moderate temperature high quality brines from 1iquid-dominated systems. Brines of this type range in temperature from 150 to $210^{\circ} \mathrm{C}$ and contain less than 5000 ppm of total disolved solids. They are particularly well suited for the development of electric power through binary cycle systems, because their relatively high heat content, and low salinity, minimize scaling and corrosion problems. Such low salinity brines are expected to furnish the bulk of electrical and non-electrical geothermal energy during the next few decades. trating active fault zones in areas of moderate to Moderate temperature low salinity brines are characteristically associated with deeply penetrating active fault zones in areas of moderate to high heat flow. One such area in the vicinity of Battle Mountain in north central Nevada, has the highest average heat flow in the continental United States. Accordingly, we selected four sites in this area for geothermal energy resource assessment. y are Whirlwind Valley east of Battle Mountain, falo Valley southwest of Battle Mountain, Grass lley south of Winnemucca, and Buena Vista Valley south of Winnemucca. These sites, shown on the 10cation map (Fig. 1) are all essentially on Federal land, and each contains an active hot spring system (Beowawe Hot Springs in Whirlwind Valley, Buffalo

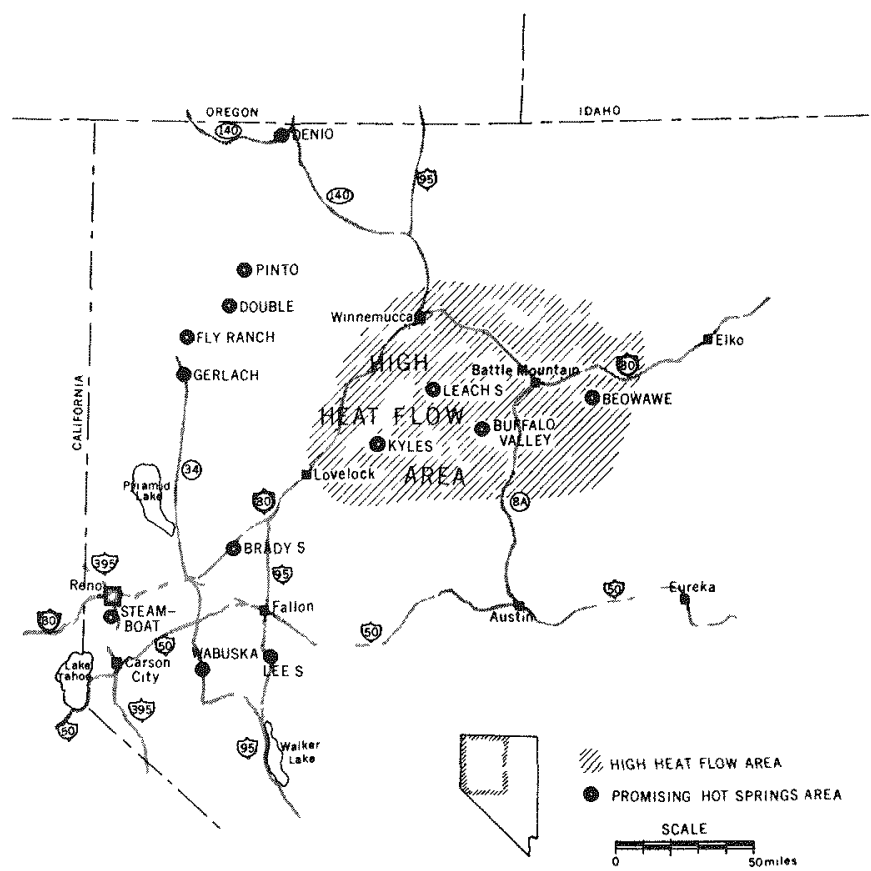

Hot Springs in Northwestern Nevada

Fig. 1. Location map, northwestern Nevada, showing prominent thermal spring areas within and outside of the Battle Mountain high heat flow region.

(XBL $735-676)$ 


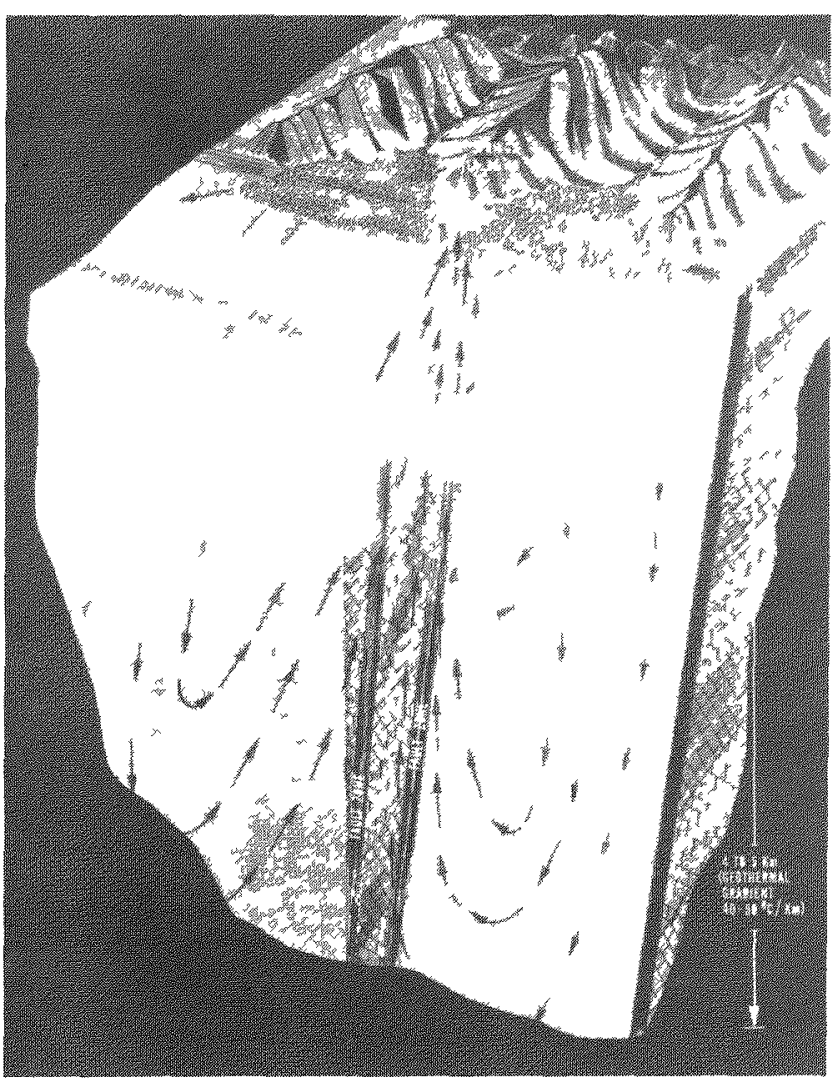

Fig. 2. Schematic cutaway diagram of a geothermal system within a permeable fault zone. Meteoric water enters the fault zone where it intersects near-surface aquifers. Some of the water percolates downward to regions where temperatures reach 150 to $200^{\circ} \mathrm{C}$, is heated and rises on the upward limb of a convection cel1. Hot springs occur where the cell intersects the surface. (CBB 743-1509)

Valley Hot Springs, Leach Hot Springs in Grass Valley, and Kyle Hot Springs in Buena Vista Valley). Temperatures at depth in some hot springs in the area are believed to attain 150 to $170^{\circ} \mathrm{C}$, and contain less than 5000 ppm disolved solids.

A comprehensive systematic approach is required to evaluate the energy potential of a given geothermal resource site. The LBL approach consists of three stages:

1. A reconnaissance phase, in which geologic, geophysical, and geochemical techniques are used to locate and delineate a reservoir, and to specify the location of shallow heat flow holes.

2. An evaluation program in which relatively shallow $(100$ - 200 meter $)$ heat flow holes are drilled. These holes are used to measure conductive heat flow and to define the location and depth of intermediate $(1 / 2$ to $1-1 / 2 \mathrm{~km})$ calibration test holes. Concurrent hydrogeologic model studies furnish the basis for reservoir evaluation as data on heat flow from the shallow holes and temperature profiles, rock permeabilities, and fluid flow from the intermediate calibration test holes are obtained.

3. The drilling of one or more $(1-1 / 2$ to 3 $\mathrm{km}$ ) test wells for reservoir capacity studies.
These wolls would al so furnish the fluxd for a heat exchanger test facılity of a binary system. Surface and subsurface techniques to monitor the configur tion of geothermal reservoirs are tested at existing resource sites, along with downhole methods to evaluate the energy potential of the reservoir.

We report here on the results of work accomplished in 1974 and draw observations and conclusions regarding the geothemal energy potentials of the sites and the effectiveness of the techniques being used.

\section{GENERAL SETTTING}

The Battle Mountaln high heat flow area in north-central Nevada is within the geologica1 Basin and Range province. This province consists of a series of north-south trending ranges separated by broad open valleys partially filled with volcanic detritus and with alluvium from the adjacent ranges. It is believed that the province originated through crustal distention that led to the extensive down-faulting of the valleys and relative uplift of adjacent blocks to form the mountain ranges. Many of the steeply dipping faults are still active, as manifested by numerous recent fault scarps flanking the range fronts, a locus of continuing seismic activity.

Active hot spring areas and potential geothermal resource sites in the Basin and Range province are in almost all cases associated with the intersection of steeply dipping faults. The fault zones furnish permeable pathways for downward percolating meteoric water so that it can reach sufficient depth (4 to $5 \mathrm{~km}$ ) in a region of high geothermal gradient $\left(40\right.$ to $\left.60^{\circ} \mathrm{C} \mathrm{km}\right)$ to be heated to more than $150^{\circ} \mathrm{C}$. The water then rises at the intersection of major fault zones where permeability is high. The subsurface reservolrs of heated water may be located in the fractured rock of the fault zones or $1 \mathrm{n}$ adjacent relatıvely pormeable beds of Tert]ary sedments and Quaternary valley flll alluvium.

\section{TECINIQULS FOR RESERVOIR IDENTII ICATION AND LOCATION}

Field studies must be directed towards eluci dation of the geologic structure and identification and location of the postulated geothermal reservoir. This can be done through geological mapping, geophysical methods, and geochemistry, as discussed below.

\section{Geologic Mapping}

Because much of the area is in valle, fill alluvium, reconnaissance and detall mapping relies heavily on aerial photography. High altıtude $(65,000 \mathrm{ft})$ flights by NASA U-2 aircraft provide regional coverage of high resolution black and white photographs taken at low sun angles, which enhance fault-related features on the desert floor. Low altitude $(6000 \mathrm{ft})$ color photography at higher angles shows details associated with faulting on the immediate site areas. Geologic mans of Buffalo, Grass, and Buena Vista Valleys (Figs. 3 and 7 are examples) were produced prumarily from the interpretation of such aerial photogrpahy, substantiated 
by detailed mapping on the ground.

Airborne infrared imagery obtained by NASA in predawn and mid-day hours indicated the known hot spring areas, as well as a hitherto unknown warm spring area in the west portion of Buffalo Valley playa, and a possible warm spot near the mouth of Sheep Ranch Canyon near Leach Hot Springs. ${ }^{2}$

This geological information provides guidance for orienting geophysical traverses and geochemical samp1ing, and an understanding of the general structural setting necessary for the interpretation of geophysical results.

\section{Geophysics}

The exploration and assessment of geothermal reservoirs is essentially a new technology with little in the way of previous case histories or experience. A number of geophysical methods were therefore tried at the four Nevada sites in order to evaluate their effectiveness.

Recomnaissance techniques are the most critical part of the delineation program because a typical geothermal site comprises an area of several hundred square kilometers in which targets for detailed study have to be selected quickly and efficiently.

Geophysical efforts have concentrated on methods to measure the electrical resistivity and seismicity of an area. Electrical resistivity is important because the resistivity of an electrolyte in the pores of a rock decreases with increase in temperature, and thus the reservoir is often of lower resistance that the surrounding cold rock. Seismicity is important in determining the location of active faults that are believed to control fluid flow in geothermal areas. Seismic velocity ratios may also indicate anomalously warm zones at depth.

Auxiliary geophysical studies such as self potential and gravity have also been undertaken. These methods help interpret the geological structure of an area and may prove to be useful for direct reservoir detection.

DC Resistivity. Electrical resistivity may be determined by measuring the fields produced either by a controlled current source or by distant natural sources. The most common of the controlled current techniques, the resistivity method, is to inject pulsed dc current into the ground between two electrodes and to measure the voltage difference produced between two similar distant electrodes. A reconnaissance version of this method called bipole-dipole consists of injection of a large current with electrodes up to $2 \mathrm{~km}$ apart (the bipole), and measurement of resulting voltages with a roving receiver array using electrodes 100 meters apart (the dipole). This method suffers from an inherent

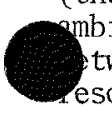
biguity that makes it difficult to distinguish tween shallow and deep inhomogeneities. Better esolution can be achieved with the dipole-dipole array. Here the electrode pairs have equal spacing and are arranged colinearly; the separation between voltage receiver and current transmitter dipoles is an integer multiple of the dipole length.
We have developed a computer program for generating resistivity pseudo sections from dipoledipole data and it is in routine use. A catalog of all the models used in interpretations is now being compiled.

Natural Electromagnetic Fields. Using natural electromagnetic or telluric fields has an obvious advantage over dc resistivity methods because a current source is not required and a broad spectrum of energy is available. The depth of exploration can then be selected without the need for the large arrays required in dc resistivity methods.

A telluric technique using natural low frequency earth currents has been developed that is particularly well suited for reconnaissance surveys. A leap-frogging array of three colinear electrodes spaced $500 \mathrm{~m}$ apart is used to determine the ratio of the electric field across the leading electrode pair to the following electrode pair. This ratio is proportional to the ratio of the ground resistivity beneath the electrode pairs. Successive ratios are referenced to the base, or starting, electrode pair so that a profile of relative resistivity is produced.

The voltages of the leading electrode and the lagging electrode with respect to the center electrode are fed, respectively, to the $x$ and $y$ inputs of a small battery operated $x-y$ recorder. For a signal of given frequency the ratio is easily taken as the slope of the resulting $x-y$ plot.

When the ground becomes highly anisotropic it can be shown theoretically that the electric field ratio becomes dependent on the polarization of the magnetic field inducing the earth currents, especially if the direction of the profile is not in the maximum earth current direction. Further, it is found that this ratio is a function of frequency so that if more than one frequency is present the $x-y$ plot becomes a Lissajou-1ike figure for which a slope cannot easily be estimated.

The field procedure, then, is to take data in profile lines that are approximately parallel to the maximum earth current direction as determined from an initial study using an orthogonal electrode array. Narrow band filtering is used to elininate a multiple frequency effect. The dominant frequency is determined from a chart recording of the telluric field as a function of time, and an appropriate band pass is selected about this center frequency. This passband is then used until the data quality deteriorates, at which time the frequency content is again measured. Several $x-y$ plots are taken for each location so that a mean slope and standard deviation can be calculated. The ratios obtained with these techniques are generally good to $5 \%$.

The method described here requires very simple portable equipment and is operated by only two men. Because it can be used to replace bipole-dipole surveys, which require heavy high power generators, electrode emplacement, and a minimum of four men, the telluric method is a significant advance in geothermal exploration. 
The magnetotelluric method, using the earth's natural electromagnetic fields, provides more quantitative data; here the apparent resistivity of the ground is calculated from measurement of the surface impedance, $\mathrm{E} / \mathrm{H}$, of the incident electromagnetic fields. This method is preferable to that using natural low frequency currents because measurements can be made at a convenient grid of points over the area of interest rather than being restricted to a profile, and because absolute values of resistivity are obtained rather than relative variations. Furthermore, the variation of impedance with frequency can quantitatively reveal the variation of resistivity with depth in the geologic section. We have been using a Josephsoneffect magnetometer for magnetotelluric surveys. This new field sensor provides sensitivities an order of magnitude better than existing induction coil sensors and is easier to deploy in the field.

Magnetotellurics promises to be the best recomaissance method for geothermal delineation. With improved data acquisition and processing capability it should provide greater detail and conductivity resolution than other reconnaissance methods.

Self Potential. Theoretical analysis and some limited field data suggest that geothermal activity might produce associated dc fields. The sources for these fields are either the motion of conducting fluids in a porous medium or the result of themoelectric effects. Due to the great variation in fluid flow properties of rocks it is difficult to make quantitative estimates of the streaming potentials in given geologic situations. However, anomalies of 50 to 100 millivolts are often observed in areas of active flow, especially along faults. Thus the flow regime in a geothermal area may have a good self-potential expression.

Thermoelectric potentials for a large hot buried sphere representative of a geothermal reservoir have been calculated for the site delineation study in Nevada, 3 and they show that negative values of self potential as great as $60 \mathrm{mV}$ could occur. Direct detection of a hot volume at depth might therefore be possible.

A preliminary analysis of two self-potential surveys in Nevada ${ }^{3}$ reveal that:

(1) Distinct self-potential anomalies are associated with the geothermal activity. Strong anomalies, believed to be associated with upwelling thermal fluids along a prominent fault passing through the hot springs, were discovered.

(2) Electrode response to changes in soil chemistry and moisture content appears to be the major source of irreproducibility and background noise in self-potential surveys.

(3) Long wavelength anomalies associated with deep seated thermoelectric sources would almost certainly be concealed by the noise sources described in (2) and by the survey procedure, which, in traversing large distances using short electrode spreads, accumulates significant error.

A complete report of the work thus far accomplished is being prepared for release in 1975 .

Gravity. Gravity data were taken on a profile in Buffalo Valley and on the electrical lines in Grass Valley. The required level survey has not yet been carried out, and field data are not yet reduced to the standard Bouguer anomaly form. A rough data reduction is in progress, and may be useful in resolving the complex faulting near Leach Hot Springs.

Seismic Studies. There is evidence that geothermal reservoirs might be detected and located through the presence of microseismicity. Preliminary surveys of September and Decenber 1973 at Buffalo Valley and Whirlwind Valley indicate the existence of microseismicity near Buffalo Valley, but showed that a one-month recording period in each area of interest is required to locate a reasonable number of hypocenters. Consequently, a reconnaissance project in the Battle Mountain area was conducted in 1974 using eight portable smokedpaper MEQ-800 seismographs at each of the four sites. 5 The field technique was to record in a valley with a very wide station coverage, process the data as quickly as possible, then modify the network to concentrate on any clusters of microearthquakes.

Hypocenter location computation was complicated by lack of velocity information in the region and by severe lateral variations in velocity due to the thick valley fill, which thins to zero at the valley edge. Computations were not of sufficient precision for detailed mapping of fault surface geometry because of limited timing resolution.

A 12-station radio linked network has been field tested and is now being used for detailed studies of the seismic activity, ground noise, and variations in velocities and attenuation characteristics in the Battle Mountain area.

Studies of spatial variations in seismic ground noise in several frequency bands in the 1 to $30 \mathrm{~Hz}$ range are being pursued to test the feasibility of such a technique in geothermal resource delineation. In addition, the effect of geothermal zones on propagation of teleseismic body- and surface-waves is being investigated.

\section{Geochemical Studies}

Nuclear analytical techniques currently in use at LBL provide quantitative measurements of elemental abundances in water, rock, and soil samples from geothermal areas. A number of potentially diagnostic elements have been determined which heretofore have not been used in geothermal survey applications.

The major, trace, and radioactive element abundances including non-condensible gas content of hot and cold spring waters from geothermal resource areas in north-central Nevada are being studied. Results of these studies will serve as a baseline for evaluation of the envirommental impact of geothermal resource development.

Analysis of Spring Waters and Associated Rock Units. Water analysis techniques include neutron activation, soft $x$-ray, 6 and gamma ray spectrometric analysis. These methods are capable of detecting and quantifying over 50 elements in 
sample. About 15 elements were usually detected the hot spring waters. Preliminary results rom initial chemical analyses of both hot and cold water systems and associated rocks and spring deposits from four geothermal areas in Nevada suggest that this type of data may lead to indicators of:

(1) The temperatures and rock types within the geothermal system. system.

(2) The size and depth of the convecting

$\operatorname{mix}$.

(3) The extent to which hot and cold waters

(4) The amounts of valuable elements and compounds in these waters.

(5) The extent to which noxious and haz ardous materials are released into the environment.
Natural Radioactivity in Geothermal Waters. We began studying the radioactivity content of the hot spring areas in northern Nevada early in our search for geothemal sites. We felt that a knowledge of the distribution and abundance of radioelements would be useful both as a means of elucidating the underground plumbing of a hot spring and as a factor in evaluating the envirommental impact of the development of geothermal resources.

Gamma radioactivity was measured with a portable $3 \times 3$-in. NaI(Tl) scintillation detector coupled to a count-rate meter. Field radioactivities were measured over hot pools, sinter $\left(\mathrm{SiO}_{2}-\right.$ rich), and tufa ( $\mathrm{CaCO}_{3}$-rich) deposits, and away from the spring areas to obtain background values. Samples of spring-deposit tufa, sinter, springwall muck, and water were collected at all sites, and on return to the laboratory, were analyzed

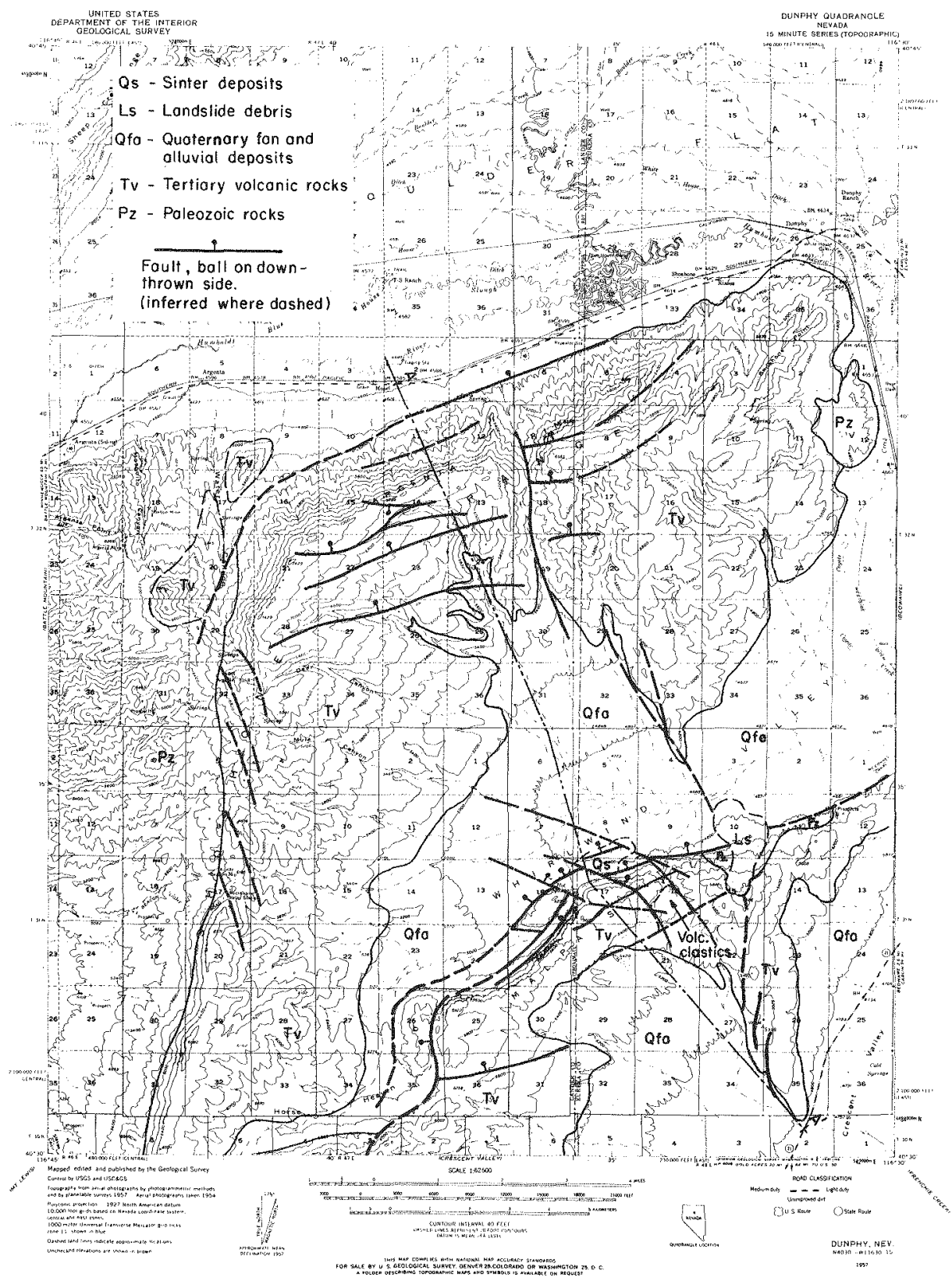

Fig. 3. Generalized geologic map of Whirlwind Valley area.

(XBB 737-4663) 
for $238 \mathrm{U}, 232 \mathrm{Th}$, their daughter products, and $40 \mathrm{~K}$ by gamma-ray spectrometry. Field and laboratory instrumentation and procedures are described in greater detail elsewhere.8 reached.

The following general conclusions have been

(1) Low-flowing $\mathrm{CaCO}_{3}$-dominated spring systems are the most radioactive.

(2) Radium preferentialiy associates with $\mathrm{CaCO}_{3}$ in the Nevada hot-spring deposits.

(3) Where siliceous and calcareous materials are mixed in a hot-spring deposit, the calcareous material has the highest radioactivity.

(4) Radon alpha-track detectors, presently used to delineate concealed uranium deposits, are also sensitive to radon emanation levels associated with radioactive geothermal systems.

Tentatively, it may be concluded that waters in some of the $\mathrm{CaCO}_{3}$-dominated hot-spring systems deposit $226 \mathrm{Ra}$ near the surface of $10 \mathrm{w}$-flowing springs. Most of the $222 \mathrm{Rn}$ observed in these waters is probably derived from decay of $226 \mathrm{Ra}$ deposited on the spring walls.

\section{SITE EVALUATTONS}

A description of work accomplished in 1974 at the four selected sites follows. The status of each site is reviewed, and work planned for 1975 is briefly discussed.

Whirlwind Valley (Beowawe) Site Description and Geology.

The Beowawe geothermal area, approximately $30 \mathrm{~km}$ east of Battle Mountain, consists of active hot springs and blowing hot-water wells at the south margin of Whirlwind Valley, on the lower slopes of the steep Malpais Escarpment. The geology of the area is described by oesterling 9 and is illustrated in Fig. 3. Tertiary volcanic rocks, predominantly andesitic but capped by basalt, have been tilted and fractured along the Argenta Rim, bordering the Humboldt River Va1ley, and along the Malpais Escarpment, prominent topographic features trending ENE-WSW. The area of active springs occupies a broad siliceous sinter apron, approximately $2 \mathrm{~km}$ west of the intersection of the Malpais and a nearly N-S trending zone of en-echelon faults. The fault intersection furnishes fracture permeability in the Tertiary volcanic rocks and the siliceous clastic Paleozoic rocks which underlie the volcanics. A geologic cross section (Fig. 4) shows the configuration of faulted Tertiary volcanic bedrock underlying the Whirlwind Valley alluvium.

Temperatures by chemical geothermometry of hot spring and we11 waters exceed $200^{\circ} \mathrm{C} ;{ }^{1}$ these were substantiated by temperatures measured in test wells by Magma Power Company. Hot fluid production, from the test wells, is predominantly from fractured volcanic and Paleozoic rock on the footwall side of the steeply-dipping faults of the Malpais system. 10 Chevron oil Company has recently drilled a geothermal test hole to approximately $3 \mathrm{~km}$, some $2 \mathrm{~km}$ west of the active springs area; results of drilling and well tests have not been disclosed.

Geophysical Survey. In 1973 bipole-dipole surveys covering about $100 \mathrm{~km}^{2}$ at Beowawe were completed. The surveys were conducted in Whirlwind Valley and the southward-sloping dissected volcanic tableland, south of the Malpais Escarpment. Apparent resistivity contours from a survey based on a current transmitter at a location south of the escarpment (Fig. 5) indicate a conductive zone corresponding to the present-day active hot springs and blowing wells. A more complicated resistivity pattern is obtained with the current transmitter located in Whirlwind Valley (Fig. 6). Here, prominent low resistivity zones are indicated northeast of the springs area and south of the springs, as well as in the springs area.

Planned Activities and Assessment. During 1975 further geophysical surveys are scheduled at Beowawe. Dipole-dipole traverses both parallel and perpendicular to the regional strike are required to substantiate the discreet resistivity anomalies identified earlier. Measurements will also be made to compare the telluric and induced current resistivity methods.

The primary objective of continuing geophysical measurements at Beowawe is to evaluate the effectiveness of the various reconnaissance techniques in an area in the central Basin and Range province where there is a demonstrated geothema1 resource. A secondary objective is to confirm the geophysical results with downhole information from one of the wells drilled at the site. While Beowawe is not presently being considered as a site for a test facility, no decision has been reached that forecloses such a possibility.

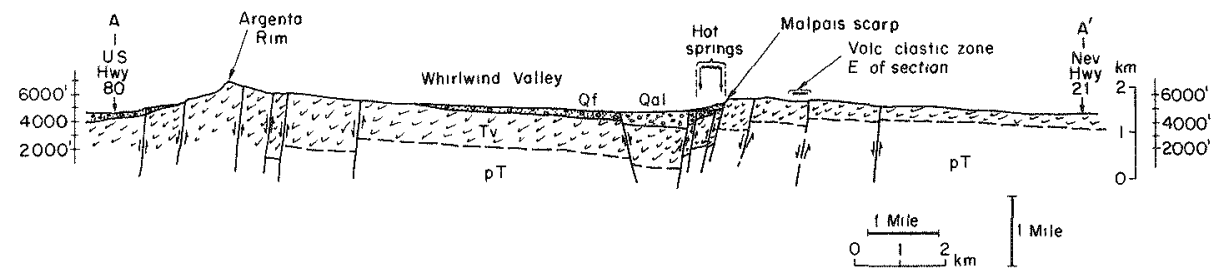

Schemaric Geologic Cross Secition A-A Whirtwind valley areo

Fig. 4. Idealized geologic cross section, Whirlwind Valley area. QaI: Quaternary alluvium, Qf: Quatemary fan deposits, Tv: Tertiary volcanic rocks, pT: pre-Tertiary rocks. 


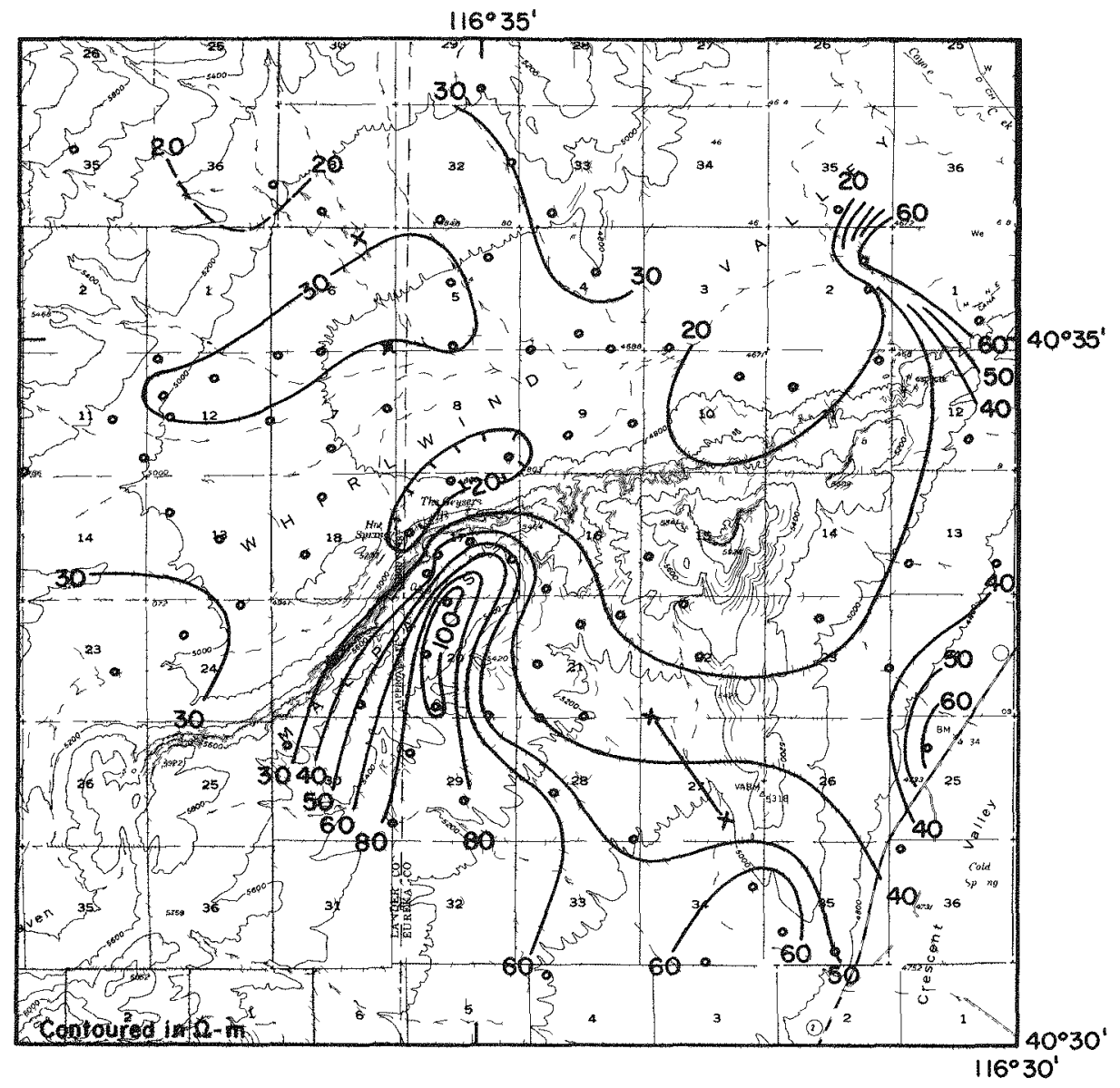

\section{BEOWAWE NEVADA \\ BIPOLE-DIPOLE RESISTIVITY \\ TRANSMITTER II}

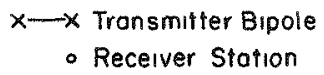

- Receiver Station

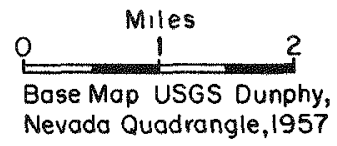

Fig. 5. Apparent resistivity contours, Whirlwind Valley areas; transmitter south of Malpais Escarpment.

(XBB 743-1631) 


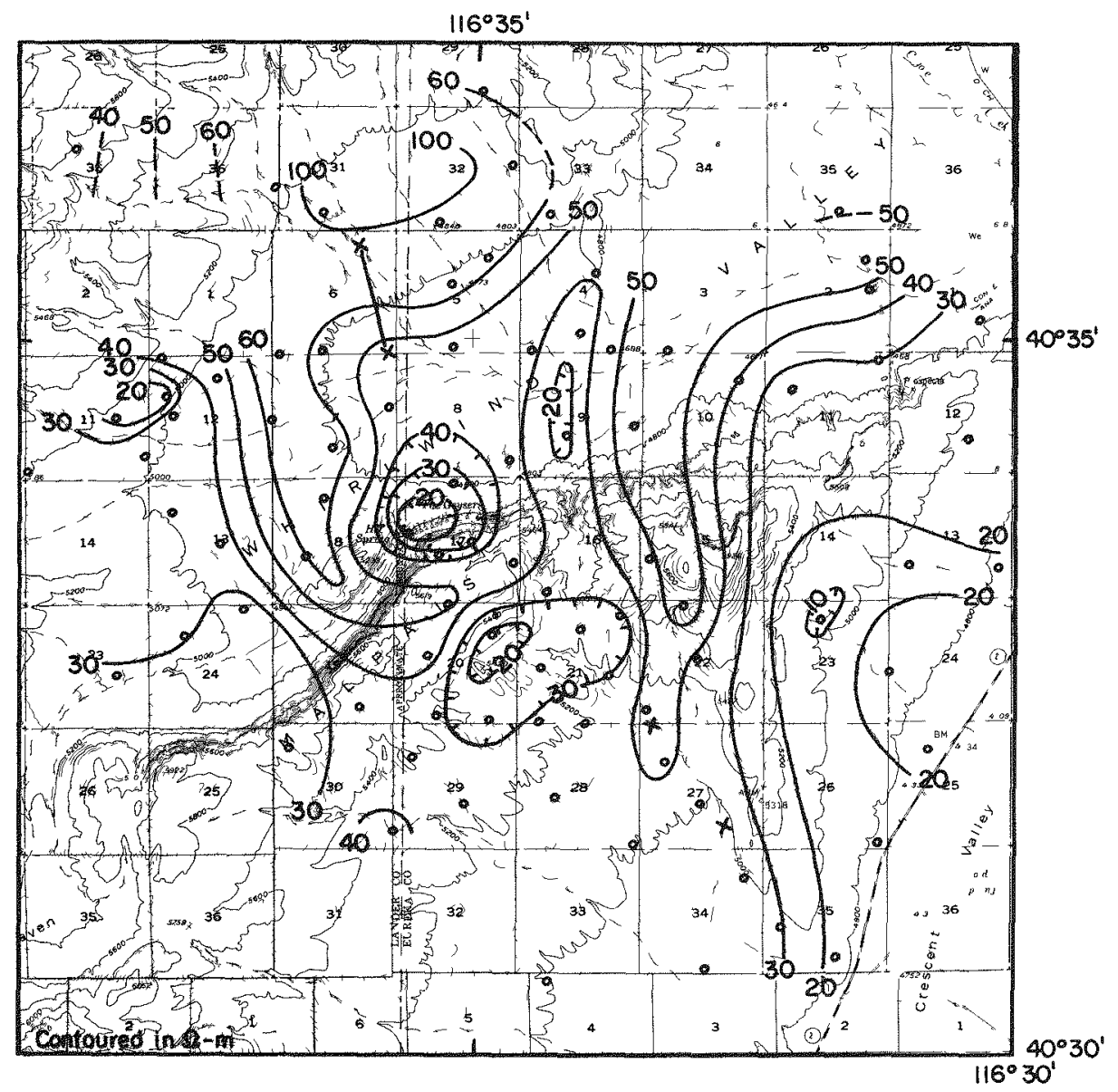

\section{BEOWAWE NEVADA \\ BIPOLE-DIPOLE RESISTIVITY \\ TRANSMITTER I}

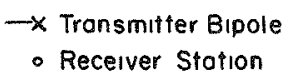

- Receiver Station

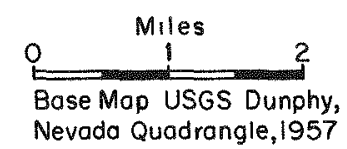

Base Map USGS Dunphy,
Nevado Quadrangle, 1957

Fig. 6. Apparent resistıvity contours, Whirlwand Valley area; transmitter in whirlwand Valley.

(XBB 743-1632) 


\section{Buffalo Valley}

Site Description and Geology. Buffalo Valley is a wel1-defined intermontane basin situated 35 to $40 \mathrm{~km}$ WSW of Battle Mountain. Its geologic setting is illustrated on the geologic map (Fig. 7) and an idealized cross section (Fig. 8). The geology has been described in detail by Noble. 11 The northern Fish Creek Mountains, bounding the valley on the east, are composed predominantly of Paleozoic clastic and Triassic carbonate rocks, overcapped by a several hundred foot thickness of
Tertiary volcanic rocks, principally ash flow tuffs of Miocene age. 12 Pliocene basaltic rocks, 2.6 to 3 million years old, were extruded from NNE-trending-basin- and range-faults which cut the earlier Tertiary and Mesozoic rocks on the east side of the valley. The basalts are exposed in wel1-preserved cinder cones. A series of prominent normal faults transects the western portion of the valley, cutting alluvial fan deposits near and at the eastern base of the Tobin Range. The Tobin Range is made up primarily of eugeosynclinal siliceous clastic rocks of Paleozoic age.

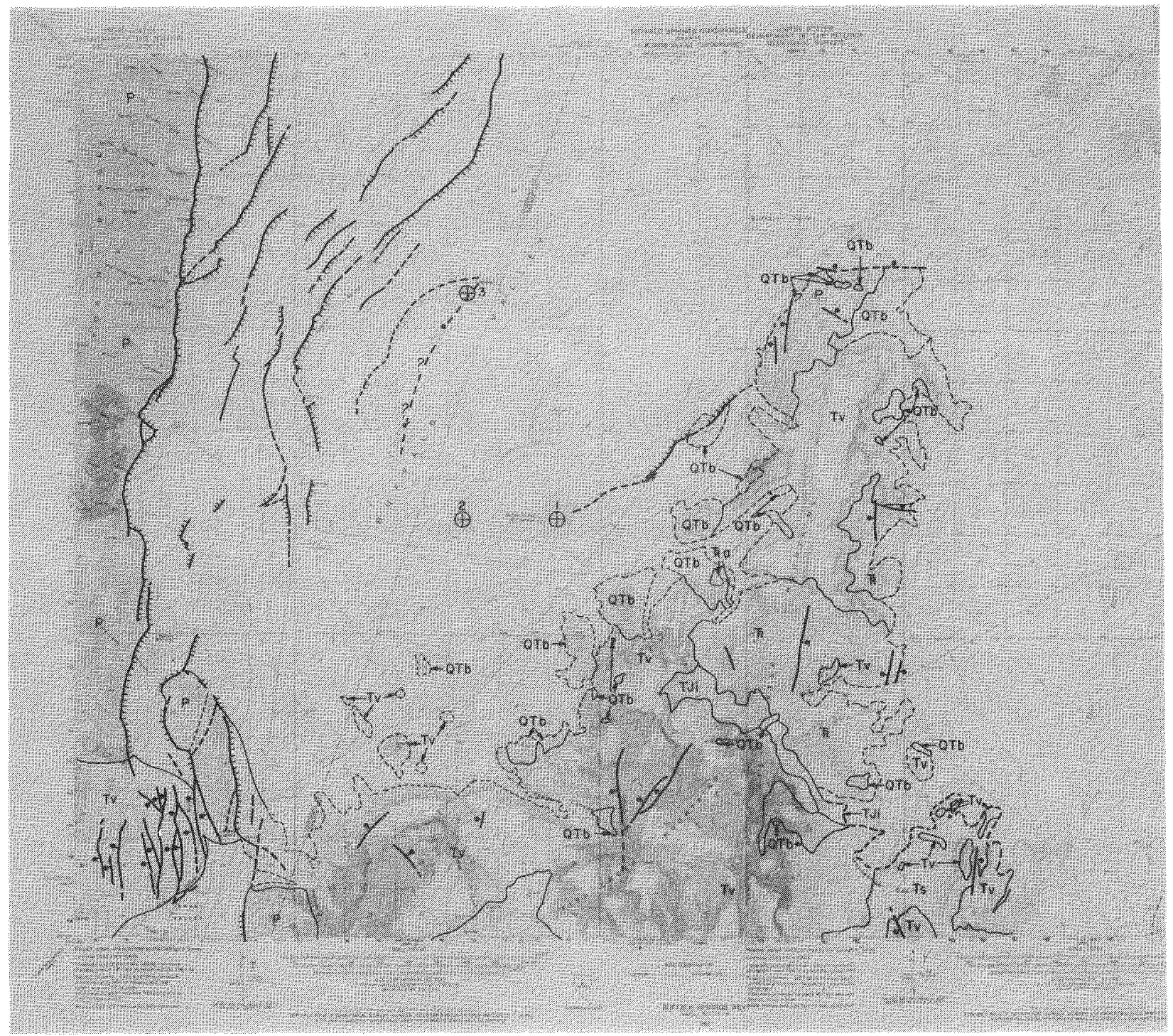

Fig. 7. Generalized geologic map of Buffalo Valley. OTb:

Quatemary-Tertiary basalt, Ts: Tertiary sedimentary rocks, Tv: Tertiary rhyolitic ash flow tuffs, TJi: granitic rock of mesozoic or Tertiary age, TR: undifferentiated Triassic sedimentary rocks, predominately carbonates, $\mathrm{P}$ : undifferentiated predominantly eugeosynclinal Paleozoic sedimentary rocks, Heavy lines: faults, balls on downthrown side. Hachured lines: observed fault scarps. Dashed heavy lines: inferred faults. Numbered crossed circles: locations of heat flow holes. 


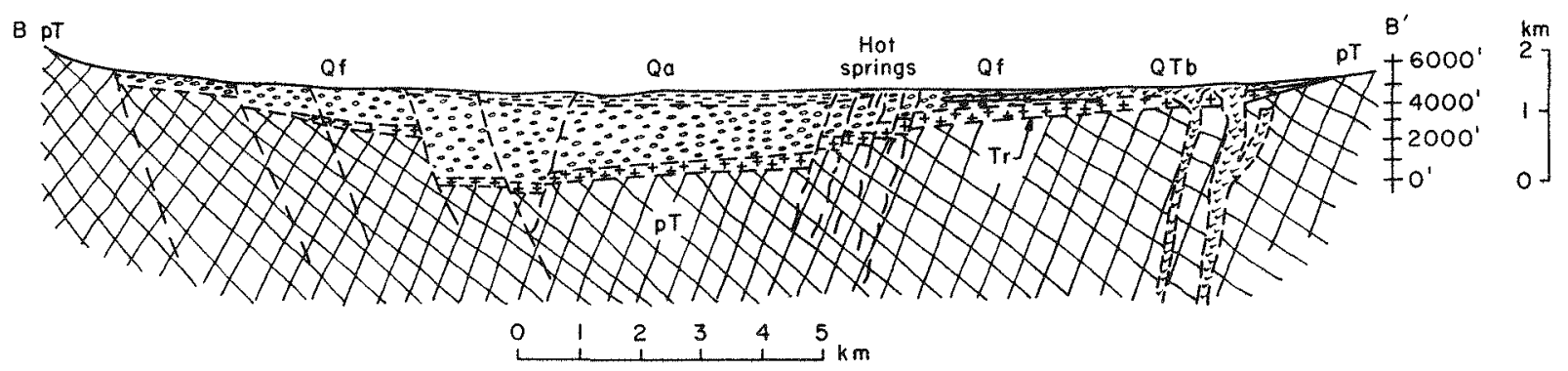

ldealized cross section $B B^{\prime}$ Buffalo Valley area

Fig. 8. Idealized goelogic cross section along B-B' (Fig. 11), Buffalo Valley area. Qa: Quaternary alluvium, Qf: Quaternary fan deposits, QTb: Quaternary-Tertiary basalt, Tr: Tertiary rhyolitic tuffs, pT: pre-Tertiary rocks.

(XBL 751-2025)

Structurally, the valley occupies an asymmetrical graben, closed at its southern end.

Gravity data, 4 shown in Fig. 9, suggests that the alluvial cover thickens progressively to 1.5 to $2 \mathrm{~km}$, west of the geographic axis of the valley, indicating that most vertical displacement occurs on the north-south-trending west-side faults. The southern margin of the graben is outlined by a series of east-west trending lineaments most of which are fault-related. South of this zone of lineaments, valley alluvium is fairly thin, covering a pediment surface on Tertiary volcanics.

Buffalo Valley hot springs are associated with a fault, extending south-westward from the Fish Creek Mountains, and with a zone of intense faulting inferred from air photos. An eastward bulge of lake beach deposits around the hot springs indicate that the springs are at least several thousand years old. Geothermometry 1 indicates subsurface temperatures of $100-130^{\circ} \mathrm{C}$ (by the $\mathrm{SiO}_{2}$ geothermometer) and about $170^{\circ} \mathrm{C}$ based on alkali element ratios corrected for Ca. Calcium carbonate is presently being deposited at the warm pools.

Airborne thermal infrared surveys disclosed a warm spring in the west central portion of the valley, approximately $5 \mathrm{~km}$ northwest of the known hot springs. Chemical analyses of this spring and others to the north in the mid-valley playa, suggest that waters with temperatures up to $150^{\circ} \mathrm{C}$ may occur at depth along a north-south-trending zone ascribed to a mid-valley fault system. Distinctive mounds and accompanying moist ground in the western portion of the Buffalo Valley playa also reflect the presence of the mid-valley fault system.

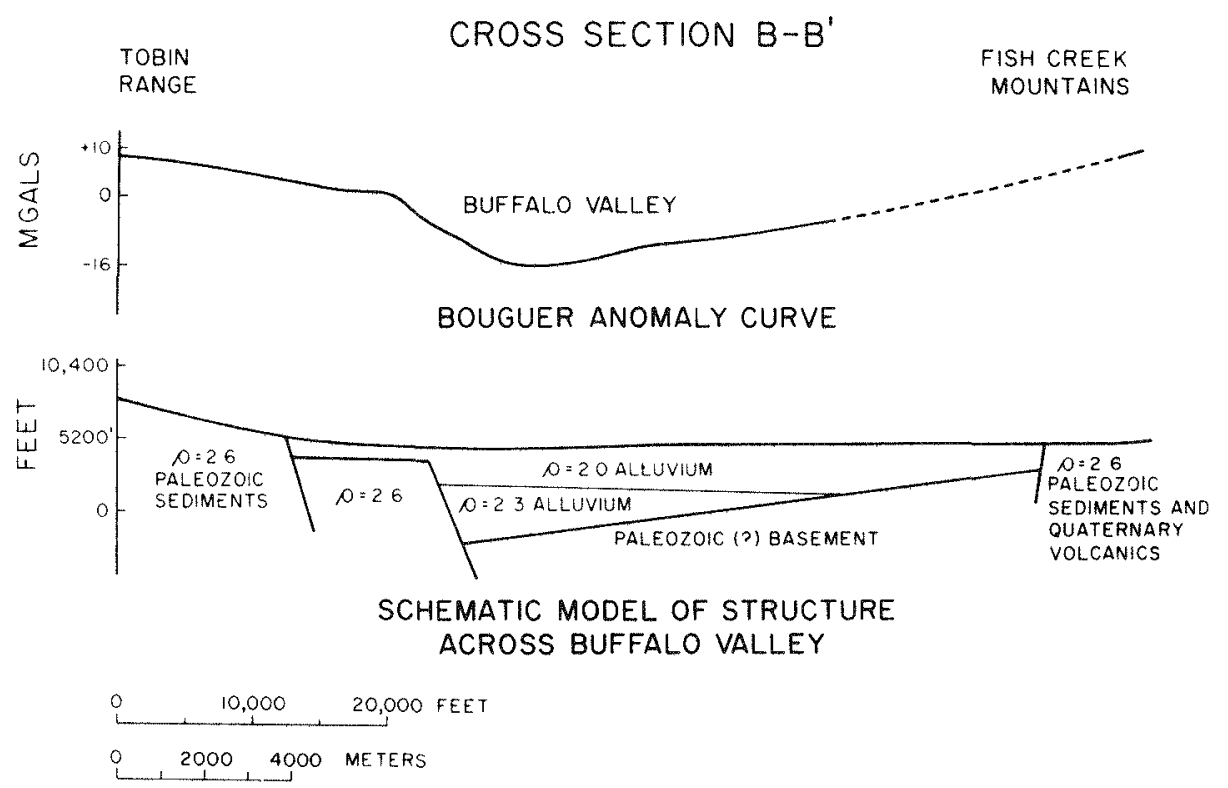

Fig. 9. Bouguer gravity profile across Buffalo Valley, after Grannell (1974). 
Geophysical Surveys: a. Resistivity, Bupole dipole resistivity surveys covering $200 \mathrm{~km}^{2}$ were completed in 1974 in Buffalo Valley. Apparent resistivity contours for current-transmitters at one of two different locations are shown on Fig. 10. The pattern indicates only rather broad areas of low apparent resistivity, probably associated with electrically conductive playa deposits.

Dipole-dipole resistivity traverse lines transecting Buffalo Valley are shown on Fig. 11. An apparent resistivity pseudo-section along $\mathrm{BB}^{\prime}$ and interpreted model of a subsurface resistivity profile are illustrated in Fig. 12. Coherent zones of low resistivity are not apparent at depth on the profile, possibly because electrically conductive near-surface alluwium inhibits penetration of electrical current to depths where conductive hot water zones exist. The profile is consistent with the geological interpretation of the area (Fig. 8). Dipole-dipole resistivity line CC', which passed through Buffalo Valley Hot Springs, indicates an associated low resistivity zone.
The use of natural electromagnetic fields is very successful in defining major conductivity varıations beneath Buffalo Valley, especially when compared to the reconnaissance dc resistivity methods. An example of this is shown in Fig. 12, where bipole-dipole, dipole-dipole, and telluric data along the line $\mathrm{BB}^{\prime}$ are compared.

A magnetotelluric profile developed by the Josephson effect magnetometer is also included in Fig. 12. A simple narrow band around the natural spectral peak at $8 \mathrm{~Hz}$ was used, and values were obtained by taking only the scalar ratio of the electric field in the profile direction to the orthogonal magnetic field. The apparent resistivities at $8 \mathrm{~Hz}$ reflect only the upper half kılometer of the section and agree very well with the short spacing dipole-dipole values.

b. Selsmic Studies. Fifty-three microearthquakes were located out of approxumately 100 detected in Buffalo Valley during a 28-day recording period in July 1974.5 Two methods of est1mating the hypocenter locations were employed; but

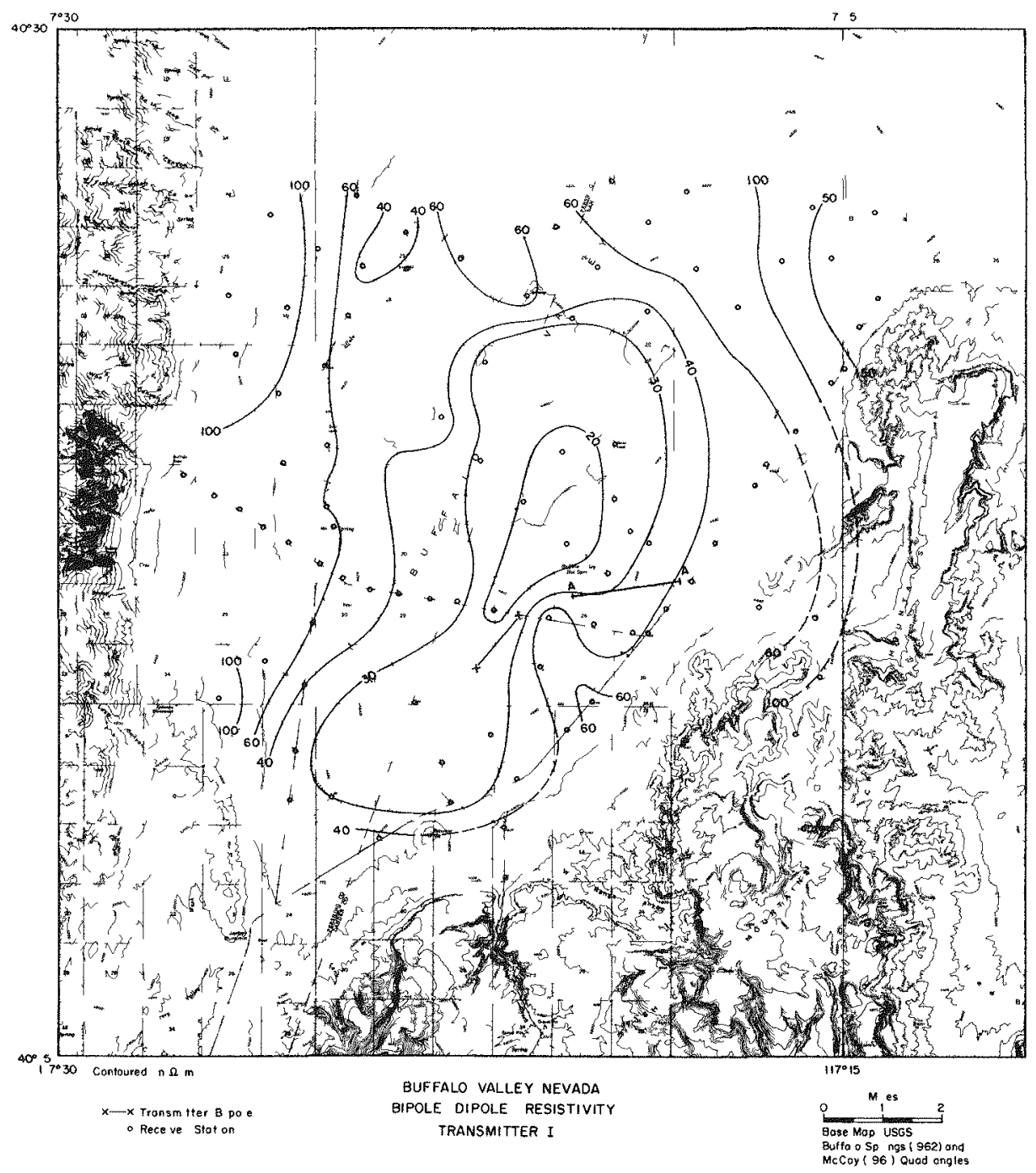

F1g. 10. Apparent resistivity contours, bipole-dipole survey at Buffalo Valley, current transmitter at location X-X. 


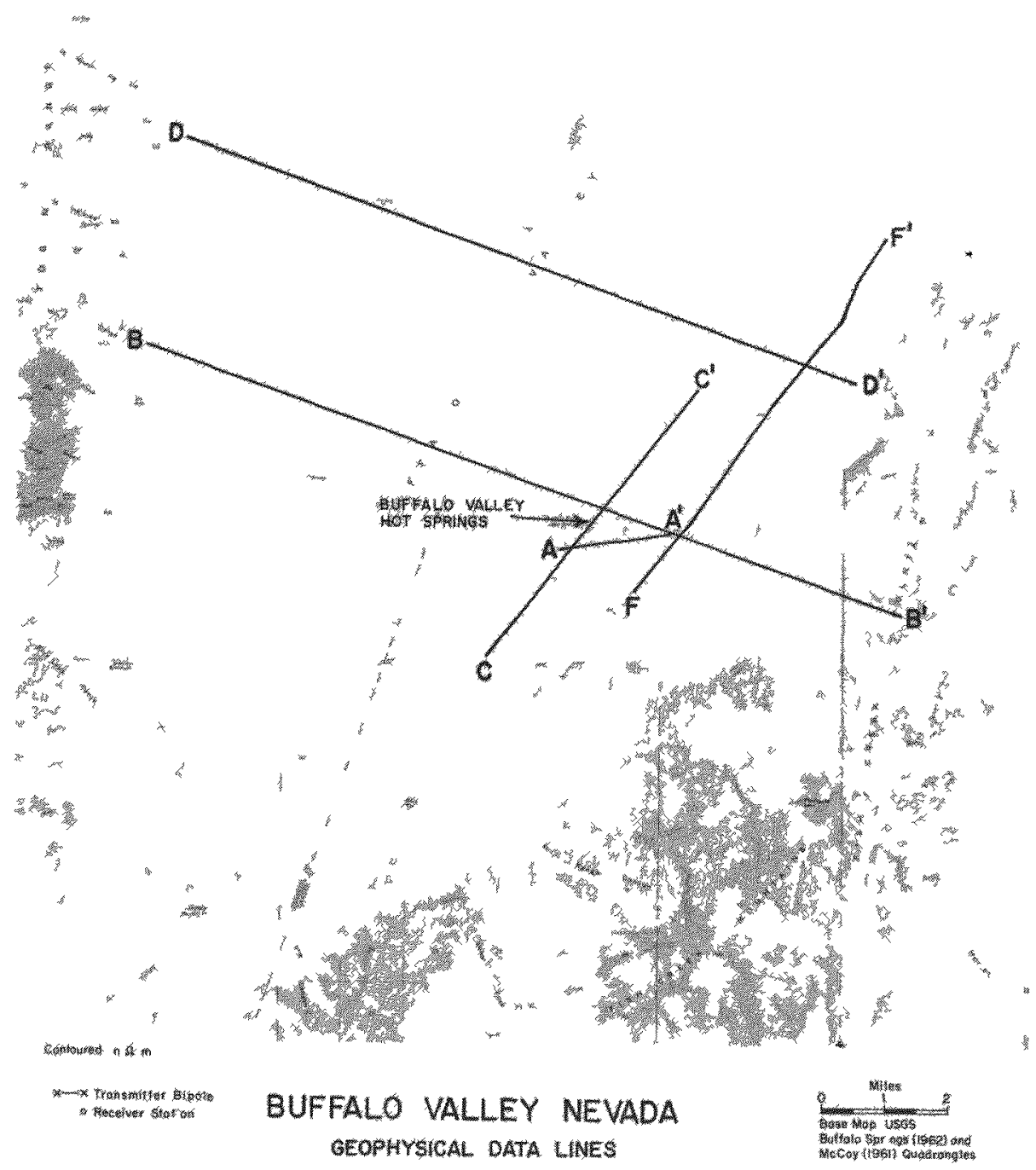

F1g. 11. Dipole-dipole resistivity profile lines, Buffalo Valley. (CBB 7410-7619)

these did not agree. One method located a cluster of shocks in the northwest quarter of the Buffalo Springs quadrangle on the west side of the valley. The other method caused the cluster to move out of the array some $8 \mathrm{~km}$ to the northwest into the Tobun Range. However, normal faulting along the west boundary of Buffalo Valley is consistent with the data.

Heat Flow 'Measurements. Three holes were drilled within the upper 100 meters of valley fı11 material at Buffalo Valley in 1974 as part of a cooperative heat flow measurement program by LBL and the U.S. Geological Survey. LBL contracted the drilling and USGS completed the holes for permanent access, obtained electrical, radioactiv Ity, and temperature $\operatorname{logs}$, and performed subsequent conductıvity measurements.

The hole locations are shown on Fig. 7 and variations of temperature with depth in Fig. 13 Hole No. 1 was drilled approxumately $500 \mathrm{~m}$ east of the eastern-most pool of Buffalo Valley Hot Springs, and an area of known warmth disclosed by previous USGS heat flow measurements. 4 The hole bottomed at 62 meters in alluvium after intersecting a $20 \mathrm{~m}$ thick zone of hard basalt, bottom-hole temperature was $125^{\circ} \mathrm{C}$ and the thermal gradient in the upper $35 \mathrm{~m}$ was $149^{\circ} \mathrm{C} \mathrm{km}-1$. Hole No. 2 was drilled about $2 \mathrm{~km}$ west of the hot springs thermal anomaly, to observe background heat flow in valley alluvium away from hot springs. The depth was about $118 \mathrm{~m}$, and the bottom hole temperature was $19^{\circ} \mathrm{C}$; observed thermal gradient was $70^{\circ} \mathrm{C} \mathrm{km}^{-1}$. Hole No. 3 was drilled on a low mound in the northwestern port of the Buffalo Valley playa at the intersection the northern projection of the mid-valley fault and the northern resistivity profile line DD'. Total depth was $98 \mathrm{~m}$, bottom hole temperature $21^{\circ} \mathrm{C}$, and themal gradient $107^{\circ} \mathrm{C} \mathrm{km}^{-1}$. 

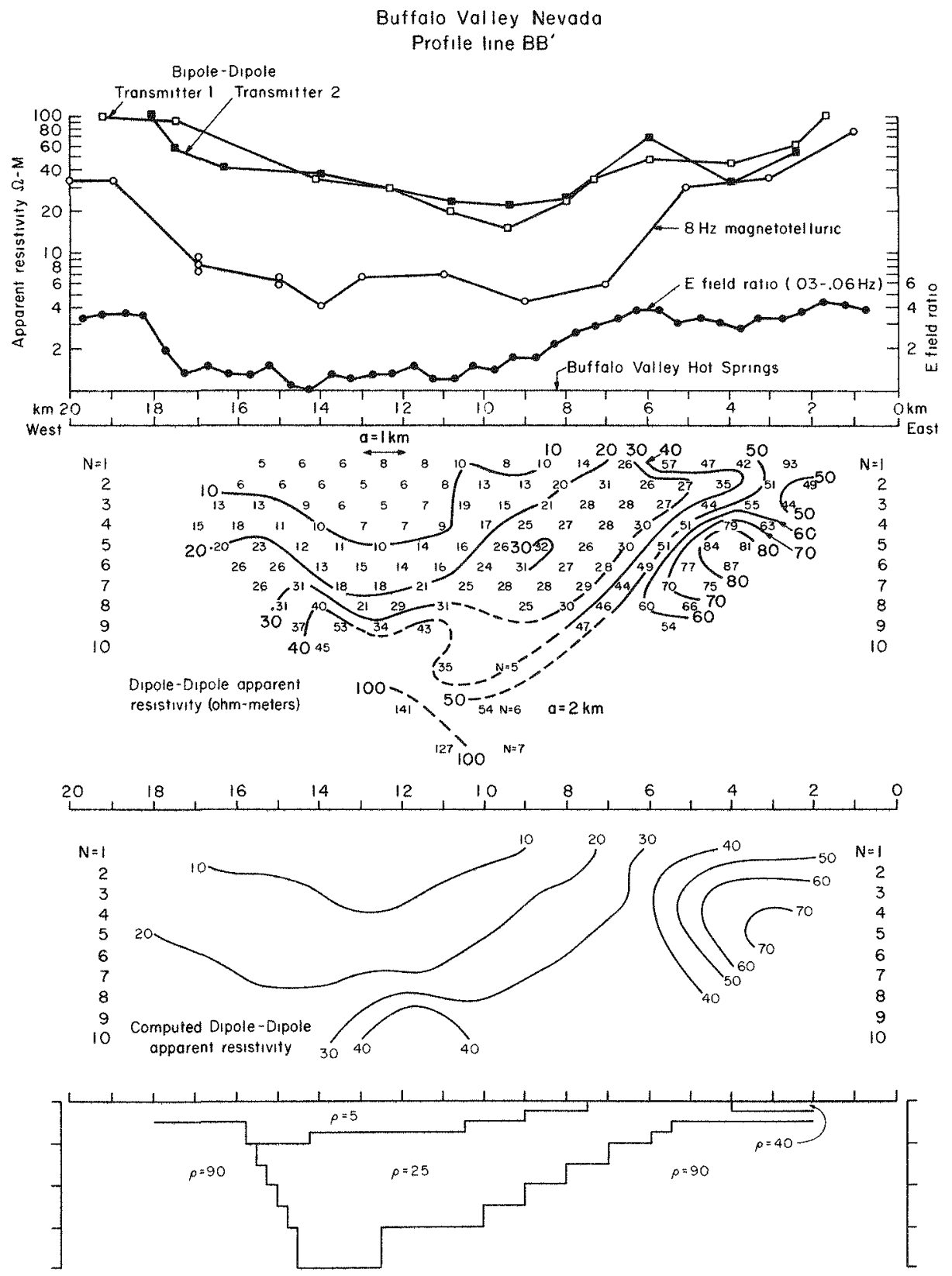

Fig. 12. Apparent resistivities, electrical field ratios, and resistivity models along profile line B-B', Buffalo Valley.

(XBL 751-2202) 


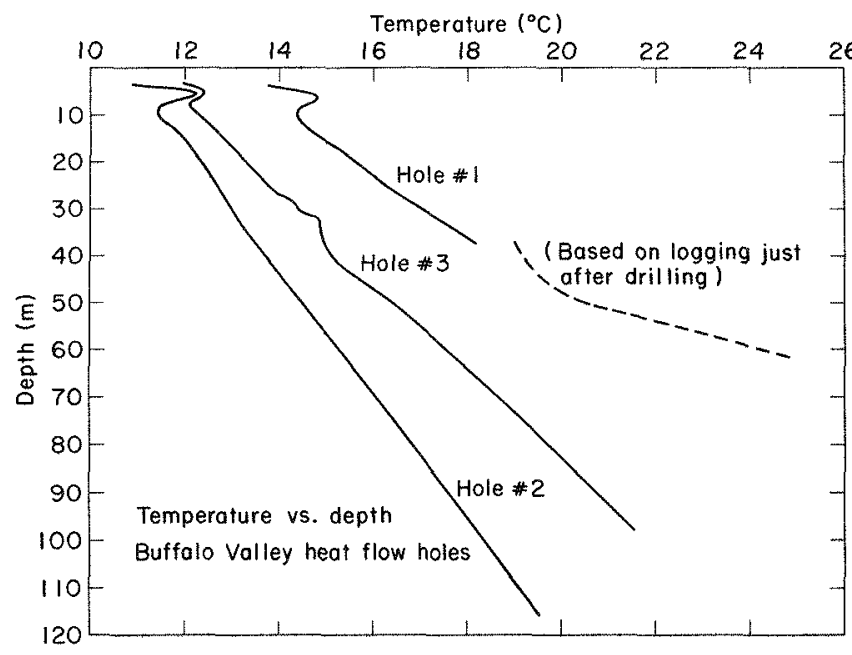

Fig. 13. Downhole temperature logs, Buffalo Valley heat flow holes.

(XBL 751-2024)

Laboratory measurements of thermal conductivities of core samples are given, together with calculated heat flow, in Table 1. The weighted mean heat flow of $3 \mathrm{hfu}^{*}$ in Hole No. 3 at the north end of Buffalo Valley playa is consistent with typical "background" heat flow for the area. 15 The value for Hole No. 2, about $2 \mathrm{hfu}$, is lower than expected for this region, especially given its much closer proximity to the active hot springs than Hole No. 3. It may be situated on or near a downward limb of convection of the hot spring system. It contrasts sharply with the relatively high value, $4.6 \mathrm{hfu}$, in Hole No. 1 which is definitely within the positive thermal anomaly associated with the hot springs.14

Table 1. Buffalo Valley Heat Flow.

\begin{tabular}{|c|c|c|c|}
\hline \multicolumn{2}{|c|}{ Hole Lithology } & $\begin{array}{l}\text { Thermal } \\
\text { conductivity } \\
\text { (m cal }{ }^{\circ} \mathrm{C}^{-1} \mathrm{~cm}^{-1}\end{array}$ & $\begin{array}{l}\text { Calculated } \\
\text { heat flow } \\
\left(\text { Hcal } \mathrm{cm}^{-2} \mathrm{sec}^{-1}\right)\end{array}$ \\
\hline \multirow[t]{3}{*}{1} & Basalt,$\rho=2$ & 3 & \multirow{3}{*}{$\begin{array}{l}4.6 \\
\text { weighted } \\
\text { mean }\end{array}$} \\
\hline & Basalt,$\rho=2.7$ & 4.5 & \\
\hline & Silty sand, $62 \mathrm{~m}$ & 2.65 & \\
\hline 2 & Clayey sand & $2.5-2.6$ & $1.98-2.05$ \\
\hline \multirow[t]{2}{*}{3} & Silty sand & $2.4-2.7$ & \multirow{2}{*}{$9\left\{\begin{array}{l}3.0 \\
6 \\
\text { weighted } \\
\text { mean }\end{array}\right.$} \\
\hline & Silty clay & $2.6-3.4$ & \\
\hline
\end{tabular}

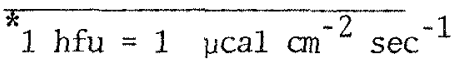

Radon Alpha-Track Survey. Alpha-track detectors for radon-222, provided by Terradex Co oration and General Electric Company, were placed in the ground near and away from radioactive warm pools in Buffalo Valley. The area is a good test site, because of the sharply varying gamma-ray fields measured at the springs. Subsequent etching of the detectors revealed high track densities at locations near the pools and a tenfold decrease in track densities in detectors away from the pools. In the southern part of Buffalo Valley, relatively high track densities reflect the proximity of the detectors to comparatively high-radioactivity rhyolitic tuffs which underlie valley alluvium and are the source of most of the alluvial material. With the exception of the hot springs area and a single site northwest of the springs, the contoured radon track density field (Fig. 14) is fairly featureless in the region surveyed. Uranium content of soil samples from track-detector holes does not correlate with alpha-track densities, indicating that a substantial portion of the radon measured comes from sources deeper than the soil surrounding the detectors. This work is reported in detai1 by Wollenberg. 16

Chemistry. The chemical composition of four separate hot pools at Buffalo Valley hot springs was determined. The compositions of all four pools are very similar, and suggest a common origin.

Preliminary Conclusions. To date, Buffalo valley has received the most extensive evaluation of the four sites selected for detailed investigation. However, no discreet geothermal targets were identified and over half the land studied has been ruled out from further consideration as a geothermal resource area. All surface electrical data, shallow heat flow holes, and radiometric data indicate, that if a geothermal resource exists at a11, it must be diffuse and of fairly low temperature.

Because one of the objectives of the site evaluation program is to compare the effectiveness of different geophysical methods in identifying geothermal resources, a deep hole is planned at Buffalo Valley to confirm the results obtained so far. This hole would be small bore ( 5 to 6 inch diameter) and would penetrate at least 300 meters into bedrock below the valley fill alluvium. The site location of this hole has yet to be determined.

Grass Valley (Leach Hot Springs)

Site Description and Geology. A potential geothermal resource area in Grass Valley is 10cated in the vicinity of Leach Hot Springs, approximately $50 \mathrm{~km}$ south of Winnemucca. The Sonoma and Tobin Ranges bound the valley on the east, while the valley is constricted south of the hot springs by the Goldbanks Hills, locus of earlier mercury mining. Grass Valley is bounded on the west by the basalt-capped East Range. The distribution of major 1ithologic units in the region is illust on the geologic map (Fig. 15) and their stratigraphic relationships on the cross section (Fig. 16b). Paleozoic siliceous clastic rocks and greenstones are the oldest bedrock types in the region. In places in the Sonoma and Tobin Ranges, the 


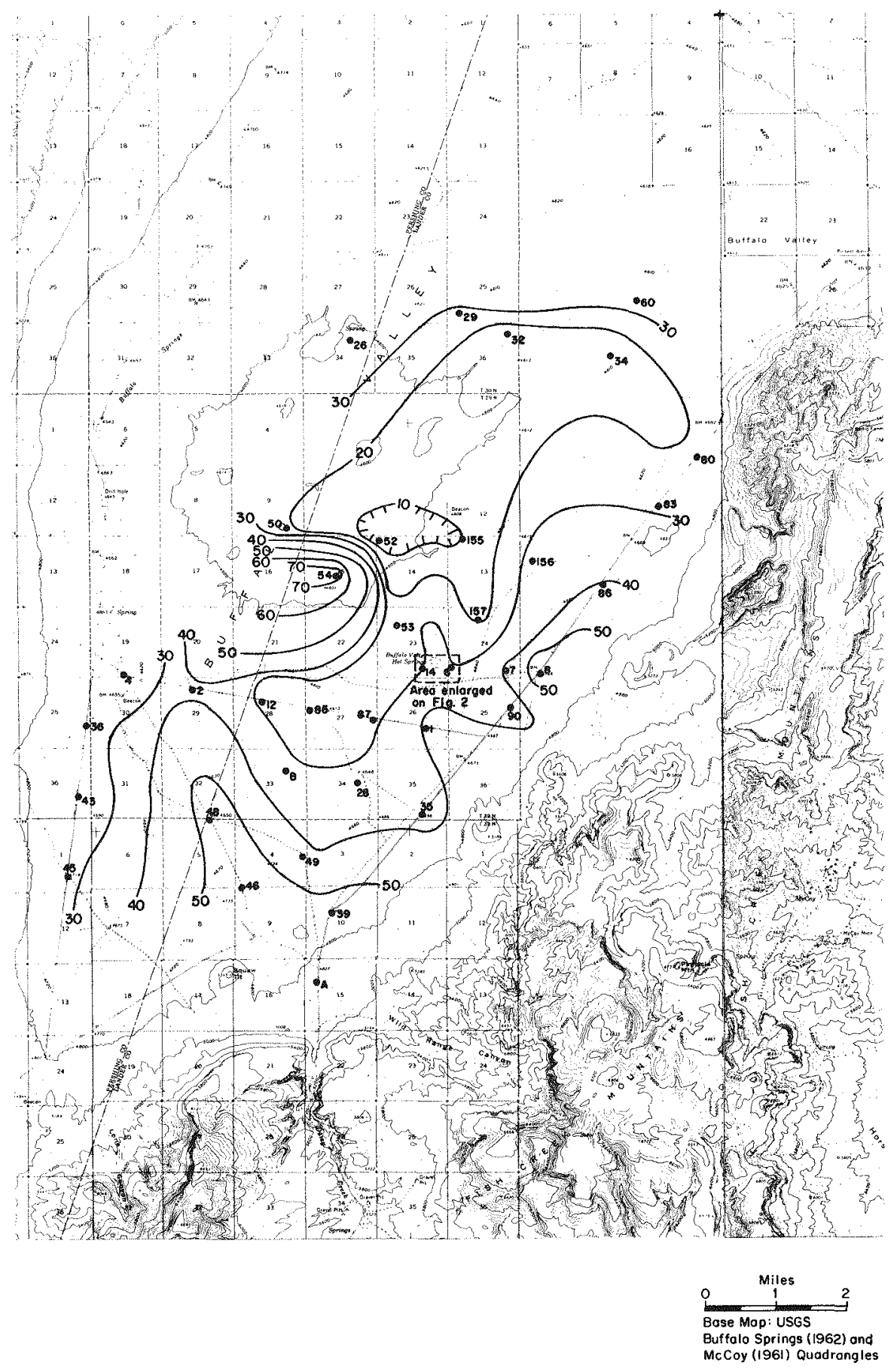

Fig. 14. Contours of radon alpha-track density (in tracks $\mathrm{mm}^{-2}$, norma1ized to a 30-day exposure) in Buffalo Valley.

$(\mathrm{XBB} 7412-8765 \mathrm{~A})$ 


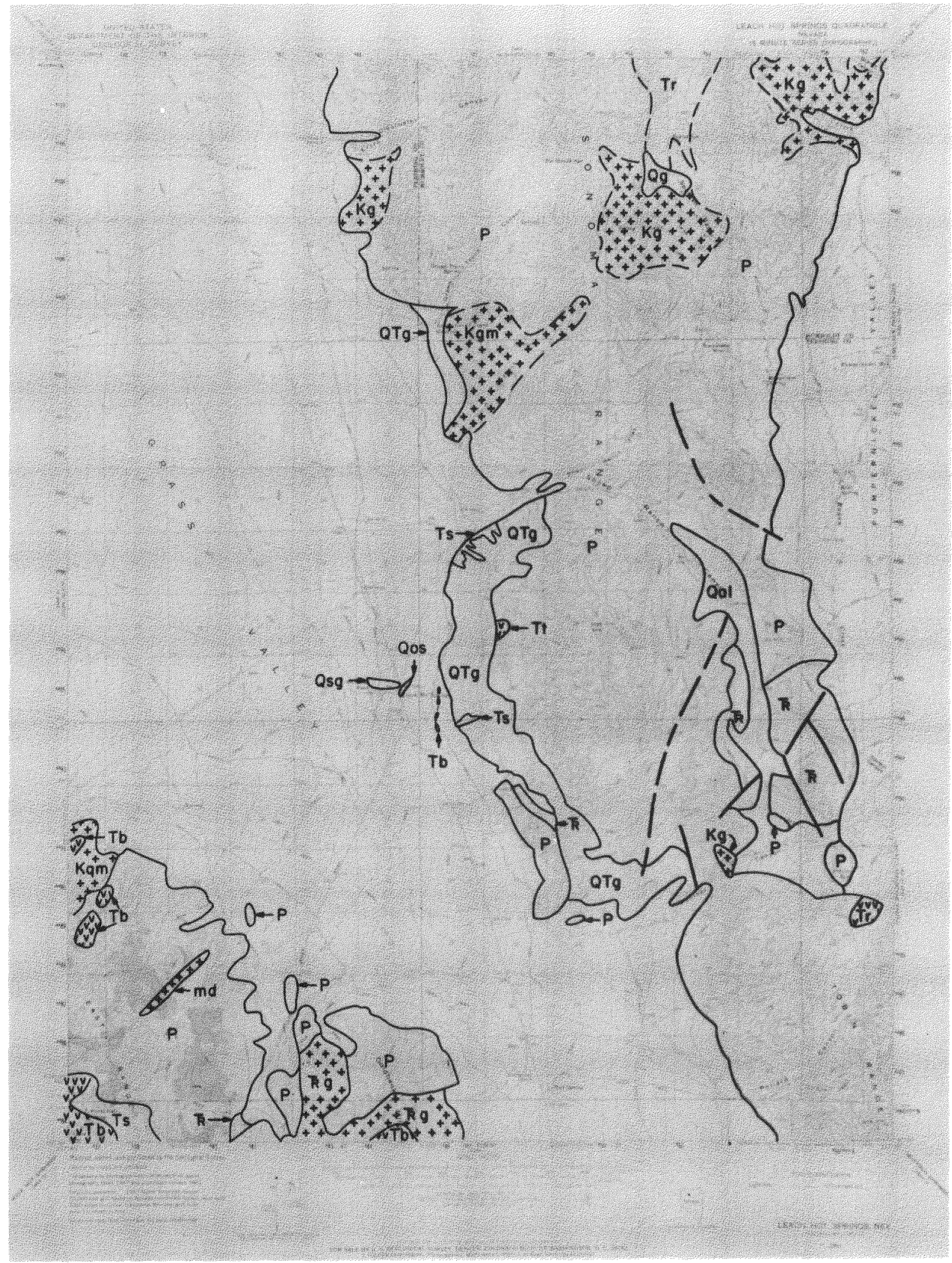

Fig. 15. Lithologic map, Leach Hot Springs area. Qa1: alluvium, Zos: older sinter deposits, Qsg: sinter gravels, QTg: Quaternary-Tertiary grave1s and fanglomerates, Tb: Tertiary basalt, Tr: Tertiary ryholite, Tt: tuff, Ts: Tertiary sedimetary rocks, Kgm: quartz monzonite, $\mathrm{Kg}$ : granitic rock, md: mafic dike, TRg: Triassic granitic rocks, TR: undifferentiated Triassic sedimentary rocks, P: undifferentiated Paleozoic sedimentary rocks. 




F1g. 16a. Electrical geophysical survey 1ines, Grass Valley. (CBB 7410-7621)

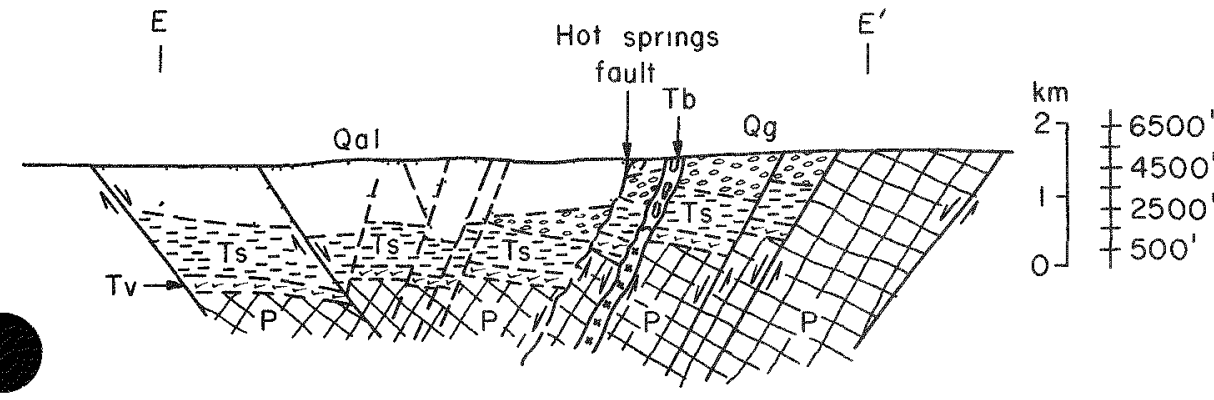

Idealized cross section $E E^{\prime}$

Leach Hot Springs area
Fig. 16b. Idealized goelogic cross section along E-E' (Fig. 16a) Leach Hot Springs area.

Qal alluvium, Qg. gravel and fanglomerates, $\mathrm{Tb} \cdot$ basalt, Ts: Tertiary sedimentary rocks, Tv: Tertiary tuffaceous rock, $P$. undifferentıated Paleozolc sedumentary rocks.

(XBL 751-2026) 
Paleozoics are in thrust-fault contact with Triassic siliceous clastic and carbonate rocks. The Paleozoic and Triassic rocks have been intruded by granitic rocks, of probable Triassic age in the Goldbanks Hills; elsewhere the granitics are probably of Cretaceous age. Though not exposed in the Leach Hot Springs area, Oligocene-Miocene rhyolitic tuffaceous rocks are probably present in the subsurface. They are overlain by interbedded sandstone, fresh water limestone and altered tuffs, and by coarser conglomeratic sediments (fanglomerates) derived from mountain range fronts steepened by the onset of basin-and-range faulting. The fanglomerates are opalized in places by siliceous hydrothermal activity associated with fault zones; occasionally the locus of mercury mineralization. Opalization of mercury deposits in the Goldbanks Hills and East Range closely resembles the opalized sinter at Leach Hot Springs. The Tertiary sedimentary sequence is overcapped by predominantly basaltic volcanic rocks whose ages range from 14.5 to 11.5 million years.

Leach Hot Springs is located on a fault, revealed by a 10 - to $15-\mathrm{m}$ high scarp trending $\mathrm{NE}$. Normal faulting since mid-Tertiary has offset rock units vertically several tens to several hundred meters, as shown in the cross section, Fig. 16b The hot springs occur at the zone of intersection of the NE trending fault and NNW-SSE trending 1ineaments. The zone of intersecting lineaments and scarplets southwest of leach Hot Springs, between the springs and the Goldbanks Hills, corresponds to an area of appreciable microearthquake activity, 5 suggesting that active faulting may be associated with hydrothermal activity.

Total surface flow from the Leach Hot Springs system has been measured at $130 \mathrm{lmin}-1.17$ Surface temperatures of the springs reach the boiling point $\left(94^{\circ} \mathrm{C}\right)$. Water temperatures at depth are estimated. to be 155 to $170^{\circ} \mathrm{C}$, based on silica and alkali-element geothermometers. 1 Material deposited by Leach Hot Springs, presently and in the past, is predominantly silica.

Geophysical Surveys: Resistivity, About 75\% of the area selected for investigation at Leach Hot Springs was surveyed by the bipole-dipole resistivity method. Excellent agreement between this method and a telluric current reconnaissance technique was obtained on 76 line $\mathrm{km}$. In addition, a short dipole-dipole line with dipole lengths of 250 meters and separation of up to $35 \mathrm{~km}$ was run on an east-west profile line, shown in Fig. 16 . The results are shown in Fig. 17.

The surveys show a complex resistivity distribution along the east side of Grass Valley in the vicinity of Leach Hot Springs. An example of a satisfactory model for this line is shown on Fig. 17. Several strong conductivity contrasts associated with intersecting faults are promising reservoir targets in this area.

Seismic Studies. Thirty-five microearthquakes were located with an array in Grass valley. 5 The microearthquakes seem to occur in a triangular region bounded by Leach Hot Springs, the Goldbanks Hills and Pollard Canyon. The fault plane solutions indicate nomal faulting striking
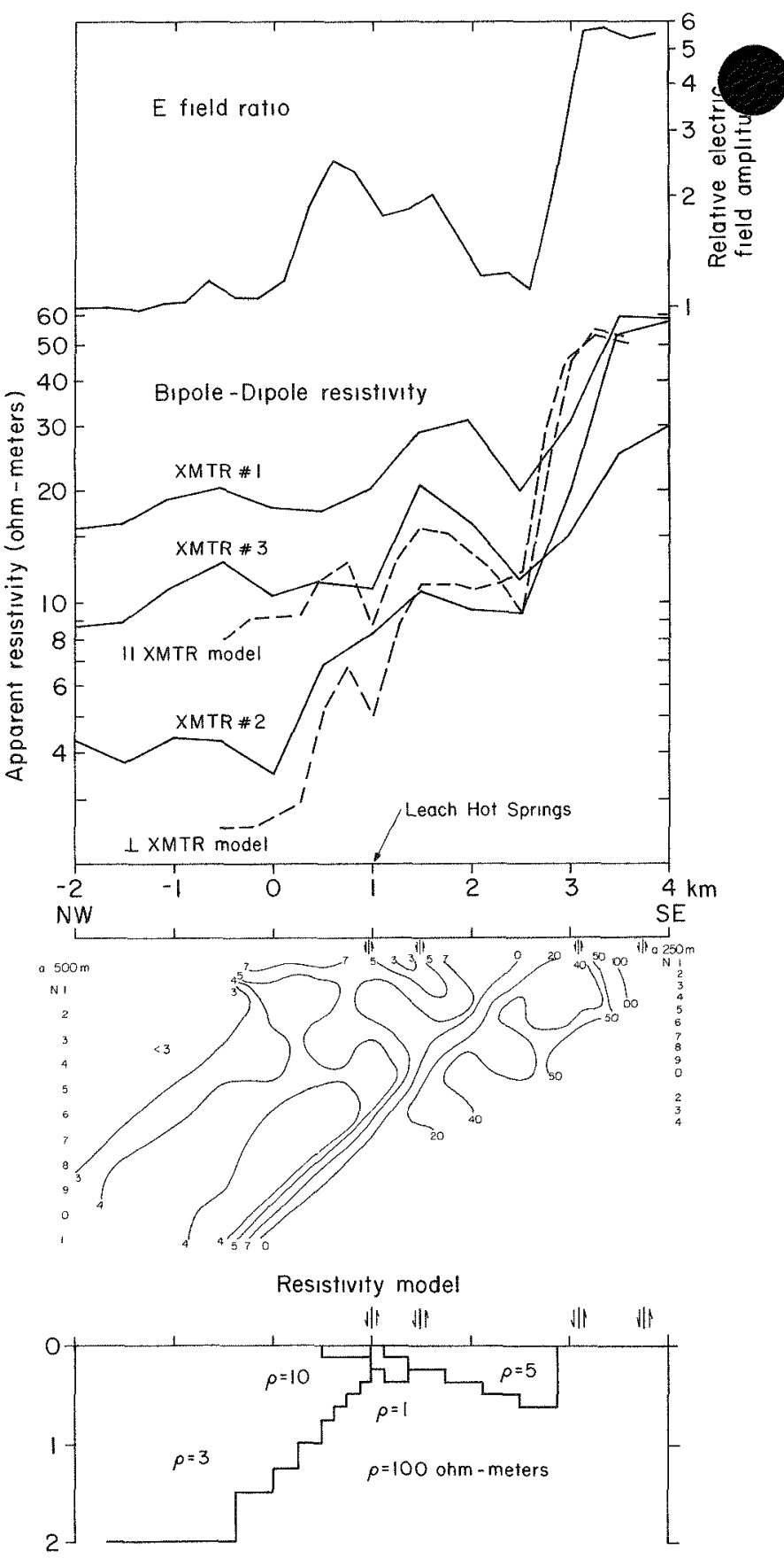

Fig. 17. Electrical field ratios, bipole-dipole profiles, and apparent resistivity mode1, along E-E', Grass Valley.

(XBL 751-2203)

from SW to NE through Leach Hot Springs dipping $45^{\circ}-50^{\circ}$ to the SE. To check the spatial relationship of this cluster and another in Buffalo Valley, one large-scale array was installed reoccupying several selected stations in Buffalo Valley and Grass Valley, plus an additional station in Pleasant Valley. This configuration enabled simultaneous recording of the larger events in the three va

Five events were recorded and located in a four day period. All the epicenters were in the triangular cluster area found earlier in the Grass Valley area. 
Conclusions and Planned Activities. The geophysical surveys have demonstrated the capabilities of telluric versus induced current resistivity methods in Grass Valley. Grass Valley is a more promising resource area than Buffalo Valley because discreet resistivity zones have been identified at depth. Microearthquake activity is also present in Grass Valley, but its absent in Buffalo Valley.

Field reconnaissance activities at Grass Valley in 1975 will rely heavily on telluric methods because of their proven capabilities. A detailed dipole-dipole survey to identify target areas for drilling will follow, using the telluric data as a guide.

\section{Buena Vista Valley (Kyle Hot Springs)}

The Kyle Hot Springs area of Buena Vista Valley is situated 35 to $50 \mathrm{~km}$ south of Imlay, a crossroads on Interstate 80 approximately $40 \mathrm{~km}$ south of Winnemucca. The area occupies a large eastward "embayment" of Buena Vista Valley into the Sast Range, accentuated by the east-west buttress of Granite Mountain on the south.

Air photo and on-site observations by Noble, 8 indicate that Kyle Hot Springs are localized by intense faulting and fault intersections. A prominent system of normal-fault scarps follows the western front of the East Range, northward from Granite Mountain, passing east of Kyle Hot Springs. Another prominent fault system trends southward from near the mouth of Klondike Canyon, thence southwestward to pass through the active hot springs. This system continues southwestward through an old, now inactive, spring area. The active and inactive spring areas at Kyle, then, are situated within a belt about $2 \mathrm{~km}$ long by $1 \mathrm{~km}$ wide which contains an unusually large number of faults and fault intersections. Another intense zone of intersecting faults is apparent in alluvium at the western end and northwestern edge of Granite Mountain. However, fracturing here does not appear as intense as at Kyle Hot Springs; evaluation of geothermal potential must await comparison of geophysical data from the two areas.

The hot springs at Kyle consist of quiescent pools, developed for bathing and "steaming." Maximum surface water temperature is $77^{\circ} \mathrm{C}$; total outflow is estimated at $20^{\circ} \mathrm{l} / \mathrm{min}$, and temperatures at depth within the spring system are expected to be between 170 and $190^{\circ} \mathrm{C}$, based on chemical geothermometers. 1 The active hot springs are presently depositing predominantly $\mathrm{CaCO}_{3}$, though opalized sinter is abundant in older deposits and predominates at the inactive "fossil" hot spring area.

A microearthquake study has been carried out in the Buena Vista Valley, using an eight-station detector array. In contrast to Grass Valley, no appreciable microseismic activity was detected.

\section{Planned Activites}

The zone of intersecting faults at Kyle Hot Springs is favorable for the identification of subsurface targets by geophysical methods. Another zone of intense faulting at the northwestern edge of Granite Mountain also appears favorable. During 1975, an intensive geophysical reconnaissance program will be conducted in Buena Vista Valley in the vicinity of these two sites.

\section{SUMMARY}

Fiedd activities in four potential geothemal resource areas in north-central Nevada in 1974 have emphasized geophysical techniques. Comparison of electrical resistivity measurements by induced and natural field methods indicate that telluric and/or magnetotelluric techniques may be more economic and efficient than bipole-dipole surveys in the reconnaissance phase of the program. Geologic and geophysical surveys of hot spring areas in Buffalo and Grass Valleys indicate that the geothermal resource potential of the Leach Hot Springs area in Grass Valley is greater than that of Buffalo Valley. Preliminary geologic reconnaissance of the Kyle Hot Springs area in Buena Vista Valley also suggests an appreciable geothermal potential in that region.

Geochemical sampling and analyses of coldand hot-spring waters and related rock units have yielded trace-element affinities that may be diagnostic in determining the pathways of waters from their source into geothermal systems.

Activities in 1975 will include detailed dipole-dipole resistivity transects of Grass and Whirlwind Valleys, preliminary heat-flow drilling in Grass Valley, and electrical and natural-field surveys of the Buena Vista Valley area. These studies, besides providing a continuing evaluation of techniques, will lead to location of sites for deep calibration holes. Lithologic, hydrologic, and geophysical data from these holes will be used to compare subsurface conditions with those predicted by surface surveys.

\section{REFERENCES}

1. R. H. Mariner, et al., The chemical composition and estimated minimum thermal reservoir temperatures of the principal hot springs of northern and central Nevada, U. S. Geological Survey open file report (1974).

2. J. Quade and D. Trexler, An analysis of the airborne data from NASA's Mission No. 268 over geothermal areas of northern Nevada, report to the LBL Geotherma1 Group (1975).

3. R. Corwin, The use of the self potential method for geothermal exploration; report to LBL Geothermal Group (1974).

4. R. B. Granne11, A regional gravity survey of the Fish Creek Mountains and surrounding area, north-central Nevada, Abs. Geol. Soc. Amer. Cordilleran Section $71 \mathrm{st}$ annual $\mathrm{mtg}$. (1974).

5. E. Majer, and T. V. McEvilly, Seismic reconnaissance in Nevada, report to LBL Geothermal Group (1974).

6. A. Hebert and K. Street, Ana1. Chem. 46, 203 (1974) .

7. H. Wollenberg, Geophys. Res. Lett. 1 (8) 359 (1974).

8. H. Wollenberg and A. R. Smith, Proc. 2nd Symp. on the Natural Radiation Enviroment (in press). 
9. W. A. Desterling, Areal geology of the Geysers and vicinity, Eureka and Lander Counties, Nevada; geologic map, Southerm Pacific Co. Mineral Resources Survey, 1960.

10. W. A. Oesterling, Reports and 1ithologic logs of Beowawe test wells, to Sierra Pacific Power Co. (1964, 1965).

11. D. C. Noble, Reconnaissance geological study of the Buffalo Valley area, northern Nevada, report to LBL Geothermal Group (1974).

12. E. H. McKee, U. S. Geol. Surv. Professional Paper 681 (1970).

13. E. H. McKee, and Archibald, private communication, (1974).
14. F. Olmsted, Private commication (1974).

15. J. H. Sass, et al., Jour. Geophys. Res. 76 (26), 6376 (1971).

16. H. Wollenberg, Radon alpha-track survey of a potential geothermal resource area, LBL-3225 (1974).

17. F. H. Olmsted, P. A. Glancy, J. R. Harri11, F. E. Rush and A. S. Vandenburgh, U. S. Geol. Surv, open file report $75-56$ (1975).

18. D. C. Noble, Reconnaissance study of the geological setting and fault control of the Kyle Hot Springs geothermal area; report to LBL Geothermal Group (1974).

\title{
EXPERIMENTAL POWER GENERATION FACTLITY
}

\author{
K. Mirk and W. Kleck
}

The magnitude of geothermal resources appears to be substantial, particularly in the western region of the United States. The report to the President by the Chairman, Atomic Energy Commission ${ }^{1}$ estimates that 20,000 MW of geothermal electrical generating capacity could be on-line in this country by 1985 and $80,000 \mathrm{MW}$ by the year 2000 . The present total installed electrical generating capacity is approximately $400 \mathrm{MW}$.

Lawrence Berkeley Laboratory has formulated a program to develop the potential of 1iquid-dominated hydrothermal resources in the moderate temperature, low salinity brine category. A major facet of this program is the construction and operation of a $10 \mathrm{MW}$ experimental power generation facility. This project is intended to demonstrate the technical and economic feasibility of the large-scale commercial use of geothermal brines in the temperature range of $170^{\circ} \mathrm{C}$ to $210^{\circ} \mathrm{C}$ and with a salinity of less than 5,000 ppm total dissolved solids.

We have analyzed the cycle efficiency and cost of various types of power cycles that might be used in the pilot plant. One system, the binaryfluid cycle, appears to be best suited to brines in this temperature range. A secondary fluid with a low temperature boiling point is pumped through a heat exchanger where it absorbs heat from the brine and changes to a high pressure vapor. This vapor ex. pands through a turbine converting its thermal energy to mechanical energy and passes into a condenser where additional energy is absorbed, changing the vapor back into a liquid. A pump returns the condensate to the heat exchanger, completing the cycle.

A conceptual design of the $10 \mathrm{MN}$ pilot plant with a binary power cycle has been completed. 2 This design assumes isobutane as the secondary fluid and $a$ wellhead brine temperature of $200^{\circ} \mathrm{C}$ at a site in either Buffalo, Grass, or Buena Vista Valleys in Nevada.

Figure 1 shows a simplified flow diagram of this pilot plant design. Total net electrical output is $7,900 \mathrm{~kW}$. The estimated themal efficiency is $15 \%$.

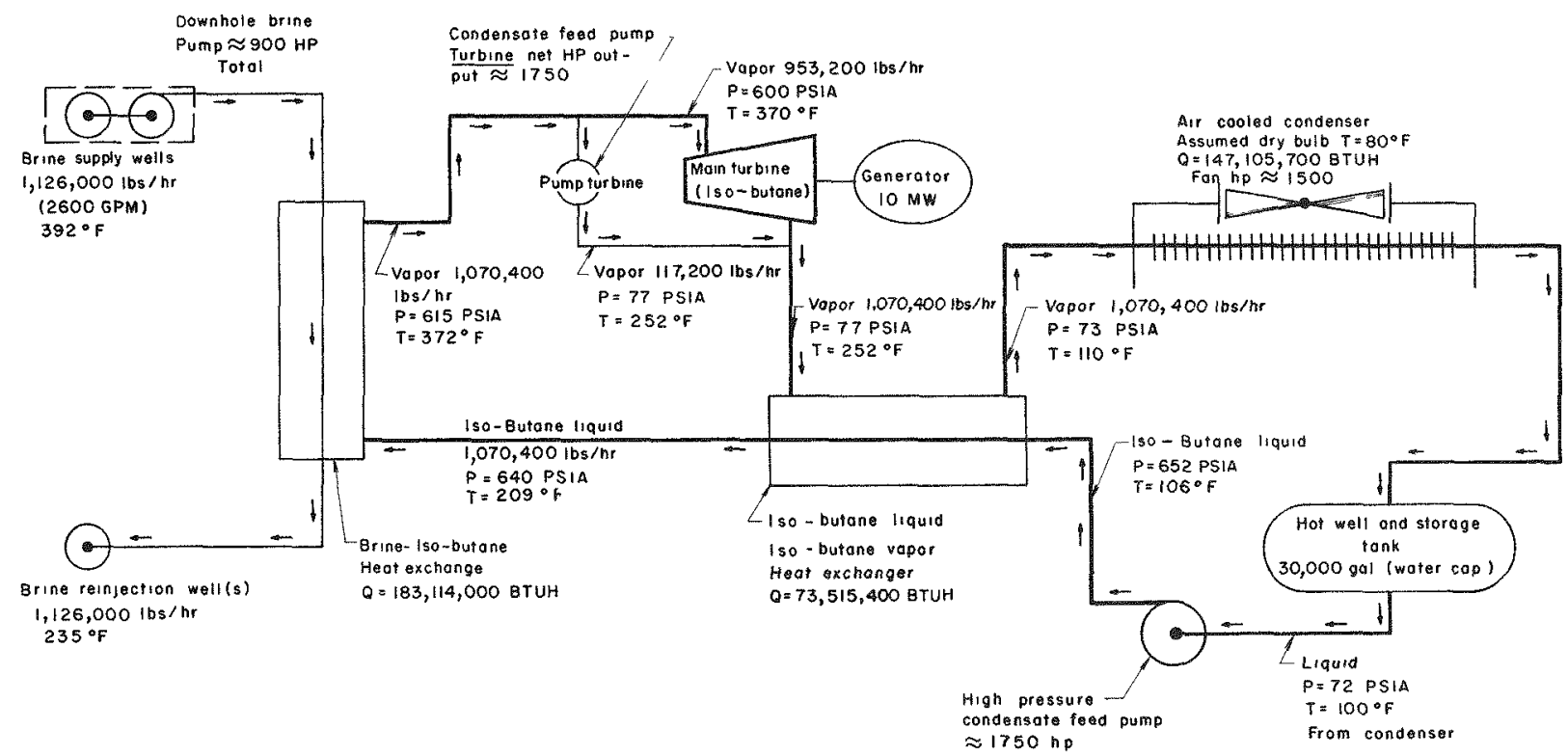

(XBL 749-4193)

Fig. 1. Flow diagram for $10 \mathrm{MW}$ geothermal power plant. 
Three production wells furnsh the required rine flow and two reinjection wells return the rine to the deep underground reservoir system. A downhole pump in each production well provides sufficient pressure to lift the brine, prevent flashing, maintain dissolved $\mathrm{CO}_{2}$ in solution, and overcome friction losses in the heat exchangers and reinjection wells.

The heat exchangers consist of eight shelland-tube type units comected in series. Each unt has a shell diameter of five feet and a tube length of twenty feet. Manifolding allows any pair to be isolated for cleaning without interrupting plant operation. We assumed an average heat transfer coefficient of $87 \mathrm{Btu} / \mathrm{ft}^{2} \times \mathrm{hr} \times{ }^{\circ} \mathrm{F}$.

Two condensate feed pumps, each powered by an isobutane turbine, supply sufficient head to allow the system to operate in the supercritical region. This minimizes the "pinch-point" effect in the brine/isobutane heat exchanger.

An isobutane vapor/isobutane liquid, regenerative heat exchanger transfers a large amount of superheat from the turbine exhaust to the Isobutane fed to the main heat exchanger.

We regulate brine flow through the heat exchanger to maintain a turbine inlet temperature of $370 \mathrm{~F}$. We selected a radial inflow turbine for the conceptual design. It is a single stage unit with an impeller diameter of 23 inches, which rotates at 9,000 rpm. A reduction gear comects the turbine to the generator which turns at 3600 rpm. The turbo-generator rating is $10 \mathrm{MW}$; downholo pumps, feed water pumps, cooling tower fans, etc., consume $2.1 \mathrm{MW}$ tota1.
A dry-type cooling tower/condenser is used. The initlal cost is higher than the required for a wet-type cooling tower and the plant efficiency is lower due to the large fan power requirements. However, both maintenance and enviromental problems are reduced with the use of a dry-type tower. We assumed a dry bulb temperature of $80^{\circ} \mathrm{F}$. Condensate returns by gravity from the condenser section to the hot well.

An automatic control system allows the plant to be operated from a remote station anywhere within the power distribution system. All plant operations, except for intlal startup, can be performed by this system.

Figure 2 illustrates the general arrangement of the plant components. The total area within the fence is five acres. The three production wells are in the background with the brine pipeline terminating at the eight-unit brine/isobutane exchanger in the foreground. The two reinjection wells are outside the drawing to the right.

The cooling tower is $100 \mathrm{ft}$ wide and $200 \mathrm{ft}$ long. The condensing section is elevated in order to provide sufficient head to satisfy the NDSH requirements for the feed pumps.

The bullding to the left of the cooling tower houses the main turbogenerator unit. A 36 in. diameter Iine carries the turbine exhaust to the six regenerative heat exchangers between the building and cooling tower. The electrical switchgear yard is in the far left corner of the site. Across from the switchyard is a small water storage pond with a surface area of $7,200 \mathrm{ft}^{2}$ and a capacity of 270,000 gallons. The pond furnishes auxiliary

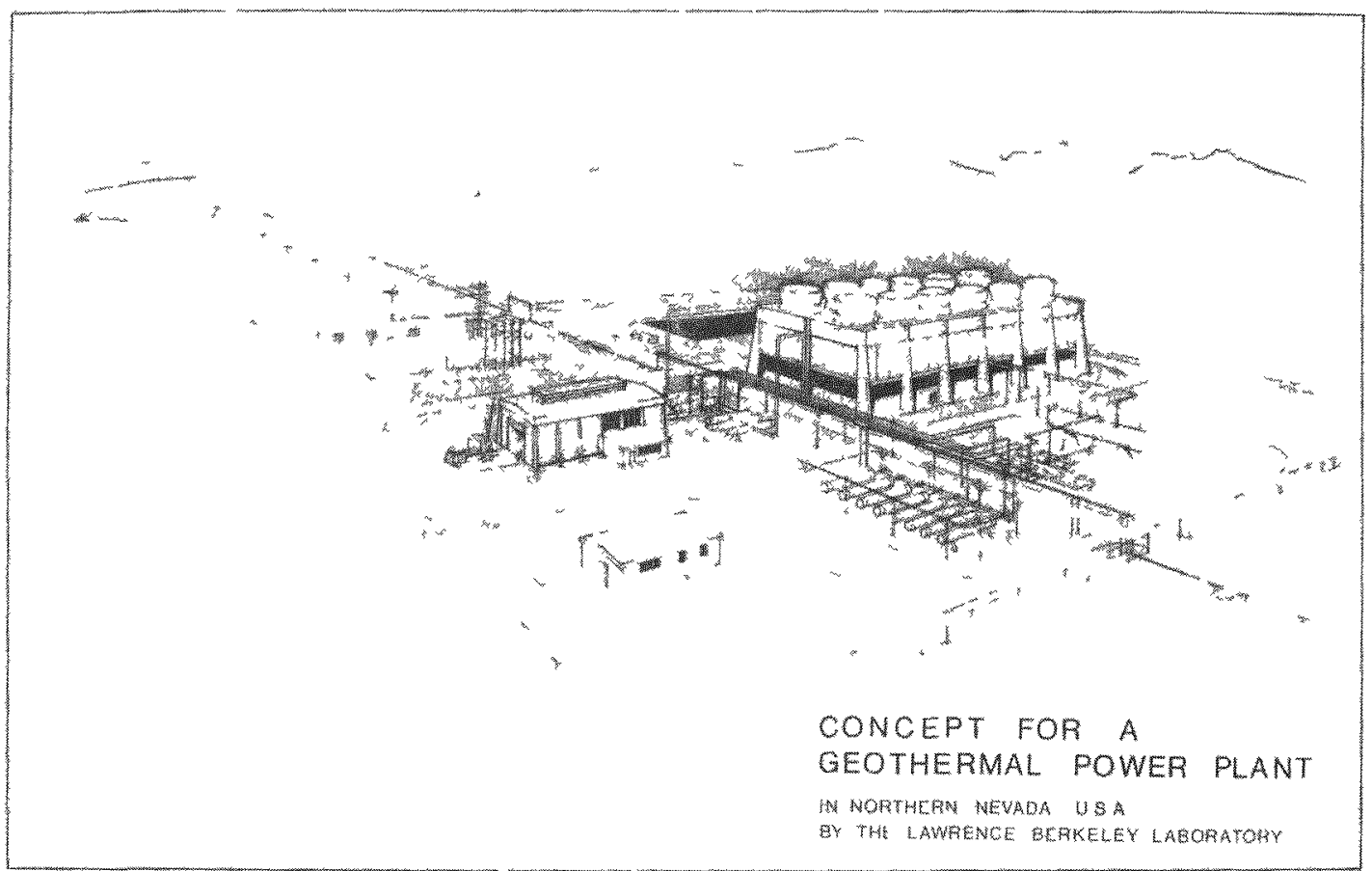

Fig. 2. Concept for a geothermal power plant. 
cooling for small heat loads and provides a source of water for fire protection.

A test pad where research and development work can be carried out under field conditions is adjacent to the substation. New binary fluids and prototype heat exchangers, turbines, condensers, pumps, etc., can be connected in parallel to plant components for realistic testing.

\section{REFERENCES}

1. D. L. Ray, "The Nation's Energy Future" (December 1973).

2. "Preliminary Conceptual Design of the $10 \mathrm{MN}$ Experimental Power Generation Facility", Lawrence Berkeley Laboratory, Jume 20, 1974.

\title{
THERMODYNAMIC PROCESS PROGRAM FOR GEOTHERMAL POWER PLANT CYCLES
}

\author{
M. A. Green, A. D. K. Laird, H. S. Pines
}

An important part of the geothermal program at the Lawrence Berkeley Laboratory is the design optimization of geothermal power plant cycles in general, and of binary cycles in particular. To accomplish this objective, a thermodynamic process program (GEOTHM ${ }^{1}$ ) has been developed and used to model some of the major geothermal power plant cycles. These include flashed steam, two-phase expander and bi-fluid binary cycles. The program assists in the design of a power plant through optimization of each component, given appropriate input data, and through cost calculations. It can also calculate the power output under off-design conditions.

\section{CALCULATION OF WORKING FLUID PROPERTIES}

The calculation of working fluid properties is the heart of program GEOTHM. Over half of the program is devoted to this process. Early in the development of the program it was decided that properties would be calculated using several equations of state rather than by interpolation of tabular data. The advantages of using the equation of state approach are as follows: (1) Tables require substantial memory and data handling becomes a problem; (2) Tables are often inaccurate and inconsistent; (3) Good tabular data is often not available near the critical point.

The working fluid properties are divided into four regions each with its own equation of state. Region 1 is the incompressible liquid region. Region 2 is the two-phase region inside the liquid vapor dome. Region 3 is the dense gas region on the gas side of the liquid vapor dome and the supercritical region above the dome. Region 4 is the ideal gas region. The equation of state in this region is applied to gases such as air, $\mathrm{CO}_{2}, \mathrm{SO}_{2}$ and $\mathrm{H}_{2} \mathrm{~S}$ when they are at room temperature and at atmospheric pressure or lower.

The equations of state for regions 1,2 and 4 are well defined and simple, and each is described by single computer subprogram. Region 3, however, presents particular difficulties because of the non-1inear nature of the fluid in this regime. Calculation of fluid properties in Region 3 requires eight major subprograms and an equal number of minor subprograms. Some of the calculations require several of the subroutines to be combined and then iterated by one of the major subprograms.
The program development has been slowed by convergence difficulties and the need to meld together several different equations of state which have differing properites.

Three equations of state are used to calculate the properties of 28 different working fluids. Two forms of the Martin equation of state 2,3 and the Starling equation of state 4 are built into the GEOTHM program. Al1 three equations of state have the property of approaching the ideal gas equation when the gas density approaches zero. At the critical point the equations of state have zero first and second derivatives of pressure with respect to density on the critical isotherm. The two forms of the Martin equation of state may be used with the following working fluids: $R-11, R-12, R-13$, $\mathrm{R}-21, \mathrm{R}-22, \mathrm{R}-23, \mathrm{R}-113, \mathrm{R}-114, \mathrm{R}-500, \mathrm{R}-502$, $\mathrm{C}-318, \mathrm{R}-717$ (ammonia), and R-600a (isobutane). (Note that the $\mathrm{R}$ number is the refrigerant designation by ASHRAE. 5 The DuPont trade name Freon may be substituted for $R$ in most cases.) The Starling equation of state, which is an extended version of the Benedict-Webb-Rubin equation, 6 is applied successfully to 1ight hydrocarbons. Program GEOTHM can use the Starling equation of state for the following working fluids: methane, ethane, propane (R-290), N-butane (R-600), isobutane (R-600a), $\mathrm{N}$-pentane, isopentane, $\mathrm{N}$-hexane, $\mathrm{N}$-heptane, $\mathrm{N}$-octane, ethylene, propylene, nitrogen (R-728), carbon dioxide $(\mathrm{R}-744)$, and hydrogen sulfide. Test cases using two equations of state for $\mathrm{R}-22$

(Freon-22 $\mathrm{CHClF}_{2}$ have yielded good agreement. Test cases have also been run with ammonia (R-717), isobutane $(\mathrm{R}-600 \mathrm{a})$, and propane $(\mathrm{R}-290)$. They have shown good agreement with ASHRAE data.

\section{THERMODYNAMIC PROCESS CALCULATIONS}

The simulation of a geothermal power plant requires the calculation of a number of thermodynamic processes. Each major plant component uses a separate subroutine to calculate its performance. These subroutines (there are fifteen of them) calculate the thermodynamics of such components as turbines, pumps, flash tanks, heat exchangers, contact condensers, dry condensers, cooling towe phase separators, and mixers. In addition, the effects of piping pressure drops and heat leaks may be simulated.

The most difficult process component to model is the heat exchangers. Counter flow heat exchangers 
are assumed in the calculation. With the appropriate coefficients of performance other types can be modeled as wel1. The program GEOTHM heat exchanger subroutine calculates state point temperatures, relative mass flows and area when the U factor, pinch point, $\Delta \mathrm{T}$ and the appropriate temperatures are given. The three basic types of heat exchangers which are modeled by the subroutine are found in geothermal power plant cycles and a number of other thermodynamic cycles.

Calculations for pumps, turbines, flash tanks, two-phase expanders, and contact condensers require very simple thermodynamic equations. The subroutines that do these calculations are short and are devoted mostly toward calculating the efficiency of the process being modeled.

The program performs a mass and energy balance on the thermodynamic cycle. The mass flow rates of the various fluids are set depending on whether the geothermal well flow is given or the net design power of the plant is given. In a geothermal power plant, the power plant efficiency and the energy yield per unit well flow (in $\mathrm{kWh}$ per metric ton) is calculated. The power plant data can be given in tabular form or it can be plotted. One of the plotting routines will make a $\mathrm{P}-\mathrm{V}$ diagram, a $\mathrm{T}-\mathrm{S}$ diagram, and a P-H (Molier) diagram of the power plant cycle. Computer plots have been made of energy yield per unit well flow as a function of various plant parameters.

\section{TEST THERMODYNAMIC CYCLES WHTH HAVE BEEN RUN BY GEOTHM}

A11 of the major geothermal energy conversion cycles have been run with program GEOTHM. Successful calculations have been made on flashed steam cycles, cycles using a two-phase expander on geothermal brines, and bi-fluid cycles (the so-called binary cycles). Most of the calculations have been applied to bi-fluid cycles using ammonia, isobutane, propane and Freon-22 as working fluids. Calculations have been done on combined cycles where several modes of energy conversion were used.

A typical geothermal power plant cycle will run in less than a second on the CDC-7600 computer. Short processing times are necessary for cost calculation, and the optimization of power plant design. Short processing times permit parameter studies of plant thermodynamic processes to be made. Such studies have been made on bi-fluid plants using ammonia and Freon-22 as working fluids. The program has reached the stage of development where thermodynamic comparisons can be made for various cycles which use a wide range of working fluids.

\section{REFERENCES}

1. M. A. Green and H. S. Pines, "Program GEOTHM, A Thermodynamic Process Program for Geothermal Power Plant Cycles", LBL-3060 (October 1974).

2. S. L. Milera, "Application of the Martin Equation of State to the Thermodynamic Properties of Ammonia", ORNL-TM-4413 (December 1973).

3. R. C. Downing, "Refrigerant Equations", DuPont Company, Freon Products Division, Report No. 2313.

4. K. E. Starling, "Fluid Thermodynamic Properties for Light Petroleum Systems" (Gulf Publishing Company, Houston 1973).

5. ASHRAE, Thermodynamic Properties of Refrigerants, 1969 American Society of Heating, Refrigerating and Air Conditioning Engineers.

6. M. Benedict, G. B. Webb, and J. Rubin, J. Chem. Phys. 8,334 (1940).

\title{
PREDICTING THE TEMPERATURE AT DEPTH OF NATURAL HOT AND COLD SPRINGS AND GEOTHERMAL WELL WATERS
}

\author{
A. J. Hebert and H. R. Bowman
}

Subsurface temperatures in geotherma1 reservoirs can be estimated by measurement of the silica concentration or sodium, potassium and calcium. ratios in the emerging waters. However, the significance of the measured temperatures is open to question, since both "geothermometers" depend on a number of conditions being met in the geothermal reservoir.

The quartz geothermometer is based on the known solubility of quartz in $\mathrm{H}_{2} \mathrm{O}$, and can be represented by the following equation:

$$
\mathrm{T}\left({ }^{\circ} \mathrm{C}\right) \mathrm{SiO}_{2}=-\frac{1647}{(4 / 3) \log \left(\mathrm{SiO}_{2}\right)+6.84}-273 \text {, }
$$

where $\mathrm{SiO}_{2}$ is in ppm. The predicted temperature will be high if steam separates as the water ascends. It may also vary if the hot waters are diluted, or mixed with waters in equilibrium with silicates other than quartz.
The $\mathrm{Na}, \mathrm{K}$, Ca geothermometer is based on the empirical relationship developed by Fournier and Truesde11 1

$$
\mathrm{T}(\mathrm{C}) \mathrm{Na}, \mathrm{K}, \mathrm{Ca}=\frac{1647}{\log (\mathrm{Na} / \mathrm{K}+\beta \log (\mathrm{Va} / \mathrm{Na})+2.24}-273,
$$

where $\mathrm{Na}, \mathrm{K}$ and $\mathrm{Ca}$ are concentrations in moles per $\mathrm{kg}$, and $\mathrm{B}=4 / 3$ for $\mathrm{T}<100^{\circ} \mathrm{C}$ and $1 / 3$ for $\mathrm{T}>100^{\circ} \mathrm{C}$. In this case, the predicted temperatures are not expected to vary as much with steam separation or dilution since they are dependent on ratios of concentrations.

To test the relative consistency of these two geothermometers, samples of hot spring and well waters were collected from sites in Nevada and California and analyzed by soft $x$-ray fluorescence techniques, and by neutron activation. 


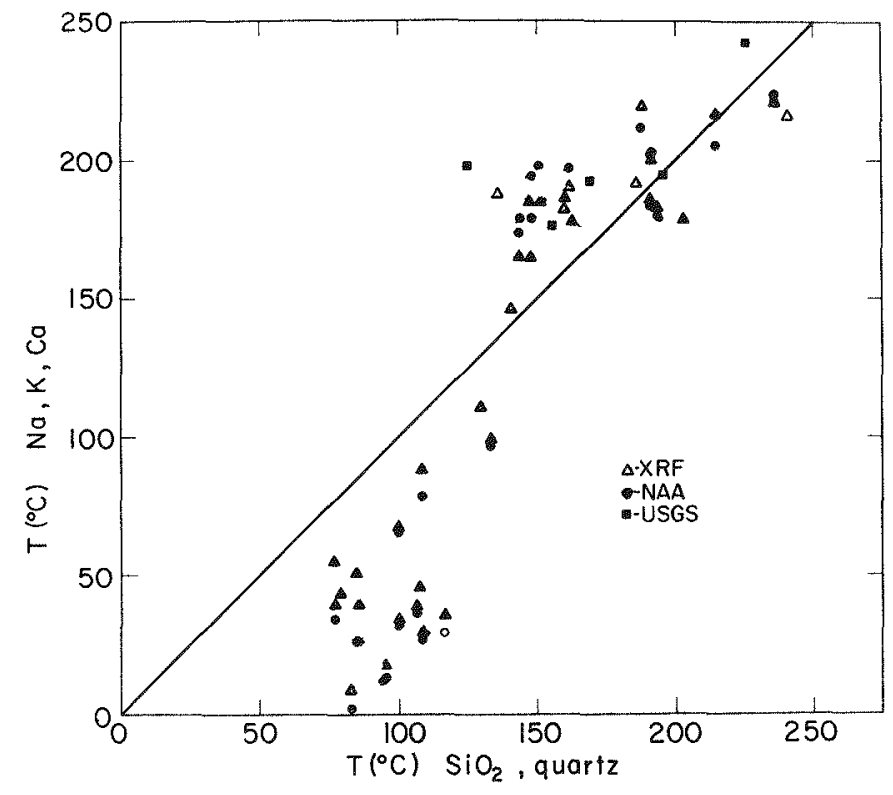

Fig. 1. A comparison between two methods for predicting temperatures of underground waters by chemical analyses of spring and well waters obtained at or near the surface. The "SiO 2 " or "quartz" geothermometer is based on the observed solubility of quartz in water. The "Na, $\mathrm{K}, \mathrm{Ca}$ " geothermometer is based on observed down-we11 temperature readings and chemical analyses for sodium, potassium, and calcium in the water.

(XBL, 748-3975)
The soft $x$-ray major element analyses were performed with $50 \mu 1$ samples in a manner previously described.2 Absorption corrections were made with a computer program and the values tabulated by McMaster, et a1.3

Figure 1 illustrates a comparison between the two methods of predicting temperatures. It is apparent that while the two geothermometers show reasonable agreement at temperatures above $125^{\circ} \mathrm{C}$, a large discrepancy develops at lower temperatures. At present, there is no suitable theory to explain the differences between the predicted temperatures below $125^{\circ} \mathrm{C}$. Further research should provide a clearer theoretical understanding of rock-water interactions and may lead to a more useful geothemometer.

\section{REFERENCES}

1. R. 0. Fournier and A. H. Truesde11, Geochim. Cosmochim Acta 37, 1255-1275 (1973).

2. A. J. Hebert, "Sulfur Determinations on Sma11 Biological Samples," Nuclear Chemistry Annual Report, January 1975, and in a paper presented at The $X$ Reunion Dela Sociedad Argentina De Investigacion Bioquimica entitled"Chemical Structure of Bungarotoxins" by V.A. Eterovic, L. W. Vickery, A. J. Hebert and E. L. Bennett, (1974).A. J. Hebert and Kenneth Street, Jr., Anal. Chem., 46, 203 (1974).

3. W. H. McMaster, et al., UCRL-50174 (May 1969), distributed by the National Technical Information Service, U. S. Department of Commerce, 5285 port Royal Road, Springfield, VA 22151.

\section{THERMODYNAMIC PROPERTIES OF HIGH TEMPERATURE BRINES}

K. S. Pitzer and L. F. Silvester

An important requirement for the development of liquid dominated geothermal systems is a satisfactory means of predicting the thermodynamic properties of high temperature brines or mixed electrolytes.

Our work on developing improved theories (or models) for dealing reliably and efficiently with the problems posed by such solutions has centered on evaluation of existing data for such thermodynamic parameters as (1) activity and osmotic coefficients, (2) heats of dilution and heats of solution, (3) heat capacities and (4) apparent molal volumes. The range of temperatures and pressures as well as the solutes will be chosen to meet the need for predicting the chemical and the engineering thermodynamic properties of geothermal brines.

The method of theoretical modeling involves extension of the basic equations developed by Pitzer. Starting with a general equation for the total excess Gibbs energy of a solution, the required derivatives were taken and appropriate functional forms introduced for the virial coefficients. For a simple salt MX the equations for the osmotic and activity coefficients, $\phi$ and $\gamma$, are

$$
\begin{aligned}
\phi-1= & -\frac{\left|z_{M} X\right| A_{\phi} I^{\frac{1}{2}}}{1+b I^{\frac{1}{2}}}+m\left(\frac{2 v_{M}{ }^{\nu} X}{\nu}\right)\left(\beta_{M X}(0)+\beta_{M X}^{(1)} e^{-\alpha I^{\frac{1}{2}}}\right) \\
& +m^{2}\left(\frac{2\left(\nu_{M}{ }^{\nu}\right)^{3 / 2}}{\nu}\right) c_{M X}^{\phi}
\end{aligned}
$$

and

$$
\begin{aligned}
\ln \gamma_{ \pm}= & -\left|Z_{M^{2} X}\right| A_{\phi}\left[\frac{I^{\frac{1}{2}}}{1+b I^{\frac{1}{2}}}+\frac{2}{b} \ln \left(1+b I^{\frac{1}{2}}\right)\right] \\
& +m\left(\frac{2 \nu_{M}{ }^{\nu} X}{\nu}\right)\left[2 \beta_{M X}^{(0)}+2 \frac{(1)}{M X}\left(1-\left(1+\alpha I^{\frac{1}{2}}\right.\right.\right. \\
& \left.\left.\left.-\frac{a^{2} I}{2}\right) e^{-\alpha I^{\frac{1}{2}}}\right)\right]+m^{2}\left(\frac{2\left(\nu_{M} X^{3}\right)^{3 / 2}}{\nu}\right)\left(\frac{3}{2} C_{M X}^{\phi}\right)
\end{aligned}
$$

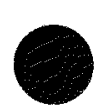


From Eqs. (1) and (2), one obtains a relationhip for the relative apparent molal heat content, in terms of the parameters $b, \alpha, \beta(0), \beta(1)$, and as

$$
\begin{aligned}
& \phi_{\mathrm{L}}=\frac{\nu}{2}\left|\mathrm{Z}_{\mathrm{M}^{2} \mathrm{Z}}\right| \frac{\mathrm{A}_{\mathrm{H}}}{3} \quad\left(\frac{2}{\mathrm{~b}}\right) \ln \left(1+\mathrm{bI}^{\frac{1}{2}}\right) \\
& -m\left(2 \mathrm{RT}^{2} \nu_{\mathrm{M}^{\nu} \mathrm{X}}\right)\left[\left(\frac{\partial \beta^{(0)}}{\partial \mathrm{T}}\right)_{\mathrm{p}}\right. \\
& +\frac{2}{\alpha^{2} I}\left(1-\left(1+\alpha I^{\frac{1}{2}}\right) e^{\left.-\alpha I^{\frac{1}{2}}\right)}\left(\frac{\partial \beta^{(1)}}{\partial \mathrm{T}}\right)_{\mathrm{p}}\right] \\
& \text { - } \mathrm{m}^{2} \mathrm{RT}^{2}\left(v_{M^{\nu}}\right)^{3 / 2}\left(\frac{\partial C^{\phi}}{\partial \mathrm{T}}\right) \text {. }
\end{aligned}
$$

Using Eq. (3), a computer program was developed and used to analyze data on heats of solution and/or heats of dilution for over one hundred different salts at room temperature. The results indicate that Eq. (3) reproduces the existing data substantially within experimental error with the parameters $b$ and $a$ taken as temperature independent. Results using Eqs. (1) and (2) for the available data on activity and osmotic coefficients of $\mathrm{NaCl}(\mathrm{aq})$ up to $300^{\circ} \mathrm{C}$ indicates the equations reproduce the data well within experimental error at the various temperatures with $b$ and a taken as temperature independent. These results are particularly satisfactory as they yield a compact set of equations whose parameters have physical meaning as far as possible.

There are relatively extensive experimental data on systems involving singly and doubly charged ions but few measurements on ions with higher charge. Hence, in addition to the theoretical efforts, an experimental program aimed at understanding the behavior of aqueous solutions containing high-charged species has been undertaken. Preliminary experiments indicate the salts $\mathrm{La}_{4}\left(\mathrm{Fe}(\mathrm{CN})_{6}\right)_{3}$ and $\mathrm{Ca}_{2} \mathrm{Fe}(\mathrm{CN})_{6}$ are suitable for cells similar to

$$
\mathrm{La}(\mathrm{ION})\left|\mathrm{La}_{4}\left(\mathrm{Fe}(\mathrm{CN})_{6}\right)_{3}(\mathrm{aq})\right| \mathrm{Ag}_{4} \mathrm{Fe}(\mathrm{CN})_{6}(\mathrm{~s}) \mid \mathrm{Ag}(\mathrm{s})
$$

where La(ION) is a lanthanum ion-specific electrode. The data from these cells will be analyzed in terms of "higher order" 1 imiting laws and to aid in picking appropriate theoretical models for dealing with electrolytes that undergo association in solution.

This program also receives some support through the Inorganic Materials Research Division of LBL.

\section{BACKGROUND STUDIES FOR PREDICTING SILICTC ACID BEHAVIOR IN GEOTHERMAL BRINES}

\section{A. R. Marsh, I. Huang, G. Klein, and T. Vermeulen}

Amorphous deposits of silica and silicates are foremost among several types of equipment fouling that occur in power generation from geothermal brines. In order to understand the mechanism and rates of silica fouling in geothermal brines, studies of silicic acid polymerization have been made at $25^{\circ} \mathrm{C}$ on solutions in the range of 100 to $1000 \mathrm{ppm}$ dissolved silica $(0.0015 \mathrm{M}$ to $0.015 \mathrm{M}$ $\mathrm{SiO}_{2}$ ) at $\mathrm{pH}$ values between 4 and 10 .

The chemical structure of silicic acid in its various forms is illustrated by three examples:<smiles>O[Si](O)(O)O</smiles><smiles>O[Si](O)(O)O[Si](O)(O)O[Si](O)(O)O</smiles>

\section{Cyclic Trimer}

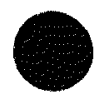<smiles>O[Si]1(O)O[Si](O)(O)O[Si](O)(O)O1</smiles>

Silicon atoms, like aliphatic carbon, appear to form only tetrahedral bonds. The linear polymers slowly "age" by further dehydration to monocyclic and polycyclic forms. Quartz, the infinite-polymer crystal, is completely crosslinked, containing no unreacted -OH groups except at the crystal surface. In this study no evidence has been found for the presence of any silicycl groups $=\mathrm{Si}=0$. At higher $\mathrm{pH}$, the $-\mathrm{OH}$ groups can ionize to $-\mathrm{C}^{-}$ and $\mathrm{H}^{+}$. The $\mathrm{pK}$ for monomer is near 10.0 and for dimer neax 9.7 , for the first hydrogen to ionize. A higher ionization constant, or lower $\mathrm{pK}$, is inferred for $-\mathrm{OH}$ attached to $-\mathrm{O}-\mathrm{S}_{\mathrm{i}}^{\mathrm{i}} \mathrm{-O}-\mathrm{O}$, which arises in the trimer and higher-polymer forms, indicating that these polymers are stronger acids than the monomer or dimer.

Polymers form in increasing amounts at progressively higher levels of total dissolved silica. The monomer concentration itself always remains below $0.0020 \mathrm{M}$ which corresponds to the amorphous silica solubility reported by Morey, Fournier, and Rowe ${ }^{1}$ and Alexander, Heston, and Iler. 2 Silica is believed to dissolve in water only as monomeric silicic acid.

Many previous investigators have studied the behavior of silicic acid in concentrated solutions, that is, above $6000 \mathrm{ppm}$ or $0.10 \mathrm{M}$. The present study has established definite behavioral similarities between dilute and concentrated solutions, and thus clarifies the rate behavior and equilibrium effects in polymerization. Figure 1, from published work, 3 shows the time required to reach a gel condition in concentrated solutions, and emphasizes the strong effects of $\mathrm{pH}$ and total concentration. 


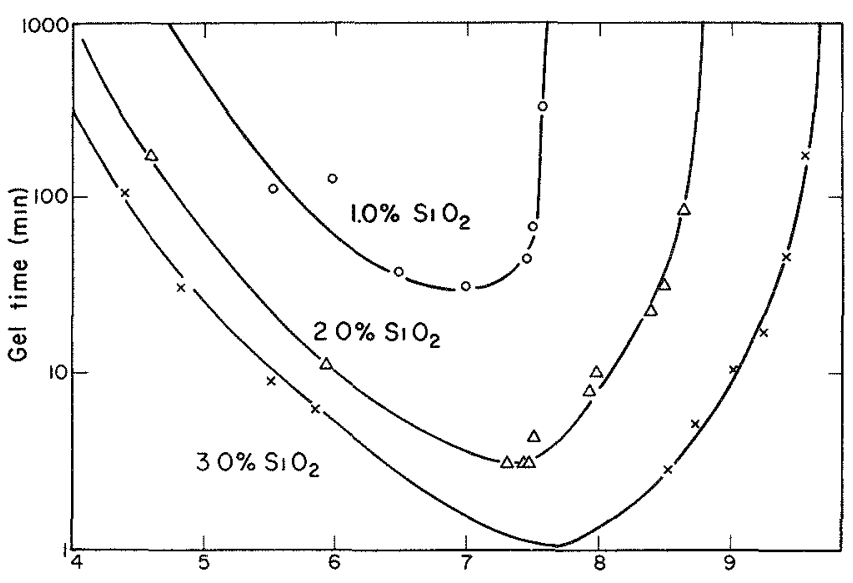

Fig. 1. Gel time vs. pH at $25^{\circ} \mathrm{C}$, after Merrill and Spencer. 3

(XBL 761-2181)

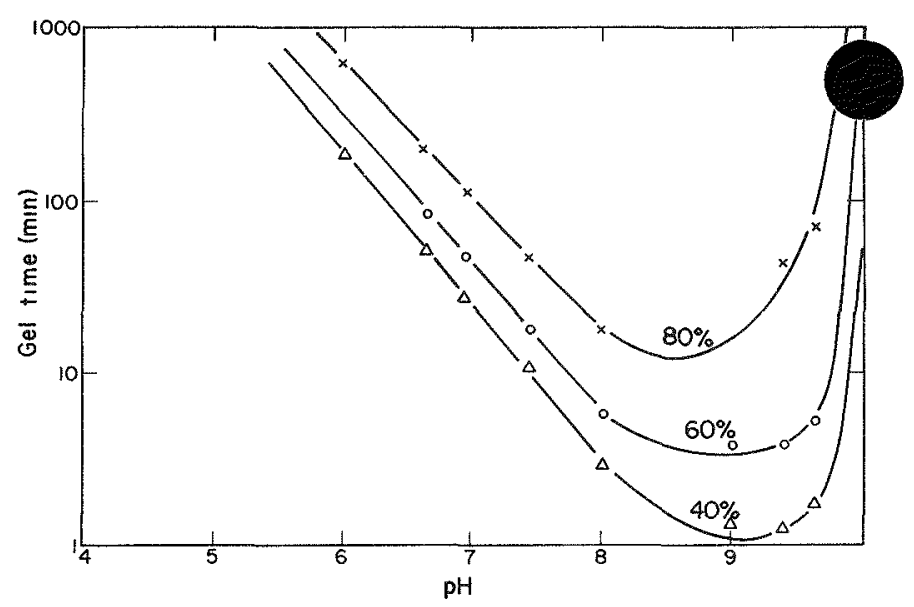

Fig. 2. Fractional approach to monomer equilibrium vs $\mathrm{pH}$ at $25^{\circ} \mathrm{C}$. Average concentration (as $\mathrm{SiO}_{2}$ ) is 870 ppm.

(XBL 76I-2180)

average polymerization rate for progressively higher conversion has its maximum at progressively lower pH values.

Polymerization rates of silicic acid in dilute solution have long been known to be second-order in silicic acid itself and first-order in hydroxide ion. 4 This indicates reaction between a neutral molecule and a silicate anion, and suggests that the maximum rate should occur close to $\mathrm{pH}$ 10. However, the bulk reaction shows strong autocatalytic effects, as well as a shift in $\mathrm{pH}$ for the maximum rate. It is found that polymerization in unbuffered solutions leads to $\mathrm{pH}$ increases in the dilute range, and to $\mathrm{pH}$ decreases in the more concentrated range. All these results are consistent with the view, expressed above, that trimers and higher polymers are stronger acids than monomer and dimer.

Studies on silica polymerization will be extended to include the effects of higher temperature and of cationic and anionic co-reactants.

\section{REFERENCES}

1. G. W. Morey, R. O. Fournier, and J. J. Rowe, J. Geophys. Res. 69, 1995 (1964).

2. G. B. Alexander, $\vec{W} . M$. Heston, and R. K. I1er, J. Phys. Chem. 58, 453 (1954).

3. R. C. Merril1, and R. $\bar{W}$. Spencer, J. Phys. S. Colloid Chem. 54, 806 (1950).

4. A. D. Bishop and J. I. Bear, Thermochim. Acta 3, 399 (1972). 
MEASUREMENT OF TIE PROPERTTES AND BEHAVTOR OF ROCK-FLUID SYSTEMS AT ELEVATED PRESSURES AND TEMPERATURES

\section{W. H. Somerton}

Rock properties have been measured at stress conditions simulating great depths but little work has been done at the unusually high tempertures encountered in geothemal reservoirs. Techniques for measuring the physical properties of rocks at simulated geothermal reservoir environmental conditions are being developed in the present work. The properties to be measured include bulk and matrix compressibilities, dilatational and shear velocities, thermal conductivity and diffusivity, thermal expansion, permeability and formation resistivity factors. The range of envirommental conditions include temperatures to $400^{\circ} \mathrm{C}$, confining pressures to 20,000 psi, fluid pore pressures to half that amount and saturations ranging from vacuum to full brine saturation.

In developing measuring techniques using existing pressure cells, temperatures were limited to $200^{\circ} \mathrm{C}$ and pressures to $15,000 \mathrm{psi}$, but by developing an in-situ saturation - desaturation technique, any desired condition of fluid saturation could be obtained. These techniques and the results obtained are reviewed briefly. Progress and problems associated with the development of a composite rock properties test apparatus which will meet the desired temperature and pressure conditions are a1so discussed.

The compressibility measuring component of the present apparatus employs a sensitive deflection sensor to measure change in length of a cylindrical core sample as pressure is changed. Thermal expansion can also be evaluated as temperature is changed. For isotropic samples volume compressibility is taken as three times the Iinear contraction. For anisotropic samples, linear contractions in directions parallel and perpendicular to the bedding planes must be measured. Bulk compressibility is measured on jacketed samples and matrix or grain solids compressibility on unjacketed samples of the rock. Details of the apparatus and measuring technique are given by EI Shaarani.1

Results of compressibility measurements are given by E1 Shaarani 1 and are reviewed by Somerton. 2 These results show that in general, bulk compressibility decreases with increased pressure but increases with increased temperature and with increased liquid saturation. Matrix compressibility changes only with temperature, increasing with increased temperature. In applying these results, E1 Shaarani shows that land surface subsidence due to subsurface fluid withdrawal will be greater in geothermal areas because of the elevated tenperatures.

Dilatational and shear velocities of rocks are measured by the first pulse arrival technique using lead zirconate titanate piezoelectric crystals for pulsing and detection of the sonic waves. The apparatus and techniques employed in these measurements are described by Mobarak ${ }^{3}$ and $A 1$ Khafaji. 4 Results show that both dilatational and shear velocities increase with increased pressure and decrease with increased temperature. A substantial. difference is noted in the effect of liquid saturation - dilatational velocity increases with increased 1iquid saturation whereas shear velocity decreases. This latter difference leads to the possibility proposed by Al Khafaji 4 that liquid levels or liquid-gas (vapor) contacts could be detected by this difference in behavior. Mobarak ${ }^{3}$ has applied the temperature effect on dilatational velocity in interpreting sonic logs. He shows that temperature corrections need to be made to avoid error in the usual sonic transit time-porosity correlation. There is nomally a direct linear relation between transit time (reciprocal of dilatational velocity) and porosity. However, the effect of temperature is to increase transit time and to decrease porosity. This opposite effect could lead to porosity errors of $20 \%$ or more in high temperature reservoirs.

Thermal conductivity and diffusivity are measured by a transient technique described by Nguyen. 5 The method involves the application of heat at a constant rate to a small area of an extensive rock surface. The mathematical solution for this problem is available $e^{6}$ in terms of dimensionless temperature rise and dimensionless time. Comparing the actual temperature-time history with the dimensionless solution, Nguyen used a curve matching technique which gives both thermal conductivity and diffusivity.

A steady state method is also used to measure thermal conductivity and to evaluate the vaporization-condensation-capillary (or so-called "heatpipe") effect. This latter effect, discussed by Somerton, 2 occurs when a partially liquid saturated rock at or near its vapor pressure has a temperature differential imposed across it. Heat is transferred not only by conduction but also by the latent heat of vaporization-condensation. The continuous fluid flow cycle is completed by capillary flow of condensed 1iquid to the vaporizing section of the system. A lumped parameter is used to evaluate the magnitude of the VCC effect.

Results of thermal properties tests show that both thermal conductivity and diffusivity increase slightly with increased effective stress. These two properties usually decrease in magnitude with increased temperature but for some low thermal. conductivity systems, the opposite might be true, values increasing with increased temperature. The magnitude of the VCC effect is related to relative permeability in that for a given system it reaches a maximum in the middle range of 1 iquid saturations. The magnitude of the peak value is strongly related to the absolute permeability of the rock.

The application of the compressibility measuring system to thermal expansion measurements has not yet been tested. Thermal expansion data have been obtained by use of a separate apparatus 8 which has no provisions for stress application or liquid saturation. Measurements with this device show that thermal expansion coefficients generally increase with increasing temperature up to the $\alpha-\beta$ 
quartz inversion temperature $\left(575^{\circ} \mathrm{C}\right)$ beyond which temperature the coefficient is greatly decreased and may become negative for high quartz content rocks.

The permeability measuring device is provided with a back pressure regulator so that the pore pressure is maintained above vapor pressure and the flowing fluid is maintained in the liquid phase at the elevated temperatures. Preliminary results show that liquid permeabilities of Berea and Boise sandstones decrease substantially upon increase in temperature. Others 7 have observed this effect but have also noted that permeability to gases does not vary with temperature. S1ight modifications will be made in the present apparatus so that the vapor phase (steam) only will flow through the core. It will thus be possible to compare liquid and vapor permeabilities to determine whether the temperature effect persists.

Provisions for measuring formation resistivity factors have been added to the permeability measuring apparatus. The four-electrode resistivity measuring device is made up of four silver plated wires wrapped around the core before sleeving the two wires near the ends of the cores serving as current electrodes and the two inner wires being potential measuring electrodes. By coupling with the pore fluid, these wire rings act as disc electrodes. This resistivity measurement in no way interferes with the permeability measurements. Tests are now in progress to compare results obtained by this technique with measurements made by conventional methods.

Formation resistivity factors have been found to increase with increased temperature. ${ }^{7}$ Linking Archie's equation for the formation resistivity factor with the Kozeny-Carmen equation for permeability, an inverse relation between the two quantities would be expected. The common linking factor is tortuosity which apparently is quite sensitive to changes in temperature. Investigation of this matter is in progress.

Design of the new high temperature physical properties measuring apparatus has been started, and the pressure ce11 drawings and specifications have been sent out for bid. The objective is to measure all of the desired properties with a single apparatus under identical conditions so that meaningful correlations may be obtained. This presents some problems because of conflicting requirements for some of the tests. Most of these problems have be worked out and the major problems remaining are those of bringing the many electrical leads out of the cel1, a satisfactory high-temperature jacketing material for the core, and development of a high temperature printed-circuit system for the therma1 conductivity disc heater, the thermocuples and the resistivity measurement electrodes. Electrical insulation in a brine environment at high temperature appears to be a major problen. It is planned to complete the design and construction of the apparatus using the best methods and materials available even if this means that the first tests will. be restricted to temperatures somewhat below those ultimately planned.

\section{REFERENCES}

1. A. H. El Shaarani, "Deformation Moduli of Water Bearing Formations at Elevated Temperatures," M.S. Research Report, Univ. of Calif., Berkeley (Dec. 1973).

2. W. H. Somerton, "Deformation Moduli of Water Bearing Formations at Elevated Temperatures," Calif. Water Resources Center, Contrib. No. $146(1974)$.

3. S. Mobarek, "The Effect of Temperature on Wave Velocities in Rocks," M.S. Thesis, Univ. of California., Berkeley (Sept. 1971).

4. A. A1 Khafaji, "Effects of Pressure, Temperature and Liquid Saturation on Shear Velocities in Rocks," M. S. Thesis, Univ . of Calif., Berkeley (Dec. 1974).

5. T. C. Nguyen, "Transient Measurement of Therma1 Properties of Rocks under High Pressures and Temperatures," M. S. Research Report, Univ . of Calif., Berkeley (June 1974).

6. M. A. Selim, I. Fatt, and W. H. Somerton, "Temperature Rise in a Semi-Infinite Medium Heated by a Disc Source," proceedings, Series A, 66, No. 4, Koninki. Neder1, Alkademie Van Wentenschappen, Amsterdam (1963).

7. S. K. Sanyal, S. S. Marsden, and J. H. Ramey, "Effect of Temperature on Petrophysical Properties of Rocks," Soc. of Pet Engrs. Preprint 4898 (Oct. 1974).

8. W. H. Somerton, and M. A. Selim, "Additional Thermal Data for Porous Rocks - - Thermal Expansion and Heat of Reaction," Soc. Pet. Engrs. J., Vol. 2, No. 4, p. 249 (1961).

\section{GEOTHERMAL RESERVOIR DYNAMICS}

\section{P. A. Witherspoon and V. E. Schrock}

The major effort in the research program on geothermal reservoir dynamics has been on the problem of developing a computational capability of mathematically modeling the behavior of geothermal systems. In earlier work on the University of California campus, a three-dimensional code was developed that can handle single-phase flow of fluids and the transport of heat through porous media. This single-phase program has been used to model the steady-state behavior of liquid-dominated systems. Two- and three-dimensional reservoirs have been modeled satisfactorily using systems consisting of one to three layers. In this initial effort, we have been mainly interested in examining the heat flow and the convection cells that can form for simple geometries where the Rayleig number within the "reservoir" of a multi-layer system is kept constant.

It is apparent from the rather simple systems that have been studied that the geological constraints play a very important role in controlling 
the size of the convecting cells and thus the total heat flow through the system. Figure 1 shows an example of the type of results that are obtained for a three-layer model where Layer A is sufficiently porous and permeable to sustain the development of four convection cells. Layer A has a Rayleigh number of 100 and is enclosed by impermeable Layers $B$ above and below, where only heat conduction takes place (i.e., $\mathrm{Ra}=0$ ). The Nusselt number for this particular model is 1.2 , meaning that heat transfer through the system is only $20 \%$ greater than would be obtained by conduction alone throughout the whole system. The thermal properties for each layer were identical, and the finite hydraulic conductivity assigned to Layer A permits the development of the convection cells.

We have also begun the development of an ana$\log$ model using an experimental sand box that was

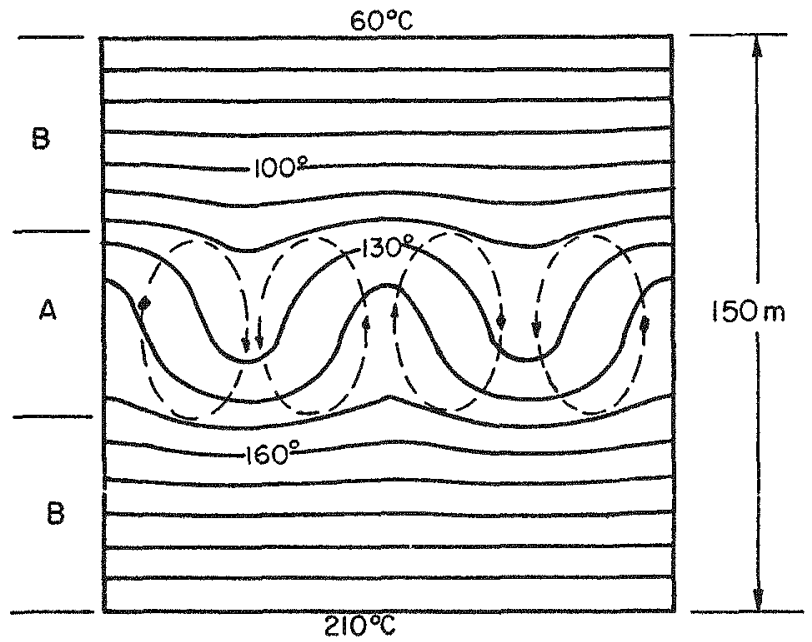

Fig. 1. Development of convection cells in threelayer system. $\mathrm{Ra}=100$ in Layer $\mathrm{A} ; \mathrm{Ra}=0$ in Layers B.

(XBL 751-2007) constructed in earlier research on the University of California campus. This box has dimensions of $0.9 \times 1.2 \times 1.8 \mathrm{~m}$ and when properly packed with sand will have a permeability in the range of 10-12 to $10^{-10} \mathrm{~m}^{2}$ (1 to 100 darcies) depending on the grain size distribution that is selected. We are now instrumenting the box with appropriate insulation and heat sources as well as standard devices for temperature and pressure measurements. We wish to study the utility of such a laboratory approach in providing models of both natural and forced heat convection systems in porous media. This can give us a means of checking the accuracy of our mathematical models and should also provide an insight into some of the mechanisms of geothermal reservoir behavior.

Ana1ytical work has led to a paper, "Theory of Heat Extraction from Fractured Hot Dry Rock," by A. C. Gringarten, ${ }^{*}$ P. A. Withersppon, and Y. Ohnishi,t which has been accepted for publication in the Journal of Geophysical Research in 1975. This paper presents a theory for the amount of heat that can be extracted from an infinite series of parallel vertical fractures of uniform aperture. The fractures are uniformly spaced and drain heat from blocks of homogeneous and isotropic impermeable rock. Cold water enters at the bottom of each fracture and solutions are given in terms of dimensionless parameters from which the exiting water temperatures at the top of the fractures can be determined. Application of the theory to a field situation demonstrates how a multiple-fractured system provides a more efficient mechanism for heat extraction than a single fracture in hot dry rock.

\section{Footnotes}

*Present address: Bureau Recherches Geologiques et Mineres, Service Geologique National, Orleans, France.

†Present address: Department of Civil Engineering, Kyoto University, Kyoto, Japan

\section{NATIONAL GEOTHERMAL INFORMATION RESOURCE (GRID)}

F. B. Henderson III, S. L. Phillips, and T. Trippe

A Geothermal Data Center is being established at the Lawrence Berkeley Laboratory as a joint effort between the Energy and Enviroment Division and the U. S. Geological Survey, with the objective to collect, store, and disseminate information on geothermal science and technology. The center, known as the National Geothermal Information Resource, is often called by the Acronym GRID (Geothermal Resources Information and Data). Both bibliographic and numerical data are being compiled using the following two formats:

1) a loose-leaf handbook, and 2) a computer-based information storage and retrieval system. The reults of the compilation will include an annotated and indexed bibliography of geothermal literature and data, critical comparisons among alternative methods of geothermal exploration and exploitation, and recommendations for improvements in areas requiring more or better data collection.
The activities of the center are overseen by a Technical Advisory Committee whose charge includes establishing priorities and reviewing progress. The current members of the committee are as follows:

L. O. Beaulaurier Bechtel Corporation San Francisco, CA

C. W. Berge Phillips Petroleum Company Del Mar, CA

Barry Block Diax Corporation Sumnyvale, CA

Richard G. Bowen State of Oregon Portland, $O R$ 
Merrill Cohen

Genera1 Electric Company

Lynn, MA

Robert 0. Fournier

United States

Geological Survey

Menlo Park, CA

Thomas C. Hinrichs

San Diego Gas and

Electric Company

San Diego, CA

John H. Howard

Lawrence Livermore Laboratory

Livermore, CA

Ronald L. Kreiling

$E G \& G$

San Ramon, CA

William C. Klostermeyer

Bureau of Reclamation

Washington, D. C.

J. J. Patrick Muffler

United States

Geological Survey

Menlo Park, CA

Morris S. Phillips

U. S. Corps of Engineers

Wal tham, MA

Morton C. Smith

Los Alamos Scientific

Laboratory

Los Alamos, New Mexico

Paul A. Witherspoon

Lawrence Berkeley Laboratory

Berkeley, CA

The activities at LBL are coordinated with other activities that relate to the collection and dissemination of geothermal information and data. Currently, the principal interactions are those with the U. S. Geological Survey (USGS), the NATO Committee on Challenges of Modern Society COMS, and the Energy Data Base being established at the Technical Information Center (TIC) in Oak Ridge by the AEC's office of Scientific Information. The LBL coordination with the U. S. Geological Survey is primarily with their Office of Resource Analysis in Reston, Virginia. The USGS is interested in acquiring and compiling data that pertain to geothermal fields/areas and flowing wells. The coordination with NATO/CCMS is aimed primarily at international exchange of geothermal information between interested nations such as Iceland, New Zealand, Italy, West Gemany, France, Mexico, and the United States. There are four principal data centers involved; these are: LBL, the U. S. Geological Survey in Reston, Virginia, the Geothermal Research Institute in Pisa, Italy, and the Department of Scientific and Industrial Research in We11ington, New Zealand. In the coordination with the TIC we are jointly developing a thesaurus for geothermal energy, and are exchanging data to ensure comprehensive coverage of the geothermal literature.
The myriad facets of geothermal information can be categorized in a number of ways. We have fc it convenient to classify the world wide geother information into the following four principal categories:

1. Exploration and Evaluation (e.g., drilling, economic evaluation, fields, geologic and hydrologic surveys, geophysics, geochemistry, isotope chemistry, research and advanced technology, U, S, and world resources development, volcanology, flowing wel1s).

2. Physicochemical Properties (e.g., densities and vapors pressures, reservoir minerals and rocks, thermodynamic and thermophysical data at elevated temperatures and pressures, synthesis of pure geothermal substances, rock-solutiongas kinetics, volcanology, working fluids).

3. Utilization (e.g., binary systems power generation, corrosion and scaling, economic evaluation, electric power production, space and process heat, fluid reinjection, steam and hot water transmission, working fluids).

4. Environmental and Institutional Considerations (e.g., gaseous emissions, regulatory constraints, subsidence, noise).

Besides abstracts of the geothermal 1iterature, GRID will provide the user a compilation of background information concerning the exploration and exploitation of geothermal energy. For example, the compilation will include a narrative overview portion covering topics such as: categories of geothermal resource systems (e.g., vapor-dominated, 1iquid-dominated, hot-dry-rock, magma pools, and geopressure); economic and social aspects of the exploration and exploitation of the nation's geothermal resource; organizations that are involved in geothermal energy development; tabulation of the units used in the geothermal area; and a glossary of terms.

The compilation of geothermal information and data will be available for dissemination in two basic formats. User oriented computer aided recall programs will be in bibliographic (abstract) output formats or numerical data output formats. The information and data currently in the GRID System will be immediately available through these formats at user's request.

The second main type of output will be the loose-1eaf handbook entitled Compilation of Geothermal Information that will be published in four volumes corresponding to the four main categories of geothermal information. These volumes will be issued to subscribers in loose-1eaf sections as they become available. The user may also subscribe to the handbook updating service. This service will provide the user with periodic additions of current abstracts as we11 as narrative section updatings and re-writes as required by advance in the state of knowledge concerning thermal energy.

The availability of the four volumes of the Compilation of Geothermal Information is programmed for completion as follows: 
Volume 1. Exploration and Evaluation-.year end 1975;

Volume 2. Physical Chemistry---mid 1976;

Volume 3. Utilization---early 1977; and

Volume 4. Environmenta1, Lega1, and Institutional Consideration---year end 1977.

These volumes will be issued by sections as ready. For example, the Introduction, Geochemistry, and Drilling sections of Volume 1, Exploration and Evaluation will be available by May 1975, and the remaining sections will be distributed by year end.

To reach maximum effectiveness as a useroriented geothermal information and data compilation and dissemination system, GRID's projected 5-year program and 1980 goal are as

\section{Milestones, FY1975-FY1980}

FY75: Demonstration File Socorro Report Indexing

Initial Storage and Retrieval Program

Preliminary Thesaurus of Geothermal Descriptors

FY76-FY80: Complete "Catch-up" Phase Information Storage and Retrieval Program
Data Tagging

Data Quality

Computer Links

Package Searches

Direct Computer Searches

Profile Searches

Special Restrospective Searches

Leases and Exchanges of GRID

Tapes

Goa1, FY1980

GRID will be a ready source of interpreted and evaluated data compiled from the published 1iterature of the world, and will provide a variety of services in the transfer of this information from the originator to the ultimate user.

\section{Acknowledgement}

We acknowledge the generous assistance and valuable advice of C. M. Lederer, D. R. Richards, V. White, G. S. Haire, J. J. Herr, K. M. Mirk, H. A. WoIlenberg, and D. A. Mack, a1l of the Lawrence Berkeley Laboratory. We are grateful to the Information Research Group for their work on development of the Geothermal Thesaurus; to the Particle Data Group for development of the data base management system (DBMS); and, to the Table of Isotopes Group for their generous overall assistance. 


\section{Plasma Physics and Controlled Thermonuclear Fusion Research (CTR)}

INTRODUCTION

Plasma Physics and Controlled Fusion Research has been a continuing activity at the Lawrence Berkeley Laboratory since 1955, when it was still classified under the name Project Sherwood. Until 1973 this program was administered entirely through $M$ Division at the Lawrence Livermore Laboratory (LLL) which had been designated one of the four major sites for CTR work for the USAEC. Thus a11 annual reports on this activity up to and including fiscal year 1973 appear as a part of those issued by LLL.

Starting with fiscal year 1974, the portion of the CTR program at LBL dealing with basic research on plasma physics, plasma production and heating, and related atomic physics, was separated out, henceforth to be administered directly by the appropriate Division within the laboratory at Berkeley. The project on neutral-beam development for injection into fusion devices continues as a joint effort administered by LLL.

When the Energy and Environment Division was established it incorporated the CTR program at LBL. Thus progress and status of the LBL plasma physics and fusion-related research will henceforth be covered in the annual reports of the Energy and Environment Division.
The CTR Project at LBL consists of the following five major parts:

1. Experimental research on plasma production and trapping of laser-ionized pure deuterium pellet plasma in a magnetic field.

2. Atomic physics studies and cross section measurements, most1y motivated by the neutral-injection problem.

3. Heating and confinement studies in the "Tormac" configuration. This is a new project at $\mathrm{LBL}$, recently transferred from the Air Force Cambridge Research Laboratory, and considerably expanded through a contract with the Electric Power Research Institute.

4. Neutral beam development. This joint $\mathrm{LLL}_{2} / \mathrm{LBL}$ program is developing hardware for neutral beam injectors.

5. Theoretical research, mainly dealing with plasma heating by nonlinear interaction of waves with the medium.

PLASMA PRODUCTION AND TRAPPING OF LASER-IONIZED PURE DEUTERTUMPELLET PLASMA IN A MAGNETIC FIELD

N.E. Abt, T.R. Jarboe, * W.B. Kunke1, A.F. Lietzke, and G.H. Rankin

\section{INTRODUCTION}

The original purpose of this so-called "laserplasma" experiment at LBL was the reliable production of a completely isolated and pure hydrogen or deuterium plasma in the energy range of $100 \mathrm{eV} /$ particle, and its capture in a magnetic field, for use in a variety of basic studies. Preparation for this project and the development of the essential components took several years. Thus major parts and preliminary results have been described in previous annual reports ${ }^{1}$ and only a brief summary is given here.

In the meantime, knowledge of the behavior of plasma produced by laser irradiation of a levitated or freely falling solid pellet and its expansion in a magnetic field has gained new significance because of the possible use of such plasma as target material in Basebal1 II (at LLL) as we11 as in the United Aircraft mirror-confinement experiments. Therefore this particular study is now primarily intended to supplement those at the United Technologies Research Center with a diversity of diagnostic tools and by substituting pure deuterium for the usual lithium hydride.

\section{EXPERIMENTAL APPARATUS}

The apparatus used in this experiment consists of the following components: the pellet producer, a giant-pulse ruby laser system, pellet detection and laser firing systems, vacuum chamber and magentic field production equipment, and plasma diagnostic devices. The basic layout is shown in Fig. 1 .

Pellets are produced, collimated, and allowed to fall freely in a uniform magnetic field toward the focal region of the ruby laser pulse. Most of the pellets do not fall through this focal region, but when a pellet is on the right trajectory to fall through the focus region, the detection system triggers the ruby laser in such a way that the laser fires when the pellet reaches the focus. After the pellet is irradiated, the plasma produced expands in the uniform magnetic field (which can be set equal to zero) and is studied with collectors, magnetic probes, and a "plasma camera". 2

In the pellet maker, described more completely elsewhere, 3 the small pieces are produced by slicing a $50-u m$ diameter thread into approximately 


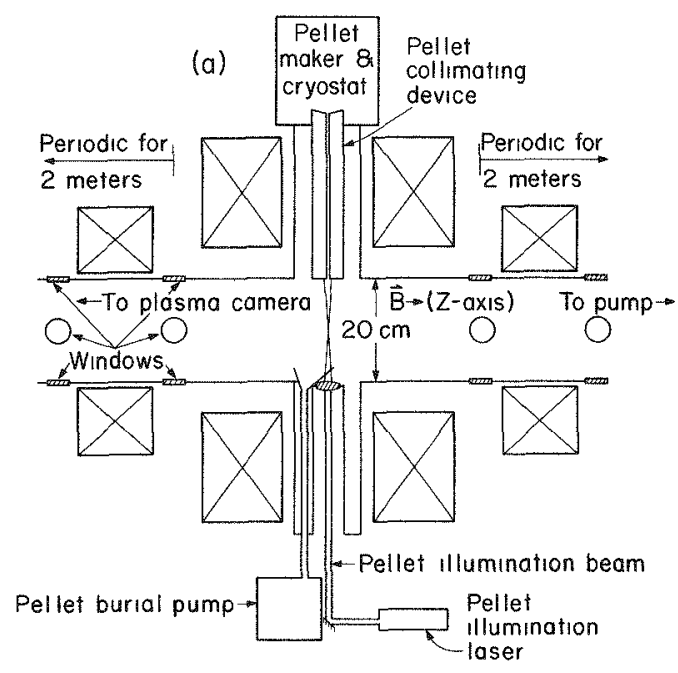

Side view

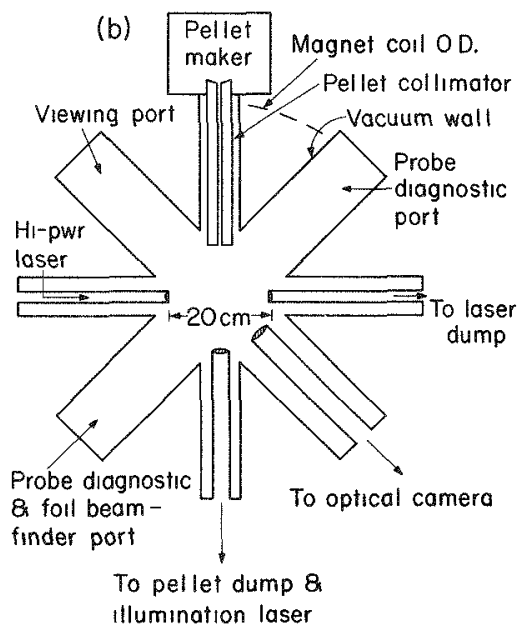

End-on view

Fig. 1. Principal components of the laser-ionized pellet experiment. The high-power laser is focused on the so-called "zap-zone", on the axis of the magnet solenoid.

(XBL 749-4248)

50-um length cylinders with a thin tungsten wire. The solid thread is made by allowing liquid deuterium of the proper temperature and pressure to flow through an aperture into a vacuum. The temperature is controlled through the vapor pressure of continously produced liquid hydrogen coolant. The pellets are collected by a funnel and collimated by falling through a quartz capillary tube carefully aimed at the focal spot of the ruby laser.

The remainder of the apparatus is rather conventional, and is shown in Fig. 1. A complete description of the experiment is given in a recent Ph.D. thesis. 4 The results obtained to-date are summarized below

\section{FORMATION PROCESS OF DEUTERIUM PLASMA CLOUDS}

The freely falling solid pure deuterium pellets of about 50- $\mu \mathrm{m}$ diameter are converted into plasma of about $100 \mathrm{eV} /$ particle energy if a fast rising (1-nsec risetime) 2-joule ruby laser pulse is used. Comparison with simplified computational models shows that the observed she11-1ike and not quite symmetric plasma clouds are consistent with the following process: Light absorption causes high pressure and ablation both backward and sideward. This pressure drives a strong shock through the pellet converting it into a forward-moving flattened partially ionized superdense "fluid". When the shock arrives at the far end of the pe1let, the material there blows off in a free expanon resulting in a retrograde rarefaction wave. ter this rarefaction front reaches the ablation surface the latter accelerates as the density ahead decreases. The plasma cloud thus emanates effectively from an accelerating source region. The process teminates fairly abruptly after the absorption length becomes comparable to the thickness of the remaining compressed material.

\section{EARLY STAGES OF EXPANSION OF THE PLASMA CLOUDS IN} A MAGNETIC FIELD

Observations with a set of charge detectors, magnetic-flux loops and a "plasma camera"2 on the evolution of these isolated deuterium plasma clouds in a uniform magnetic field are compared with computer-generated predictions based on various highly simplified models. Since they are made from solid pellets by one-sided cross-field laser irradiation, these clouds are initially not spherically symmetric. Nevertheless, the crossfield expansion is arrested approximately as expected for complete flux exclusion, and during this early phase the generated dipole moment is not easily distinguishable from that of expanding perfectly conducting axisymmetric shells. Such clouds, however, would overshoot and oscillate about an equilibrium diameter and would also slow down parallel to $B$. Actual clouds $\left(\mathrm{N} \approx 10^{16}\right.$ atoms), on the other hand, keep expanding along the field and show no evidence of multiple "bouncing," possibly because of their flute-1ike distortion or because of anomalous ficld diffusion, or both.

\section{INVESTIGATION OF FLUTE MODES AND OF PLASMA-FIELD INTERPRETATTON}

This phase of the work is incomplete and only preliminary results can be given. It appears that relatively small pellets $\left(\mathrm{N}_{i}<2 \times 10^{15}\right)$ irradiated by wel1 aligned high-intensity (W $>5 \times 10^{11}$ watt $/ \mathrm{cm}^{2}$ ) ruby laser light have a fair chance of yielding clouds expanding along the B-field as tight cylindrical plasma column with only minor or even undetectable flute development, as shown 


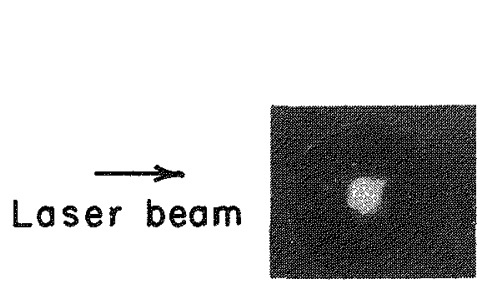

(a)

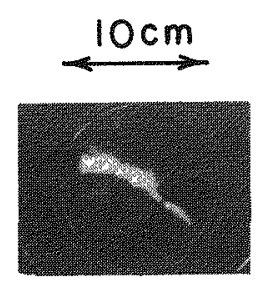

(b)

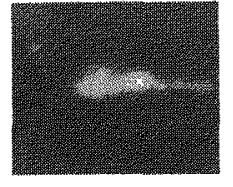

(c)

Fig. 2. Photographs of scintillator images produced by plasma streaming along B-field and striking the plasma camera at a distance of $56 \mathrm{~cm}$ from the "zap-zone". Conditions are:
(a) $B=7.4$ kilogauss, $N_{i} \approx 2 \times 10^{15},\left\langle\varepsilon_{i}\right\rangle \approx 250 \mathrm{eV}$;
(b) $B=7.0$ kilogauss, $N_{i} \approx 2 \times 10^{15},\left\langle\varepsilon_{i}\right\rangle 100 \mathrm{eV}$;
(c) $B=4.0$ kilogauss, $N_{i} \approx 4 \times 10^{15},\left\langle\varepsilon_{i}\right\rangle 100 \mathrm{eV}$.

$(\mathrm{XBB} 751-576)$

in Fig. 2(a). The cross-field diameter of the plasma in this case is only a few ion gyroradii. Large pellets, on the other hand, and particularly those with relatively low energies per particle, invariably display strong flutes, predominantly in the $m=2$ mode, and these flutes tend to be aligned with the direction of the incident laser beam. Examples are shown in Figs. 2(b) and (c). This observation is consistent with the non-symmetric initial expansion of the plasma clouds, mentioned in the preceeding two subsections, which is more pronounced when the "burn time" is not negligible compared to the ideal MHD stopping time in the magnetic field. Moreover, large low-temperature clouds have less chance of flute stabilization by finite-gyroradius effects than small hot ones.

In either case it is found, that the intermixing of the plasma with the magnetic field is faster than expected for classical diffusion. The rapid transition of the plasma to a state of $10 w-\beta$ is not yet fully understood and needs to be investigated more carefully. Further work aimed at shedding more light on these processes is in progress.

\section{References and Footnote}

*Present address: Los Alamos Scientific Laboratory, Los Alamos, New Mexico 87545.

1. Controlled Thermonuclear Research Annual Reports, Lawrence Livermore Laboratory Reports UCRL-50002-69 to UCRL-50002-73 (1969-73).

2. F.H. Coensgen, W.F. Cummins, W.E. Nexsen, Jr., and A.E. Sherman, Rev. Sci. Instrum, (1964).

3. T.R. Jarboe and W.R. Baker, Rev. Sci. Instrum. 45,431 (1974).

4. T.R. Jarboe, Study of an Isolated LaserProduced Deuterium Plasma in a Magnetic Field, (Ph.D. Thesis), Lawrence Berkeley Laboratory Rpt. LBL-3097 (1974).

\title{
ATOMIC PHYSICS SIUDIES
}

\author{
K.H. Berkner, W.G. Graham, D. Leung, T.J. Morgan, R.V. Pyle
} S.E. Savas, P.J. Schneider, and J.W. Steams

\section{PRODUCTION AND DESTRUCTION OF H"}

The atomic physics program of the CTR Group has started three different experimental programs to study the formation and destruction of $\mathrm{H}^{*}$ ions, a topic of considerable interest to the neutral-beam injection program. The past year has been spent in the fabrication of apparatus; it is hoped that al1 three will be operational during 1975 .

Formation of $\mathrm{H}^{-}$by Backscattering from Clean Metal1ic Surfaces

Several years ago we studied electron-capture by 5 - to $150-\mathrm{keV} \mathrm{H}{ }^{+}$beams transmitted through thin carbon foils whose exit surfaces were coated in situ with a fresh layer of metal. I It was observed that at $4 \mathrm{keV}$ about $12 \%$ of a hydrogen beam exiting from a clean $\mathrm{Mg}$ surface was $\mathrm{H}^{-}$; furthermore, the $\mathrm{H}^{-}$yield increased with decreasing energy. Since this was a transmission experiment, the large angular scatter of the beam made it impossible to take measurements at lower energies. It is we 11 known that at energies below several $\mathrm{keV}$ a large fraction of protons incident on a surface are scattered back out of the surface. From our experience with the transmitted-beam experiment, we would expect a large fraction of the backscattered beam to be $\mathrm{H}^{\prime \prime}$. We have, the: fore, modified the apparatus used for the trans mission experiment to study the charge composition of low energy $\mathrm{H}^{+}$beans backscattered from a clean metallic surface. Preliminary experiments with $\mathrm{Mg}$ surfaces should begin early in 1975 . 
Charge Exchange of $\mathrm{H}^{+}$Beams in Metal Vapors

A more usual approach for producing $\mathrm{H}^{-}$ions is two-electron capture in metal vapors by low-energy ( 1-1. keV) protons. One would produce highenergy neutral beams for injection into CTR devices by starting with a low-energy $\mathrm{H}^{+}\left(\mathrm{D}^{+}\right)$beam, converting it to $\mathrm{H}^{-}$in a metal vapor with the highest possible efficiency, accelerating the $\mathrm{H}^{-}$ to several hundred $\mathrm{keV}$, and then stripping the $\mathrm{H}^{-}$ (possibly in a plasma target--see next section) to produce energetic $H^{0}$. For optimum system efficiency it is desirable to find a metal-vapor target which has a large efficiency for producing $\mathrm{H}^{-}$ at $\sim 5$ to $10 \mathrm{keV}$; at lower energies it is difficult to produce and transport a high-current ion beam and at higher energies there would be a considerable amount of wasted power in the $\mathrm{H}^{+}$and $\mathrm{H}^{0}$ fraction of the beam.

The metal-vapor targets that have been studied most extensively are the alkali metals. There is considerable scatter in the available measurements, but none of the alkali metals seem to give $\mathrm{H}^{-}$yields greater than $10 \%$ in the desired energy range.

Hiskes at LLL has made rough theoretical estimates of expected $\mathrm{H}^{-}$yields and has proposed that the alkaline earth metals may give more favorable yields.

A target oven has been fabricated and beamline parts are being assembled for an experiment. to measure the $\mathrm{H}^{-}$yields in alkaline earth targets.

\section{$\mathrm{H}^{-}$Interactions with a Plasma}

We, and others, have measured the relevant cross sections determining the conversion of fast $\mathrm{H}^{-}$and $\mathrm{D}^{-}$ions to $\mathrm{H}^{0}$ and $\mathrm{D}^{0}$ atoms in comnon gases. For deuterons of $150 \mathrm{keV}$, an injection energy considered desirable in some controlled thermonuclear reactor designs, the $\mathrm{H}^{-} \rightarrow \mathrm{H}^{0}$ conversion efficiencies in optimized gas targets are 60-65\%. Sweetman, et al. ${ }^{2}$ estimated that the conversion efficiency might be as high as $90 \%$ in an optimized fullyionized hydrogen plasma target.

Apparatus to produce a dense hydrogen plasma target has been built and is being tested; a pulsed, high-current, hot cathode arc discharge in a magnetic field is being diagnosed with microwave interferometer and Langmuir probes. When a suitable plasma line density (ne $\approx 10^{15}$ ions $/ \mathrm{cm}^{2}$ ) has been obtained, we will measure the conversion of 100 to $150 \mathrm{keV} \mathrm{D}^{-}$ions to neutral atoms.

ELECTRON-TRANSFER AND DISSOCIATION CROSS SECTIONS OF $1.15-$ TO $26-\mathrm{keV} \mathrm{H}, \mathrm{H}^{0}, \mathrm{H}^{-}$, and $\mathrm{H}_{2}^{+}$IN COLLISTONS WITH Xe GAS

We have measured total cross sections of 1.25- to $26-\mathrm{keV} \mathrm{H} \mathrm{H}^{+}, \mathrm{H}^{0}, \mathrm{H}^{-}$, and $\mathrm{H}_{2}^{+}$colliding with the xenon atom. Over the present energy range, there have been several pertinent experimental studies of total cross section measurements in xenon gas; however, there are gaps in the energy ange and some discrepancies in magnitude and in hergy dependence of the cross sections. Some of he pertinent cross sections have not been measured and several of the interactions have been measured only once. Also, different experimental techniques have been used to measure the cross sections. Therefore, we felt it desirable to obtain a self- consistent set of cross sections by measuring the elementary interactions between $\mathrm{H}^{+}, \mathrm{H}^{0}, \mathrm{H}^{-}$, and $\mathrm{H}_{2}^{+}$and the xenon atom using the same experimental technique and apparatus.

We have made measurements of the following processes in xenon gas: ${ }^{3}$

$$
\begin{aligned}
& \sigma_{10}: \mathrm{H}^{+} \rightarrow \mathrm{H}^{0} \\
& \sigma_{11}: \quad \mathrm{H}^{+} \rightarrow \mathrm{H}^{-} \\
& \sigma_{01}: \mathrm{H}^{0} \rightarrow \mathrm{H}^{+} \\
& \sigma_{01}: \quad \mathrm{H}^{0} \rightarrow \mathrm{H}^{-} \\
& \sigma_{\overline{1} 0}: H^{-} \rightarrow H^{0} \\
& \sigma_{\overline{11}}: \mathrm{H}^{-} \rightarrow \mathrm{H}^{+} \\
& \sigma_{+}: \quad \mathrm{H}_{2}^{+} \rightarrow \mathrm{H}^{+} \\
& \sigma_{-}: \mathrm{H}_{2}^{+} \rightarrow \mathrm{H}^{-} \\
& \text {(single electron capture) } \\
& \text { (double electron capture) } \\
& \text { (single electron 1oss) } \\
& \text { (single electron capture) } \\
& \text { (single electron loss) } \\
& \text { (double electron 1oss) } \\
& \text { (proton production) } \\
& \sigma_{n}: \quad H_{2}^{+} \rightarrow H_{2} \\
& \text { (negative ion production) }
\end{aligned}
$$

These measurements have been completed and a report of the work is being prepared. Preliminary results for $\mathrm{H}_{2}^{+}$collisions ${ }^{4}$ are shown in Fig. 1. Unfortunately, these measurements do not corroborate the rumored large $\mathrm{H}_{2}^{+} \rightarrow \mathrm{H}^{-}$conversion efficiencies.

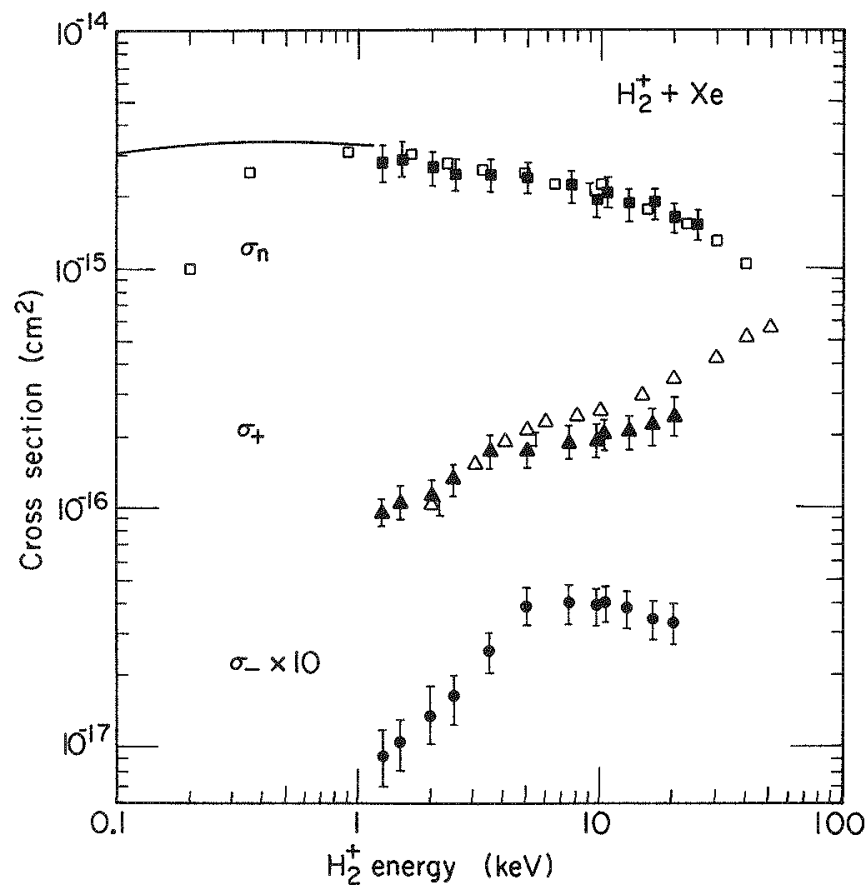

Fig. 1. Cross sections $\sigma_{n^{\prime}} \sigma_{+}$, and $\sigma_{-}$for the formation of $\mathrm{H}_{2}$ and $\mathrm{H},{ }_{\mathrm{H}^{+}}$, and $\mathrm{H}^{-}$for collisions of energetic $\mathrm{H}_{2}^{+}$ions with Xe gas. $\sigma$ : present results; $\square$, Stedeford and ${ }^{\mathrm{n}}$ Hasted (Ref. 1); - Koopman (Ref. 5). $\sigma_{+}:$, present results; $\Delta$, Williams (Ref. 6 ). $\sigma_{-1}: 0$, present results. Note: the cross section $\sigma$. has been multiplied by ten. Absolute uncertainty in the data is $\pm 10 \%$ and the error bars indicate the relative uncertainty.

(XBL 7411-8259) 


\section{Footnote and References}

* Summer 1974 only. Permanent address: Wesleyar University, Middletown, CO.

1. K. H. Berkner, I. Bornstein, R. V. Pyle, and J. W. Stearns, Phys. Rev. 6A, 278 (1972).

2. D. R. Sweetman, et al., in Plasma Physics and Controlled Nuclear Fusion Research 1971 (IAEA, Vienna, 1971), Vol. III, p. 393, Section 2.4
3. The motivation for this research was a report that a European group had observed very large cross sections for converting $\mathrm{H}_{2}^{+}$ions to $\mathrm{H}^{-}$ ions in Xe gas.

4. T. J. Morgan, K. H. Berkner, R. V. Pyle, and J. W. Stearns, Bul1. Am. Phys. Soc. 19, 1175 (1975)

5. D. W. Koopman, Phys. Rev. 15479 (1967).

6. J. F. Williams D.N.F. Dunbar, Phys. Rev. 149 $62(1966)$.

\section{NEUTRAL-BEAM DEVELOPMENT AND TECHNOLOGY*}

W. R. Baker, K. H. Berkner, W. S. Cooper, K. W. Ehlers, W. B. Kunke1, R. V. Pyle, and J.W. Stearns

The objective of the neutral-beam program is to develop the hardware required by experimental confinement devices and to advance the technology for injectors needed for power-producing fusion reactors planned for the $1980^{\prime} \mathrm{s}$ and beyond. The LLL/LBL neutral beam development group is responsible for meeting the needs of the mirror program; devices resulting from this program also wi11 be made available to the Tokamak program. This pulsed-beam work is based on extending the 10 ampere (equivalent neutral beam), $20-\mathrm{keV}$ sources described in a previous CTR annual report 1 to higher currents and energies.

The pulsed-beam development in 1974 mainly was oriented toward the following near-term programmatic goals:

July 1974: $100 \mathrm{~kW}$ of neutral beam into ATC (Adiabatic Toroidal Compressor) at the Princeton Plasma Physics Laboratory.

Sept. 1974: Sources providing $600 \mathrm{~A}$ at $20 \mathrm{keV}$ on the 2XIIB mirror machine at LLL

One $50-\mathrm{A}, 20 \mathrm{keV}$ source for the BBII mirror experiment at LLI

Two $50-\mathrm{A}, 20-\mathrm{keV}$ sources for ATC.

The goal of $100 \mathrm{~kW}$ into ATC was accomplished by redesigning previously built 10-A sources to operate with curved accelerating electrodes that focus the beam in one dimension so that a greater fraction of the total neutral beam passes through the sma11 ATC entrance aperture.

To satisfy the remaining near-term objectives we designed and tested 50-A neutral-beam modules (12 of these 50-A modules are used on 2XIIB). Each of these 20-keV, 10-millisecond pulse length modules can deliver about 1.2 megawatts of neutral beam into $a \pm 2^{\circ} \times \pm 4^{\circ}$ solid angle (a $20 \mathrm{~cm} \times 40 \mathrm{~cm}$ aperture $290 \mathrm{~cm}$ from the source), of which 0.8 megawatts will pass through a $10 \mathrm{~cm} \times 20 \mathrm{~cm}$ aperture at $290 \mathrm{~cm}$.

A photograph of the plasma chamber in which the ions are produced and the $20-\mathrm{kV}$ extractor assembly is shown in Fig. 1. The plasma source is designed to operate with arc currents up to 4,000 A, drawn from 86 tungsten filaments. The three-electrode, $7 \times 35 \mathrm{~cm}$ extraction area, acceleration structure shown in the figure has flat electrodes; later models use the curved focusing structures designed by T. J. Duffy of LLL, mentioned previously in regard to the ATC "10-A" sources. After the ions have been accelerated to $20 \mathrm{keV}$ they pass through a neutralizer cell where about $90 \%$ are converted to neutral atoms and molecules.

The power required for $20 \mathrm{kV}$ deuterium operation of a $50-\mathrm{A}$ source is $25 \mathrm{~kW}$ to the filaments, $160 \mathrm{~kW}$ in the arc, $1.6 \mathrm{MW}$ of acceleration power, and $15 \mathrm{~kW}$ of decel power. The gas flow is 21 Torrliters/second.

To meet the intermediate-term needs, testing of higher voltage sources should begin on the following approximate schedule:
January 1975:

July 1975:

January 1.976:
$50 \mathrm{~A}, 40 \mathrm{keV}, 10 \mathrm{msec}$

$50 \mathrm{~A}, 60-80 \mathrm{keV}, 10 \mathrm{msec}$ $25 \mathrm{~A}, 40-80 \mathrm{keV},>20 \mathrm{msec}$.

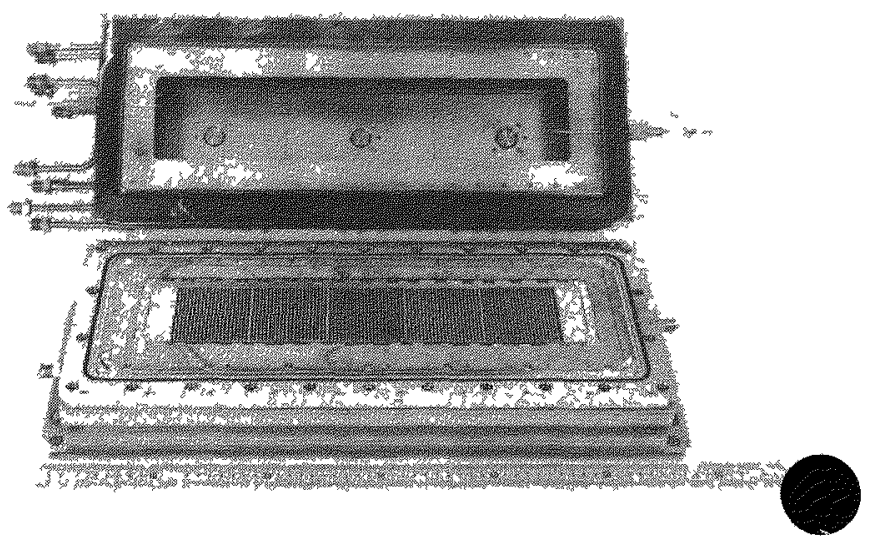

Fig. 1. Photograph of plasma chamber and extractor of the 50-A source. 
One method of raising the energy of beams extracted from present-generation large-area multible-aperture (slots or holes) neutral-beam sources is the staging of accelerator gaps. As the first step in this direction, we have added an additional accelerator electrode to one of the LBL threegrid single-stage extractors, which delivered $10 \mathrm{~A}$ of $20-\mathrm{keV}$ deuterium beam into $\pm 0.6^{\circ}$ by $\pm 2^{\circ}$. Preliminary results for $20-\mathrm{msec}, 34-\mathrm{keV}$ deuterium beams are as follows: with acce1-accel-dece1 gap voltages of $15.5,20.1$, and $-1.6 \mathrm{keV}$ the extracted current was approximately $10 \mathrm{~A}$; of this, $8.1 \mathrm{~A}$ (equivalent current, about $85 \%$ neutrals) was deIivered to a $20-\times 40-\mathrm{cm}$ calorimeter at $3.3 \mathrm{~m}$ $\left( \pm 1.70^{\circ}\right.$ by $\left.\pm 3.5^{\circ}\right)$ and 4.9 A was delivered to the centrai 10 -by- $20 \mathrm{~cm}\left( \pm 0.9^{\circ}\right.$ by $\left.\pm 1.7^{\circ}\right)$ part of the calor $i$ meter.

The program, WOLF, for calculating and optimizing ion extraction with more realistic input data and physics is now operating and is being applied to extractor design. This program is the first step toward a model containing enough physics of the plasma and of the extraction process to predict accurately the performance of a given extractor, and then to optimize the extractor design for a given task.

The program treats symmetric or asymmetric two-dimensional extractors (slots) with no magnetic field. Ion flow with space charge is calculated by solving the equations of motion and Poisson's equation iteratively on a flexible triangular mesh attached to the boundaries. The emitting surface is assumed to be a flexible surface at the position of the plasma sheath edge. Ions are assumed to arrive at this surface with a distribution in directed velocities to simulate an ion wind; a nonzero ion temperature and the effects of electrons in the sheath are included. The magnitude of the electric field $E_{0}$ on the surface must also be specified. The ion velocity distribution and $\mathrm{E}_{0}$ must be calculated, assumed, or derived from measurements of the plasma properties. The ion current density $\mathrm{j}^{+}$can be specified or treated as a variable. WOLF then varies the shape of the emitting surface until the electric field at each mesh interval on the emitter equals $E_{0}$ in a least-squares sense equivalent to specifying $j^{+}=$constant on the surface, and determines the shape that the plasma sheath edge will assume in the vicinity of the extractor electrode. In addition, the program can vary the shape and potential of selected electrodes to minimize the beam divergence.

So far WOLF correctly calculates all measured beam properties if the ion temperature in the source is assumed to be slightly over $1 \mathrm{eV}$, in agreement with the value required to explain the beam divergence parallel to the slots (in this direction there are no electric fields to de-focus the beam).

\section{Footnotes and References}

* This work is part of a program of neutral beam development being conducted jointly by the Lawrence Livermore Laboratory (LLL) and LBL. It has also been reported in the LLI report series UCRL -50002 .

1. Controlled Thermonuclear Research Annual Report, UCRL-50002-73, p. 92 (1973).

\section{TORMAC SHEATH CONFINEMENT}

M. A. Levine, I. G. Brown, and W. B. Kunke1

\section{INTRODUCTION}

In the United States, the study of magnetic high-temperature plasma confinement for controlled thermonuclear fusion has been narrowed down primarily to three major concepts: Tokamak, Scy1lac, and the minimum-B mirror. However, all three are still faced with great difficulties, and it would be a mistake at this time not to pursue altemative ideas. One new idea is the toroidal line cusp system called Tormac.1-3 A thorough review and evaluation of the Tormac concept was carried out by an ad-hoc panel of experts in the field chaired by Marsha11 N. Rosenbluth of the Institute for Advanced Studies at Princeton. The Rosenbluth Panel concluded that the Tormac concept had great potential for controlled fusion, but many serious questions needed to be resolved before a final verdict on the concept could be given. In response to the pane1 recommendation for further work on the Tormac project, an upgraded experimental program, supplemented by improved diagnostics, is eing conducted at LBL.

\section{EXPERIMENTAL WORK}

Two experiments in progress are designed to answer different questions about the Tormac con- cept. The smaller experiment, "Shaker", is concerned with heating in Tormac; whereas a larger and hotter experiment, "Baker", is set to study sheath structure and confinement characteristics.

Both the Shaker and Baker experiments are designed around the toroidal bicusp shown in Fig.1.

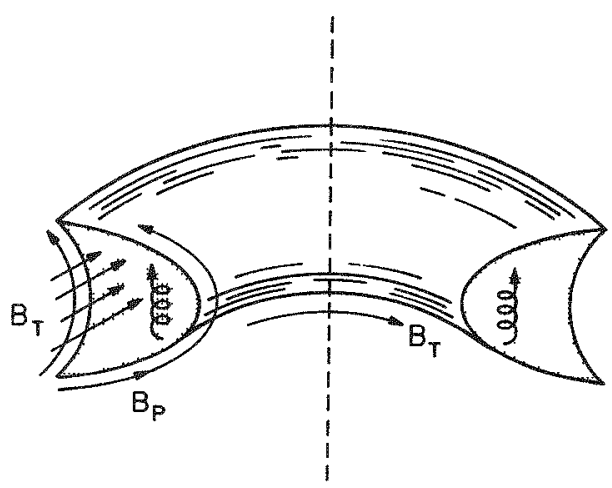

Fig. 1. Tormac plasma (shaded area) contained in a toroidal magnetic bicusp with poloidal, $B_{p}$, and toroida1, $\mathrm{B}_{\mathrm{T}}$, components. The diagram indicates a vertical drift of particles in the absence of a rotational transform.
(XBL 751-2085) 
In the Shaker heating experiment a toroidally symmetric magnetoaoustic wave is launched into the plasma from the outside using a single-turn coil wrapped around the outer circumference of the chamber.

The equilibrium shape of the plasma in the bicusp magnetic field with and without current in the heating coil has been determined from modeling experiments in an electrolytic tank. 4 A $50-\mathrm{cm}$ outside diameter bottle with magnetic field coils and suitable diagnostic ports has been constructed.

For Shaker a computer program has been written to determine the optimum heating rate. The program is predicated on a circuit for the one-turn heating coil which uses two capacitors and two switches for pulse shaping. The plasma characteristics are included using a complete wave absorption model for the plasma. 5

For the Baker experiment a 1-meter glass chamber has been designed with the idea of containing a $300-\mathrm{eV}$ plasma at densities of $10^{15}$ particles/cc. A most important feature of the Baker experiment is that it will include diverter-1ike chambers as shown in cross section in Fig. 2 .

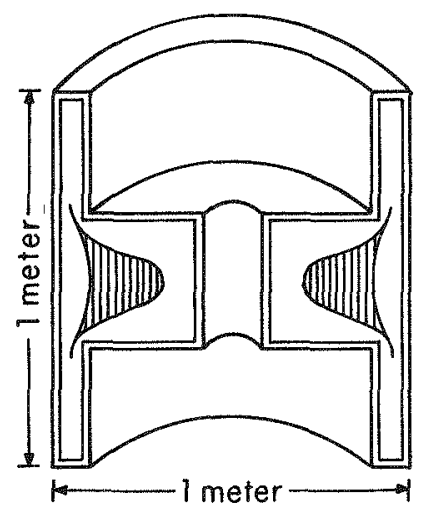

Fig. 2. Glass vacuum vessel with plasma (shaded area). Cylindrical extensions act as diverter chamber for escaping plasma.

(XBL 751-2086)

\section{THEORETICAL WORK}

One serious theoretical question about the Tormac concept concerns the problem of toroidal drift of particles in the central region of the plasma. Because of the magnetic field curvature and magnetic field gradients, particles within the central region of Tormac, as shown in Fig. 1, will tend to drift in a vertical direction. Particle drifts would perform orbits that would close in the outside sheath region near the edge of the plasma. This creates a problem because in the outside sheath region the plasma is contained on open magnetic field lines by magnetic mirroring and thus the plasma in the sheath region has a nonisotropic distribution function which is characteristic of mirror-contained plasma. The problem is that drifting particles will communicate this distribution function to the entire plasma and degrade the containment characteristics of Tormac. A rotational transform that circulates plasma around the minor axis of the torus would eliminate or reduce the particle drifts to a point where they no longer affect the plasma containment. One method of doing this is to set the plasma in rotation about the minor axis.

If the plasma in a Tormac configuration is set into poloidal rotation (around the minor axis) the condition for a stationary state is $B(R) / R n(R)=$ const. ${ }^{6}$ The plasma density $n(R)$ in the interior can thus not be uniform in such cases but must decrease along the major radius $\mathrm{R}$. For slow rotation, the equation of motion can be integrated directly. The solution $n(R)$ is found to be marginally MD-stable, i.e., it satisfies $\mathrm{Rd} n / \mathrm{dR}=-4 \mathrm{n} /(2+\gamma \beta)$ for all values of $\beta$. For large $\beta$ we find $n \approx$ const., i.e., $B \propto R$ so that curvature and grad-B drifts tend to cance1. This condition is interesting for Tormac confinement because then only a modest rotation speed is sufficient to prevent the guiding centers of most particles from drifting to the boundaries. In this way the anisotropy in velocity space caused by losses at the cusps will not be rapidly communicated to the interior. Rotation can be enhanced and controlled in principle by neutral injection with angular momentum about the minor axis.

\section{Footnotes and References}

*Work supported in part by the Electric Power Research Institute.

1. C. C. Ga1lagher, L. S. Combes, and M. A. Levine, Phys. Fluids 13, 1617 (1970).

2. M. A. Levine, A. H. Boozer, G. Kalman, and P. Bakshi, Phys. Rev. Lett. 28, 1323 (1972).

3. A. H. Boozer and M. A. Levine, Phys. Rev. Lett. 31, 1287 (1973)

4. L. S. Combes, C. C. Gailagher, and M. A. Levine, Rev. Sci. Instrum. 11.1567 (1966).

5. A. H. Boozer, to be published (1975).

6. W. B. Kunkel and M. A. Levine, LBL-3231, Dec. 1974. 


\title{
PARAMETRIC INSTABILITIES IN TURBULENT, INHOMOGENEOUS PLASMA
}

\author{
D. R. Nicholson and A. N. Kaufman
}

It is known that a one-dimensional linear parametric instability, with opposed velocities for the undamped decay waves, is "absolutely" unstable for a uniform medium, 1 but saturates for a medium with constant density gradient. 2 In order to provide physical understanding for this apparent paradox, we proposed to study the effect of slight density turbulence (time-independent) superimposed. on the gradient. We argued that the turbulence would destroy the delicate destructive interference which saturates the instability. Extensive numerical solutions of the differential equations representing the wave coupling do indeed show that, above a threshold turbulence level, the saturation disappears and is replaced by absolute instability at a rate dependent on the rms value and correlation length of the turbulence. The threshold level is much lower than predicted by previous work, 3 based on a more specialized model. We concluded that one instability (that producing the turbulence) may drastically modify the development of another instability.

\section{Footnote and References}

* Condensed from Phys. Rev. Lett. 33, 1207 (1974); LBL-3302.

1. D. Bobroff and H. Haus, J. App1. Phys, 38, 390 (1967).

2. M. Rosenbluth, Phys. Rev. Lett. 29, 565 (1967).

3. P. Kaw, R. White, D. Pesme, M. Rosenbluth, G. Lava1, R. Varma, and R. Huff, Trieste Report IC/73/142 (1974).

\section{ACTION CONSERVATION IN THE PRESENCE OF A HIGH-FREQUENCY FIELD*}

\author{
A. N. Kaufman and L. Stenf10 ${ }^{\dagger}$
}

The laws of action conservation, also known as the Manley-Rowe relations, occupy a peculiar place in plasma theory and application. Some people 1 consider them "obvious"; others ${ }^{2}$ feel constrained to verify their validity after a specific calculation, sometimes to check the calculation; others 3 attempt to develop a general proof; yet others 4 search for ways to violate them. Frequently they are overlooked, leading to invalid conclusions. 5 Their importance in applications is that they may serve as a constraint to impede the coupling of radiant energy to plasma heat; 6 also they greatly affect the character of asymptotic states. 5

We feel the subject is still open, and any new evidence is welcome. We have made a test of the laws of action conservation based on the effects of a strong pump field, which greatly modifies the normal modes and mode-coupling coefficients. 7 A straightforward calculation leads to affirmation of their validity here. We feel that a general proof of validity is still urgently needed.

\section{Footnotes and References}

*Condensed from LBL-3309, Plasma Phys. 17, 403 (1975).

†Umeå University, Sweden.

1. E.G., V. Tsytovich, Nonlinear Effects in Plasma (Plenum, N.Y., 1970).

2. E.g., R. Sagdeev and A. Galeev, Nonlinear Plasma Theory (Benjamin, N. Y., 1969).

3. E.g., L. Altshul and V. Karpman, Sov. Phys. JETP 20, 1943 (1965).

4. E.g., K. Mima, J. Phys. Soc. Japan 34, 1620 (1973)

5. E.g., the "law" of equipartition of energy is invalidated by action conservation. For a discussion, see R. Davidson and A. Kaufman, J. Plasma Phys. 3 , 97 (1969).

6. B. Cohen, A. Kaufman, and K. Watson, Phys. Rev. Lett. 29, 581 (1974).

7. L. Stenflo, Plasma Phys. 16, 577 (1974).

\section{NONLINEAR PENETRATION OF A LANGMUIR WAVE INTO A PLASMA DENSITY GRADIENT*}

\author{
A. Kaufman
}

It has become widely recognized that an electron plasma wave can trap itself by producing a propagating depression in plasma density. Such an entity, much studied in a uniform medium, is

lled a "soliton". 1 We expected that a density adient would have an important effect on its behavior. Indeed we found that in a gradient the soliton no longer propagates, but sits at the "critical surface" where the plasma frequency equals the wave frequency. This entity, now called a "caviton," has been experimentally observed. 2

\section{Footnote and References}

*Condensed from LBL-3098, Phys. Scr. 11, 309 (1975).

1. A. Scott, F. Chu, and D. McLaughlin, Proc. IEEE 61, 1443 (1973).

2. H. Kim, R. Stenzel, and A. Wong, Phys. Rev. Lett. 33, 886 (1974). 


\title{
UPPER-HYBRID SOLUTONS *
}

A. Kaufman and L. Stenflo ${ }^{\dagger}$

Because of the great interest in solutons, which are self-trapped pulses of radiation, we have studied the effect of an ambient magnetic field on the phenomenon. We found a parameter range where the radiation (upper-hybrid) has negative dispersion; this has the consequence that the soliton must propagate supersonically, in contrast to subsonic propagation for positive dispersion, the previously studied case. ${ }^{2}$

\section{Footnotes and References}

*Condensed from LBL-3315, Phys. Scr. 11, 269 (1975).

tUmeå University, Sweden.

1. A. Scott, F. Chu, and D. Mclaughlin, Proc. IEEE 61,1443 (1973).

2. V. Karpman, Plasma Physics 13, 477 (1971).

\section{SIMULATION OF LASER BEAT HEATING OF A PLASMA*}

\author{
B. Cohen, M. Mostrom, D. Nicholson, C. Max, ${ }^{\dagger}$ A. Kaufman, and A. B. Langdon'
}

We have made computer simulations of laserplasma interactions using a new code to perform relativistic particle simulations with fully electromagnetic interaction. Most algorithms for solution of Maxwe11's equations require solving a current-driven wave equation for the vector potential. I In our code, we solve for the electromagnetic fields explicitly by integrating Maxwell's equations along their characteristics. Dawson and Langdon ${ }^{2}$ first used this method in 1966. Gridpoints in the space-time mesh are linked by the vacuum characteristics. Spurious numerical dispersion is minimized, because we solve explicitly for the electromagnetic fields and introduce some smoothing in calculating the transverse current. We have streamlined the code to make it fairly inexpensive to use.

The application we have studied is the heating of plasma by two lasers (of frequencies $\omega_{0}, \omega_{1}$ ) whose beat frequency $\left(\Omega \equiv \omega_{0}-\omega_{1}\right)$ is near the plasma frequency. The nonlinear interaction may be considered as an induced decay $\left(\omega_{0} \rightarrow \omega_{1}+\Omega\right)$, in which the fraction $R$ of the incident power at $\omega_{0}$ is converted to $\omega_{1}$ and $\Omega$, with the fraction $\mathrm{R} \Omega / \omega_{0}$ appearing as a longitudinal plasma oscillation and, because of damping, ultimately as heat. For a linear density gradient, theory ${ }^{3}$ predicts that the efficiency parameter $\mathrm{R}$ depends 1 inearly on the product of the scale length and the input laser intensity, and not at all on the details of the damping except for its sign. The dependence on the ratio of input powers is shown in Fig. 2 of Ref. (3). Further detajls of the theory have been presented in Refs. (4) and (5).

Our most recent work extends the general theory of the interaction, with specific attention to the regime of linear longitudinal response of the nonuniform plasma. This theory was tested by simulation, with excellent agreement obtained.

It should be kept in mind that the process we have studied, involving three electron waves (two transverse and one longitudinal), with no ambient magnetic field, is illustrative of the more general three-wave process, possibly involving ions, and in a magnetic field. Thus, the principle of electron heating, by the damping of a resonant excita tion from the beat of two high-frequency waves, can be extended to the analogous heating of ions in a magnetically confined plasma.

\section{Footnotes and References}

*Condensed from LBL-3023, Phys. Fluids 18, 470 (1975).

'Present address: Lawrence Livermore Laboratory.

1. I. Haber, C. E. Wagner, J. Boris, and J. Dawson, in Proceedings of Fourth Conference on Numerical Simulation of Plasmas (Government Printing Office, Washington, D.C., 1973), p. $126 ;$ R. L. Morse and C. Nielsen, Phys. Fluids 14, 830 (1971); A. B. Langdon, B. F. Lasinski, and W. L. Kruer, in Proceedings of the Sixth Conference on Numerical Simulation of Plasmas, Conference Report 73-0710 (Lawrence Berkeley Laboratory, Berkeley, California, 1973), p. 56.

2. A. B. Langdon, Ph.D. Thesis, Princeton University (1969).

3. A. Kaufman and B. Cohen, Phys. Rev. Lett. 30, 1306 (1973) .

4. B. Cohen, A. Kaufman, and K. Watson, Phys. Rev. Lett. 29, 581 (1972).

5. B. Cohen, Phys. Fluids 17, 496 (1974). 
We have undertaken a systematic study of the equations describing the stimulated near $-90^{\circ}$ scatter of an intense electromagnetic pump wave by electron plasma waves in an inhomogeneous plasma. The beat disturbance between the incident and scattered electromagnetic waves acts as a driver for the local electron plasma wave but with a spatially varying phase-mismatch due to the inhomogeneity in magnetic field strength and/or plasma density. This creates an effective resonance zone for the instability and generally leads to saturation when the scattered wave propagates out of this region. It is of interest then to consider the special case of a sidescattered wave traveling nearly along a plane of constant density and magnetic field strength and therefore essentially remaining within the resonance zone. Our central effort has been to determine analytically the spatial properties and frequency spectrum of the sidescattered waves including the effects of diffraction and refraction of the electromagnetic waves, the damping of the electron plasma waves, and the stabilizing influence of the finite diameter of the pump wave.

The problem can be reduced to that of solving a one-dimensional. Helmholtz or Schrödinger equation with a complex potential containing two zeros and one pole (assuming a linear profile of magnetic field strength and/or plasma density). Numerically calculating and plotting the Stokes and antiStokes lines for this potential and then applying phase-integral or WKBJ techniques, we searched for those configurations of the transition points which would lead to eigenvalues. While verifying the temporal growth rates found by Liu, et a1., we did find an additional set of eigenvalues which remain to be analyzed. However, as the scale length of the inhomogeneity increases to infinity, none of the eigenvalues found approaches a limit equal to the homogeneous growth rate. It is believed that this discontinuity is the result of assuming an infinite plasma in the direction of the density gradient and an infinitely wide plasma and pump wave in the sidescatter direction.

Our formalism allows us to view the problem also as a half-space model (inhomogeneity parallel to the boundary sheet, sidescatter direction perpendicular to boundary sheet) in which case the above eigenvalues are interpreted as spatial growth rates of sidescattered waves driven on the boundary. We plan to investigate the spatial properties of these waves as a function of the inhomogeneity scale length and the frequency spectrum on the boundary. Attention will also be devoted to possible generalizations in order to include other modes in a magnetized plasma. The results could be important for rf heating of a magnetically confined plasma and also for laserpellet fusion schemes.

\section{References}

1. C. S. Liu, M. N. Rosenbluth, and R. B. White, Phys. Fluids 17, 1211 (1974).

\title{
OSCILLATION-CENTER APPROACH TO THE CLASSICAL THEORY OF INDUCED SCATTERING IN PLASMA*
}

\author{
Shayne Johnston
}

Induced scattering is a nonlinear physical phenomenon. It is inherently nonlinear in the sense that it represents a resonant interaction between charged particles and two waves. The process is also potentially nonlinear in a strong-interaction sense; if the two waves are sufficiently coherent, particles can be trapped in the beat potential produced by their mixing. Such beat

trapping lies outside the domain of the conventional weak-turbulence theory of induced scattering in plasma. In our work a canonical formulation of the theory has been developed that is applicable to this extended domain.

Our reformulation is predicated on the notion of a time-averaged beat potential. We have reduced the problem of induced scattering of primary waves to that of resonant interaction with virtual beat waves. The operative principle is that the eduction of two primary waves to one effective wave permits the invocation of single-wave nonlinear methods. The formulation is based upon a canonical transformation to "oscillation-center variables" due to Dewar. 1 This transformation represents a nonsecular two-time-scale modification of Hamilton-Jacobi perturbation theory, and replaces the plasma charges by fictitious "quasiparticles" which follow the time-averaged trajectories of the real particles. The principal thrust of Dewar's paper is a reconciliation of the classical and quantum approaches to quasilinear theory. Essentially, we have carried his transformation forward to second order.

The replacement of a bare particle with a dressed quasiparticle by choosing a representation which eliminates an interaction Hamiltonian is a familiar approach to realizations of the manybody problem. The conventional quasiparticle modes in a plasma consist of bare charges that are dielectrically screened by comoving Debye polarization clouds. More than twenty years ago, Bohm and Pines ${ }^{2}$ pursued this effective dressing one step further. They devised a canonical transformation that eliminated the first-order interaction between the plasma particles and the perturbing wave fields. This transformation, restricted to the classical dymamics of a single plasma charge, was resurrected by Dewar, whose two-time-scale modification provides a formulation appropriate 
for coping with resonant wave-particle interactions. A two-time-scale perturbative approach to the induced scattering of two monochromatic waves is not a new idea. Ott and Dum, 3 and Litvak and Trakhtengerts 4 have considered coherent longitudinal and electromagnetic modes respectively in a nonrelativistic plasma. Our contribution is a unification and extension of the work of these authors, employing more elegant canonical methods.

The oscillation-center approach affords, in particular, a simpler method for deriving the linear induced-scattering matrix elements of weak-turbulence theory, and is of particular value in a magnetoactive plasma where nonlinear formulae are generally complicated and difficult to interpret. We have used this method to rederive in a clear and compact way the matrix element for nonlinear Landau damping in a uniform magnetic field of arbitrary (classical) strength, agreeing with the more lengthy calculations of Porkolab and Chang. 5 We have also considered the induced scattering of two coherent electromagnetic waves in an arbitrarily relativistic plasma, obtaining a linear matrix element more general than any calculated previously. It reduces in the unshielded $^{6}$ or nonrelativistic ${ }^{7}$ imits to known results. The nonlinear extension of our result to the regime of beat trapping has been demonstrated. We have constructed equations which reduce, in the nonrelativistic limit, to those presented recently without derivation by Litvak et a1.8 Finally, we have assessed the potential value of dressed-particle transformations in the more general context of plasma kinetic theory, speculating in particular concerning a reformulation of strong turbulence theory.

\section{Footnotes and References}

* A major portion of this work was performed at Princeton University and supported by the Air Force Office of Scientific Research under contract F44620-70-C-0033. This report is condensed from a paper to appear as a Princeton Plasma Physics Laboratory Report, and to be submitted to Physics of Fluids.

1. R. L. Dewar, Phys. Fluids 16, 1102 (1973),

2. D. Bohm and D. Pines, Phys. Rev. 92, 609 (1953).

3. E. Ott and C. T. Dum, Phys. Fluids 14, 959 (1971).

4. A. G. Litvak and V. Yu. Trakhtengerts, Zh. Eksp. Teor. Fiz. 60, 1702 (1971) [Sov. Phys. JETP 33, 921 (1971).

5. M. Porkolab and R. P. H. Chang, Phys. Fluids 15,283 (1972).

6. $\bar{D}$. B. Melrose, Astrophys. Space Sci. 10, 186 (1971) .

7. V. N. Tsytovich, Nonlinear Effects in Plasma, (Plenum Press, New York, 1970), p. 219.

8. A. G. Litvak, V. I. Petrukhina, and V. Yu. Trakhtengerts, ZhETF Pis. Red. 18, 190 (1973) [JETP Lett. 18, 111 (1973)]. 